\title{
Development of Smart Materials Using Aptamer Based Bionanotechnologies
}

\author{
by \\ Yasir Sultan \\ A thesis submitted to the Faculty of Graduate and Postdoctoral Affairs in partial \\ fulfillment of the requirements for the Degree of \\ Doctor of Philosophy \\ In \\ Chemistry \\ Carleton University \\ Ottawa, Ontario
}

(C)2011

Yasir Sultan 
Library and Archives

Canada

Published Heritage

Branch

395 Wellington Street

Ottawa ON K1A ON4

Canada
Bibliothèque et

Archives Canada

Direction du

Patrimoine de l'édition

395 , rue Wellington

Ottawa ON K1A ON4

Canada
Your file Votre référence

ISBN: 978-0-494-89316-6

Our file Notre référence

ISBN: 978-0-494-89316-6

\section{NOTICE:}

The author has granted a nonexclusive license allowing Library and Archives Canada to reproduce, publish, archive, preserve, conserve, communicate to the public by telecommunication or on the Internet, loan, distrbute and sell theses worldwide, for commercial or noncommercial purposes, in microform, paper, electronic and/or any other formats.

The author retains copyright ownership and moral rights in this thesis. Neither the thesis nor substantial extracts from it may be printed or otherwise reproduced without the author's permission.
AVIS:

L'auteur a accordé une licence non exclusive permettant à la Bibliothèque et Archives Canada de reproduire, publier, archiver, sauvegarder, conserver, transmettre au public par télécommunication ou par l'Internet, prêter, distribuer et vendre des thèses partout dans le monde, à des fins commerciales ou autres, sur support microforme, papier, électronique et/ou autres formats.

L'auteur conserve la propriété du droit d'auteur et des droits moraux qui protege cette thèse. $\mathrm{Ni}$ la thèse ni des extraits substantiels de celle-ci ne doivent être imprimés ou autrement reproduits sans son autorisation.
In compliance with the Canadian Privacy Act some supporting forms may have been removed from this thesis.

While these forms may be included in the document page count, their removal does not represent any loss of content from the thesis.
Conformément à la loi canadienne sur la protection de la vie privée, quelques formulaires secondaires ont été enlevés de cette thèse.

Bien que ces formulaires aient inclus dans la pagination, il n'y aura aucun contenu manquant. 


\section{$\underline{\text { Abstract }}$}

This thesis reports on the research towards the development of a 'smart' fertilizer designed to increase nitrogen uptake efficiency of crops. We aim to utilize bionanotechnologies to develop this fertilizer to take advantage of the (1) nanoscale dimensions found in plant biology and (2) unique properties of nanoscale structures. Our smart fertilizer will consist of an embedded aptamer in a polyelectrolyte coating around the nutrient. For the biosensor component, we utilize aptamers due to their superior binding affinities compared to other natural sensors (such as antibodies) and the fact that they will biodegrade in the environment (e.g., biotic and abiotic degradation). To protect our biosensor and fertilizer we have used polyelectrolyte multilayers as permeable coatings in which an embedded aptamer can be protected from the outside environment, while still being able to bind to its target. In this situation, the target analyte, in our case root exudates implicated in crop growth will bind to the aptamer in the soil which will in turn release nutrients for crop uptake through membrane deformities in the film structure.

Towards this goal, our findings suggest the following:

(1) Aptamers retain their binding affinity when embedded in polyelectrolyte bilayer films with binding affinities comparable with those in solution, suggesting that aptamers may be used as biosensors in polymer matrices (Chapter 2);

(2) Aptamers retain their binding affinity when embedded in polyelectrolyte microcapsules and the rate of diffusion of the model target molecule through 
the walls of these capsules, in our case sulforhodamine B, can be changed upon aptamer-target binding (Chapter 3);

(3) Diffusion of small molecules, other than the aptamer's target, can also be changed upon aptamer-target binding in aptamer embedded polyelectrolyte films (Chapter 4);

(4) Using a possible candidate root exudate L-lysine, we have shown that our model system works in changing the permeability of the polyelectrolyte film, in this case leading to increased diffusion, upon aptamer-target binding possibly allowing for in situ testing (Chapter 5); and

(5) By using aptamer super-assemblies, i.e., nano-aggregates, we can tailor the binding affinities of the same aptamer on demand (Chapter 6 ) in case we need to optimize the binding characteristics of our fertilizer for in situ studies. 


\section{Acknowledgements}

I want to first and foremost thank my supervisor Prof. Maria DeRosa for trusting me in the lab alone in the evenings. She always gave me hope when experiments failed and taught me to never give up. She is the best supervisor a student can ever wish for and I would not trade her lab for any place in the World.

I want to thank Dr.'s Carlos Monreal and Morris Schnitzer at Agriculture and Agri-Food Canada in developing this project, financial support for conferences and letting me use their equipment. You both have had a very big impact in my life and I hope we continue to work together for many years to come. I also want to Thank AnnFook Yang and Denise Chabot at AAFC for help with the Laser Confocal Microscope.

I thank God everyday for giving me such a loving family. My Parents have been with me throughout and no words can express how thankful I am to them. They have made life-changing sacrifices for me and my siblings without thinking twice. My siblings, Sameer (Popo), Maliha and Sana have always looked out for me and kept me out of trouble ever since I was in elementary school. A day doesn't pass without thoughts of my Grandmother who left us 5 years ago. I miss you a lot. My nieces and nephews, Hana, Maham, Azlan and Ayaan have been my refuge from the lab over these past 4 years. I love you all.

Thanks to the DeRosa lab, especially Ryan Walsh for late night discussions on various elements of science and for teaching me the various biological techniques in the lab. Thanks to the Chemistry department of Carleton, including Prof's Crutchley, lanoul, 
and Miller. Prof. Crutchley introduced me to Chemistry in my first year of undergraduate studies and has been a mentor to me ever since. I will never understand why he let me work for him, but I am eternally grateful for his trust.

Thanks to my colleagues and supervisor(s) at Environment Canada who have encouraged me to pursue my degree all these years. A very special thanks to Andy Atkinson, who always pushed me to continue my studies and taught me that a scientist's path is poised with a lifetime of learning. Thanks to my manager Greg Carreau for believing in me and letting me continue my studies and being so supportive. I want to thank Dr. Thomas Davis, a friend, colleague and mentor over these past four years. He guided me in preparation of my exams and thesis along with passing important life lessons about being a graduate student.

Thanks to the undergraduate students I have supervised, especially Johny Abboud for continuing to stick with me even though I can be unbelievably difficult and erratic. Thanks to my friends, old and new, who have taught me important life lessons.

For anyone who picks up and decides to read this thesis, remember that hard work always always pays off and seek the guidance of your professors as they are always full of encouragement and hope in times of despair.

Mama, Papa this is all for you, Thank You! 


\section{Table of Contents}

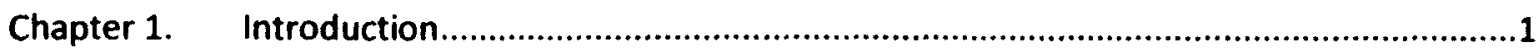



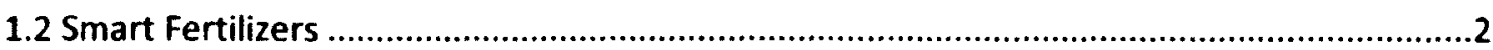

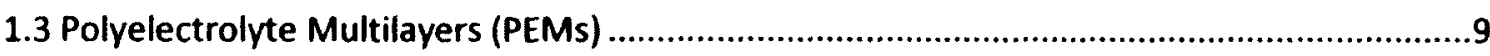

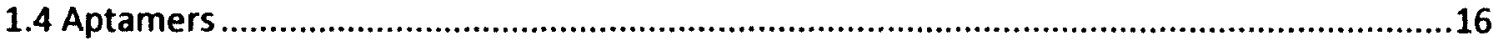

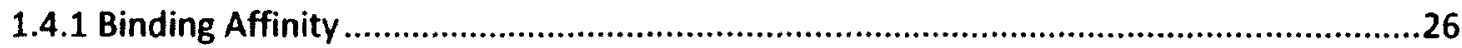

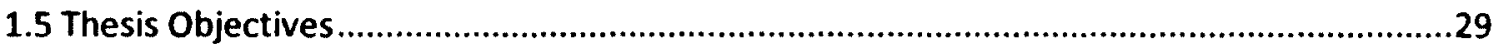

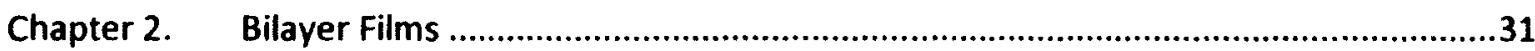

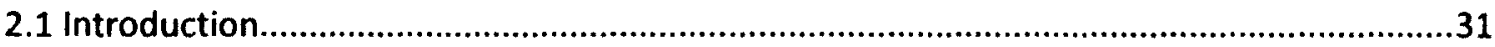





2.1.3 Time-of-Flight Secondary Ion Mass Spectrometry (ToF-SIMS) ....................................36

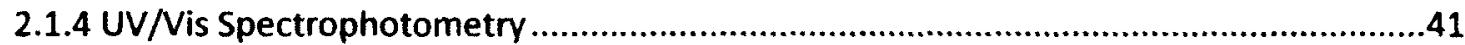

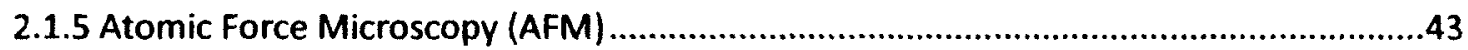

2.1.6 Laser Scanning Confocal Microscopy (LSCM or CLSM)..............................................44

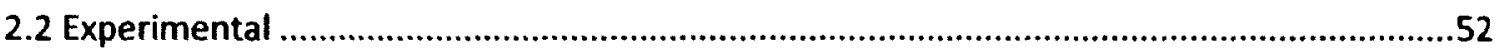

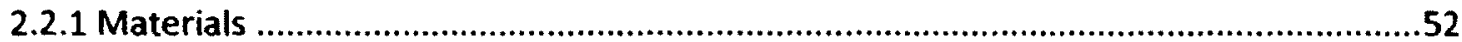



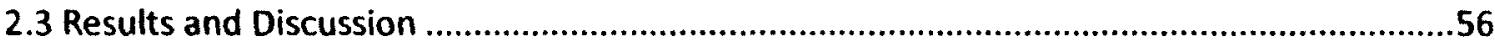

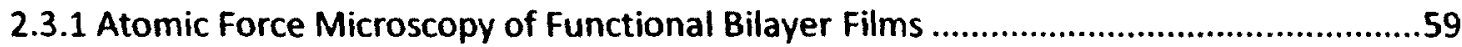

2.3.2 UV/Vis Spectrophotometry of Aptamer Functional Bilayer Films [qualitative analysis]

2.3.3 Confocal Microscopy of Functional Bilayer Aptamer Films [semi-quantitative analysis] .65

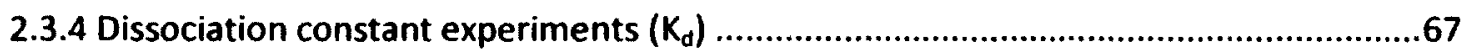

2.3.5 Time of Flight Secondary Ion Mass Spectrometry (ToF-SIMS) [quantitative analysis] 70

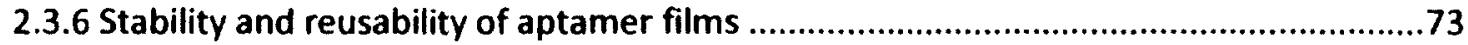

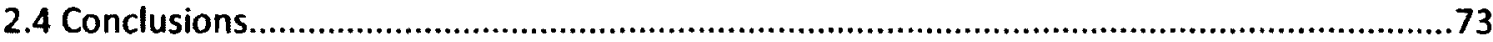



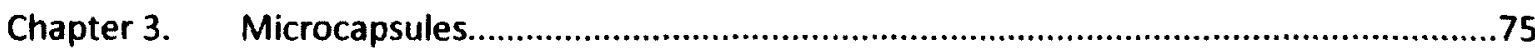




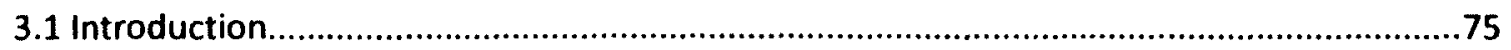

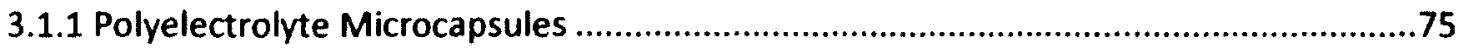

3.1.2 Fluorescence Recovery After Photobleaching (FRAP) ..............................................77

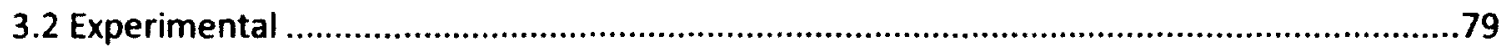

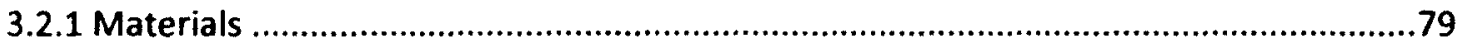

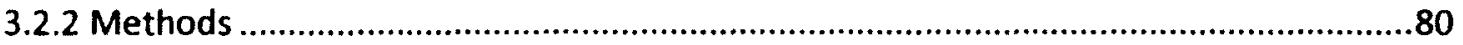

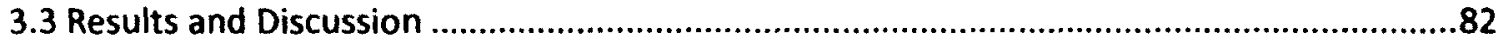

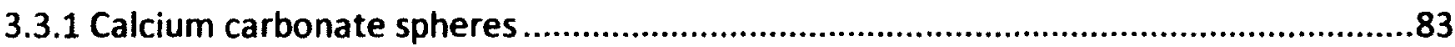

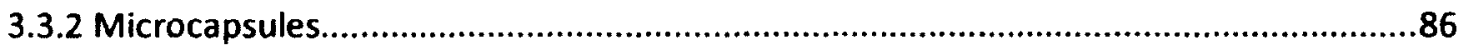

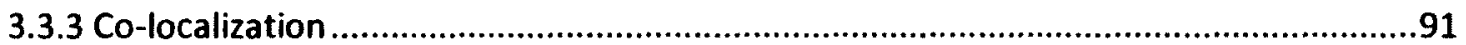

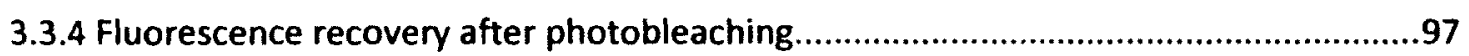

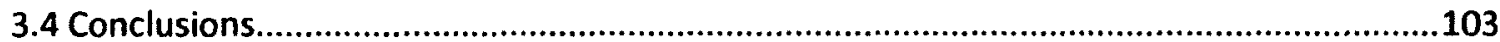

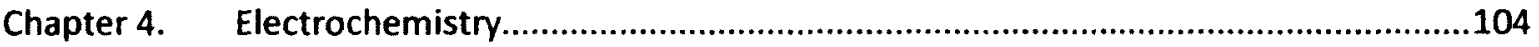

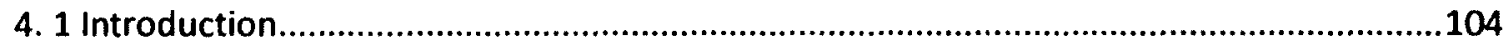

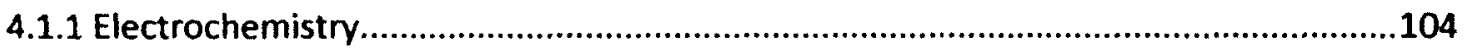

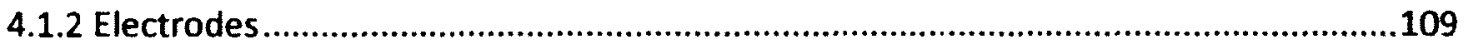

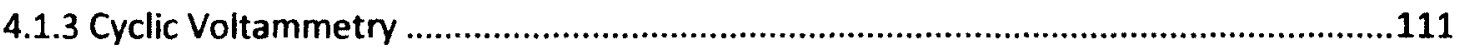

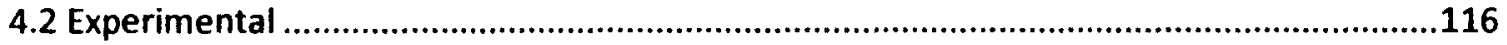

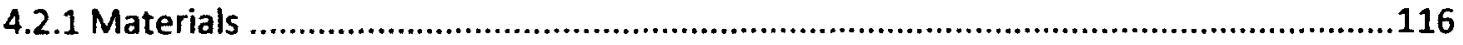

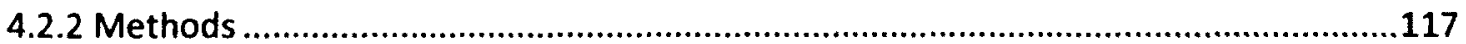

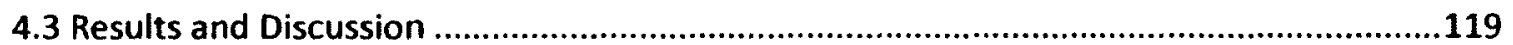

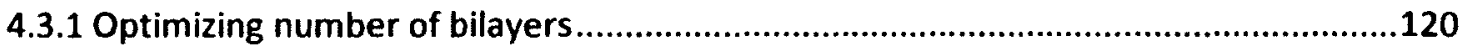

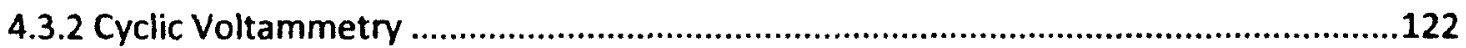

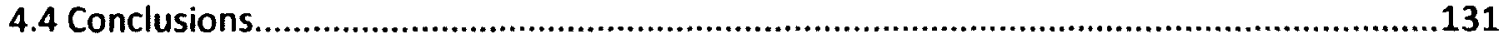

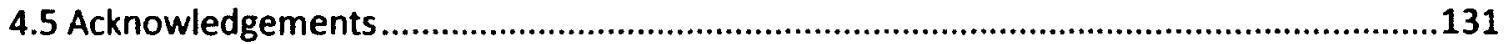

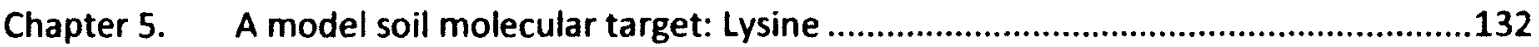

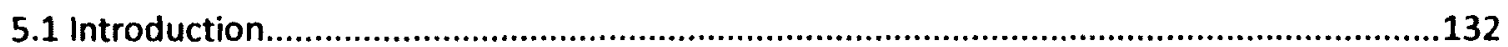

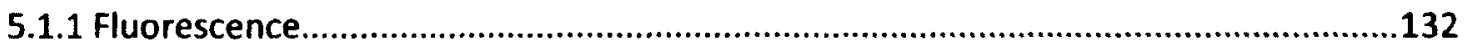

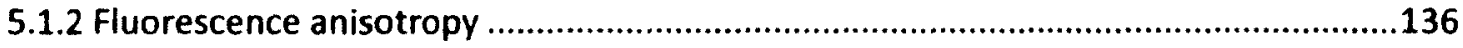

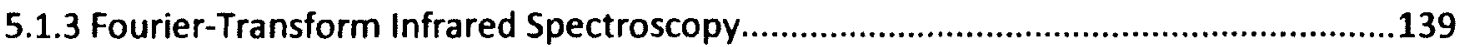

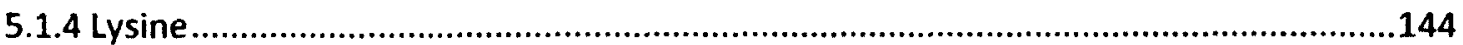




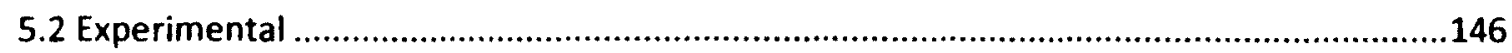

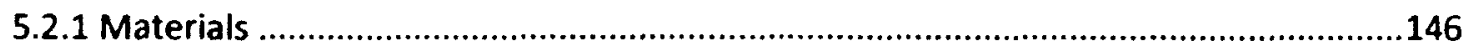

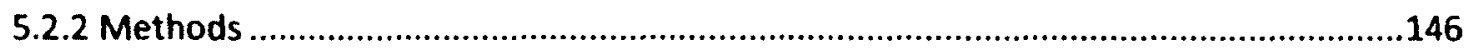

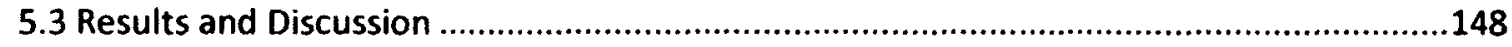

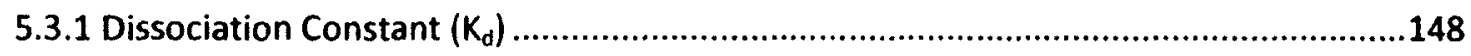

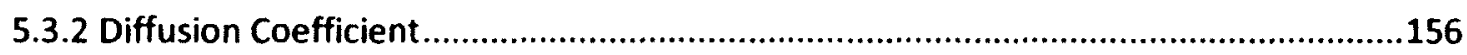

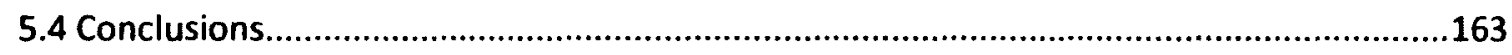

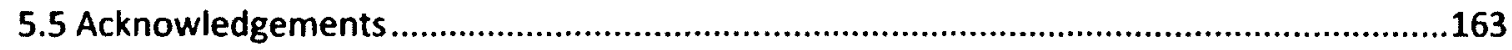

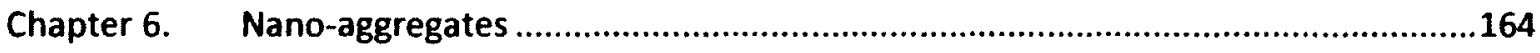

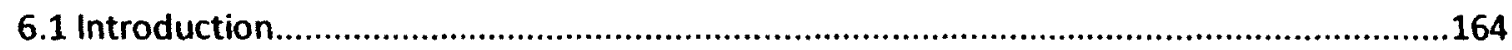

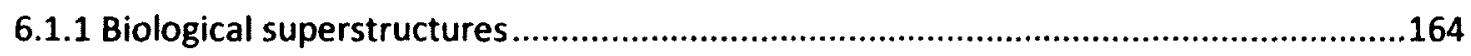

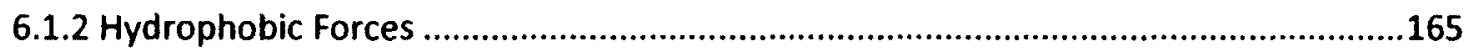

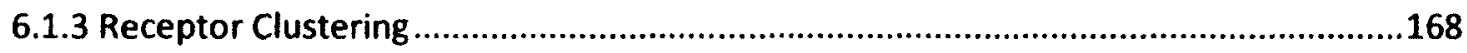

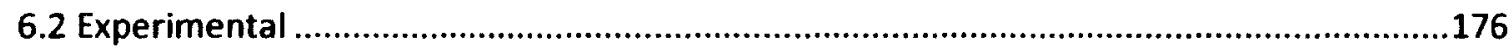

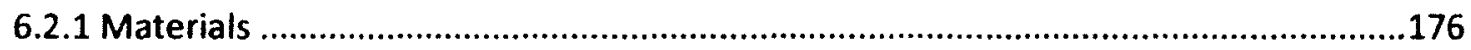

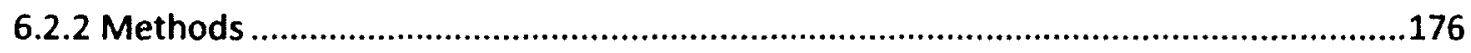

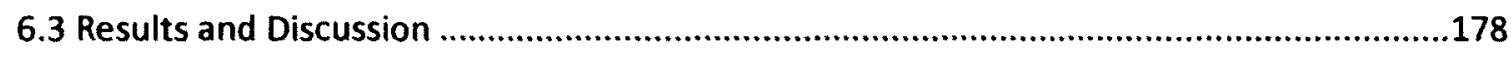

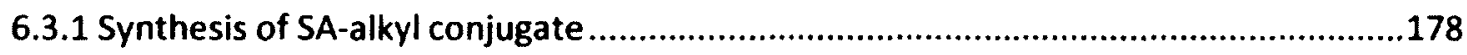

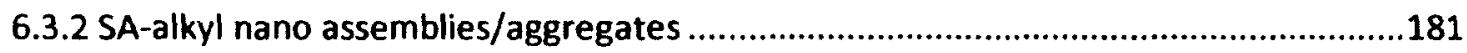

6.3.3 Size selective apparent dissociation constants $\left(K_{d}\right)$...............................................186

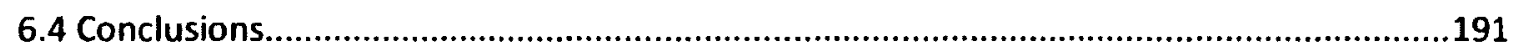

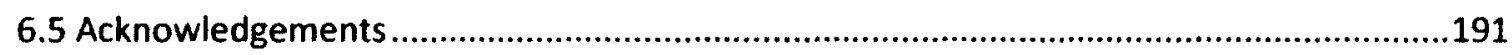

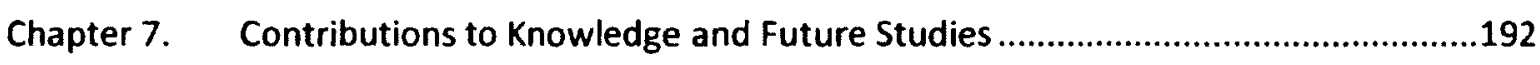

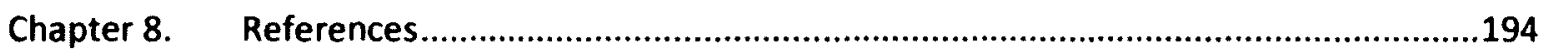




\section{Table of Figures}

Figure 1.1: Nanomaterials can enter plants. (a) improved growth of 27 day old tomato seedling growth in a medium containing carbon nanotubes. (b) Transmission electron micrograph of ryegrass roots showing the entry of zinc oxide nanoparticles (ni, nucleus; ne, nuclear envelope; $\mathrm{np}$, nanoparticles).

Figure 1.2: Possible positive and negative interactions of exudates with soils ${ }^{23}$ .8

Figure 1.3: Lbl self-assembly. A charged substrate, e..g, quartz is dipped into a solution of oppositely charged polyelectrolyte (black string). After saturation of a monolayer, the film is rinsed copiously with water to remove unbound polyelectrolyte. The film is then dipped in oppositely charged polyelectrolyte to absorb a second layer (grey balls, for clarity). This process can be repeated $n$ times. Charge reversal or overcompensation allows for subsequent films to be deposited. For a two polyelectrolyte system, commonly used nomenclature for lbl films is $(A)_{m}(B)_{n}$, where $A$ and $B$ are the polyelectrolytes, and $m$ and $n$ are their respective layers.

Figure 1.4: Intrinsic and Extrinsic charge compensation, where a 1:1 stoichiometric compensation of charge by only the polyelectrolytes results in an intrinsic compensation and if salt ions partake in the compensation, extrinsic charge compensation occurs.

Figure 1.5: Charge overcompensation showing intrinsic compensation (1:1) stoichiometry; (b) introduction of extrinsic charge compensation which causes the film to swell as it allows to decrease steric repulsion still in 1:1 stoichiometry (no charge overcompensation); and (c) in the presence of slightly higher amount of one polyelectrolyte (in this case negative), charge overcompensation can be seen on the surface of the negative polymer overcompensated by the salt ions ${ }^{38}$

Figure 1.6: Aptamer forming secondary structures upon binding to its target, cyanocobalamin. The secondary structures in this situation include a three-stranded zipper and a pair of threenucleotide loops.

Figure 1.7: Overview of Systematic Evolution of Ligands by EXponential enrichment (SELEX). A random pool (library) of ssDNA or RNA strands are selected against a target, typically bound to a column or through high throughput methodologies. Unbound DNA/RNA molecules are discarded while bound molecules are collected, typically through competitive elution. The potential candidates are then enriched through PCR. The cycle is then repeated $n$ times, typically with increasing stringency each round. The final strands are then cloned and sequenced, and commonalities amongst the strands sequences can give an indication on the secondary 
structure formed through computational modeling (e.g., q-quadruplex). Binding affinity studies are done on the strands to indicate the best binding 'aptamer'.

Figure 1.8: Types of aptamer biosensors: (a) generic scheme showing detectable signal production upon binding of a molecular recognition element (MRE; e.g., aptamer) and analyte (e.g., target).; (b) an aptamer in its native form is quenched due to fluorophore-quencher pair spatial arrangement, while upon binding to the aptamer the pair is spatially far from each other and quenching cannot occur and a resultant signal is seen; $(c)$ an aptamer in its unbound form is complexed with its antisense counterpart which contains a quencher to quench the fluorophore on the aptamer. Upon target presence, the antisense strand is removed due to increased complimentarity and as the quencher leaves, fluorescence is re-established and can be detected; (d) modifying an aptamer on both ends with a species able to form excimers (such as pyrene), upon target binding the close spatial arrangement allows for excimer formation and subsequent specific excimer fluorescence at a longer wavelength; (e) mass-based sensor showing the change in mass (frequency of an acoustic wave) upon mass addition of the target (used in quartz crystal microbalance); (e) cantilever biosensor with the aptamer modified at the tip and change in deflection can be detected as the aptamer binds the target present on the sample surface; and ( $f$ ) electrochemical sensors where change in current upon aptamer target binding can be measured.

Figure 2.1: A typical: (a) AT-cut quartz crystal; and (b) sheering as a result of applied electrical potential. In the case of quartz, this sheering is reversible ${ }^{86}$.

Figure 2.2: A typical QCM quartz crystal showing two electrodes (gold and grey) deposited on each face of the quartz (white)

Figure 2.3: Butterworth Van Dyke electrical model showing the motional arm with inductor (L), Resistor (R), Capacitor (Cs), and parasitic Capacitance (Cp).

Figure 2.4: A sample surface being bombarded with primary ions causing the surface to fracture/deform and release secondary ions into the gas phase. The secondary ions may be charged or neutral and may fragment once in the gas phase. Post-bombardment bombardment in the gas phase can excite neutral fragments.

Figure 2.5: A typical high resolution ToF-SIMS instrument showing a primary ion source (1) to bombard the sample surface, an electron impact source (2) used in traditional ToF-MS, the target (3), reflectron in the ToF tube (4), and the MS detector (5). In this ToF-SIMS an optional post-ionization ion source is also added (6) to ionize neutral fragments in the gas phase.

Figure 2.6: Example of different modes of ToF-SIMS depending on operational needs: (a) bunch mode showing high mass resolution; (b) burst alignment showing high spatial resolution; and (c) 
burst mode, used when information on both mass and spatial resolution is needed without maximum resolution of each.

Figure 2.7: Typical single beam UV/vis spectrophotometer showing the light source (A), the grating which selects specific wavelengths for the sample (B), aperture (C), sample (D), and detector (E).

Figure 2.8: (a) Graphical representation of coherent (in phase) and incoherent (out of phase) light; and (b) typical setup of a light microscope. In contrast, an inverted microscope has the objective below while the light source and condenser lens are situated above the stage.

Figure 2.9: Graphical representation of resolution, where in (a) and (b) two separate points cannot be resolved, while in (c) the limit of resolution is approaching where the two points are becoming increasingly distinguishable.

Figure 2.10: Typical setup for a confocal microscope, where light generated (by laser or lamps) passes through an entrance pinhole (A) to remove out of phase light. The light then passes through a dichromatic mirror $\left(M_{1}\right)$ which splits and allows only selected wavelengths (by user) to pass through. The light is then focused by an objective $(O)$ onto the focal plane (D) of the specimen (S). The reflected light is then sent through the objective to the dichromatic mirror which reflects selected wavelengths (by user) onto an exit pinhole (B) which filters incoherent light. The resultant light is captured by a photocell or a charge coupled device $(P)$ to generate an image of relative light intensities over 2-dimensional space. Moving the focal plane in the axial direction by moving the stage (S), different axial regions of the sample can be imaged ('slices). These slices can be combined to generate a 3-dimentional image of the sample ${ }^{113}$.

Figure 2.11: Experimental Overview. A) Thin film composition. In PDDA/PSS/SA, 5 bilayers of PDDA (blue) and PSS (red) are followed by 5 bilayers of PDDA and SA (green). The films are capped with a final bilayer of PDDA/PSS. The films PDDA/PSS/CT and PDDA/PSS/RO are prepared with a similar composition except that Calf Thymus DNA (CT) or random oligonucleotide DNA (RO) is substituted for SA in the second 5 bilayers. PDDA/PSS films without any DNA were also prepared and used as controls. The film illustration was modified from reference 1. B) Components of the Experiment. The sequence of the random oligonucleotide $(R O)$, the sequence and structure (showing the predicted g-quadruplex) of the sulforhodamine $B$ aptamer (SA), and structures of the polyelectrolytes, PSS and PDDA, are presented. The structures of the target, Sulforhodamine $B$ (SB) and Tetramethylrosamine (TMR; a related dye with no affinity for the Sulforhodamine aptamer) are also shown.

Figure 2.12: AFM image of films terminated with CT (left) and SA (right) showing the different morphologies of the two films. 
Figure 2.13: AFM of (a) PDDA/PSS; and (b) PDDA/PSS/SA films. An increase in surface roughness (bright regions) can be seen in the PDDA/PSS/SA films, correlating with the AFM data shown in Figure 2.9(b).

Figure 2.14: Overlay of UV-Vis spectra of the polyelectrolyte films. DNA containing films are normalized at $260 \mathrm{~nm}$. Black: PDDA/PSS/SA exposed to Sulforhodamine B (SB) Red: PDDA/ PSS/RO exposed to SB, Green: PDDA/PSS/CT exposed to SB, Yellow: PDDA/PSS alone exposed to SB, Blue: PDDA/PSS/SA exposed to Tetramethylrosamine (TMR), Orange: PDDA/PSS/SA exposed to $\mathrm{SB}$, but without $\mathrm{K}^{+}$. The regions of the spectra assigned to DNA and dye absorbances are highlighted. Upon immersion of the films in the dye solution, the aptamer films show the greatest degree of dye binding as evidenced by the largest peak at $570 \mathrm{~nm}$.

Figure 2.15: Confocal microscope images of the polyelectrolyte films after dye binding. Excitation $556 \mathrm{~nm}$, emission $575 \mathrm{~nm}$. White bars correspond to $10 \mu \mathrm{m} \mathrm{A)} \mathrm{PDDA/PSS/RO} \mathrm{B)}$ PDDA/PSS/CT C) PDDA/PSS/SA. The random oligonucleotide-containing film (PDDA/PSS/RO -box A) shows few bright spots associated with the dye fluorescence. PDDA/PSS/CT films show very little dye fluorescence, with some localized non-specifically within defects on the film surface. The film containing the aptamer (PDDA/PSS/SA -box C) shows the brightest fluorescent areas associated with bound dye.

Figure 2.16 : Binding affinity curve using UV/vis of PDDA/PSS/SA films with varying concentrations of target $(\mathrm{SB})$. The binding affinity using $\mathrm{UV} / \mathrm{vis}$ ranged from $0.8 \mu \mathrm{M}$ to $3 \mathrm{mM} \ldots . . .67$

Figure 2.17: (a) Control QCM dissociation constant experiment on a PDDA/PSS/CT film. The line represents the fit to a single $K_{d}$ model. Inset: Values calculated using the single $K_{d}$ model vs. the experimental data.; (b) Representative data from QCM dissociation constant experiments. A PDDA/PSS/SA film on a quartz crystal was exposed to solutions of sulforhodamine $B$ dye and the relative frequency was plotted vs. the dye concentration. Top: Raw frequency data from the QCM experiment. Bottom: Plot of frequency over dye concentration. The line represents a two $K_{d}$ model (see text). Inset: Values calculated using the two $K_{d}$ model vs. the experimental data.

Figure 2.18: A TOF-SIMS sputter depth profile of PDDA/PSS/SA before (A) and after (B) dye binding. Red: $\mathrm{SO}_{2}{ }^{-}$, Green: $\mathrm{PO}_{3}^{-}$; Blue; $\mathrm{SiO}_{2}^{-}$. The $\mathrm{SO}_{2}^{-}$secondary ion is used to trace both $\mathrm{PSS}$ and $\mathrm{SB}$, while the $\mathrm{PO}_{3}$ secondary ion is used to track the presence of the aptamer. The $\mathrm{SiO}_{2}{ }^{-1}$ ion counts have been reduced by a factor of 10 to fit on the same scale as the $\mathrm{SO}_{2}{ }^{-}$. A) PDDA/PSS/SA alone: $\mathrm{SO}_{2}{ }^{-}$drops off dramatically as $\mathrm{PO}_{3}{ }^{-}$rises, and then rises again in the area directly above the slide surface. This trend was expected as the aptamer replaces PSS in the middle 5 bilayers of this film. B) PDDA/PSS/SA and dye: $\mathrm{SO}_{2}{ }^{-}$counts reach a maximum at the same depth as do the $\mathrm{DNA}^{\prime} \mathrm{PO}_{3}$ counts, suggesting that the dye and the DNA are co-localized. 
Figure 3.1: Typical FRAP experiment where a small fluorescent spot (ca. 1-2 $\mu \mathrm{m}$ ) is photobleached within a capsule $(>2 \mu \mathrm{m})(1 \rightarrow 2)$ and the resultant recovery of the fluorescence can be monitored over time (3). FRAP experiments can provide information on diffusion and kinetics of different mobile phases in the sample.

Figure 3.2: SEM micrographs of PSS doped $\mathrm{CaCO}_{3}(\mathrm{a})$ cubes; and (b) spheres. The spheres were formed under very high stirring (high shearing) and a longer stirring time.

Figure 3.3: Figure : SEM micrograph of PDDA/PSS Ibl films after core dissolution. SEM performed on a gold/palladium coated sample. There was no presence of microcapsules in the sample. ...86

Figure 3.4: SEM micrograph of Ibl films template on a PSS doped $\mathrm{CaCO}_{3}$ cores with $(\text { PDDA })_{4}(\text { PSS })_{4}$. No microcapsules were seen in the micrographs.

Figure 3.5: (a) SEM micrograph of PSS doped PAH/PSS-aptamer microcapsules (PAH) $)_{4}(\text { SA })_{2}(\mathrm{PSS})_{2}$ with an average size of ca. $2 \mu \mathrm{m}$ [dry]; (b) CLSM of fluorescein labeled aptamer microcapsules $(\mathrm{PAH})_{4}(\mathrm{SA})_{2}(\mathrm{PSS})_{2}$ (left, 489nm laser); and (c) unlabelled sulforhodamine $B$ filled microcapsules $(\mathrm{PAH})_{4}(\mathrm{SA})_{2}(\mathrm{PSS})_{2}$ (right, $561 \mathrm{~nm}$ laser) [wet]. Microcapsules in CLSM study had an average size of ca. $8 \mu \mathrm{m}$.

Figure 3.6: (a) CLSM image of $\mathrm{CaCO}_{3}$ cores shown in the light channel (right), indicating lack of fluorescence (fluorescence channel, left); and (b) theoretical modeling after careful alignment of CLSM optics to avoid any overlap from the two emissions of the fluorophores.

Figure 3.7: Fluorescence co-localization experiments to confirm aptamer-target binding and the requirement for $\mathrm{K}^{+}(\mathrm{a}-\mathrm{c})$ Series of binding experiments where $\mathrm{K}^{+}$was excluded from the solution; (d-f) Series of binding experiments with $K^{+}$present in solution. (A) and (D) Emission image from the green channel displaying fluorescein emission from the tagged SA within the microcapsule walls (in green); (B) and (E) Emission image from the orange channel showing the presence of the sulforhodamine B target; $(C)$ and $(F)$ Overlay of orange and green channels, where yellow emission is the result of the colocalization of SA and the sulforhodamine target. It is shown that in the presence of a monovalent cation $(F)$ there is a high degree of colocalization of the aptamer and the target, as indicated by the yellow color. In the absence of the cation, only a small degree of colocalization can be detected, while the majority of the green and red emission can be seen separately. This suggests a small degree of non-specific binding to the polyelectrolyte microcapsule occurs but that the activity of the aptamer and the majority of the binding of the target to the microcapsule requires $K^{+}$. Scale bars are $10 \mu \mathrm{m}$.

Figure 3.8: (a) CLSM image of microcapsules filled or partially filled with $\mathrm{CaCO}_{3}$ with fluorescent channel on left and light channel on right; (b) FRAP on these microcapsules shows little recovery of the target (sulforhodamine B) fluorescence intensity (left), where the red spot on the right 
indicates the bleach spot. The aptamer was not labeled with fluorescein in the FRAP experiments.

Figure 3.9: (a) FRAP of microcapsules without $\mathrm{CaCO}_{3}$, showing the photobleaching and measurement of the subsequent fluorescence recovery in seconds; and (b) raw FRAP data after first-order kinetics fit using the microscope software 'Kinetic'. The $t_{1 / 2}$ from the fit data was used to calculate the diffusion coefficient. Figure (b) is not corrected for bleach spot size and is presented here to show a relative change between the three types of microcapsule conditions tested.

Figure 4.1: Mechanisms of mass-transport limited systems: (a) diffusion limited where ions are governed only by diffusion and not charge attraction; (b) migration limited transport showing attraction of an opposite charge (in this case positive) to the electrode and electrostatic repulsion of the like charge (negative); and (c) convection limited where ion mobility is promoted by physical means such as stirring or shaking ${ }^{161}$.

Figure 4.2: 3-electrode cell showing the reference, working, and auxiliary electrodes placed through a Teflon cap and into a fixed volume vial.

Figure 4.3: Triangular cyclic potentials during a typical cyclic voltammetry scan. The slope of the line is the scan rate. The potential is cycled over a period of time and the changes in current measured $^{166}$

Figure 4.4: Cyclic voltammogram of $\mathrm{K}_{3} \mathrm{Fe}(\mathrm{CN})_{6}$; where $i_{p c}$ and $i_{p a}$ are peak cathodic and anodic currents, respectively; and $E_{p c}$ and $E_{p a}$ are cathodic and anodic peak potentials of a reversible system ${ }^{166}$.

Figure 4.5: (a) $\mathrm{CV}$ of bare gold electrode after polishing and $1 \mathrm{M} \mathrm{H}_{2} \mathrm{SO}_{4}$ etch; and (b) $\mathrm{CV}$ of different number of bilayers (PAH/PSS) with $0.5 \mathrm{mM}$ ferricyanide: purple ( 2 bilayers); green ( 4 bilayers); and blue ( 6 bilayers) showing decreasing current on the electrode as a result of increasing number of layers.

Figure 4.6: $\mathrm{CV}$ of bilayer films with (A) $\mathrm{K}_{3} \mathrm{Fe}(\mathrm{CN})_{6}$ and $(\mathrm{B}) \mathrm{Ru}\left(\mathrm{NH}_{3}\right)_{6} \mathrm{Cl}_{3}$ before (blue) and after (red) annealing with semi-derivative analysis (inset).

Figure 4.7: Normalized Diffusion coefficients $\left(\mathrm{cm}^{2} / \mathrm{s}\right)$, before and after annealing, with $\mathrm{K}_{3} \mathrm{Fe}(\mathrm{CN})_{6}$ of aptamer film (SA; blue); random DNA film (RO; green); and synthetic films with no DNA (PSS; red) in the absence of target or control dye (control); presence of control dye (TMR); and target (SB) $[$ Red = PSS; Green = RA; Blue = SA . 125

Figure 4.8: Normalized Diffusion coefficients $\left(\mathrm{cm}^{2} / \mathrm{s}\right)$, before and after annealing, with Ru $\left(\mathrm{NH}_{3}\right)_{6} \mathrm{Cl}_{3}$ of aptamer film (SA; blue); random DNA film (RO; green); and synthetic films with no 
DNA (no DNA; red) in the absence of target or control dye (control); presence of control dye $(T M R)$; and target $(S B) .{ }^{* *}$ indicates significant difference $(p<0.05)$ between the three films and $T M R$ and $S B$ of the SA film. [Red = PSS; Green = RA; Blue = SA]

Figure 5.1: Jablonski diagram showing transitions from $S_{0} \rightarrow S_{n}$ and $S_{n} \rightarrow S_{0}$ on the left and the transitions $T_{n} \rightarrow T_{0}$ on the right.

Figure 5.2: Fluorescence spectrum (bottom) showing absorbance transitions $\left(S_{0} \rightarrow S_{n}\right)$ which is represented by valleys and the emission transitions $\left(S_{n} \rightarrow S_{0}\right)$ which are represented by peaks. The overlap between the absorption and emission spectrum is the Stokes shift.

Figure 5.3: Typical setup to study fluorescence anisotropy. This image is taken from the technical notes of the manufacturer of the instrument used in our laboratory. .138

Figure 5.4: Michelson interferometer, where the incoming light is split with a beam splitter. $50 \%$ of the light is sent to the sample $\left(M_{2}\right)$, while the remaining $50 \%$ is reflected of a mirror $\left(M_{1}\right)$. The change in frequency between $M_{1}$ and $M_{2}$ is compared, which gives an indication of the change in frequency of motion. Considering the same reduced mass, the changes in vibrational motion ( $k$ in figure 5.4) which are characteristic of different chemical environments (at resonance) will give rise to diagnostic vibrational frequencies of motion.

Figure 5.5: Structures of (a) L-lysine [left]; and (b) L-histidine [right] 144

Figure 5.6: Desalting/filtration tubes where the solution is spun in a centrifuge and materials larger than the filter molecular weight cut-off is retained while all smaller molecules are eluted. Some non-specific binding to the filter itself is expected during filtration. The image was adapted from the manufacturer for desalting tubes used in our laboratory.

Figure 5.7: (a) raw FTIR spectrum of filtrate after aptamer-target incubation and subtraction of solvent background (water). Peak centered at ca. $1600 \mathrm{~cm}^{-1}$ was considered to be from the primary amine. Percentage area was determined for the amine peak by using FTIR software. (b) $K_{d}$ data fitting for the aptamer by removing four data points with the correlation between predicted and observed data shown in the inset. Figure (b) shows a rough approximation of where a $\mathrm{K}_{\mathrm{d}}$ might lay. As the concentration is increased of the target, less quantity of target ( $\mathrm{L}$ lysine) is eluting through the filter until there is a significant decrease in quantity of target eluted ca. $2 \times 10^{-6} \mathrm{M}$ which could be an expected $K_{d}$.

Figure 5.8: Anisotropy data shown after ca. fifteen minute aptamer-target incubation in 0.1M $\mathrm{KCl}$, with the correlation between observed and calculated anisotropy's in the inset. 
Figure 5.9: Binding affinity curve after 24 hour incubation with the correlation between observed and calculated anisotropy shown in inset. The $K_{d}$ was calculated to be $4 \times 10^{-6} \mathrm{M}$ with a $\mathbf{R}^{2}=0.99$. It is important to note that the actual $K_{d}$ calculated may be higher because we did not test more points at higher concentrations, and that the $K_{d}$ calculated from this curve was used simply to optimize the diffusion experiments.

Figure 5.10: Confocal microscopy image after core dissolution with $0.5 \mathrm{M} \mathrm{NaCl}$ as supporting electrolyte. We found no presence of microcapsules in the fluorescence (left) or light (right) channels. Bars correspond to $10 \mu \mathrm{m}$.

Figure 5.11: Confocal microscope image of: (a) microcapsules with fluorescein tagged aptamer (green; left) and light channel (right); and (b) microcapsules with filled sulforhodamine B (vellow; left) and light channel (right). Red bars are $5 \mu \mathrm{m}$.

Figure 5.12: (a) Typical FRAP experiment in terms of intensity; and (b) raw data not normalized in terms of bleach spot size for L-lysine (black) and L-histidine (brown). While the raw data shows slower recovery of fluorescence after photobleaching, the bleach spot sizes were larger and thus once corrected for spot size, L-lysine had a diffusion ca. $2 X$ higher than L-histidine...161

Figure 6.1: Phase diagram for synthetic polymeric amphiphiles. Phase diagrams are shown as a function of amphiphile concentration vs. water (a) and amphiphile solvent vs. water (b). Both phase diagrams shown that by increasing the molar concentration of water, the change in interstitial stress and hydration drives the formation of different morphologies.

Figure 6.2: Effects of receptor clustering on sensitivity of detection (y-axis) for clusters (solid line) and single receptors (dashed lines) dependant on the number of receptors ( $x$-axis $\times 1000$ ). In this situation, the clusters are expected to be interconnected.

Figure 6.3: Receptor clustering model (theoretical): (a) a ligand (labeled X) is bound to an individual receptor (hexagon) and thus effects (activates/inactivates) only the one receptor, while (b) a ligand bound to one receptor effects a whole cluster (circle marked by dashed lines), thereby, increasing the detection sensitivity (signal strength). In the case of aptamers, an activated state is a free aptamer while inactivated states describe aptamers bound to targets. The white and grey hexagons refer to a random distribution of active and in active receptor sites used in modeling ${ }^{230}$

Figure 6.4: Theoretical effect of activity spread (higher probability of effecting neighboring receptor molecules) on detection of model ligand for one (triangles), two disjointed (crosses), three disjoined (circles), and four disjointed (squares) clusters ${ }^{230}$. 
Figure 6.5: C12-SA conjugate used in this project showing the $C_{12}$ hydrophobic tail and polar DNA head.

Figure 6.6: (a) 12\% PAGE (90min; 45mA) of crude $\mathrm{C}_{12}$-SA in all lanes showing the product in band 1 (white box); and (b) ESI-MS of $\mathrm{C}_{12}$-SA product (white box in (a)).

Figure 6.7: Strategy for aptamer aggregate preparation. Rehydration and sonication of an aptamer sample, after deposition onto a glass surface, yields aptamer aggregates of varying size and morphology.

Figure 6.8: $\mathrm{SEM}$ of $\mathrm{C}_{12}-\mathrm{SA}$ aggregates formed. Sizes range $>100 \mathrm{~nm}$ with various shapes of aggregates.

Figure 6.9: Characterization of the Aptamer Aggregates by AFM. AFM images (left) show that $\mathrm{C}_{12}$-SA aggregates in the size range of less than $100 \mathrm{~nm}$ are present (Distributions are shown on the right) ( $a$ and $b$ ). Larger aggregates, in the range of $100-300 \mathrm{~nm}$ were also seen (c)

Figure 6.10: Extruder set-up used for size fractionation. Membranes of different sizes can be used to fractionate different sized particles. Membranes are based on size cut-offs.

Figure 6.11: Apparent dissociation constant measurements before and after extrusion of the aggregate samples. Changes in the fluorescence anisotropy of Sulforhodamine $B$ upon binding at different aptamer concentrations were used to determine the apparent $K_{d}$ values for $C_{12}-S A$ either in aggregated or free forms. Anisotropy data is shown as percent binding vs. free DNA concentration in order to better compare the different samples. The error on the concentration ranged from $\pm 1 \%$ for the first dilution to $\pm 3 \%$ for the most dilute serial concentration. For clarity, we did not measure aptamer concentrations down to $\mathrm{pM}$ range, rather we used our dye as a reporter molecule $(1 \mu \mathrm{M}$; emission intensities of $>10,000 \mathrm{cps})$ to measure changes in anisotropy upon binding with the differing concentrations of the nano-aggregates (as seen in graph). Lines of best fit were calculated as described in Experimental. Free alkyl aptamer (blue diamonds) has the highest apparent $K_{d}$ at ca. $100 \mathrm{nM}$. Before extrusion, the polydisperse sample (orange squares) has an apparent $K_{d}$ of ca. $300 \mathrm{pM}$. Extrusion to separate the aggregates into those smaller than $100 \mathrm{~nm}$ (red circles) and those larger than $100 \mathrm{~nm}$ (green triangles) reveals that the smaller sized fraction has an intermediate apparent $K_{d}$ of ca. $10 \mathrm{nM}$, while the larger sized aggregates have an average apparent $K_{d}$ of $0.3 \mathrm{pM}$

Figure 6.12: Anisotropy curve for $>100 \mathrm{~nm}$ aggregate fraction with negative control dye TMR. The apparent $K_{d}$ estimated here $\left(1 \times 10^{-7} \mathrm{M}\right.$, which may be even higher $)$ is six orders of magnitudes higher than that for the same fraction using the target dye, thereby confirming that scatter is not responsible for the binding affinity enhancement in the large fractions. 


\section{List of Tables:}

Table 2-1: Comparisons of Dye, Polymer, and DNA Absorbance Values for the Films of this Study.

Table 3-1: Diffusion Coefficients obtained by FRAP experiments on the microcapsules. . .63

.101 


\section{List of Acronyms}

AFM

ATP

CLSM

CV

DLS

DMF

DNA

DPV

EDTA

FDA

FITC

FRAP

FTIR

$K_{d}$

LB

LSCM

NA

NMR

NUE

OECD

PAGE

PAH
Atomic Force Microscopy

Adenosine Triphosphate

Confocal Laser Scanning Microscopy

Cyclic Voltammetry

Dynamic Light Scattering

Dimethyl formamide

Deoxyribonucleic Acid

Differential Pulse Voltammetry

Ethylenediaminetetraacetic acid

Food \& Drug Administration (USA)

Fluorescein isothiocyanate

Fluorescence Recovery After Photobleaching

Fourier-Transform Infrared Spectroscopy

Binding affinity where half of the aptamers are bound to target

Langmuir-Blodgett

Laser Scanning Confocal Microscopy

Numerical Aperture

Nuclear Magnetic Resonance spectroscopy

Nutrient Uptake Efficiency

Organization for Economic Co-operation and Development

Polyacrylamide Gel Electrophoresis

Poly(allylamine) hydrochloride 
PCR Polymerase Chain Reaction

PDDA Poly(diallyldimethyl ammonium) chloride

PEM Polyelectrolyte Multilayers

PSS Poly(styrene sulfonate)

QCD-D Quartz Crystal Microbalance - Dissipation

QCM Quartz Crystal Microbalance

RNA Ribonucleic Acid

RO random oligonucleotide

RT Room Temperature

SA Sulforhodamine B aptamer

SB Sulforhodamine B dye

SELEX Systematic Evolution of Ligands by Exponential enrichment

SEM Scanning Electron Microscopy

SOM Soil Organic Matter

TMR Tetramethylrosamine

VEGF Vascular Endothelial Growth Factor 


\section{Chapter 1. Introduction}

DeRosa, M.; Monreal, C.; Schnitzer, M.; Walsh, R.; Sultan, Y. Nature Nanotechnology, 2010, 5, 91.

\subsection{Nanomaterials}

There still remains no international consensus on the definition of nanomaterials; however, all definitions in literature or by government agencies have some commonalities. There is a differentiation made between intentional (or manufactured) and incidental nanomaterials (e.g., combustion by-products or naturally derived minerals). Manufactured nanomaterials (nanomaterials hereafter) are synthesized to manipulate some nanoscale property and are by and large the area of interest in modern nanotechnology. Nanomaterials are generally described to have at least one spatial dimension between $1-100 \mathrm{~nm}$ (the nanoscale) ${ }^{1}$. At this scale, three phenomena primarily result in the unique properties seen in nanomaterials relative to their bulk and molecular counterparts. First, the specific surface area of materials increases exponentially as the nanoscale is approached ${ }^{2}$. This results in a greater proportion of surface atoms and, in many cases, increased reactivity. For example, gold, while being chemically inert on the bulk scale, is an efficient catalyst for the oxidation of carbon monoxide on the nanoscale ${ }^{3}$. Second, in semi-conductors, as the particle approaches the Bohr radius ${ }^{4}$, the confinement of the atoms yields unique quantum effects resulting in increased band gap energies and quantized energy levels. This confinement also causes the electrons to be very energetic and reactive, thereby imparting a certain level of 
unpredictability in properties. Third, surface plasmon resonance in metal nanoparticles, which is the result of the particle size and the collective oscillation of the surface electrons as they interact at resonance with the electromagnetic wave, result in unique properties relative to bulk particles $(>100 \mathrm{~nm})^{5}$. For example, gold particles display a red to blue shift in optical absorbance as the particle size is increased within the nanoscale, while bulk gold particles display size independent optical absorbance ${ }^{6}$. Moreover, the mere fact of being on the nanoscale allows for increased biological scale interactions since many biological systems have nano-porous systems (such as channels or membranes) with which nanoparticles can interact. These unique properties can either lead to adverse impacts to the environment and/or organisms as the potential fate and effects of these nanoparticles cannot be extrapolated from bulk particles or lead to positive impacts such as the fertilizer system described herein. This thesis will examine the feasibility of a nanomaterial-based system for the triggered delivery of fertilizers, an 'smart fertilizer'.

\subsection{Smart Fertilizers}

Nitrogen, which is a key nutrient source for food, biomass and fibre production in agriculture, is by far the most important element in fertilizers when judged in terms of the energy required for its synthesis, tonnage used and monetary value. However, compared with amounts of nitrogen applied to soil, the nitrogen use efficiency (NUE) by crops is very low. In conventional fertilizers, between 50 and $70 \%$ of the nitrogen 
applied is lost owing to leaching in the form of water soluble nitrates, emission of gaseous ammonia and nitrogen oxides, and long-term incorporation of mineral nitrogen into soil organic matter (SOM) by soil microorganisms ${ }^{7}$. Current methodologies to increase NUE include the development of slow/controlled release fertilizers.

Slow/controlled release fertilizer methodologies include: (1) water soluble fertilizers with slow/controlled release due to a matrix barrier (e.g., embedded in a polymer matrix); (2) inorganic fertilizers of low solubility (e.g., inorganic ammonium phosphates); (3) low solubility materials able to biodegrade or chemically degrade (e.g., urea); and (4) materials with moderate solubility with gradual biodegradation in the soil matrix ${ }^{8}$. One of the largest fertilizer Companies in North America, Agrium, has a patented slow release system on the market, based on a polymer-and sulfur-coated urea platform. All these fertilizers are based on the notion that by adding coatings or matrices to fertilizers, the release can be slowed down to increase its presence in the soil and reduce waste. However, all these systems lack the ability to be smart, that is, to release fertilizer on demand only when required by the crop. Numerous attempts to increase the NUE, including the ones abovementioned, have so far met with little success, and the time may have come to apply nanotechnology to solve some of these problems. There are many examples of the utilization of nanoscale functionality in plant biology. For example, plant roots contain pores between one and tens of nanometers ${ }^{9}$ which are important for the transport of ionic and molecular species ${ }^{10}$. Plant leaves also have nanoscale structure, such as cutical and stomatal pores which can facilitate nanoparticle penetration ${ }^{11}$. Once in the cell, nanoparticles can transport through cells via 
plasmodemata, which have channels $50-60 \mathrm{~nm}$ in diameter ${ }^{12}$. More recently, carbon nanotubes were shown to penetrate tomato seeds ${ }^{13}$, and zinc oxide nanoparticles were shown to enter the root tissue of ryegrass ${ }^{14}$ (Figure 1.1).
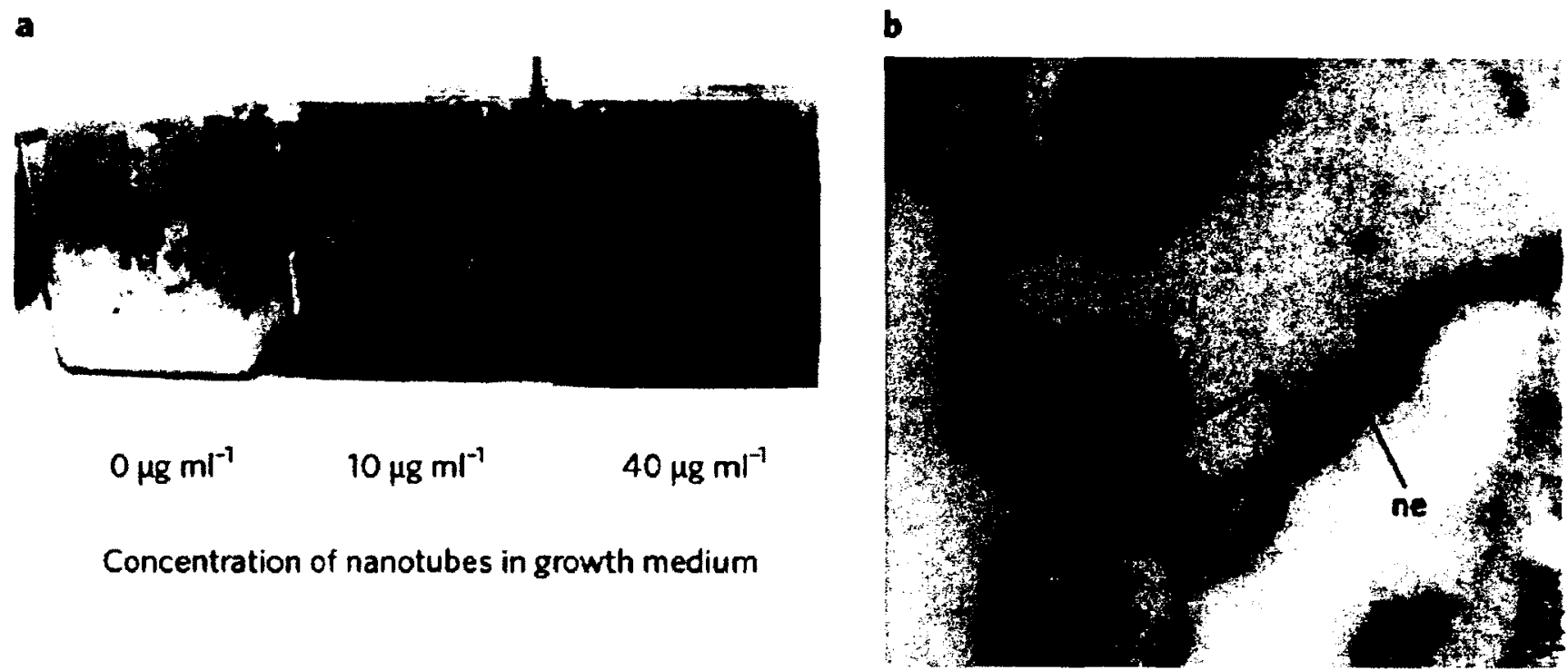

Figure 1.1: Nanomaterials can enter plants. (a) improved growth of 27 day old tomato seedling growth in a medium containing carbon nanotubes. (b) Transmission electron micrograph of ryegrass roots showing the entry of zinc oxide nanoparticles (ni, nucleus; ne, nuclear envelope; np, nanoparticles).

This suggests that new nutrient delivery systems that exploit the nanoscale porous domains on plant surfaces can be developed. The potential use of nanotechnology to improve fertilizer formulations, however, may have been hindered by reduced research funding and the lack of clear regulations and innovation policies. Current patent literature shows that the use of nanotechnology in fertilizer development remains relatively low (about 145 patents and patent applications between 1998 and 2011) 
compared with pharmaceuticals (more than 8,000 patents and patent applications over the same period) $)^{15}$.

A nanofertilizer refers to a product that delivers nutrients to crops in one of three ways.

The nutrient can be encapsulated inside nanomaterials such as nanotubes or nanoporous materials, coated with a thin protective polymer film, or delivered as particles or emulsions of nanoscale dimensions. Owing to a higher surface area to volume ratio, the effectiveness of nanofertilizers may surpass the most innovative polymer-coated conventional fertilizers, which have seen very little improvement in the past ten years.

Ideally, nanotechnology could provide devices and mechanisms to synchronize the release of nitrogen (from fertilizers) with its uptake by crop; the nanofertilizers should release the nutrients on-demand while preventing them from prematurely converting into chemical/gaseous forms that cannot be absorbed by plants. This can be achieved by preventing nutrients from interacting with soil, water and microorganisms, and releasing nutrients only when they can be directly internalized by the plant.

Examples of these strategies are beginning to emerge. Zinc-aluminum-layered doublehydroxide nanocomposites have been used for the controlled release of chemical compounds that regulate plant growth ${ }^{16}$. Improved yields have been claimed for fertilizers that are incorporated into cochleate nanotubes (rolled-up lipid bilayer sheets) ${ }^{17}$. The release of nitrogen by urea hydrolysis has been controlled through the insertion of urease enzymes into nanoporous silica ${ }^{18}$. Although these approaches are 
promising, they lack the mechanisms that can recognize and respond to the needs of the plant and changes in nitrogen levels in the soil. The development of functional nanoscale films ${ }^{19}$ and devices has the potential to produce significant gains in the NUE and crop production. In addition to increasing the NUE, nanotechnology may be able to further improve the performance of fertilizers in many other ways. For example, owing to its photocatalytic property, nanosize titanium dioxide has been incorporated into fertilizers as a bactericidal additive. Moreover, titanium dioxide may also lead to improved crop yield through the photoreduction of nitrogen gas $^{20}$. In addition, nanoscale particles absorbed by roots have been shown to form films at the cell walls, which can enhance the plant's resistance to stress and lead to improved yields ${ }^{21}$. Clearly, opportunity exists for nanotechnology to have a profound impact on energy, the economy and the environment, by improving fertilizer products. New prospects for integrating nanotechnologies into fertilizers should be explored, whilst being cognizant of any potential risk(s) to the environment or to human health. With targeted efforts by governments and academics in developing such enabled agri-products, we believe that nanotechnology will be transformative in this field.

The primary motivation for this thesis is to work towards developing a smart fertilizer to maximize NUE by releasing nitrogen on demand only at times when required by the crop during its growth cycle. In our system, the fertilizer will be protected by a smart coating with an embedded biosensor which will only release nitrogen only when required by the crop. The trigger for the release will be a molecule chosen within 
exudates (biochemical signals) released by the plant into the soil to stimulate nitrogen uptake when required for growth. In the absence of this triggering or target molecule, the fertilizer will be protected and stable in the solid matrix. Exudates are signaling molecules excreted by the plant (host) at various stages of development ${ }^{22}$. For example, exudates can mediate plant to plant communication, stimulate microbial communities in or around the rhizosphere to prompt release of nutrients such as nitrogen, and/or repel one organism while attracting another ${ }^{23}$. Figure 1.2 shows the possible positive and negative interactions of exudates with the soil environment. In this project, we concentrate on the signaling pathway used to stimulate growth, i.e., release of nitrogenous compounds to promote growth of the crop. 


\section{Positive Interactions}

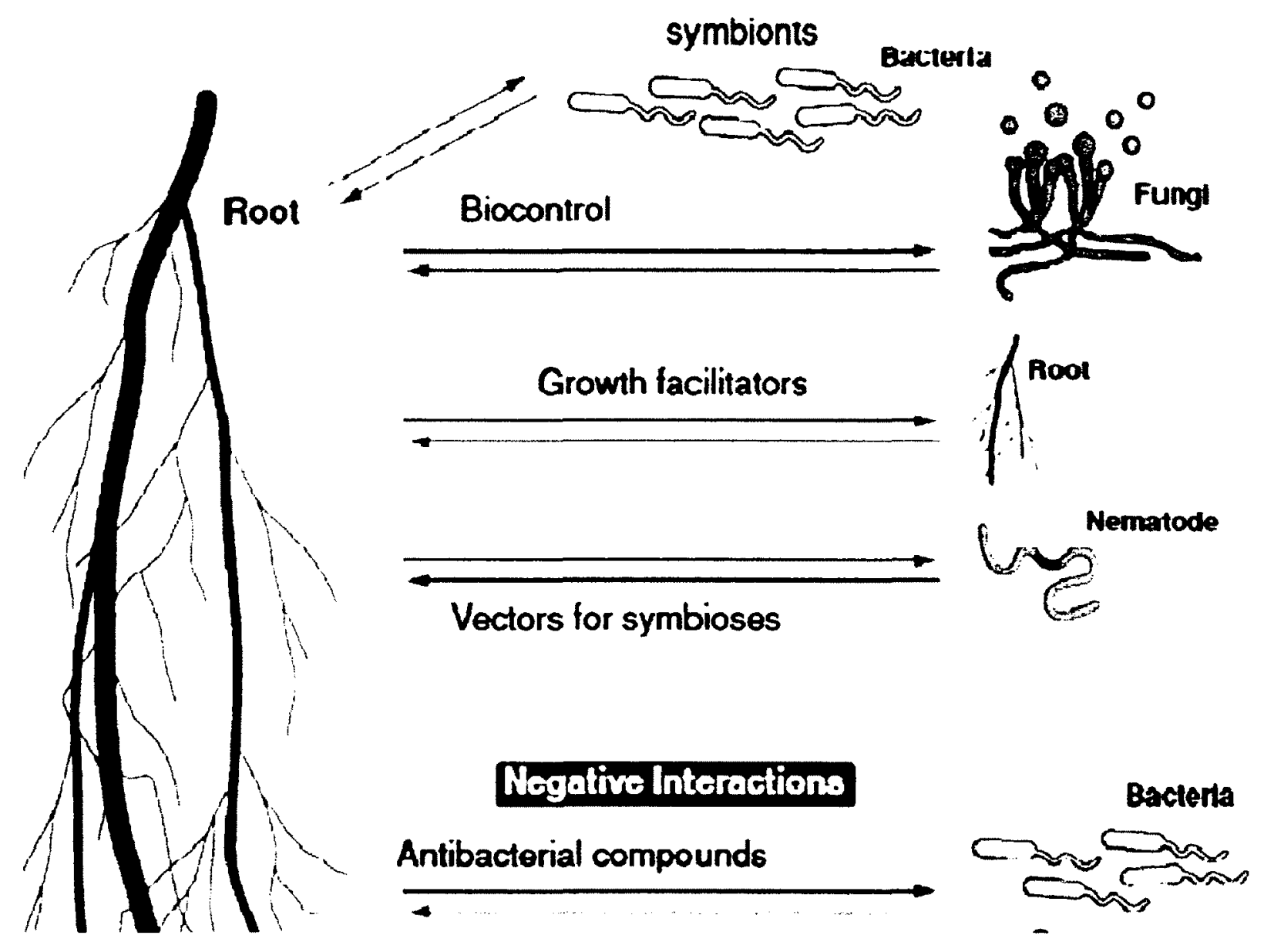

Figure 1.2: Possible positive and negative interactions of exudates with soils ${ }^{23}$.

By creating an on-demand fertilizer system, loss of the nitrogen to the environment will be drastically reduced and NUE dramatically improved. Indeed, this system can also be used in other systems where controlled release is required, such as for drug delivery, therapeutics and diagnostics.

The sensor for the plant exudates needs to be able to (1) recognize the target under environmentally relevant conditions; and (2) lead to some physical change in the coating 
that will allow for the release of the fertilizer. In this thesis, aptamers will be investigated as the (bio)sensor component because they are able to recognize and bind to molecular targets, and many change shape upon binding. The coatings in this thesis will be manufactured of polyelectrolyte multilayers using the layer-by-layer self assembly which has become an attractive route to build films on the nanoscale for biological applications ${ }^{24}$.

\subsection{Polyelectrolyte Multilayers (PEMs)}

Polyelectrolyte Multilayers (PEMs) consist of a bottom-up buildup of sequentially deposited polycationic and polyanionic multiply charged substances (e.g., polymers or aptamers) on a charged substrate ${ }^{25}$. PEMs were originally described in the seminal work by Gero Decher in $1991^{26}$, who deposited amphiphiles on charged substrates. Since then, the field of PEMs has expanded to include a wide array of synthetic and biological polyelectrolytes. Various techniques are available to deposit PEMS on surfaces, the primary ones being Langmuir-Blodgett (LB) and the Layer-by-Layer approach (|b|). Langmuir-Blodgett deposition works typically for amphiphilic molecules, where the molecules are stacked together at the gas-liquid or liquid-liquid interface. The known interaction of the molecules with each medium provides predictability on the subsequent films formed. Monolayers are formed by disrupting the phase boundaries (gas-liquid or liquid-liquid) by a substrate. The substrate will 'lift' a monolayer of the amphiphile at the phase boundary, which can then be repeated for a different 
amphiphile to form bilayers. Controlling conditions such as concentration and surface tension, LB films of different morphologies on the nanometer scale can be formed ${ }^{27}$. Various strategies have also been demonstrated in literature to form LB films of nonamphiphilic molecules by using a two-solvent system ${ }^{28}$. Lbl self-assembly is much more versatile and easy to use relative to LB, requiring only a beaker and substrate (e.g., quartz). An outline of the $\mathrm{Ibl}$ approach is shown below in Figure $1.3^{29}$. In $\mathrm{lb}$ selfassembly, oppositely charged molecules are sequentially adsorbed on a substrate. These charged molecules can be any biological or synthetic molecule which is multiply charged, so monolayers of films can be formed. Synthetic lbl films have been made of almost every charged polymeric system possible and nanoparticles ${ }^{30}$, while biological molecules have included peptides and nucleic acids ${ }^{31}$, viral proteins ${ }^{32}$, and enzymes ${ }^{33}$. A list of commonly used synthetic polymeric polyelectrolytes for use in Ibl self-assembly can be found on the Sigma-Aldrich website ${ }^{34}$. 


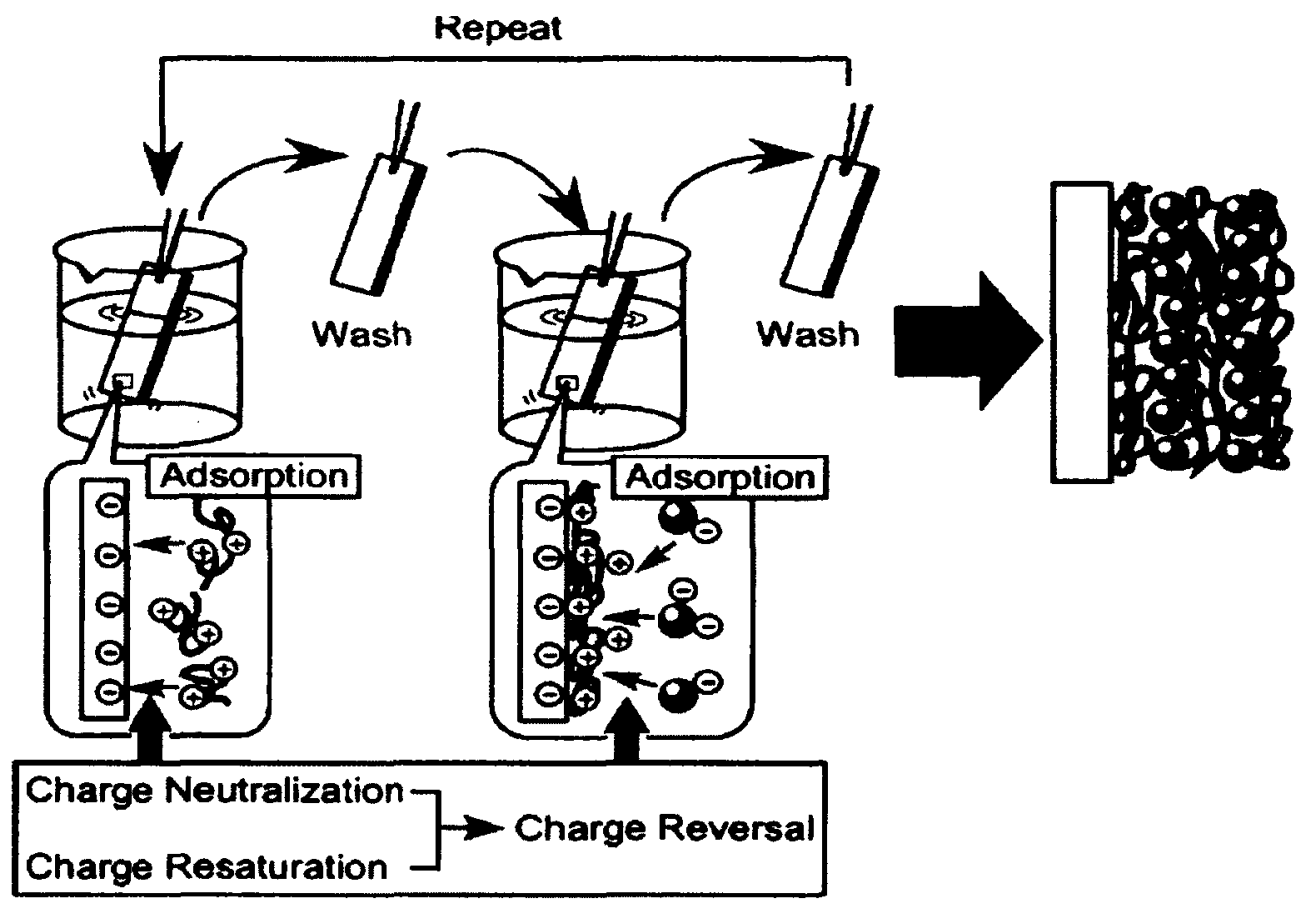

Figure 1.3: Lbl self-assembly. A charged substrate, e..g, quartz is dipped into a solution of oppositely charged polyelectrolyte (black string). After saturation of a monolayer, the film is rinsed copiously with water to remove unbound polyelectrolyte. The film is then dipped in oppositely charged polyelectrolyte to absorb a second layer (grey balls, for clarity).This process can be repeated $n$ times. Charge reversal or overcompensation allows for subsequent films to be deposited. For a two polyelectrolyte system, commonly used nomenclature for $|b|$ films is $(A)_{m}(B)_{n}$, where $A$ and $B$ are the polyelectrolytes, and $\mathrm{m}$ and $\mathrm{n}$ are their respective layers ${ }^{35}$.

The first polyelectrolyte in Ibl self-assembly is deposited on a charged substrate. After saturation, the film is rinsed to remove excess unbound polymer and the substrate is dipped in the oppositely charged polyelectrolyte to deposit the subsequent layer, completing the first 'bilayer'. In this manner, films of various thicknesses can be formed, by manipulating morphologies (monolayers) on the nanoscale. Because only 
polyelectrolytes bound by electrostatic interactions are retained on the film while unbound molecules are removed by rinsing, monolayer deposition is always ensured.

This allows for tailoring of films on the nanoscale, and is the real strength behind PEMs. Layers in PEMs are kept together by extrinsic and intrinsic charge compensation. In intrinsic charge compensation, the positive and negative charges are balanced between the two polyelectrolytes with no interaction from salt ions, whilst in an extrinsic situation the polymer charge is balanced by salt ions present in the deposition solution

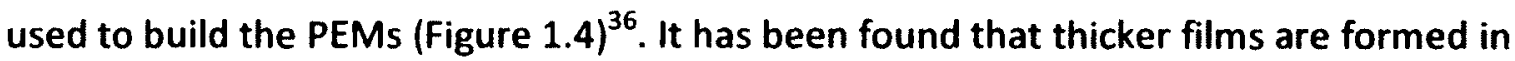
the presence of salt ions because of aqueous swelling and charge overcompensation, whilst no salt produces thin and relatively weakly held films. Adsorption of the layers is driven by the large free energy change $(\Delta G)$ because of co-operative effects ${ }^{37}$ relative to the negative loss in $\Delta G$ as a result of salt ion replacement.
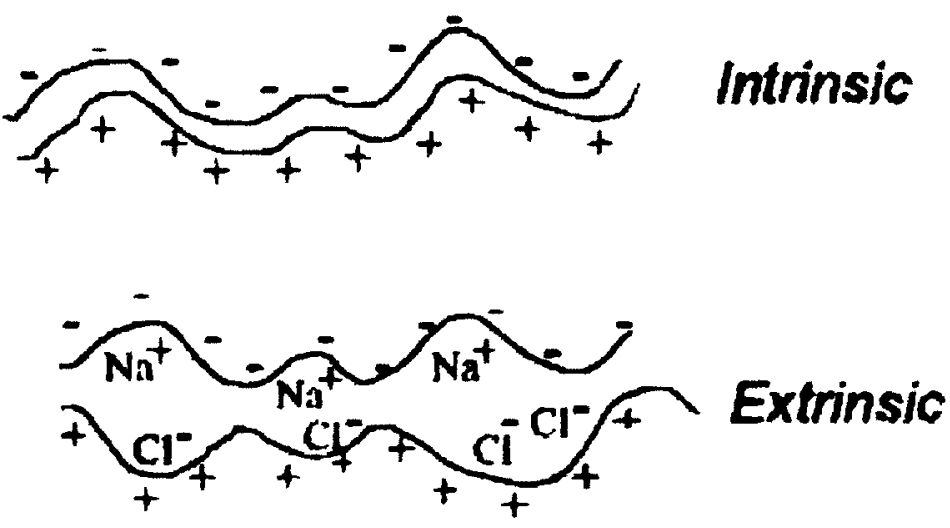

Figure 1.4: Intrinsic and Extrinsic charge compensation, where a 1:1 stoichiometric compensation of charge by only the polyelectrolytes results in an intrinsic compensation and if salt ions partake in the compensation, extrinsic charge compensation occurs ${ }^{38}$. 
The film properties, i.e., thickness, morphology and interpenetration vary with salt concentration $^{39}$, polyelectrolyte concentration, $\mathrm{pH}^{40}$, and temperature ${ }^{41}$. Successive adsorption of each layer is driven by charge overcompensation, i.e., charge inversion. While many groups have attempted to study the mechanisms of charge overcompensation, there are still inconsistencies on whether it is a thermodynamic or kinetic process. We will consider only a thermodynamic process, as it is currently the prevailing theory. As a polyelectrolyte is deposited on the surface, it compensates the films pre-existing charge (from the positive polyelectrolyte) by intrinsic association by a factor $\Phi$. If $\Phi=1$, then the two surfaces are stoichiometrically charge balanced and there is no overcompensation from the film. This is seen with films deposited with no salt, where there is little charge overcompensation and subsequently the PEMs cannot be very thick. Any additional charge on the film is labeled the charge overcompensation, which allows for the adsorption of additional layers, i.e., it allows for charge inversion so the next polyelectrolyte film can sorb. For example, if $\Phi=1.75$, the surface layer is $75 \%$ overcompensated, and subsequently $75 \%$ of the new polyelectrolyte can bind to the existing film. Thus, to have perfect monolayers a charge overcompensation of $100 \%$ is required with every layer added. Charge overcompensation is an associative process of the whole multilayer but only localizes on the surface layer. A general trend in increase of charge overcompensation is seen with increasing salt concentration. This overcompensation is temporarily neutralized by salt ions, which are replaced as the subsequent layers are added. For example, under high salt concentration more polyelectrolyte is able to be retained on the surface by being 'compensated' from the 
salt thus enabling a higher concentration of subsequent layer to be deposited. This can continue as long as there is $100 \%$ overcompensation. Under low salt concentration the overcompensation is low and thus thicker films cannot be formed as the mass of polyelectrolyte diminishes as you increase the number of layers. It has been theoretically and experimentally shown, that the first layer on the substrate has the highest degree of charge overcompensation ${ }^{42}$. The level of charge overcompensation decreases exponentially as the distance between the substrate and film is increased (i.e., increasing the number of films decreases charge overcompensation). Since the overcompensated charge is neutralized temporarily by salt ions, measurement of the amount of salt adsorbed by the film yields a similar exponential decay. Thus, due to this exponential decay behavior, there is a theoretical limit to the thickness of PEMs (number of layers) which can be formed. This limit is highly dependent on the charge density of the polyelectrolytes, salt concentration and the degree of interpenetration of the PEMs. Thermodynamics is presently the most prevalent theory behind charge overcompensation. Overcompensation at the top-most-layer (furthest from the substrate surface) is a balance between the decrease in multilayer degree of freedom and increase in $\Delta G$ from the association between positive and negative charges. Since the polymer density increases as you move from the film surface towards the substrate due to interpenetration, this increases steric repulsion with increasing thickness, and subsequently the level of overcompensation decreases as compensating for the degrees of freedom of the multilayer film by overcompensation becomes energetically unfavorable. Figure 1.5 below shows schematically the effects of charge 
overcompensation. Generally, increasing salt concentration increases film thickness due to higher charge overcompensation and subsequent competitive ion exchange upon addition of oppositely charged polymer ${ }^{43}$. In terms of charge density on each film, it has been found that polymers with low charge density are very weakly held onto the polymer surface. Caruso and co-workers ${ }^{44}$ determined that polyelectrolytes with low charge density can be easily be desorbed into solution, a process attributed to an increase in entropy due to the increase in degrees of freedom.

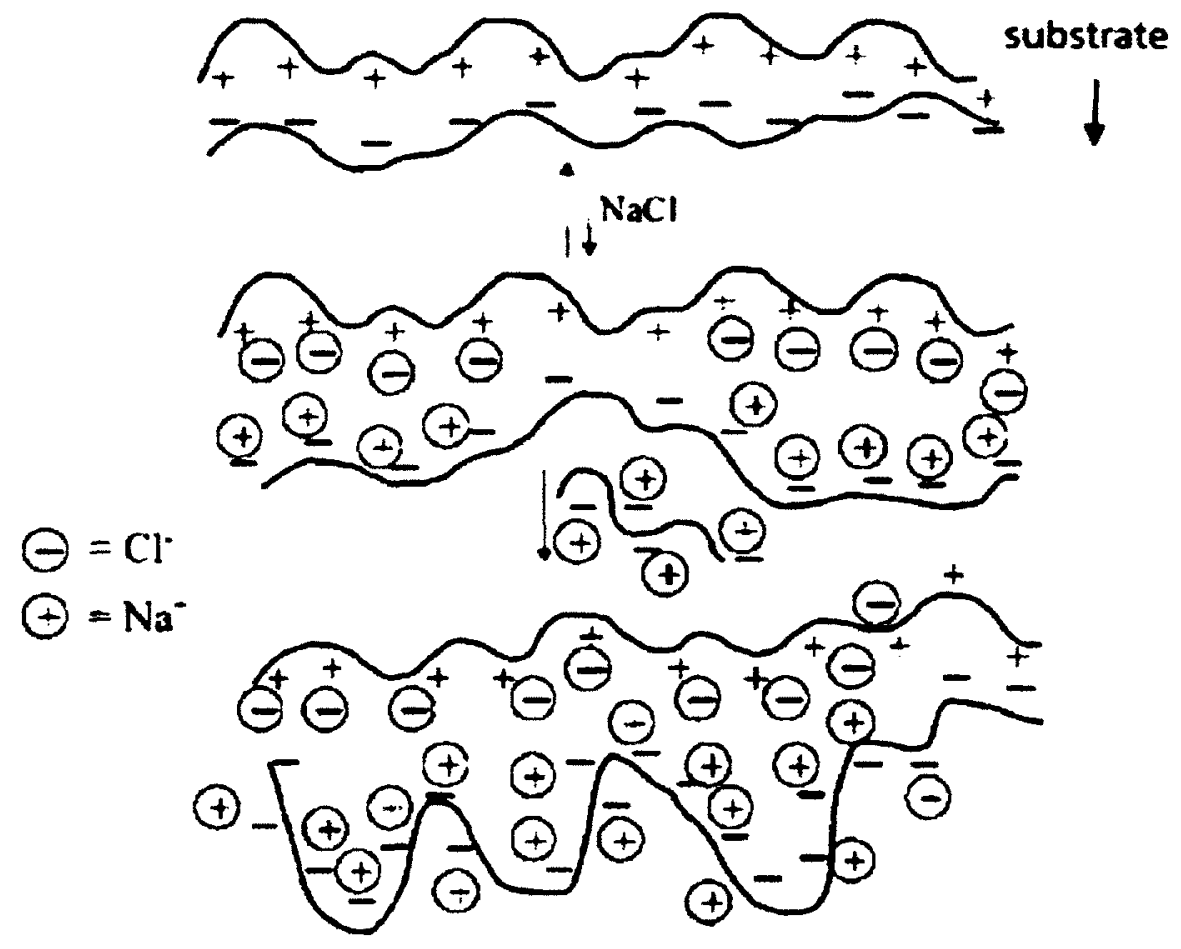

Figure 1.5: Charge overcompensation showing intrinsic compensation (1:1) stoichiometry; (b) introduction of extrinsic charge compensation which causes the film to swell as it allows to decrease steric repulsion still in 1:1 stoichiometry (no charge overcompensation); and (c) in the presence of slightly higher amount of one polyelectrolyte (in this case negative), charge overcompensation can be seen on the surface of the negative polymer overcompensated by the salt ions ${ }^{38}$. 
Due to swelling in aqueous solutions ${ }^{45}$, PEMs are highly porous. Various groups have looked at the diffusion characteristics of these films, both in bilayers ${ }^{46}$ and microcapsules ${ }^{47}$. Pores in the films can be tailored by controlling the salt concentration, allowing for controlled diffusion through these films ${ }^{48}$. These have made PEMs attractive for use in potential selective release systems, such as our smart fertilizer and drug delivery ${ }^{49}$. We decided to use PEMs as they (1) allow for embedding of our aptamer "biosensor" between multilayer films; (2) are porous and thus will allow for the diffusion of our target molecule and subsequent release of our fertilizer; and (3) are stable over an extended period of time.

\subsection{Aptamers}

In 1990, the labs of Joyce ${ }^{50}$, Gold $^{51}$, and Szostak ${ }^{52}$ separately found single stranded DNA and RNA molecules that bound to enzymes, proteins and organic dyes. These are in contrast to double stranded protein binding DNA strands which are naturally found in organisms. These DNA and RNA molecules were termed 'aptamers', latin for 'to fit'. Aptamers are single stranded DNA or RNA molecules which change their spatial 3dimensional conformation upon binding to a target through electrostatic interactions ${ }^{53}$ (see Figure 1.6). These changes in conformation can result in many different secondary structures, such as g-quadruplexes, stem loops, etc. The shape change is driven by a number of non-covalent interactions, such as electrostatics and hydrogen bonding. 


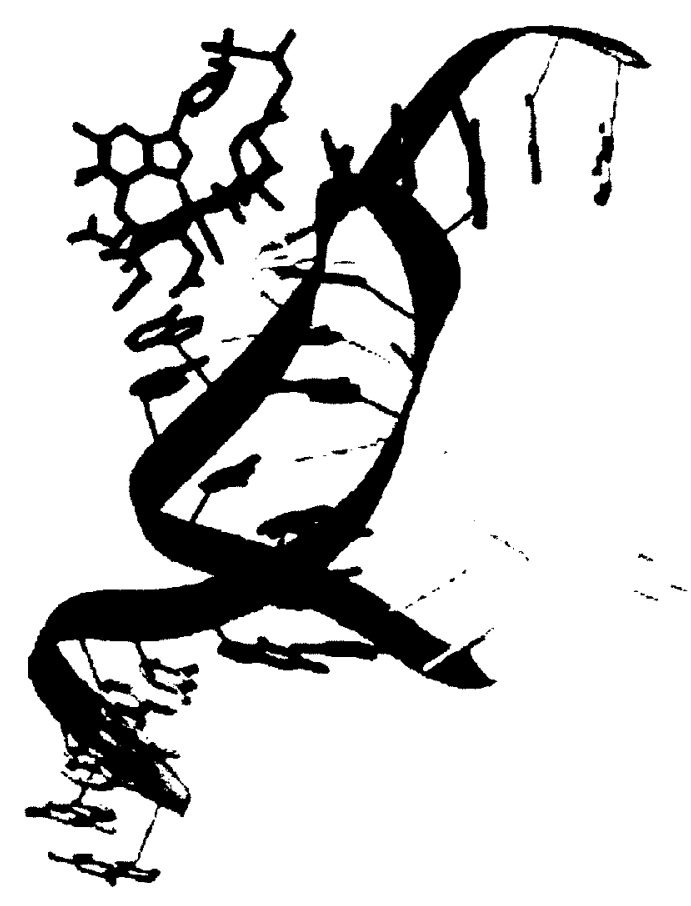

Figure 1.6: Aptamer forming secondary structures upon binding to its target, cyanocobalamin ${ }^{54}$. The secondary structures in this situation include a three-stranded zipper and a pair of three-nucleotide loops.

As biological sensing molecules, aptamers are always contrasted against antibodies.

Aptamers have some clear advantages over the use of antibodies. Firstly, aptamers are selected in vitro compared to in vivo for antibodies which allows for more control and minimized batch-to-batch variation ${ }^{55}$. Second, high-throughput methodologies have resulted in shorter time for generation of aptamers relative to antibodies ${ }^{56}$. Moreover, since aptamers are synthesized in the laboratory, it is relatively easy to modify the strands with functional groups or fluorophores ${ }^{57}$. This allows for the further use of 
aptamers as biosensors, which is a field very actively researched. Aptamers also have high discrimination power between very similar molecules. Polisky and co-workers ${ }^{58}$ found that an aptamer generated for theophylline (a therapeutic) can differentiate by a factor of 10,000 between caffeine, which differs from theophylline only by a single methyl group. Polisky found that their theophylline aptamer was able to discriminate between theophylline and caffeine better than a protein antibody developed for the same target ${ }^{59}$. Due to their relative ease of generation compared to antibodies, aptamers have been manipulated for a variety of biological applications, including therapeutics and diagnostics. Currently, there is one FDA approved aptamer-based therapeutic for Macular Degeneration (MacuGen) since $1995^{60}$, and additional eight in clinical trials for different biological targets ${ }^{61}$. Aptamers have been developed for a variety of therapeutic targets, including $\alpha$-thrombin, HIV-reverse transcriptase, fibroblast growth factor 2 , respiratory syncytial virus, HIV-1 integrase, vascular endothelial growth factor, platelet-derived growth factor, immunoglobulin E, L, D and P selectin, interferon- $\gamma, \mathrm{D}$-adenosine, acetylcholine auto-antibodies, keratinocyte growth factor, neutrophil elastase, phospholipase $A_{2}$, protein tyrosine phosphotase, activated protein $\mathrm{C}, \mathrm{CD} 4$, nuclear factor-kB, lymphocyte function-associated antigen 1 , cytohesin 1 , and av $\beta 3$ integrin ${ }^{61 .}$ More recently, cancer cell targeting has been shown using an aptamer which subsequently released a prodrug from a drug-loaded nanoparticle ${ }^{62}$. Aptamers are selected in vitro through the Systematic Evolution of Ligands by EXponential enrichment (SELEX). Figure 1.7 shows a typical SELEX experiment. In SELEX, 
a random pool of ssDNA (or RNA) is synthesized using laboratory scale synthesizers. Typically, these pools result in $\geq 10^{15}$ molecules, with the hypothesis being that one (or some) of those sequences will attain the correct nanoscale conformation to bind to the target molecule ${ }^{63}$. The molecules are then selected (through elution) against the target, which in the case of small molecules is typically tethered to beads on a column. After elution, the unbound sequences are discarded, and the bound sequences collected through competitive elution using the target in solution and sometimes a denaturing agent (e.g., Urea). The potential aptamers are amplified through the Polymerase Chain Reaction (PCR), which enriches the pool with occasional random mutations creating a mutated pool which may have some stronger binders relative to the unenriched parent pool. The cycle of selection is repeated $n$ times. A counter selection can also be incorporated into SELEX which will remove strands with low discrimination efficiency. Finally, cloning and sequencing the final strands will allow for characterization of the aptamers and subsequent binding studies can indicate the best aptamer candidate for the given target. 


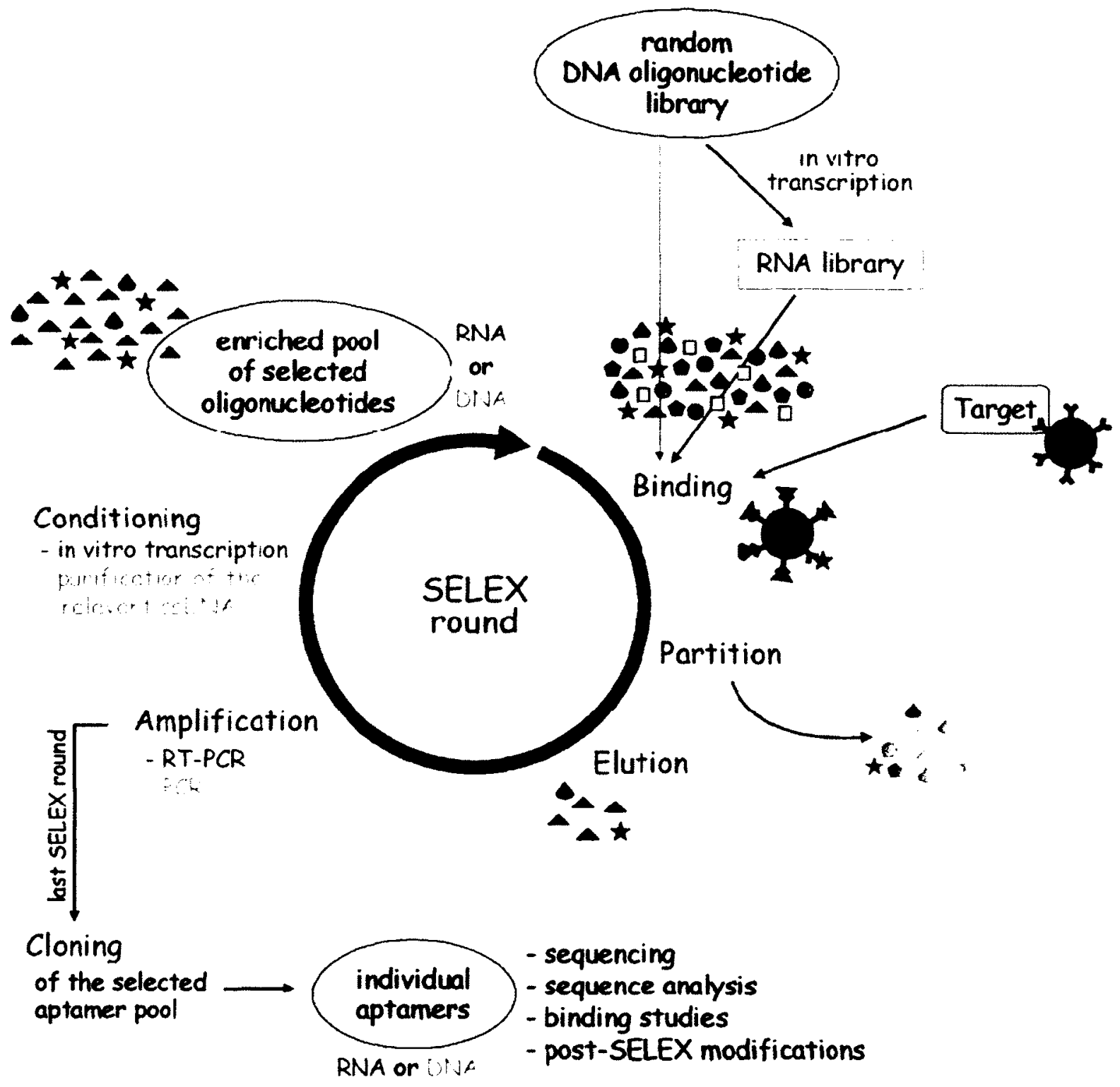

Figure 1.7: Overview of Systematic Evolution of Ligands by EXponential enrichment (SELEX). A random pool (library) of sSDNA or RNA strands are selected against a target, typically bound to a column or through high throughput methodologies. Unbound DNA/RNA molecules are discarded while bound molecules are collected, typically through competitive elution. The potential candidates are then enriched through PCR. The cycle is then repeated $n$ times, typically with increasing stringency each round. The final strands are then cloned and sequenced, and commonalities amongst the strands sequences can give an indication on the secondary structure formed through computational modeling (e.g., q-quadruplex). Binding affinity studies are done on the strands to indicate the best binding 'aptamer ${ }^{64}$. 
Since 1990, the process of SELEX has remained largely unchanged with very minor modifications. Most changes to SELEX have focused on reducing the number of selection cycles (i.e., cost and time) for aptamer generation. For example, single-round SELEX has been used with non-equilibrium capillary electrophoresis for the generation of highaffinity aptamers. This methodology allows for higher efficiency of detecting aptamertarget binders (relative to using column elution) and determination of binding affinity constants $\left(K_{d}\right)$, all in a single round of SELEX ${ }^{65}$. Other groups have looked at methodologies to improve detection sensitivity for $K_{d}$ determinations using optics ${ }^{66}$ and resonators ${ }^{67}$.

In terms of aptamer binding, a few trends have emerged over the past twenty years. First, tighter binding (lower $K_{d}$ ) means higher specificity. This is due to the higher number of $\mathrm{H}$-bond donor and acceptor networks formed ${ }^{68}$. Second, large molecules are bound better than smaller molecules, likely because of more associative electrostatic interactions. Third, targets with heterocyclic bases bind better to aptamers without, which is why it is easier to find aptamers for targets containing such moieties ${ }^{68}$. Easy strand modification coupled with the ability to monitor the shape change of the aptamer strand has made them attractive as biosensors. Aptamers can be used for (1) optical sensing where a signal such as fluorescence can indicate changes upon aptamertarget binding, (2) acoustic sensing by depositing an aptamer or target on a resonator (such as quartz crystal microbalance) and detecting frequency (mass) changes, (3) cantilever based sensing by modifying the tips of atomic force microscopes and 
studying the interaction between the tip and modified sample surface (by aptamer or target); and (4) electrochemical sensing to detect target analytes on modified electrodes or electrochemical cells ${ }^{69}$. Aptamers have been used as biosensors to detect many analytes including cocaine (fluorescent) ${ }^{70}$, thrombin (electrochemical) ${ }^{71}$, VEGF and other cancer associated proteins (fluorescence) ${ }^{72}$, listeria monocytogenes (optical) ${ }^{73}$, and adenosine (optical) ${ }^{74}$. Figure 1.8 below shows a generic scheme for employing aptamers in various types of biosensors. 


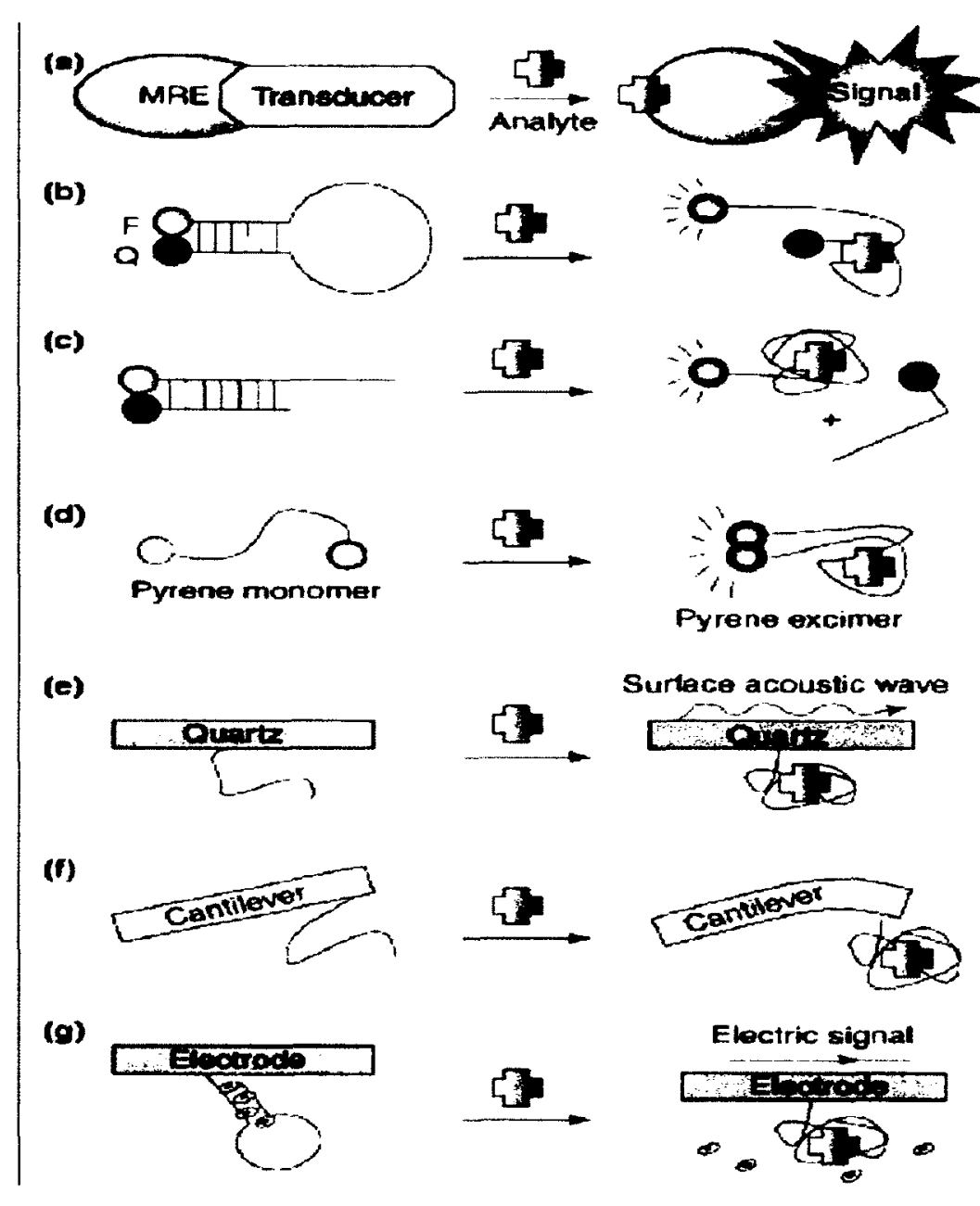

Figure 1.8: Types of aptamer biosensors: (a) generic scheme showing detectable signal production upon binding of a molecular recognition element (MRE; e.g., aptamer) and analyte (e.g., target).; (b) an aptamer in its native form is quenched due to fluorophorequencher pair spatial arrangement, while upon binding to the aptamer the pair is spatially far from each other and quenching cannot occur and a resultant signal is seen; (c) an aptamer in its unbound form is complexed with its antisense counterpart which contains a quencher to quench the fluorophore on the aptamer. Upon target presence, the antisense strand is removed due to increased complimentarity and as the quencher leaves, fluorescence is re-established and can be detected; (d) modifying an aptamer on both ends with a species able to form excimers (such as pyrene), upon target binding the close spatial arrangement allows for excimer formation and subsequent specific excimer fluorescence at a longer wavelength; (e) mass-based sensor showing the change in mass (frequency of an acoustic wave) upon mass addition of the target (used in quartz crystal microbalance); (e) cantilever biosensor with the aptamer modified at the tip and change in deflection can be detected as the aptamer binds the target present on the sample surface; and (f) electrochemical sensors where change in current upon aptamer target binding can be measured ${ }^{75}$. 
As already highlighted, much research has focused on the discovery and development of aptamers whilst little has been driven towards incorporation of the aptamers into polymer matrices. Only over the past 2-3 years papers looking at aptamers in polymer films have emerged. Even more scarce are papers dealing with aptamers in polyelectrolyte multilayers. Over the past 3 years, only 4 papers have been published looking at aptamers in films, 3 of which have been dealt with polyelectrolyte deposition of polymers with aptamers.

In an effort to detect pathogens on paper surfaces, Su et. al. ${ }^{76}$ investigated the binding of ATP on aptamer functionalized cellulose. They found $25 \%$ coupling efficiency of the modified aptamer to cellulose, without the need for spacers, led to a highly specific and reversible binding of ATP to the film surface. Du et. al. ${ }^{77}$ have deposited aptamers on polyelectrolyte films to electrochemically detect thrombin and lysozymes. In their system, aptamers were deposited as the topmost layer of the polyelectrolyte multilayers. They have shown that upon aptamer-target binding, a barrier for electrons is formed which inhibits electron transfer resulting in decreased differential pulse voltammetry (DPV) signal of their electroactive probe (ferrocene). Zhang et. al. ${ }^{78}$ have recently developed a photoelectrochemical cytosensor using polyelectrolyte multilayers of aptamer modified CdSe quantum dots (anionic) and poly(diallyldimethylammonium chloride) (cationic). They found that their sensor exhibited high sensitivity and specificity to Ramos cells (target). Their biosensor established a linear range of $160-1600$ cells $/ \mathrm{mL}$ and a detection limit of 84 cells $/ \mathrm{mL}$. Similarly, Yuan et. al. ${ }^{79}$ have developed a label-free 
electrochemical polyelectrolyte multilayer sensor using thrombin aptamer modified (via thiol) citrate capped gold nanoparticles (anionic) and nafion (cationic) using a layer-bylayer self-assembly. In their system, the aptamers were placed on the surface of the film. They found high sensitivity and linearity from $0.12 \mathrm{nM}$ to $46 \mathrm{nM}$ with a detection limit of 40pM.

All the above mentioned studies involve deposition of aptamers on film surfaces and measuring a signal (as biosensors) to improve sensitivity or detection limits. Other than the papers published from this thesis, there has been no work done on embedding aptamer within porous polyelectrolyte films. Embedding aptamers in such films, effectively protecting the aptamer from the surrounding environment, can be quite advantageous for in vivo applications. For example, aptamers have been found to suffer the same challenges as other therapeutics and pharmaceutics in terms of rapid renal clearance in the body. In an effort to improve bioavailability, groups have tethered aptamers to bulky groups such as cholesterol and poly(ethylene glycol) ${ }^{80}$. This has increased the bioavailability of aptamers, however, the aptamer is still in contact with the plasma environment and as such can undergo other types of conjugation from defensive mechanisms and/or degrade. By embedding the aptamer in polyelectrolyte multilayers, one can effectively tailor the bioavailability of the system while protecting the aptamer from non-specific conjugation and degradation, and thereby, increasing its function. It is understood that these processes cannot completely be mitigated, however, they can be reduced to increase the aptamers usefulness and functionality. 
This methodology can also be applied to our smart fertilizer concept, where the aptamer embedded in the film can be protected from the outside environment and continue to function and bind to its target as it permeates through porous films.

Aptamers are always characterized in terms of their binding affinity. The concept of binding affinity is introduced in the following section and is used throughout this thesis to show specificity and sensitivity of our aptamers, aptamer embedded PEMs and nanoaggregates.

\subsubsection{Binding Affinity}

According to the law of mass action, 'the velocity of a chemical reaction is proportional to the product of reactant concentration(s)'. Considering one kind of reactant molecule (A) being transformed to $B$, the reaction rate can be described by: ${ }^{81}$

\section{$A \rightarrow B$}

$$
\frac{d A}{d t}=-\frac{d B}{d t}=k[A]
$$

In the case of 2 reactant molecules combining to form one target product, the rate is:

$$
\begin{aligned}
& \mathrm{A}+\mathrm{B} \rightarrow \mathrm{C} \\
& \frac{d C}{d t}=-\frac{d A}{d t}=-\frac{d B}{d t}=k[A][B]
\end{aligned}
$$


The law of mass action assumes: (1) all aptamers are equally available for target binding; (2) aptamers are either bound $\left(K_{o n}\right)$ or unbound $\left(K_{o f f}\right)$ to the target, and there is no other state; (3) binding does not change the aptamer or target permanently; and (4) the binding is reversible.

Extrapolating from the law of mass action, aptamer-target binding in a reversible system ( $K_{\text {on }}$ and $\left.K_{\text {off }}\right)$ can be described by:

In the forward direction: Aptamer + Target $\stackrel{K_{\text {on }}}{\rightarrow}$ Aptamer-Target In the reverse direction: Aptamer-Target $\stackrel{K_{\text {off }}}{\rightarrow}$ Aptamer + Target

For the forward direction, the rate of association is: $k_{\text {on }}{ }^{*}$ [Aptamer][Target]

For the reverse direction, the rate of dissociation is : $k_{\text {off }}$ [ [Aptamer*Target]

Forward direction reactions occur when the aptamer and target collide due to Brownian motion in a random state, so the rate is proportional to the concentration of each species, i.e., increases the probability of collision. While in the reverse direction, the dissociation is a random process, such that at each time point the probability of dissociation is the same, thus the dissociation is dependant only on the concentration of the bound ligand-receptor complexes. At equilibrium, the binding affinity constant $\left(K_{d}\right)$ can be calculated as the ratio between the reactions in the forward and reverse direction: 


$$
K_{\mathrm{d}}=\frac{K_{\mathrm{off}}}{K_{\mathrm{on}}}=\frac{[\text { Aptamer }][\text { Target }]}{[\text { Aptamer } \cdot \text { Target }]}
$$

The $K_{d}$ is defined by the concentration at equilibrium where half of the target molecules are bound to the aptamer. So if the aptamer has a high affinity for the target, even at low concentrations there will be a very low $K_{d}$ (good binding affinity), while if the affinity is weak, high concentration of the aptamer and target are required for half of the target to be bound to the aptamer and thus the $K_{d}$ will be high (weak binding affinity). Thus, a relative strength of binding affinity can be measured by evaluating $K_{d}$ 's. A more useful equation which is commonly used to evaluate $K_{d}$ 's allows for the determination of the fraction of all aptamers bound to a target since it is not always possible to ensure that all the aptamers are bound to the target:

$$
\text { Fraction Occupancy }=\frac{[\text { Target }]}{\left[\text { Target }+K_{\mathrm{d}}\right]}
$$

Equation 4 can be used for $K_{d}$ evaluation when there is only specific binding of the aptamer-target complexes. In the case where there is a specific and non-specific interaction and assuming that (1) both binding events have their own respective receptors $\left(B_{\max }\right)$; and (2) the two binding events are unrelated, $K_{d 1}$ (specific) and $K_{d 2}$ (non-specific) can be determined by:

$$
\frac{B_{\max 1} \cdot[\text { Target }]}{\left[K_{\mathrm{d} 1}+\text { Target }\right]}+\frac{B_{\max 2} \cdot[\text { Target }]}{\left[K_{\mathrm{d} 2}+\text { Target }\right]}
$$


Assuming infinite binding sites, Equation 5 becomes a geometric expansion (series) of Equation 4. Thus, one can evaluate multiple binding events in a single system.

\subsection{Thesis Objectives}

The objective of this thesis is to explore feasibility (in vitro) of a smart fertilizer system. Eventually, this system will be based on PEMs containing an aptamer specific to a plant exudate specific to growth and nutrient needs. The fertilizer will be released selectively upon aptamer-target binding whilst the PEMs will protect the aptamer from premature degradation and non-specific interactions from the soil environment. In this thesis, we will examine model systems to determine the feasibility of such a system. We chose an aptamer for a dye (sulforhodamine B) thereby allowing easy detection using absorbance and fluorescence. Towards this goal, the following chapters demonstrate that aptamers can retain their binding affinities with high selectivity and sensitivity in bilayer films (Chapter 2), the diffusion of the target (Chapter 3) and small molecules (Chapter 4) through the walls of PEM microcapsules can be altered upon aptamer-target binding. Also discussed are diffusion studies utilizing an aptamer for a possible plant exudate Iysine (Chapter 5). Chapter 6 explores the idea of aptamer superstructures and possible tailoring of binding affinities for the same target based on operational requirements, such as requiring ultrasensitive detection of plant exudates for targeting of the smart fertilizer. It is clear that our findings can be extrapolated to other uses such as drug delivery, therapeutics and diagnostics. 
This fertilizer system will be used in the environment and as such will require thorough investigation into the fate and effects before being introduced into field-scale applications. While it is outside the scope of this thesis to try and elucidate some of these transformations, we should be mindful of the potential impacts these materials may have. Dealing with these challenges through involvement in international and domestic activities, such as those within the OECD, is the primary way to ensure timely outputs while maximizing Canadian leverage and impact while minimizing duplication of efforts. We feel that our nanoparticle system has the potential to greatly impact the global agricultural and environmental landscape and such will require understanding of any future impacts. 


\section{Chapter 2. Bilaver Films}

Sultan, Y.; Walsh, R.; Monreal, C.; DeRosa, M. Biomacromolecules, 2009, 10(5), 1149 -

1154.

\subsection{Introduction}

\subsubsection{Aptamer bilayer films}

The potential for the use of aptamers as molecular recognition elements is clear, but in order to fully exploit these nucleic acids for the fabrication of biosensors and the development of other applications, aptamers will need to retain their functionality when embedded in or attached to a suitable matrix. Aptamers have been immobilized in a sol-gel ${ }^{82}$, tethered to cellulose ${ }^{83}$, and attached to a range of surfaces ${ }^{84}$ all without loss of their binding ability. Yet despite the great promise of $\mathrm{lbl}$ polyelectrolyte films for a wide range of applications, no work on the entrapment of aptamers within these films has been reported. The objective of this work was to determine whether the Ibl approach could be used to prepare a robust aptamer film that maintained its affinity and specificity for the cognate target.

Here, we report on the first successful incorporation of a DNA aptamer into a multilayered polyelectrolyte thin film (Figure 1). We chose the sulforhodamine B (SB) aptamer $^{85}$ as our model system for this study. UV-Vis spectroscopy, Quartz Crystal Microbalance (QCM) studies, confocal microscopy, and Time of Flight Secondary lon Mass Spectrometry (ToF-SIMS) all confirmed that the aptamer retained its recognition ability despite being embedded in the bilayer film. These films were simple to prepare 
and were stable and reusable. This opens up new possibilities for the development of aptamer-based functional films for use in biosensing, implant coatings, controlledrelease and many other applications.

\subsubsection{Quartz Crystal Microbalance}

The Quartz Crystal Microbalance (QCM) is driven by the concept of the piezoelectric effect. The piezoelectric effect was discovered in 1880 by Jacques and Pierre Curie who discovered that applying mechanical stress to certain materials resulted in an electrical potential which had a magnitude proportional to applied stress. The Curies were able to prove the converse situation as well, where applying an electrical potential resulted in a mechanical strain of the crystal. This converse situation drives the theory of QCM

(Figure 2.1). They found the piezoelectric effect to be unique to acentric crystals, i.e., molecules which contain a dipole as a result of atom orientation in the lattice (noncentrosymmetric space groups). Typical examples of such crystals include quartz, rochelle salt, and tourmaline ${ }^{86}$.


Figure 2.1: A typical: (a) AT-cut quartz crystal; and (b) sheering as a result of applied electrical potential. In the case of quartz, this sheering is reversible ${ }^{86 .}$ 
Typically in QCM, a quartz crystal cut by slicing a silica rod at a ca. $35^{\circ}$ angle relative to the $\mathrm{x}$-axis (AT-cut crystal) is used. As can be seen from Figure 2.1(b), applying an electric field across the crystal will result in a shear strain proportional to the applied field. In the case of quartz, this is reversible, i.e., applying an equal electric field in the opposite direction will allow the crystal to shear in the opposite direction. Thus, by oscillating the crystal through the application of sequential opposite electrical fields, a transverse acoustic wave can be generated which spans the thickness of the crystal at very high frequencies. The frequency $\left(f_{o}\right)$ of this standing wave can be calculated by ${ }^{86:}$

$f_{\mathrm{o}}=\frac{v_{\mathrm{tr}}}{2 t_{\mathrm{q}}}=\left(\mu_{\mathrm{q}}^{1 / 2} / \rho_{\mathrm{q}}{ }^{1 / 2}\right) / 2 t_{\mathrm{q}}$

Where $v_{t r}=$ transverse velocity of sound of sound in the AT-cut quartz crystal $\left(3.34 \times 10^{4}\right.$ $\mathrm{m} / \mathrm{s}) ; \mathrm{t}_{\mathrm{q}}=$ thickness of the crystal; $\mu_{\mathrm{q}}=$ sheer modulus of the quartz; and $\rho_{\mathrm{q}}=$ density of the quartz.

If a foreign material (such as a film) is deposited on the quartz surface, and assuming that the foreign material has the same properties (e.g., refractive index) as the quartz crystal, the added film, in turn, becomes just an extension of the quartz crystal itself, changing the frequency of the standing wave as the thickness of the overall crystal changes. Assuming an incremental change in the thickness $(\Delta t)$ will result in an incremental change in the frequency $(\Delta f)$, Equation 1 then becomes ${ }^{86}$ : 


$$
\frac{\Delta f}{f_{\mathrm{o}}}=-\frac{\Delta t}{t_{\mathrm{a}}}=-2 f_{\mathrm{o}} \Delta t / v_{\mathrm{tr}}
$$

Combining equation (1) and (2) and expressing $\Delta t$ in terms of change of mass $(\Delta \mathrm{m})$ and areal density $(A)$, the well-known Sauerbrey equation can be derived ${ }^{86}$ :

$$
\Delta f=-2 f_{\mathrm{o}}^{2} \Delta m / A\left(\rho_{\mathrm{q}} \mu_{\mathrm{q}}\right)^{1 / 2}
$$

From the Sauerbrey equation, one can calculate the change in mass from a change in frequency (i.e., vibration of the crystal as the added mass of a foreign material changes the shear rate of the quartz). QCM works well for thin films on the nanoscale, since the assumption that the added film is the same as the quartz crystal (same density) holds true as long as it is thin relative to the quartz. As the film gets thicker (on the micron scale), the equation does not accurately reflect mass changes, but rather becomes the combination of two frequencies, one from the quartz and the second of the foreign material on the surface ${ }^{86}$. In such cases, Lu and Lewis have developed an expanded Sauerbrey equation which incorporates the density and shear modulus of the foreign film. This equation is not discussed here as we are dealing with thin (nm scale) films, but can be found in the cited reference ${ }^{87}$. Modifications in the Sauerbrey equation have also made it possible for QCM to be used in liquid samples ${ }^{88}$. QCM has also been actively used to study the dynamics of films ${ }^{89}$ through Dissipation (QCM-D), where the crystal, 
once at its resonance frequency, is disconnected and allowed to decay exponentially and the frequency change between the expected and measured decay calculated. A typical QCM AT-cut quartz crystal is shown in Figure 2.2 below. The crystal is modified with an electrode at each face (top and bottom), typically gold, to produce electric potential.

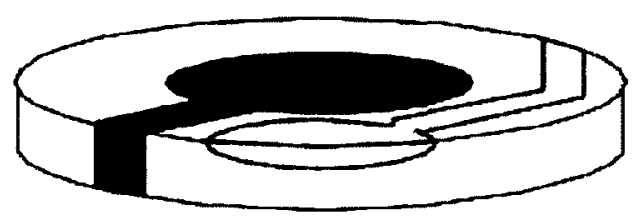

Figure 2.2: A typical QCM quartz crystal showing two electrodes (gold and grey) deposited on each face of the quartz (white) ${ }^{90}$.

Figure 2.3 shows a Butterworth Van Dyke electrical circuit, which is used to build QCM's.

The circuit consists of two arms. The motional arm detects change in mass and viscous loading of a sample where $R$ (resistor) is the dissipation in oscillation energy as a result of a medium coming in contact with the crystal, $C_{s}$ (capacitor) is the stored energy of the oscillation and is related to the elasticity of the crystal, $L$ (inductor) is the inertial component of the arm which is related to the change in mass by measuring vibration (change in frequency). $C_{p}$ is the parasitic capacitance which is the sum of capacitances from the crystal electronics and equipment (such as holder, electrodes, etc). To measure quantitative data, this value needs to be cancelled from the display controls on the QCM instrument by the addition of a feedback loop which is standard in all $\mathrm{OCM}^{\prime} \mathrm{s}^{91}$. 


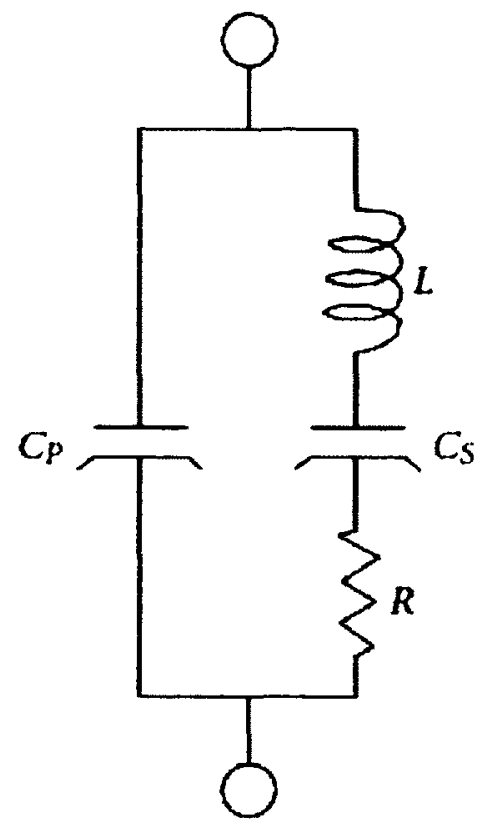

Figure 2.3: Butterworth Van Dyke electrical model showing the motional arm with inductor (L), Resistor (R), Capacitor (Cs), and parasitic Capacitance $(C p)^{92}$.

\subsubsection{Time-of-Flight Secondary Ion Mass Spectrometry (ToF-SIMS)}

Mass Spectrometry (MS) allows for the measurement of mass of charged molecules to very high accuracy (mass/charge ratios). Extensive reviews on MS can be found in works from Vestal ${ }^{93}$, Douglas ${ }^{94}$, Douglas ${ }^{95}$, Fenn ${ }^{96}$, Mann $^{97}$, and March $^{98}$. A thorough review on Time of Flight (TOF) can be found in Wollnik ${ }^{99}$.

Secondary Ion Mass Spec (SIMS) is based on the principle that ions or charged molecules are ejected from a solid material (e.g., films) upon bombardment. SIMS was first used by Herzog and Viehboeck, who also made the first ion source in $1949^{100}$. During bombardment, excited surface species leave the sample surface and enter the gas 
phase. These excited surface molecules may subsequently degrade or fragment into smaller species (into secondary ions) which can be separated according to their mass/charge $(\mathrm{m} / \mathrm{z})$ ratio. The sample may be sputtered off at low sputter rates, i.e., gently bombarding the sample surface with excited molecules and causing the surface to fragment into the gas phase (static SIMS). Alternatively, the sample may 'lifted-off' at high sputter rates which provides a depth profile of the sample material (Figure 2.4) ${ }^{100}$.

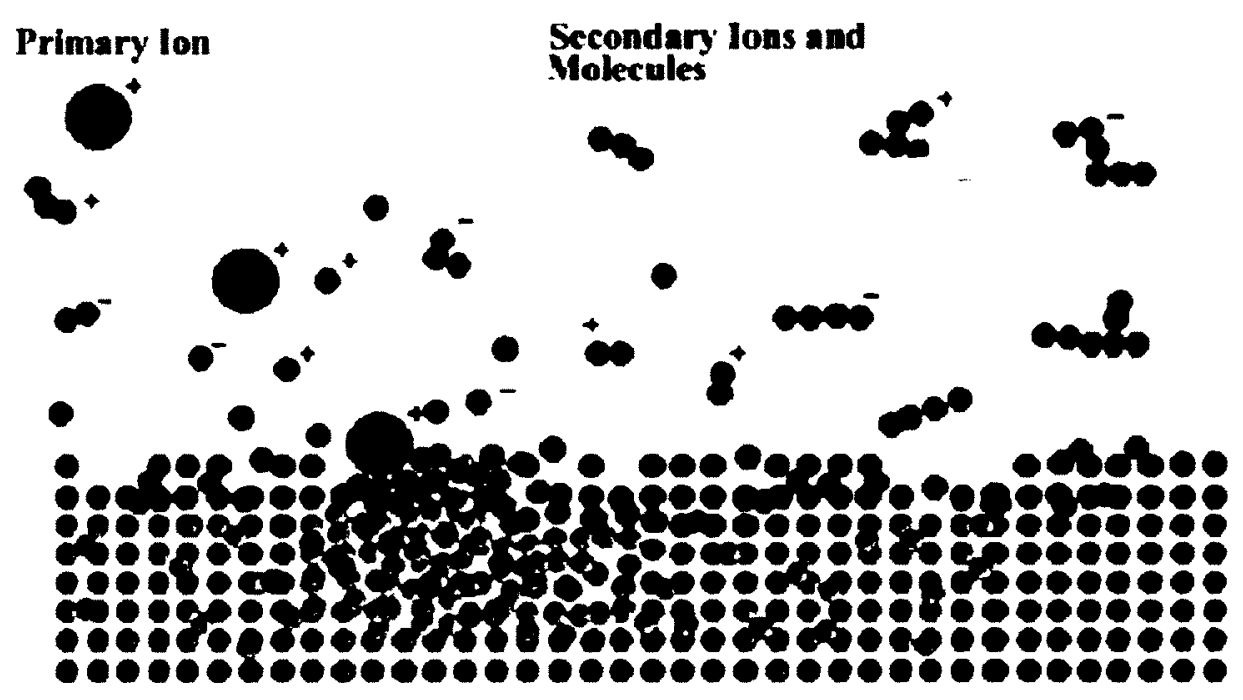

Figure 2.4: A sample surface being bombarded with primary ions causing the surface to fracture/deform and release secondary ions into the gas phase. The secondary ions may be charged or neutral and may fragment once in the gas phase. Post-bombardment bombardment in the gas phase can excite neutral fragments ${ }^{101}$.

ToF-SIMS instrumentation requires three elements: first, a source must provide the primary particles for bombardment on the sample surface (both in static and dynamic modes), second, the secondary ions need to be separated according to $\mathrm{m} / \mathrm{z}$ using ToF and third, the ions need to be detected in a MS. The primary particles used are typically 
positively charged $\left(\mathrm{Ar}^{+}, \mathrm{Xe}^{+}, \mathrm{Ga}^{+}\right)$with negatively charged $\mathrm{O}_{2}^{-}$used on occasion. A typical ToF-SIMS instrument is shown below ${ }^{102}$ :

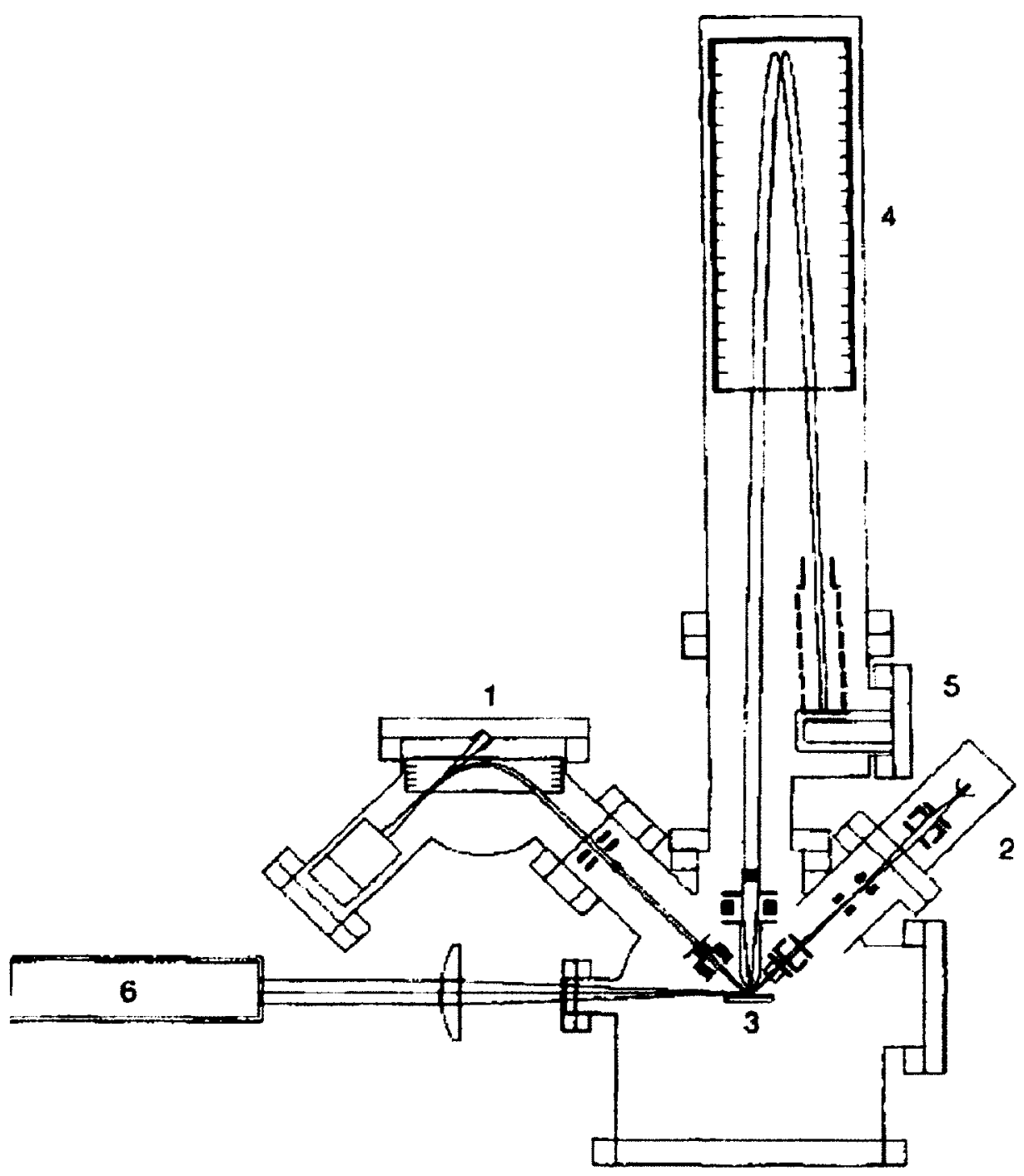

Figure 2.5: A typical high resolution ToF-SIMS instrument showing a primary ion source (1) to bombard the sample surface, an electron impact source (2) used in traditional ToF-MS, the target (3), reflectron in the ToF tube (4), and the MS detector (5). In this ToF-SIMS an optional post-ionization ion source is also added $(6)$ to ionize neutral fragments in the gas phase. ${ }^{103}$ 
Once excited, the secondary ions can be either neutral or charged in the gas phase.

Subsequently, as they enter the ToF, they can further fragment into charged or neutral species and/or charged ions can be converted to neutral species or vice versa. Only the charged species are detected by the MS. Post-ionization lasers can be used to ionize neutral molecules exiting the surface into the Tof tube ${ }^{103}$. The polarity of the electric field can be selected by the user, and as such both positively and negatively charged ions can be encouraged to enter the MS detector. The velocity of the secondary ions in the ToF is directly related to the $\mathrm{m} / \mathrm{z}$ ratio as the ions pass a constant potential difference $(U)$ according to:

$E_{\mathrm{kin}}=\frac{m}{2} \cdot v^{2}=\frac{m}{2} \cdot \frac{s^{2}}{\Delta t^{2}}$

$E_{\text {kin }}=\mathbf{z} \cdot \boldsymbol{e}_{0} \cdot \boldsymbol{U}$

Where; $E_{k i n}=$ kinetic energy consumed by the charge; $z=$ charge; $e_{o}=$ elementary charge; $v$ = velocity; $s$ = distance (equal for all ions); $\Delta t=$ time interval, defined by the time of primary particle injection on the sample surface (start) and the excited ions entering the detector (end).

Once in the flight tube, the ions are reflected through reflectron electronics and focused on to the detector. Thus, according to Equation 4, one can measure the abundance of specific species (according to $\mathrm{m} / \mathrm{z}$ ) on the sample surface (static) or as a depth profile (dynamic). For example, in this project dynamic ToF-SIMS was employed, using positive 
primary particles to sputter the films. These secondary ions were then separated according to $\mathrm{m} / \mathrm{z}$ and their relative abundance as a function of depth was measured. This allows for the tracking of specific ions as a depth profile ${ }^{104}$. By mapping the distribution of ions over a surface, high resolution images can be developed. Different optimizations can be made to ensure maximum output of the ToF-SIMS. It can be optimized to maximize mass resolution, i.e., bunched mode where the ions are collected over short bursts and then analyzed, or maximize spatial resolution, i.e., burst alignment mode where quick scans without adding time for ion bunching increase resolution but not nominal mass determination. If maximum spatial resolution or mass determination is not required, burst mode can be used, where the primary beam repeatedly bombards the surface to increase ion current and thus give a good measurement on the mass and spatial resolution ${ }^{105}$. Figure 2.6 shows the different modes. Due to the versatility of ToFSIMS and the fact that one can do quantitative analysis on the chemical species in a solid sample, it is used extensively to study different synthetic and biological surfaces ${ }^{106}$. 

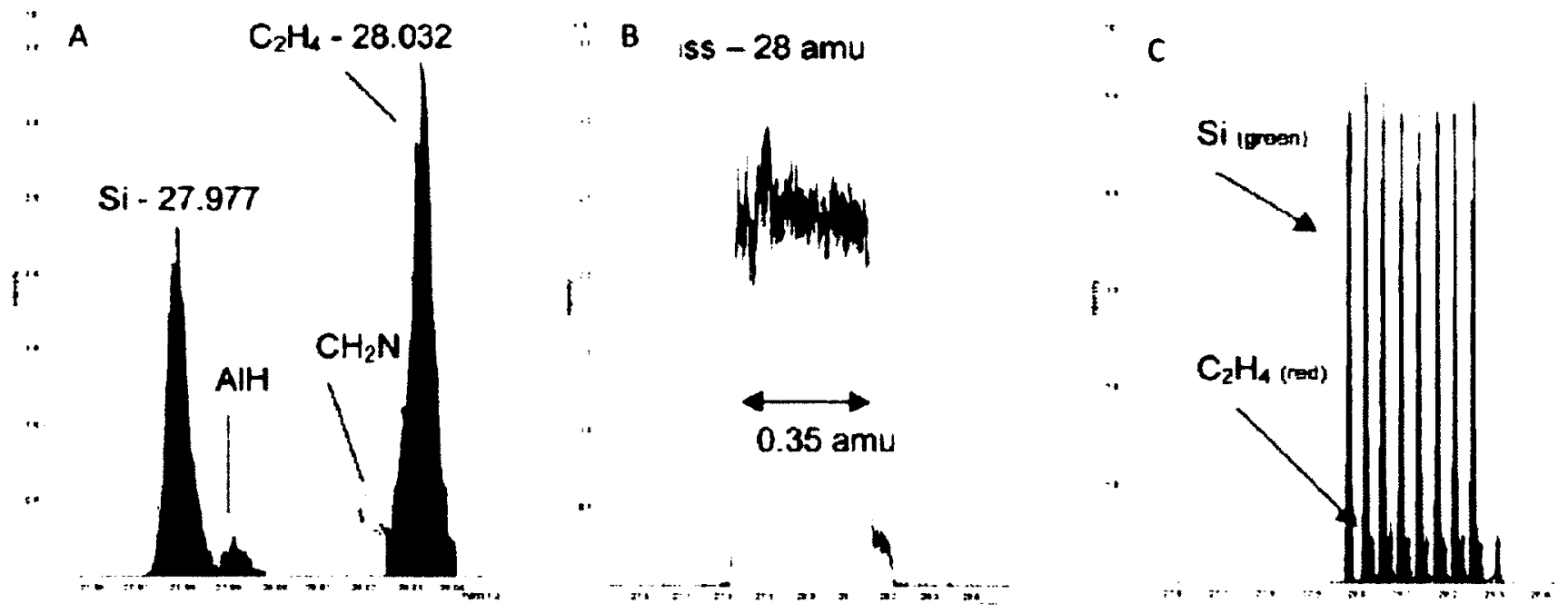

Figure 2.6: Example of different modes of ToF-SIMS depending on operational needs: (a) bunch mode showing high mass resolution; (b) burst alignment showing high spatial resolution; and $(c)$ burst mode, used when information on both mass and spatial resolution is needed without maximum resolution of each ${ }^{107}$.

\subsubsection{UV/Vis Spectrophotometry}

Molecules absorb characteristic wavelengths of light depending on their chemical environments. In absorbance spectroscopy, light (ultraviolet or visible) is split by a prism into different wavelengths. These wavelengths of light interact with the molecules (incident light) in the sample and certain wavelengths are absorbed depending on the energy and allowedness of electronic transitions within the molecules. . The absorbed wavelengths can give an indication of the type of molecules present in the sample. A diffraction grating is used to select specific wavelengths to interact with the sample. In absorbance spectroscopy, transitions from the ground state to the excited state are probed; this is in contrast to fluorescence where the emission of the light is measured as electrons return from excited states to ground states (See Chapter 5). When absorbance 
spectroscopy is coupled with measuring the amount of light absorbed or transmitted by the sample, it is termed spectrophotometry. A typical single beam spectrophotometer is shown below in Figure 2.7.

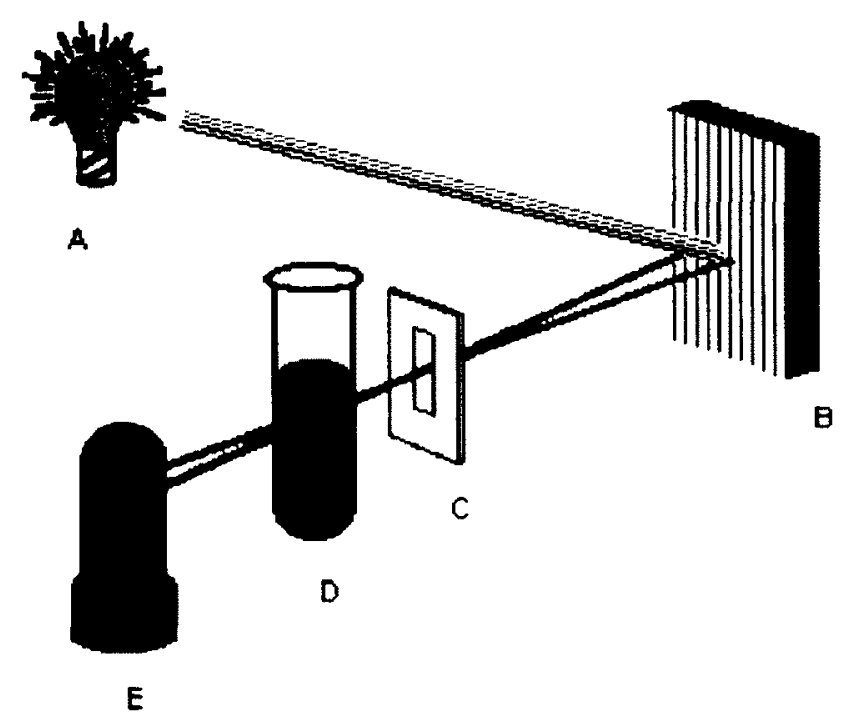

Figure 2.7: Typical single beam UV/vis spectrophotometer showing the light source (A), the grating which selects specific wavelengths for the sample $(B)$, aperture $(C)$, sample (D), and detector (E).

The absorbance and transmittance of the sample can be calculated from the equations of Heinrich Lambert (1728-1777) who demonstrated (Lambert Law) that the proportion of light absorbed by a substance is independent of the intensity of incoming radiation (incident light).

$$
A=\log \left(\frac{I_{0}}{I}\right) \quad T=I / I_{\circ}
$$

Where; $A=$ absorbance, $I=$ transmitted light, $I_{0}=$ incident light,$T=$ transmittance 
The percentage transmittance is then just the transmittance multiplied by 100 . Wilhelm Beer (1797-1850) demonstrated (Beer Law) that the Absorbance (Equation 5) is simply a constant $(\varepsilon)$ times concentration $(c)$. Thus, combining the two equations, the BeerLambert Law can be derived which relates the Transmittance (ratio between the transmittance and incident light) to the concentration of the sample. As can be seen the absorbance is proportional to the concentration of the molecules.

$$
A=\log \left(\frac{I_{0}}{I}\right)=\varepsilon c l
$$

Where; $\varepsilon=$ absorption coefficient (or extinction coefficient),$c=$ concentration, and $\mathrm{I}=$ light path through the sample.

Thus, using the Beer-Lambert law, molecules can be quantified and additional studies such as kinetics of molecular change and binding affinities $\left(K_{d}\right)$ can be performed.

\subsubsection{Atomic Force Microscopy (AFM)}

Atomic Force Microscopy (AFM) was first envisioned by Gerd Binnig in 1985. As suggestive from the name, in AFM the force or change in force is measured as a tip interacts with a modified surface ${ }^{108}$. These deflections can be a result of physical stress and/or covalent interactions, or long-range disruptions such as magnetic fields. In conventional AFM, a tip can either be in physical contact with the surface (contact 
mode) or interact through non-contact forces with a surface to generate surface images on the nanoscale. Due to the use of piezoelectric materials for the stage and/or tip holders along with having very sharp tips on the nanoscale, AFM can image at low size scales (sub nanometer scale) with high resolution. Due to its versatility and relatively low cost compared with electron microscopes, it is used routinely in materials science. AFM also have many advantages in the field of biomaterials as the sample does not undergo degradation or permutations as a result of electron beam bombardment, which is the case with electron microscopes, thus preserving very sensitive biological surface structures $^{109}$

\subsubsection{Laser Scanning Confocal Microscopy (LSCM or CLSM)}

The fundamentals of light microscopy were originally developed by Ernst Abbe in 1873 and 1884 . He described how an image can be generated from measuring the diffraction of light as it interacts with a sample surface. In a traditional light microscope, a coherent wave (in phase) interacts with a sample, and depending on this interaction, the transmitted wave shifts relative to the incoming wave (incoherence) (Figure 2.8(a)). Depending on the degree of incoherency, an image of the substance can be formed. The image is a graphical representation of the relative incoherence of light generated over a 2-dimensional space. Traditionally, microscopes contain an objective lens, which collects the transmitted light from the specimen and a condensing lens, which focuses the light onto the specimen (Figure $2.8(b)$ ). In contrast to Figure $2.8(b)$, microscopes can also be 
inverted, where the light source and condenser lens are on top and the objective lens is at the bottom of the stage (where the specimen sits). For high-resolution light microscopy, inverted setups are preferred as it gives more control of the objective and stage. 
A

Coherent

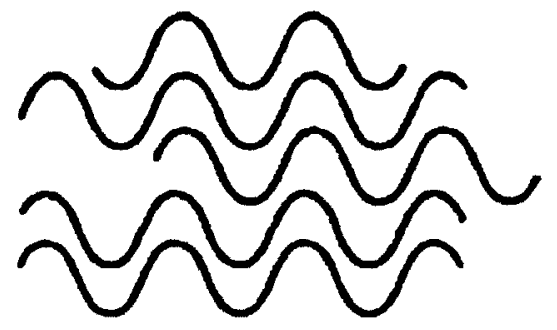

Noncoherent

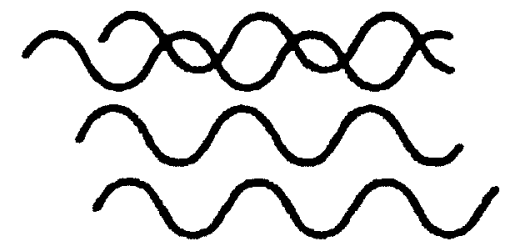

B

Ocular (eyepiece)

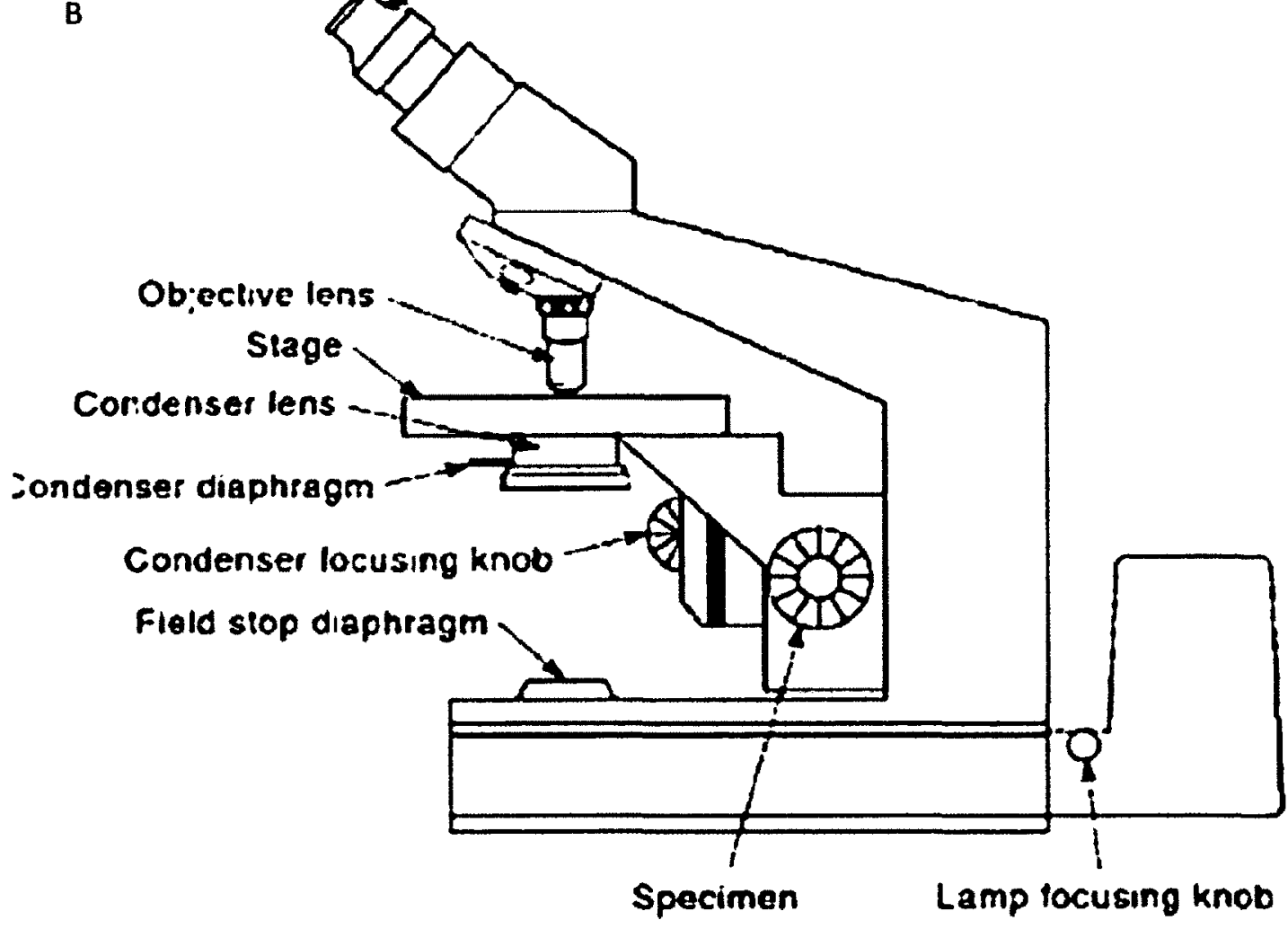

Figure 2.8: (a) Graphical representation of coherent (in phase) and incoherent (out of phase) light; and (b) typical setup of a light microscope. In contrast, an inverted microscope has the objective below while the light source and condenser lens are situated above the stage $\mathrm{e}^{110}$. 
Abbe also demonstrated that the resolution in light microscopes is limited by diffraction (considering light as a wave). Resolution is described as the point where two separate images merge and cannot be further distinguished from one another (which would further be described by the Rayleigh criterion ${ }^{111}$ ). (Figure 2.9). In terms of the Rayleigh criterion this means that the resolution limit is where the center of an airy disk generated by the diffraction of light as it interacts with a point source overlaps with the first order reflection of a second point source.

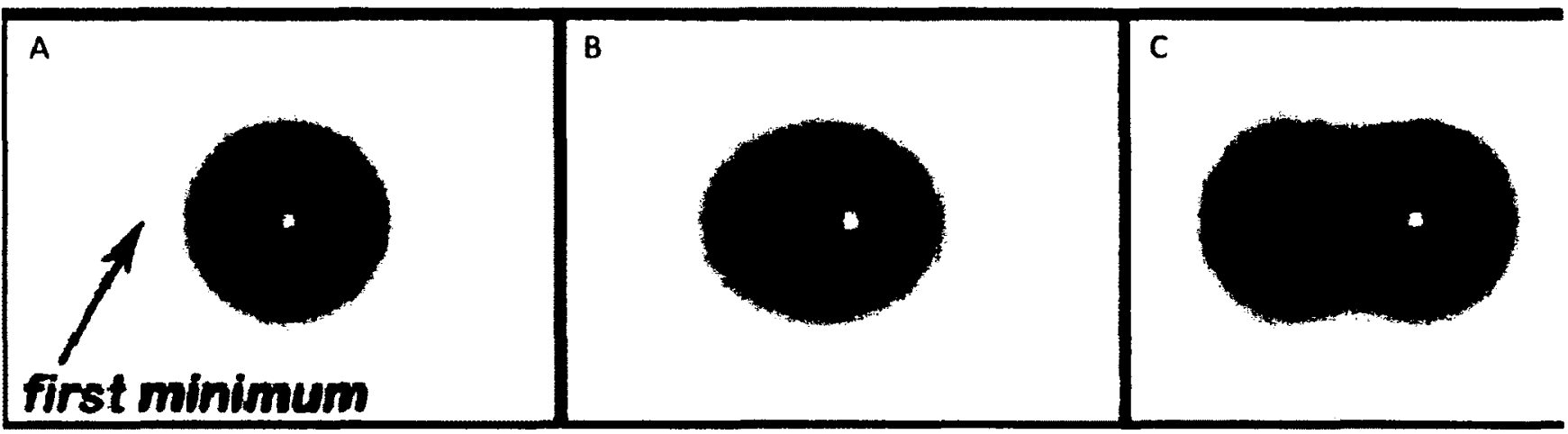

Figure 2.9: Graphical representation of resolution, where in (a) and (b) two separate points cannot be resolved, while in (c) the limit of resolution is approaching where the two points are becoming increasingly distinguishable ${ }^{112}$.

Lateral resolution (d) is the minimum distance where two points in an image can be distinguished before becoming merged the $x-y$ direction (see Figure 2.9). It is a function of wavelength of light $\left(\lambda_{0}\right)$, and numerical apertures of the objective and condenser $\left(\mathrm{NA}_{\text {obj }} \text { and } \mathrm{NA}_{\text {cond }} \text {, respectively }\right)^{113}$. 


$$
d=1.22 X \frac{\lambda_{\mathrm{o}}}{N A_{\mathrm{obj}}+N A_{\text {cond }}}
$$

The Numerical Aperture is described as the product of the sine half angle of the light cone either at the objective lens (from the specimen) or the condenser lens (from source) and the refractive indices of the solution used to mount the sample between the objective lens or condenser lens and stage ${ }^{113}$. In the case where the numerical apertures for the objective and condenser are the same (such as in the case of confocal microscopes), Equation 7 can be reduced to $8^{113}$. For conventional microscopes (including laser confocal), assuming a wavelength of $500 \mathrm{~nm}$ and a NA of 1.40 , the lateral resolution limit is ca. $2 \mu \mathrm{m}$ :

$$
d=\frac{0.61 \lambda}{N A}
$$

The axial resolution is similar to lateral resolution but is in the z-direction. During illumination of the sample, the intensity of light absorbed or emitted (fluorescence) extends above and below the sample in a cone shape in both directions. The axial

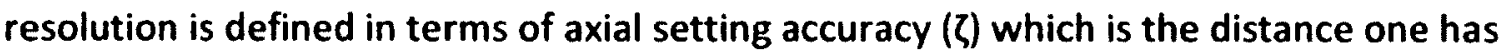
to move the fine focus before the image of an infinitely thin sample changes in perception $^{113}$. The axial setting accuracy is calculated using Equation 9:

$$
2 \zeta=\lambda /\left(4 n X \sin ^{2}\left(\frac{u}{2}\right)\right.
$$


Where $\lambda=$ wavelength of light, $n=$ refractive index of mounting solution, $u=$ half angle of the cone of light emitted from the sample (at the objective lens). The axial resolution can be typically optimized by the microscope software and the user has little control over it.

Lateral and axial resolution can be maximized by controlling the optics of the microscope, which include controlling the depth of field, geometric spreading along the lateral and axial planes, coherence of light, the emitted or absorbed light, controlling photobleaching, etc. Thus, it is not a trivial matter to perform quantitative measurements on a light microscope. It requires much practice, patience and understanding of the limitations of the instrument.

Confocal microscopy was described first in a patent by Marvin Minsky in $1957^{113}$. In comparison to the light microscope, in a confocal microscope, the condenser and objective lenses are replaced by a single lens $(O)$ and thus there is only one NA (resolution calculated according to Equation 8). Pinholes replace the condenser and are used to select specific wavelengths of light and send a very thin and focused coherent wave to the dichromatic mirrors (dichroic). In a confocal microscope (Figure 2.10), the entrance pinhole (A), focused light at the specimen (D), the specimen (S), and exit pinhole $(B)$ are all on the same plane, hence 'confocal'. The light is generated through a light source, such as a UV lamp or lasers, after which the entrance pinhole carefully selects the amount of coherent light to pass. The light is then split by a dichromatic mirror $\left(M_{1}\right)$, sent to the objective lens $(O)$ and focused onto the sample stage. The emitted (or reflected) light is returned the dichromatic mirror, where a specific 
wavelength is selected (for absorbance or fluorescence) which is then focused onto the exit pinhole, which filters out-of-focus light. The resultant light is sent to the photomultiplier or charge coupled device $(P)$, which generates an image dependant on the signal intensity ${ }^{113}$. 


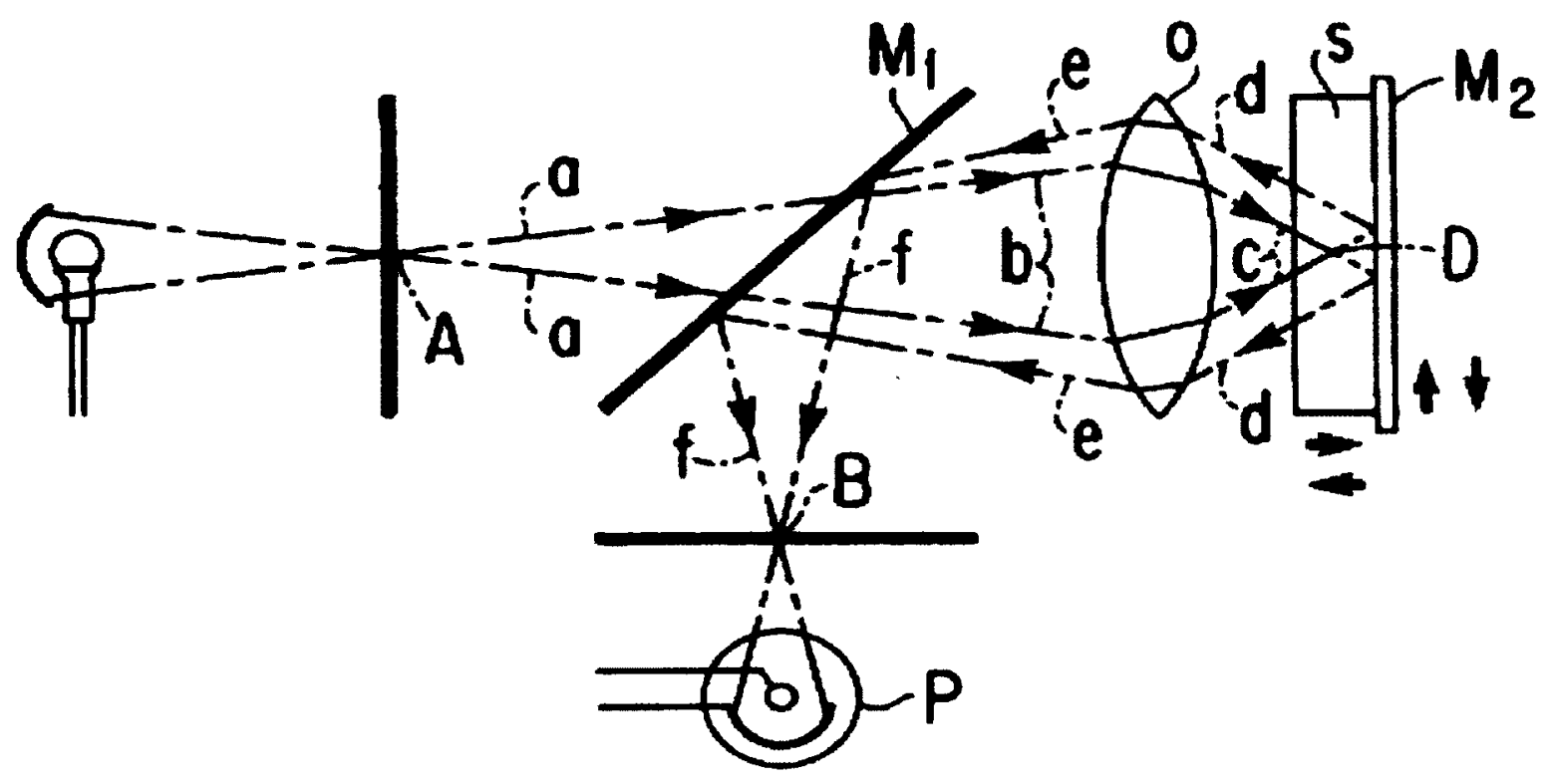

Figure 2.10: Typical setup for a confocal microscope, where light generated (by laser or lamps) passes through an entrance pinhole (A) to remove out of phase light. The light then passes through a dichromatic mirror $\left(M_{1}\right)$ which splits and allows only selected wavelengths (by user) to pass through. The light is then focused by an objective $(0)$ onto the focal plane (D) of the specimen (S). The reflected light is then sent through the objective to the dichromatic mirror which reflects selected wavelengths (by user) onto an exit pinhole $(B)$ which filters incoherent light. The resultant light is captured by a photocell or a charge coupled device $(P)$ to generate an image of relative light intensities over 2-dimensional space. Moving the focal plane in the axial direction by moving the stage (S), different axial regions of the sample can be imaged ('slices). These slices can be combined to generate a 3-dimentional image of the sample ${ }^{113}$.

By using small pinholes, one can improve the light collected by filtering out-of-focus

light, and thereby, improve both lateral and axial resolution. Due to the focused light

from the pinholes, dichromatic mirror selecting precise wavelengths to be transmitted

and reflected, and the fixed objective lens which focuses light onto the focal plane, 
there is very little illumination of regions outside focal plane, thus allowing for very high resolution imagery. By moving the stage in the axial plane and changing the focal plane, a 3-dimensional image can be generated of fluorescent or absorbent material. Using lasers as the source, which produce highly monochromatic (coherent) and high intensity light of a specific wavelength, has made confocal microscopy a fundamental tool for high-resolution quantitative studies on chemical and biological samples in 2 and 3 dimensions ${ }^{114}$.

\subsection{Experimental}

\subsubsection{Materials}

Microscope quartz slides $(75 \mathrm{~mm} \times 25 \mathrm{~mm})$ were purchased from VWR.

Poly(diallyldimethylammonium chloride) (PDDA, Mw 100,000-200,000Da), poly(sodium 4-styrene-sulfonate) (PSS, Mw 100,000Da), Calf Thymus DNA (CT, sodium salt, Type I) and sulforhodamine $B(S B)$ were purchased from Sigma and were used as received. Tetramethylrosamine (TMR) was purchased from Invitrogen and was used as received. The sulforhodamine B aptamer (SA sequence: 5'-CCG GCC TAG GGT GGG AGG GAG GGG GCC GG-3') and random oligonucleotide (RO sequence: 5'-GAC CTA TGA TAG CAT CAG TCG CAT CAG TC-3') were synthesized on a merMADE 6 (Bioautomation corporation) using standard phosphoramidite chemistry ${ }^{115}$. All glassware was rinsed $5 X$ with distilled and $5 X$ with double deionized water). 


\subsubsection{Methods}

Deposition of polyelectrolyte layers: Deionized water was used for all experiments.

Quartz slides were prepared by treatment with a solution of $\mathrm{H}_{2} \mathrm{O}_{2}: \mathrm{NH}_{3} \mathrm{OH}: \mathrm{H}_{2} \mathrm{O}(1: 1: 5)$ for 10 minutes at $70^{\circ} \mathrm{C}$. The same treatment and subsequent Ibl deposition methodology was used for the quartz/gold crystals for the quartz crystal microbalance experiment. After pretreatment, the slides were washed with water and were then dipped successively in solutions of oppositely charged polyelectrolytes, starting with PDDA $(5 \mathrm{mg} / \mathrm{mL}$ in $0.2 \mathrm{M} \mathrm{NaCl})$ and then PSS $(5 \mathrm{mg} / \mathrm{mL}$ in $0.2 \mathrm{M} \mathrm{NaCl})$ for 20 minutes each. The slides were washed with copious amounts of water in between each deposition step. This procedure was repeated 5 times yielding 5 bilayers of PDDA and PSS (See Figure 1). Slides with only these bilayers (hereafter named PDDA/PSS) served as controls in later experiments and later chapters in this Thesis. For the films containing the sulforhodamine B aptamer (PDDA/PSS/SA), a random oligonucleotide (PDDA/PSS/RO), or calf thymus DNA (PDDA/PSS/CT), the next 5 bilayers were deposited with DNA as the anionic layer, in place of PSS. In each case, the slide was immersed in the DNA solution $(6 \mu \mathrm{M} \text { for } \mathrm{SA} ; 11 \mu \mathrm{M} \text { for RO; } 0.04 \mu \mathrm{M} \text { for } \mathrm{CT})^{116}$ in $20 \mathrm{mM}$ Tris- $\mathrm{HCl}(0.2 \mathrm{M} \mathrm{NaCl}, \mathrm{pH} 7.4)$ for 20 minutes at room temperature. These films were terminated with an additional bilayer of PDDA/PSS to embed the DNA. The films compositions were (PDDA $)_{5}(\mathrm{PSS})_{5}$, $(\mathrm{PDDA})_{11}(\mathrm{SA})_{5}(\mathrm{PSS})_{6},(\mathrm{PDDA})_{11}(\mathrm{RO})_{5}(\mathrm{PSS})_{6},(\mathrm{PDDA})_{11}(\mathrm{CT})_{5}(\mathrm{PSS})_{6}$. In all cases the oligonucleotides (SA, RA, and CT) were embedded in between bilayers of PDDA/PSS. Films were left to dry open to air at room temperature for at least five hours before dye binding experiments were performed. 
Dye binding and dissociation constant $\left(K_{d}\right)$ experiments: Prior to dye binding, all films were heated at $70^{\circ} \mathrm{C}$ for 10 minutes to unfold any undesirable DNA conformations and allowed to cool to RT. Films were dipped in $2 \mathrm{mM}$ sulforhodamine $B$ (SB) or tetramethylrosamine (TMR) solution $(0.1 \mathrm{M} \mathrm{KCl)}$ for 30 minutes and then washed with copious amounts of water until washings were no longer colored. UV/vis spectra of the films were obtained on a Cary 300 UV/vis spectrophotometer. To minimize electrostatic binding of the dye directly to the outermost layer of the polymer film, the film tested with negatively charged SB dye was terminated with PSS while the film used for testing positively charged TMR was terminated with PDDA.

Reusability and stability of PDDA/PSS/SA was tested by immersing the slides in deionized water either at $40^{\circ} \mathrm{C}$ or $70^{\circ} \mathrm{C}$ for ten minutes or 24 hours at room temperature, and then examining the UV-Vis spectrum for loss of the dye or DNA bands ( $570 \mathrm{~nm}$ and $260 \mathrm{~nm}$, respectively). Films were then re-immersed in dye solution and binding was compared to the sample before heating, by UV-Vis.

Two types of dissociation constant experiments were performed. Initially, UV/vis spectroscopy was used as follows. PDDA/PSS/SA films were dipped in sulforhodamine B $(0.1 \mathrm{M} \mathrm{KCl})$ at concentrations of $0.001-10 \mathrm{mM}$ for 30 minutes and washed with copious amounts of water before obtaining the UV/vis spectrum. The results were compared to those obtained using a Quartz Crystal Microbalance (QCM) from Stanford Research Systems (QCM200, 5MHz AT-cut quartz crystal oscillator with $0.1 \mathrm{sec}$ gate time). The quartz crystal was functionalized using the same preparation conditions as described for the quartz slides. Film growth was monitored by the change in relative frequency while 
carefully nulling any capacitance by manual control of the bias voltage required by the varactor. For the dissociation constant experiment, $1 \mathrm{~mL}$ solutions of varying SB concentrations $(0.001-10 \mathrm{mM}, 0.1 \mathrm{M} \mathrm{KCl}$,$) were placed on the quartz crystal for thirty$ minutes, after which time the crystal was washed with copious amounts of water, dried under argon for 3 minutes and the frequency change was noted. For both experiments, the dissociation constants were evaluated by minimizing the residuals values between calculated and observed experimental $\Delta$ absorbance, or $\Delta$ frequency data using the solver feature of Microsoft Excel ${ }^{117}$.

Microscopy: All microscopy was performed in air. Confocal Microscopy, to characterize the distribution of SB within the polymer films, was performed on a Zeiss LSM510 (532 $\mathrm{nm}, 3 \%$ laser intensity for the films PDDA/PSS/SA and PDDA/PSS/RO, 5\% laser intensity for the PDDA/PSS/CT film) with a Plan-Acochromat 63x/1.4 Oil Dic objective with LP950 filter, exciting at $556 \mathrm{~nm}$ and collecting image data at $575 \mathrm{~nm}$. Atomic Force Microscopy (AFM), to characterize surface topography of the films, was performed on a Veeco Instruments $\mathrm{DI}-3100$ in both contact and tapping mode. AFM was also used to determine film thickness by creating a scratch on the polymer surface with a diamond etched knife on an Ntegra SFCO50LNTF AFM head.

Time of Flight-Secondary Ion Mass Spectrometry (ToF-SIMS): A ToF-SIMS IV instrument from lonTOF GmbH (Univ. of Alberta) was used to acquire compositional depth profiles of the films. Both positive and negative ion profiles were collected from several points on the surface. $\mathrm{ACs}^{+}$ion beam at $500 \mathrm{eV}$ primary energy was used for sputtering to collect negative secondary ions, and an $\mathrm{O}_{2}{ }^{+}$ion beam with same energy was employed 
for the positive polarity. The sputter beams were rastered over an area of about $400 \times 400 \mu \mathrm{m}^{2}$. The signal of the secondary ions was collected from the central area of about $200 \times 200 \mu \mathrm{m}^{2}$ with $64 \times 64$ pixels. The analytical gun used to generate the secondary ions from the central area used a chopped $15 \mathrm{keV} \mathrm{Ga} \mathrm{ion} \mathrm{beam} \mathrm{in} \mathrm{a} \mathrm{high-}$ current bunch mode. Charge compensation by electron flooding was also employed during profiling since the samples were non-conductive.

\subsection{Results and Discussion}

Layer-by-Layer (|bl) self-assembly of polyelectrolyte films is emerging as a method of choice for the preparation of thin films for medicine, biology, and material science. As aptamers can be generated for nearly any target, incorporation of these nucleic acids as recognition elements within these multilayer films is of great interest. One concern, however, is that the electrostatic interaction of the aptamer with the surrounding polyelectrolyte matrix could potentially interfere with target recognition, especially in an aptamer that needs to be highly structured to effectively bind. This study sought to confirm the feasibility of embedding aptamers within a polyelectrolyte matrix for eventual use in our smart fertilizer systems. The sulforhodamine B aptamer, which adopts a g-quadruplex structure ${ }^{118}$, was chosen as our model for this study. In order to assess the binding ability of this aptamer when immobilized in a polymeric matrix, thin films were prepared using the LbL approach (Figure 2.11). Four types of films were prepared $^{119}$ : the sulforhodamine B aptamer film, PDDA/PSS/SA, and three controls, 
PDDA/PSS (polymer layers only), PDDA/PSS/RO (containing a random oligonucleotide), and PDDA/PSS/CT (containing calf thymus DNA). In all films, 5 bilayers of PDDA and PSS were first deposited on a quartz slide. The growth of the film with each bilayer could be monitored via UV/vis (PSS 220 $\mathrm{nm}^{120}$ ). For PDDA/PSS/SA, PDDA/PSS/RO, and PDDA/PSS/CT, the next 5 bilayers were deposited using DNA as the polyanionic electrolyte, either the sulforhodamine B aptamer, a non-specific single-stranded random oligonucleotide or calf thymus DNA, respectively. Growth of the films during DNA deposition could be monitored by UV-Vis as well $(260 \mathrm{~nm})$. All films were terminated with an additional PDDA/PSS layer to embed the DNA within the film and also to minimize any electrostatic interaction between the charged dye and the film. Small molecules, such as the dye target, are known to diffuse through the layers of polyelectrolyte films, while larger biomolecules, nucleases for example, are not ${ }^{121}$. Thus, embedding the aptamer within the film should not impede dye access to the aptamer, but may serve to protect the aptamer from degradation. 
A

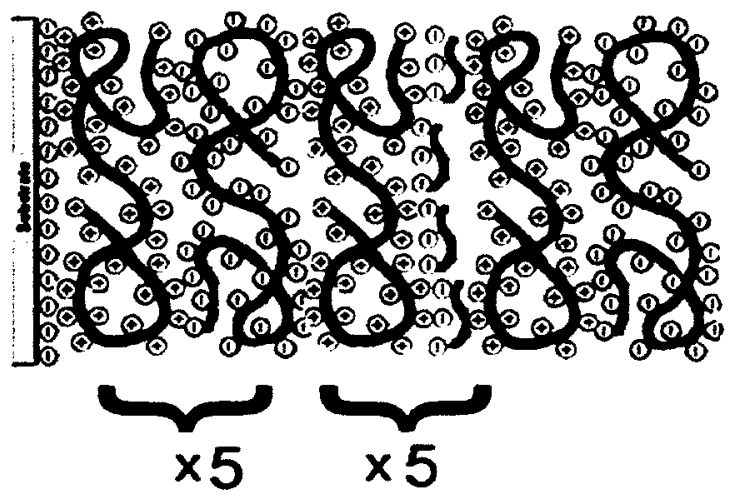

B Random Oligo (RO)

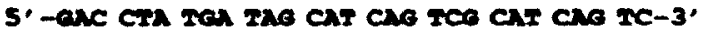

Poly(styrene sulfonate) (PSS)<smiles>CCCc1ccc([O+])cc1</smiles>

Poty(diallyldiammonium) (PDDA)<smiles></smiles>

$\mathrm{H}_{3} \mathrm{C}^{\prime} \mathrm{CH}_{3}$
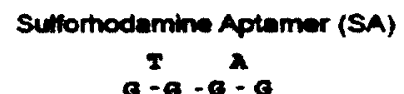

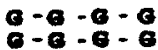

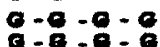

a

c:a

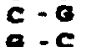

: $: c$

c: :

s. 3.

sufforhodamine B (SB)

Tetramettytrosamine (TMR)

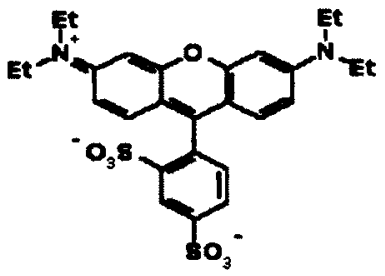

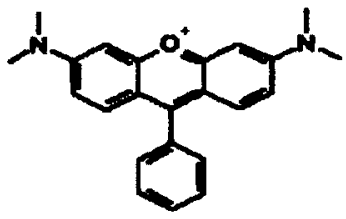

Figure 2.11: Experimental Overview. A) Thin film composition ${ }^{122}$. In PDDA/PSS/SA, 5 bilayers of PDDA (blue) and PSS (red) are followed by 5 bilayers of PDDA and SA (green). The films are capped with a final bilayer of PDDA/PSS. The films PDDA/PSS/CT and PDDA/PSS/RO are prepared with a similar composition except that Calf Thymus DNA $(C T)$ or random oligonucleotide DNA (RO) is substituted for SA in the second 5 bilayers. PDDA/PSS films without any DNA were also prepared and used as controls. The film illustration was modified from reference 1. B) Components of the Experiment. The sequence of the random oligonucleotide (RO), the sequence and structure (showing the predicted g-quadruplex) of the sulforhodamine $B$ aptamer (SA), and structures of the polyelectrolytes, PSS and PDDA, are presented. The structures of the target, Sulforhodamine B (SB) and Tetramethylrosamine (TMR; a related dye with no affinity for the Sulforhodamine aptamer) are also shown. 


\subsubsection{Atomic Force Microscopy of Functional Bilayer Films}

AFM was used to determine that the DNA-containing films were approximately $80 \mathrm{~nm}$ in thickness. This value is higher than what would be expected for a pure PDDA/PSS film at this salt concentration, demonstrating that the DNA layers have a sizeable effect on film properties ${ }^{123}$. Figure 2.12 shows AFM of the PDDA/PSS/SA and PDDA/PSS/CT films before annealing where the films have been terminated with the oligo layer (in comparison to terminating with PDDA/PSS bilayer). As can be seen, SA and CT have dramatically different morphologies. This would suggest that when aptamers are embedded in our polyelectrolyte films, there will be a change in film morphology which is likely dependant on the differences in size and secondary structures of the DNA. Due to this, we decided to use a strand which is the same length as SA, but which is missing the G-rich region required for target binding $(R O)$ as a more appropriate control for our experiments. These images correlate with the data obtained by the CLSM (see Section

\subsection{3).}




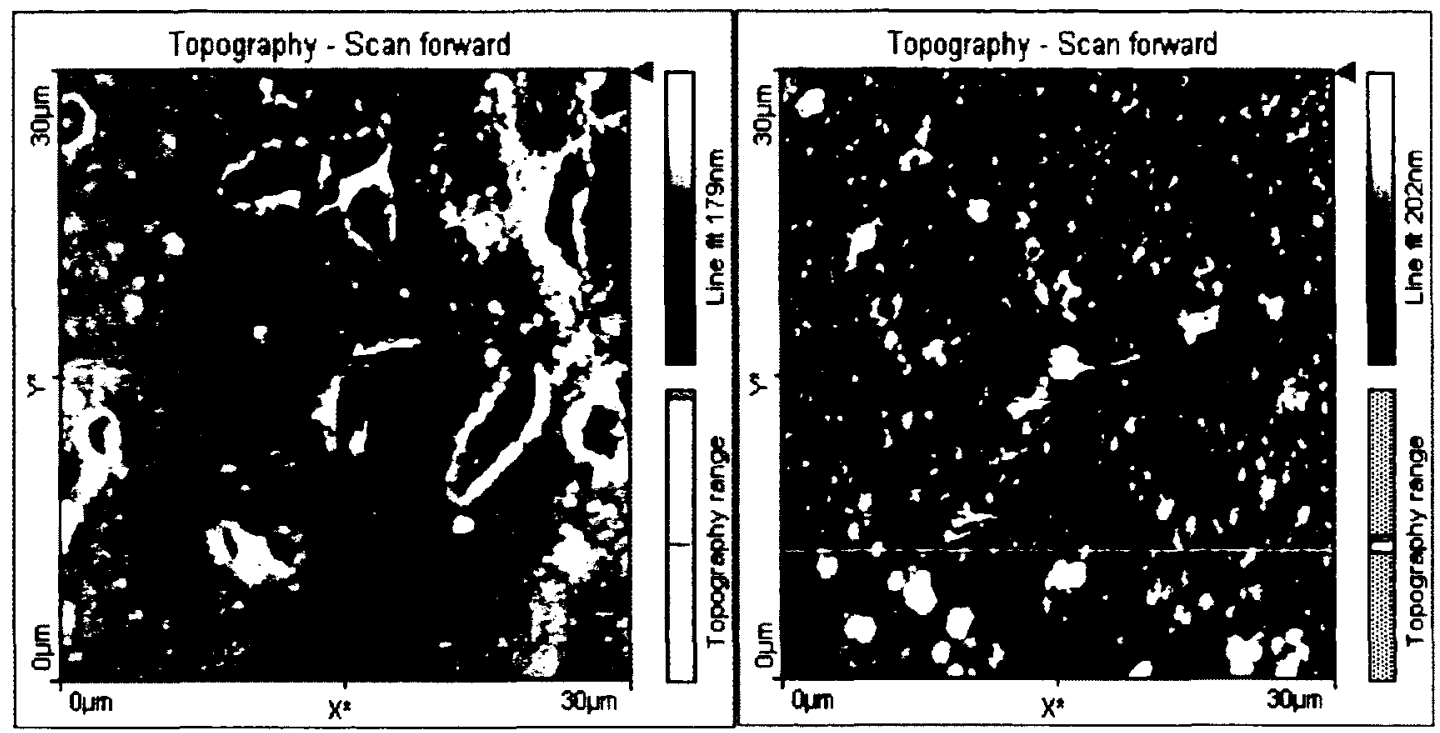

Figure 2.12: AFM image of films terminated with CT (left) and SA (right) showing the different morphologies of the two films.

The surface roughness also increased as a result of embedding DNA into the films

(Figure 2.13).

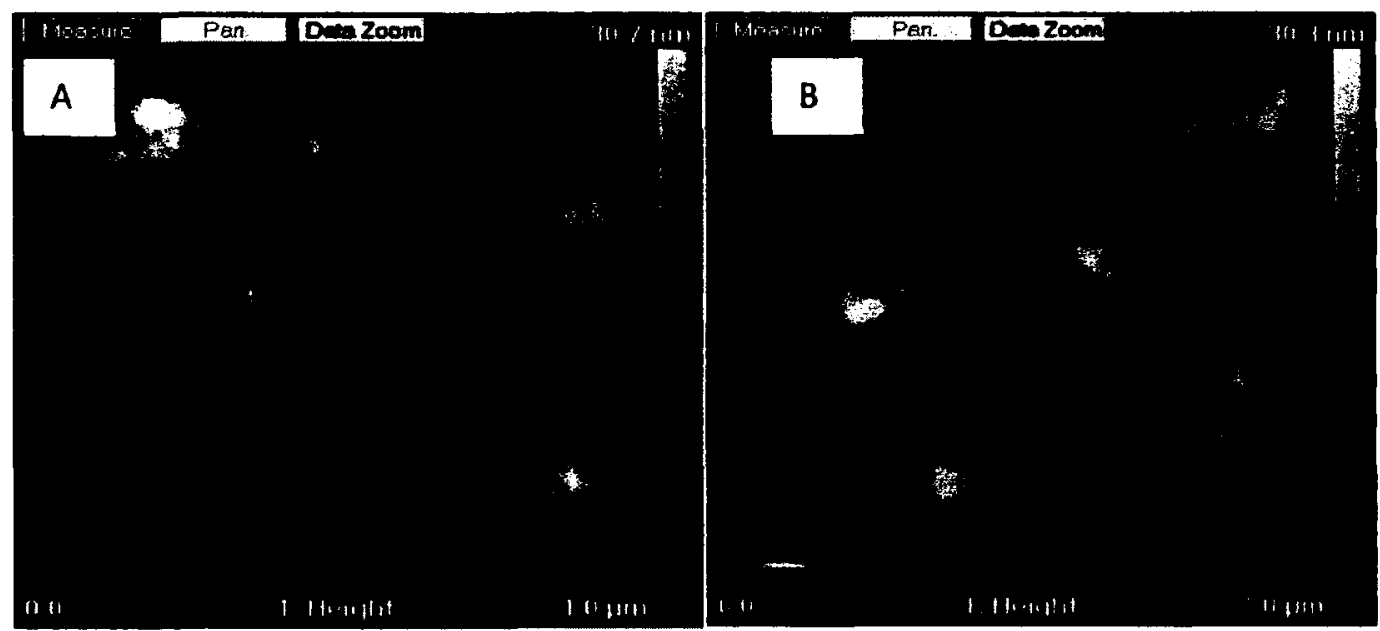

Figure 2.13: AFM of (a) PDDA/PSS; and (b) PDDA/PSS/SA films. An increase in surface roughness (bright regions) can be seen in the PDDA/PSS/SA films, correlating with the AFM data shown in Figure 2.9(b). 


\subsubsection{UV/Vis Spectrophotometry of Aptamer Functional Bilayer Films [qualitative}

analysis]

Given the strong absorbance of the SB dye in the visible region, UV-Vis spectroscopy was employed as an initial measure of whether the target recognition of the aptamer was maintained while it was embedded within these polyelectrolyte films. After incubation with the dye, the quartz slide was placed beside the sample window of the UV-Vis spectrometer, ensuring that it was perpendicular to the holder base to ensure consistency in the path length. Figure 2.14 is a comparison of the spectra for the various films after dye binding. Clearly, the spectrum of PDDA/PSS/SA shows a larger signal from the SB dye absorbance $(\sim 570 \mathrm{~nm})$ than the controls. However, as the dye, DNA, and polymer peak intensities varied from film to film, ratios of dye peak height to polymer or DNA peak height were used to better quantify the affinity of the films for the target, as these ratios showed less variation from sample to sample (Table 2-1). 


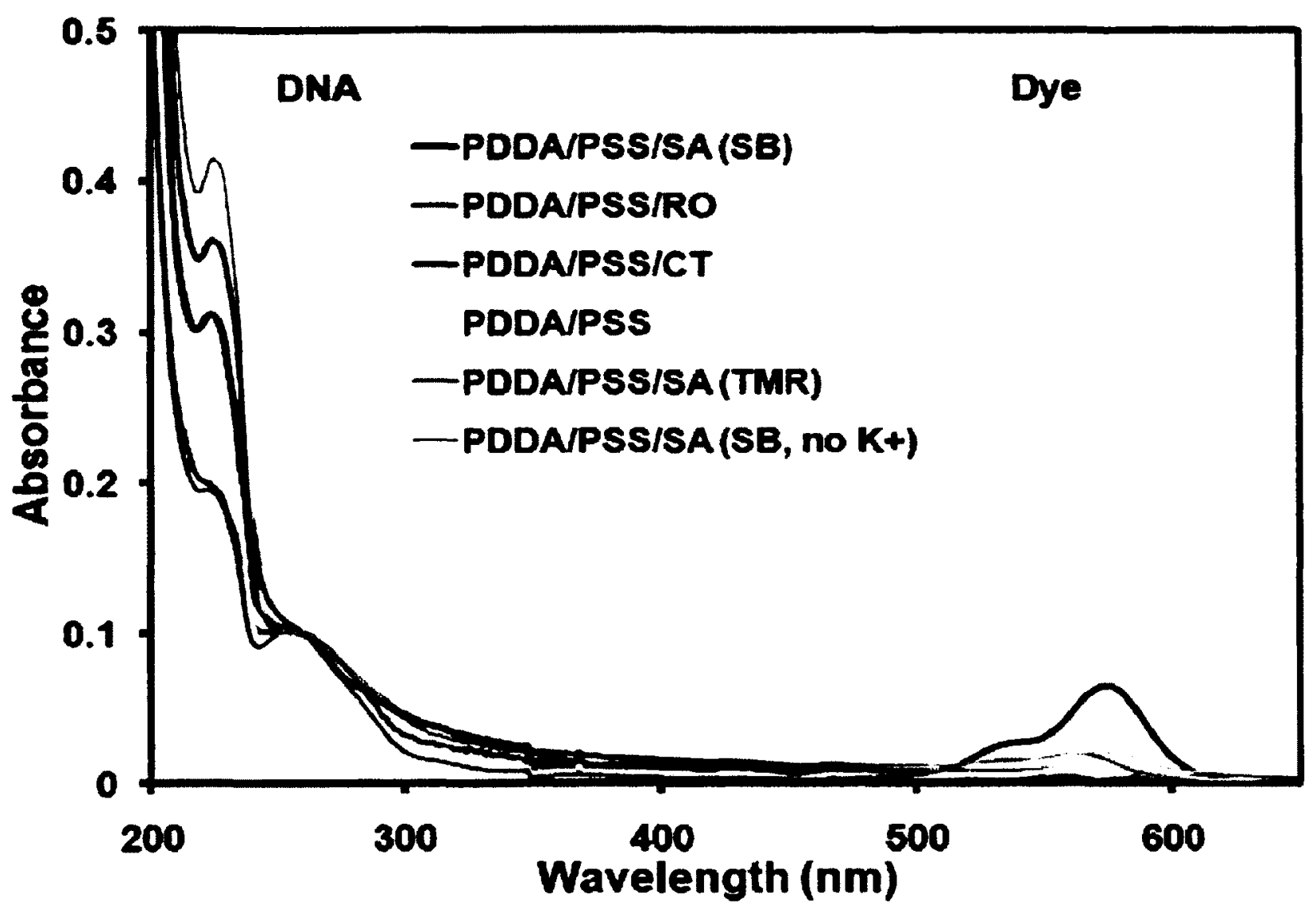

Figure 2.14: Overlay of UV-Vis spectra of the polyelectrolyte films. DNA containing films are normalized at $260 \mathrm{~nm}$. Black: PDDA/PSS/SA exposed to Sulforhodamine B (SB) Red: PDDA/ PSS/RO exposed to SB, Green: PDDA/PSS/CT exposed to SB, Yellow: PDDA/PSS alone exposed to SB, Blue: PDDA/PSS/SA exposed to Tetramethylrosamine (TMR), Orange: PDDA/PSS/SA exposed to SB, but without $K^{+}$. The regions of the spectra assigned to DNA and dye absorbances are highlighted. Upon immersion of the films in the dye solution, the aptamer films show the greatest degree of dye binding as evidenced by the largest peak at $570 \mathrm{~nm}$. 
Table 2-1: Comparisons of Dye, Polymer, and DNA Absorbance Values for the Films of this Study.

Sample Composition

\begin{tabular}{|c|c|}
\hline $\begin{array}{l}\text { Ratio' of SB Dye Peak Height } \\
\text { to Polymer Peak Height } \\
(570 \mathrm{~nm}: 220 \mathrm{~nm})\end{array}$ & $\begin{array}{l}\text { Ratio" of SB Dye Peak Height } \\
\text { to DNA Peak Height } \\
(570 \mathrm{nm:260} \mathrm{nm)}\end{array}$ \\
\hline
\end{tabular}

PDDAPSS

PDDAPSS/CT

PDDA /PSS/RO

PDDA/PSS/SA (with $K^{+}$)

PDDA/PSS/SA (without $K^{+}$)
$0.12(0.01)$

$0.08(0.01)$

$0.12(0.03)$

$0.21(0.06)$

$0.09(0.01)$
$0.23(0.02)$

$0.34(0.09)$

$0.70(0.01)$

$0.26(0.03)$

${ }^{a}$ Average of at least three measurements, standard deviation in parentheses

When PDDA/PSS films alone were tested for their ability to bind the dye nonspecifically, a small absorbance in the dye region was noted. This non-specific binding could be attributed to the fact that the dye, being negatively charged, will likely have some affinity for the positively charged PDDA. In these films, the ratio of the dye peak intensity to polymer peak intensity was approximately 0.12 . This serves as a baseline for non-specific dye binding in these films. When either the random oligonucleotide or calf thymus DNA is included in these films, the amount of non-specific binding (as measured by the dye to polymer ratio) is either less than, or equal to the baseline value found in the polymer films alone. The aptamer-containing film, PDDA/PSS/SA however, showed considerably stronger affinity for the dye, with a dye to polymer ratio of approximately 0.21. Similarly, if the dye absorbance was compared to the DNA absorbance at 260 $\mathrm{nm}$, the ratio of the peaks in the aptamer films was more than double that of the other 
films. Studies on the SA aptamer have indicated that the sequence forms a stacked $g$ quadruplex structure in the presence of $\mathrm{K}^{+}$, but not $\mathrm{Na}^{+}$and that this structure is essential for maximum dye binding ${ }^{124}$. Indeed, heating the PDDA/PSS/SA films to unfold any undesirable DNA structures before immersion of the film in the dye solution with a high concentration of $\mathrm{K}^{+}$was required for efficient dye binding; films immersed in $\mathrm{K}^{+}$free dye solutions showed levels of dye binding that were comparable to controls. (see Figure 2.11 and Table 2-1) These data suggest that the aptamer in PDDA/PSS/SA retains its ability to form the g-quadruplex structure in order to recognize and bind the sulforhodamine B target, over and above the small non-specific electrostatic interaction that the other films exhibit. In terms of concentration, using an extinction coefficient of 93,000 for the target dye, there is ca. 2.5 times more dye on the aptamer (with $\mathrm{K}^{+}$) films relative to the control films.

The effect of immobilization in the polyelectrolyte film on aptamer specificity was also investigated. In the original work on the SB aptamer, TMR dye was shown to be an inefficient competitor for binding, despite the structural similarities between the two

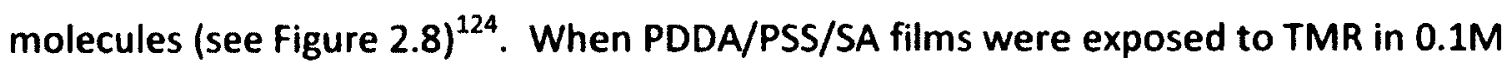
$\mathrm{KCl}$, only a low level of binding (dye to polymer ratio of 0.05 , dye to DNA ratio of 0.17 ), similar to the amount in the $\mathrm{K}^{+}$-free case, was detected (Figure 2.11). The specificity of the sulforhodamine aptamer was therefore unaffected by the polyelectrolyte matrix. 


\subsubsection{Confocal Microscopy of Functional Bilayer Aptamer Films [semi-quantitative}

analysis]

Given the intense fluorescence of the SB target, confocal microscopy could also be employed to assess the binding ability of the polyelectrolyte films. Confocal microscopy images of the thin films are shown in Figure 2.15. Images of PDDA/PSS/SA showed considerably more bright areas consistent with bound dye than did PDDA/PSS/RO or PDDA/PSS/CT. Given that the size of the bright spots is on the $\mu \mathrm{m}$ length scale, these are most likely the result of groups of aptamers binding to SB. The PDDA/PSS/SA and PDDA/PSS/RO films were taken under identical settings (laser intensity had to be increased by $2 \%$ in order to image the PDDA/PSS/CT films). Using standard photo processing software, the mean brightness of the PDDA/PSS/SA image was found to be twice that of the PDDA/PSS/RO image. This correlates with the UV/Vis data (section

2.3.2). The CT DNA films were very inhomogeneous but nevertheless show the lowest mean brightness and the least bright areas, and those that are present appear to be localized in defects on the films ${ }^{125}$. 

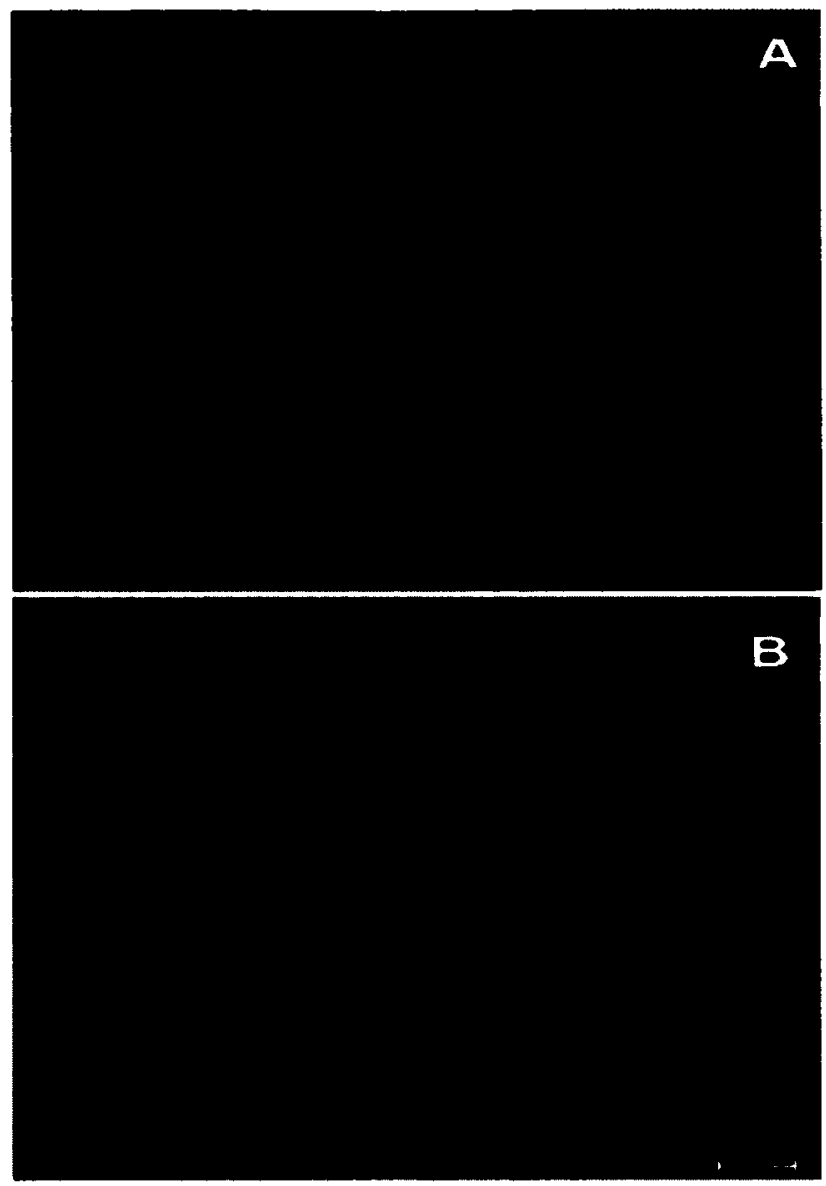

C

Figure 2.15: Confocal microscope images of the polyelectrolyte films after dye binding. Excitation $556 \mathrm{~nm}$, emission $575 \mathrm{~nm}$. White bars correspond to $10 \mu \mathrm{m}$ A) PDDA/PSS/RO B) PDDA/PSS/CT C) PDDA/PSS/SA. The random oligonucleotide-containing film (PDDA/PSS/RO -box A) shows few bright spots associated with the dye fluorescence. PDDA/PSS/CT films show very little dye fluorescence, with some localized nonspecifically within defects on the film surface. The film containing the aptamer (PDDA/PSS/SA -box C) shows the brightest fluorescent areas associated with bound dye. 


\subsubsection{Dissociation constant experiments $\left(K_{d}\right)$}

Dissociation constant $\left(K_{d}\right)$ is generally used as a measure of an aptamer's binding affinity or sensitivity. In solution, the sulforhodamine $B$ aptamer has a $K_{d}$ of ca. $0.7 \mu M^{126}$.

Dissociation constant experiments on our polyelectrolyte films, using absorbance changes at $570 \mathrm{~nm}$ with increasing dye concentration, proved difficult; in the PDDA/PSS/SA films, apparent dissociation constant values ranged from as low as $0.8 \mu \mathrm{M}$ to as high as $3 \mathrm{mM}$ (Figure 2.16). While the lower value is consistent with the $K_{d}$ for the sulforhodamine aptamer in solution, the higher value may be more representative of the non-specific dye interaction with the polymer films.

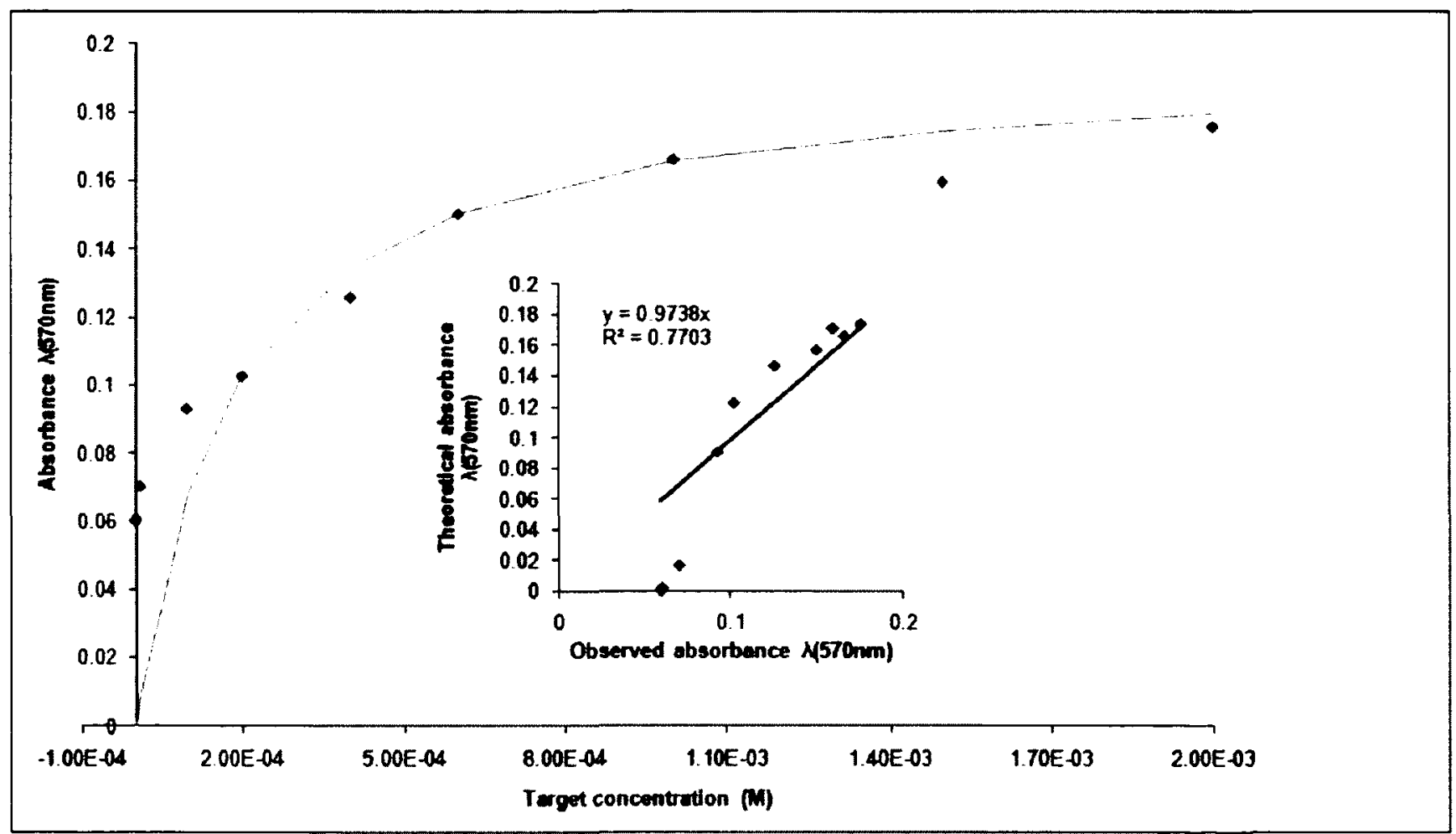

Figure 2.16 : Binding affinity curve using UV/vis of PDDA/PSS/SA films with varying concentrations of target (SB). The binding affinity using $U V / v i s$ ranged from $0.8 \mu M$ to $3 \mathrm{mM}$. 
Quartz Crystal Microbalance (QCM) experiments were used in an attempt to clarify this issue. QCM is a powerful, quantitative technique that detects molecular recognition events through frequency changes associated with minute mass increases on the crystal surface. QCM has been used to determine $K_{d}$ values for antibody-antigen and aptamertarget interactions ${ }^{127}$. PDDA/PSS/SA and PDDA/PSS/CT (negative control) films were deposited onto the crystal and frequency changes detected with increasing concentrations of SB dye were evaluated (Figure 2.17).

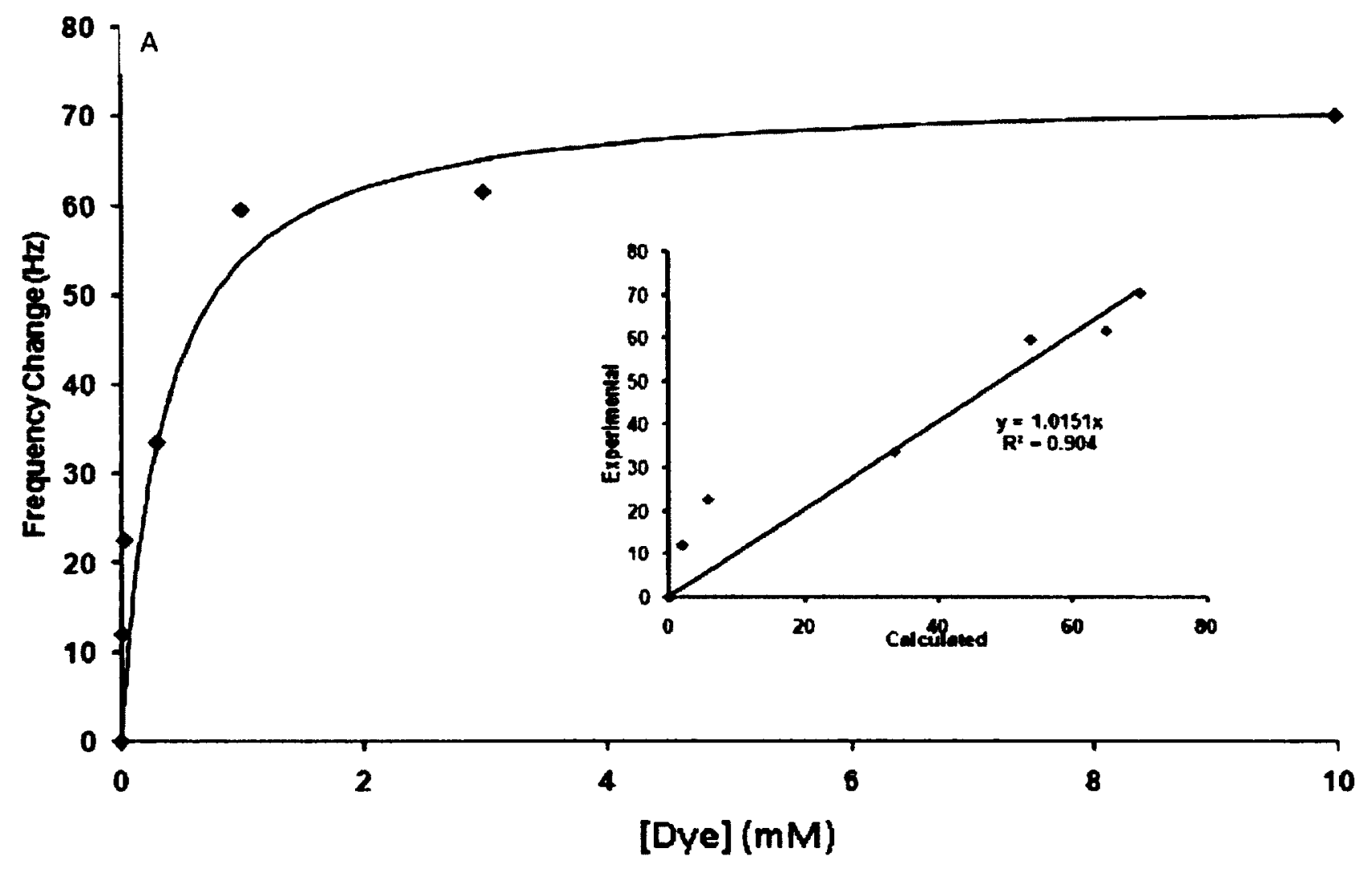




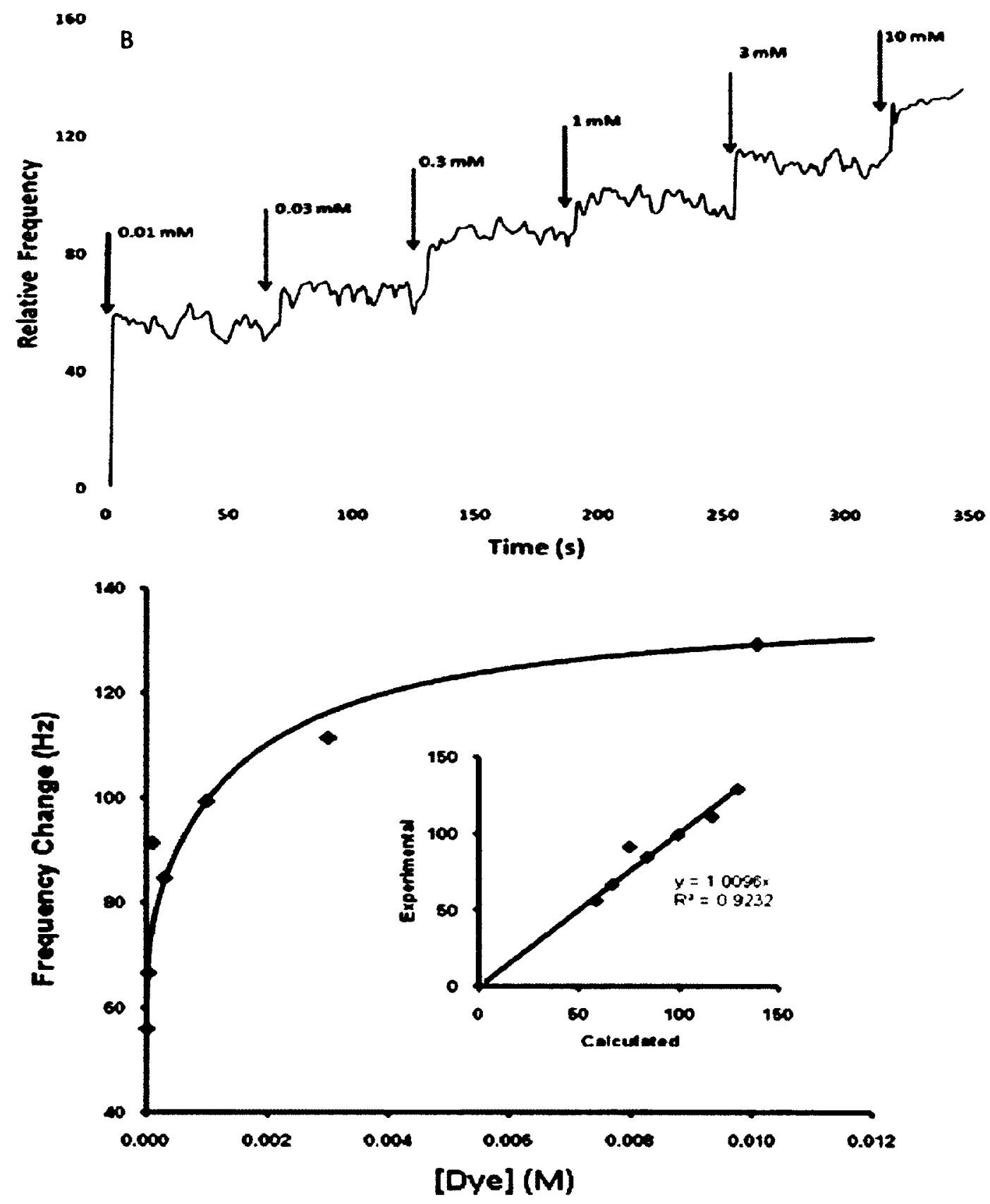

Figure 2.17: (a) Control QCM dissociation constant experiment on a PDDA/PSS/CT film. The line represents the fit to a single $K_{d}$ model. Inset: Values calculated using the single $K_{d}$ model vs. the experimental data.; (b) Representative data from QCM dissociation constant experiments. A PDDA/PSS/SA film on a quartz crystal was exposed to solutions of sulforhodamine $B$ dye and the relative frequency was plotted vs. the dye concentration. Top: Raw frequency data from the QCM experiment. Bottom: Plot of frequency over dye concentration. The line represents a two $K_{d}$ model (see text). Inset: Values calculated using the two $K_{d}$ model vs. the experimental data. 
The data for the SA films fit best to a model with two binding affinities, a specific $K_{d}$ of $16 \mu \mathrm{M}$ and a non-specific $K_{d}$ of $1.5 \mathrm{mM}$, consistent with the values obtained using UVVis. This suggests that the specific affinity of the aptamer has been only somewhat perturbed $\left(\sim 20 \times\right.$ higher $\left.K_{d}\right)$ by its incorporation into the polymer film. Data from control QCM $K_{d}$ experiments on the PDDA/PSS/CT films fit to a single binding affinity in the low $\mathrm{mM}$ range. These results confirm that there is a non-specific interaction between the films and the dye target. The high $K_{d}$ of this interaction, however, makes this nonspecific binding unlikely to affect the detection of targets at low concentrations.

\subsubsection{Time of Flight Secondary Ion Mass Spectrometry (Tof-SIMS) [quantitative analysis]}

To further confirm the specificity of the dye for the aptamer within the polyelectrolyte layers, the investigation of dye localization as a depth profile, rather than as a bulk measurement, was attempted. ToF-SIMS was used to confirm specificity and colocalization of the dye and the aptamer within the films. ToF-SIMS measurements were taken as a depth profile over several points on the film to examine whether the dye was co-localized with the aptamer layers, which would be consistent with specific aptamertarget binding (Figure 2.15). The $\mathrm{SO}_{2}{ }^{-}$secondary ion was monitored as it was present in both the PSS polymer and the SB dye, and $\mathrm{PO}_{3}{ }^{-}$was chosen to track the presence of DNA. $\mathrm{SiO}_{2}$ - was also monitored to demarcate the film/glass interface. In the absence of dye (Figure 2.18), all the $\mathrm{SO}_{2}^{-}$secondary ions are originating from the PSS within the 
film. The counts of $\mathrm{SO}_{2}{ }^{-}$secondary ion drop where the counts of $\mathrm{PO}_{3}{ }^{-}$ion rise. This is expected as there is no PSS in the layers of the film containing the aptamer. If SB dye had no affinity for the aptamer layers and was evenly distributed throughout the film, then after dye-binding the $\mathrm{SO}_{2}{ }^{-}$depth profile would be expected to show a similar trend. The regions of the film that contained both PSS and dye would show a higher $\mathrm{SO}_{2}^{-}$ion count, and the regions of the film where PSS is replaced by SA would show a lower $\mathrm{SO}_{2}^{-}$ count originating from the dye alone. After dye-binding, however, the $\mathrm{SO}_{2}{ }^{-}$secondary ion trace does not follow that trend and instead now tracks with the $\mathrm{PO}_{3}{ }^{-}$ion. This suggests that dye is predominantly co-localized within the aptamer layers of the film. As both the DNA and the dye are negatively charged, this co-localization is unlikely to be due to a simple electrostatic interaction, and these finding further corroborate that the aptamer maintained its ability to recognize the dye while embedded in the film. 

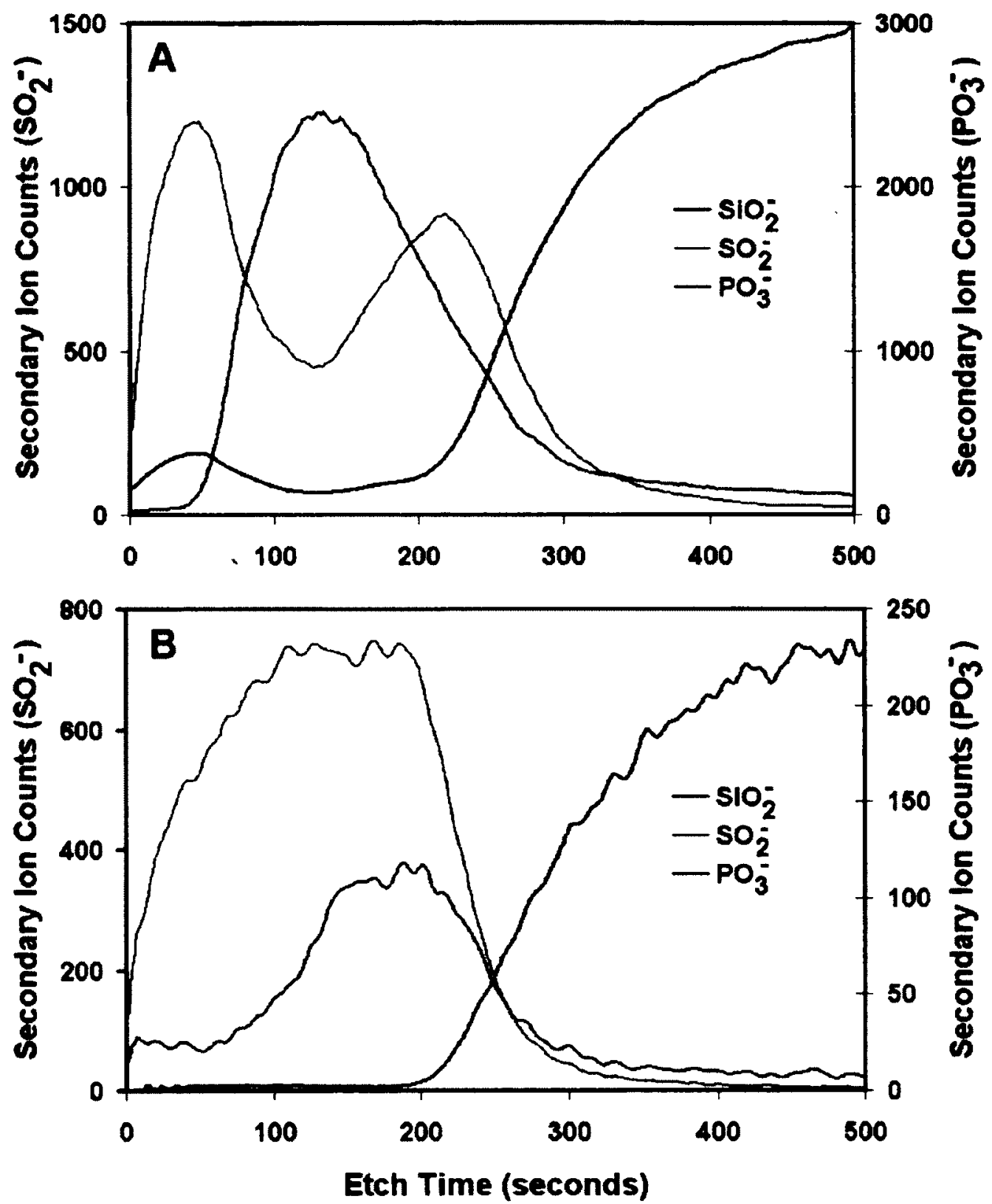

Figure 2.18: A TOF-SIMS sputter depth profile of PDDA/PSS/SA before (A) and after (B) dye binding. Red: $\mathrm{SO}_{2}{ }^{-}$, Green: $\mathrm{PO}_{3}$; $\mathrm{Blue} \mathrm{SiO}_{2}^{-}$. The $\mathrm{SO}_{2}^{-}$secondary ion is used to trace both PSS and SB, while the $\mathrm{PO}_{3}{ }^{-}$secondary ion is used to track the presence of the aptamer. The $\mathrm{SiO}_{2}{ }^{-}$ion counts have been reduced by a factor of 10 to fit on the same scale as the $\mathrm{SO}_{2}^{-}$. A) PDDA/PSS/SA alone: $\mathrm{SO}_{2}{ }^{-}$drops off dramatically as $\mathrm{PO}_{3}{ }^{-}$rises, and then rises again in the area directly above the slide surface. This trend was expected as the aptamer replaces PSS in the middle 5 bilayers of this film. B) PDDA/PSS/SA and dye: $\mathrm{SO}_{2}^{-}$counts reach a maximum at the same depth as do the $\mathrm{DNA}^{\prime} \mathrm{S} \mathrm{PO}_{3}{ }^{-}$counts, suggesting that the dye and the DNA are co-localized. 


\subsubsection{Stability and reusability of aptamer films}

Although DNA diffusion through polyelectrolyte films is expected to be minimal, a molecular beacon approach has recently shown that these multilayer films are somewhat permeable to short sequences of DNA ${ }^{128}$. Thus, PDDA/PSS/SA films were tested for their stability to leaching as well as their ability to be regenerated and reused. Little evidence of aptamer leaching was noted by examination of the peak at $260 \mathrm{~nm}$ when the films were left in deionized water over $24 \mathrm{hrs}$ at room temperature. PDDA/PSS/SA films containing the dye were also subjected to immersion in deionized water at $40^{\circ} \mathrm{C}$ and $70^{\circ} \mathrm{C}$; UV-Vis spectra determined that the dye, but not the aptamer, could be removed from the films under these conditions $(<1 \%$ loss of the signal at 260 $\mathrm{nm})$. Dye binding after regeneration was found to be comparable to binding in the original films (>95\% of the dye signal). Drying and air exposure also seemed to have little effect on the films. Aptamer-containing films left dry and open to air at room temperature for over three months still showed binding affinity when re-immersed in dye solution.

\subsection{Conclusions}

Using the sulforhodamine B aptamer as a model system, this study confirmed that the aptamer embedded in a polyelectrolyte matrix was still successful at binding its cognate target, with preserved specificity and only slightly perturbed affinity. This suggests that 
the sulforhodamine aptamer was able to retain its required g-quadruplex conformation while entrapped in the film. The multilayer polyelectrolyte films prepared by LbL assembly were found to be stable to aptamer leaching and reusable. The robust nature of these films, coupled with the ease with which they are prepared, indicates that LbL assembly is a practical and effective approach for the development of functional aptamer films. With results that suggest that aptamers are still functional within a polymer coating, we felt we could proceed with a polyelectrolyte/aptamer coating as the basis of our smart fertilizer system. In the next chapter, we will address our next question: Can aptamer-target binding affect the permeability of this polyelectrolyte coating?

\subsection{Acknowledgements}

Y.S thanks Ann-Fook Yang and Denise Chabot at the Agriculture and Agri-Food Canada Microscope facility for all their help in setting up the experiments and troubleshooting the CLSM. YS also thanks Dmitre Karpuzov at Alberta Centre for Surface Engineering and Science at University of Alberta for help with the ToF-SIMS. 


\section{Chapter 3. Microcapsules}

Sultan, Y.; DeRosa, M. Small, 2011, in press.

\subsection{Introduction}

In support of our smart fertilizer system, we have shown that aptamers retain their binding affinities (sensitivity and specificity) when embedded in polyelectrolyte films using the $\mathrm{lbl}$ approach (Chapter 2). We wanted to next understand the changes in permeability of polyelectrolyte films upon aptamer-target binding. To do this, we decided to use microcapsules built in the same way as our Ibl bilayer films (Chapter 2). Using this type of morphology will also be consistent with coating nitrogen fertilizers as they are, on average, spherical in shape. By utilizing the well-established fluorescence recovery after photobleaching technique on a laser scanning confocal microscope, we will be able to determine whether permeability increases or decreases as a result of our model aptamer binding to its target dye.

\subsubsection{Polyelectrolyte Microcapsules}

Polyelectrolyte microcapsules have attracted considerable interest for use as delivery vehicles for molecular payloads. Two main release strategies have been investigated in this regard; one depends on the stimuli-triggered decomposition or erosion of the microcapsule walls, while the other seeks to exploit the tunable permeability of the capsule coating. Polyelectrolyte capsules that undergo triggered decomposition have been prepared that are responsive to a range of stimuli, including glucose, biotin, 
exposure to high $\mathrm{pH}$, reducing conditions, ultrasound, magnetic fields, as well as UV, visible, NIR and IR irradiation ${ }^{129}$. Alternatively, permeability in Ibl films and coatings can be controlled by annealing temperature, ionic strength, type of counterion, $\mathrm{pH}$, solvent, polymer composition, and shell thickness ${ }^{130}$. Laser light illumination of embedded gold nanoparticles has also been shown to transiently affect the polyelectrolyte network leading to a reversible change in the capsule permeability ${ }^{131}$. Improved methods to control the permeability of polyelectrolyte capsules to allow for the triggered release of a molecular payload could have implications in a number of important areas, such as medicine (drug delivery) and agriculture (smart fertilizers) ${ }^{132}$. Furthermore, smart microreactors with selectively permeable walls that allow for controlled uptake of reactants could lead to more efficient preparation of a range of synthetic and biological materials. This added functionality could potentially be achieved by the inclusion of molecular recognition probes, such as aptamers, into the microcapsule layers. This chapter will look at the diffusion kinetics of a small molecule through aptamer-containing polyelectrolyte microcapsules. The FRAP technique (Section 3.1.2) will be used to monitor the changes in fluorescence recovery of a bleached spot inside the microscapsule before and after aptamer-target binding. By modeling the FRAP data, we will be able to calculate diffusion coefficients for the various microcapsules which will be a result solely of the changes in the lbl wall structure as a result of this aptamer-target pair. Different aptamer-target pairs will lead to different diffusion coefficients. 


\subsubsection{Fluorescence Recovery After Photobleaching (FRAP)}

FRAP or FRP is a quantitative fluorescence technique which allows the measurement of diffusion, dynamics of molecular mobility and chemical change of labeled species ${ }^{133}$. Due to the high resolution requirements for quantitative measurements, confocal laser scanning microscopy (CLSM or SLCM) is most commonly used to do FRAP experiments. In FRAP, a low intensity laser beam is used to scan a region of interest. A spot in the region of interest is photobleached by illumination from a high intensity laser beam. The fluorescence intensity is then measured at the same spot over a period of time and the recovery rate is used to calculate the dynamics of region of interest (see Figure 3.1). The data can be fit using first-order rate kinetics and a $t_{1 / 2}(s)$ and rate constant $\left(s^{-1}\right)$ can be calculated which allow for the determination of Diffusion $\left(\mu \mathrm{m}^{2} / \mathrm{s}\right)$ using Equation 3 which models 2D diffusion ${ }^{134}$ :

$D=\frac{\omega^{2} \ln (2)}{4 t_{1 / 2}}$

where $\omega$ is the bleach spot radius and $t_{y_{2}}$ is the fluorescence recovery half life in seconds (calculated from first order fit). 
A
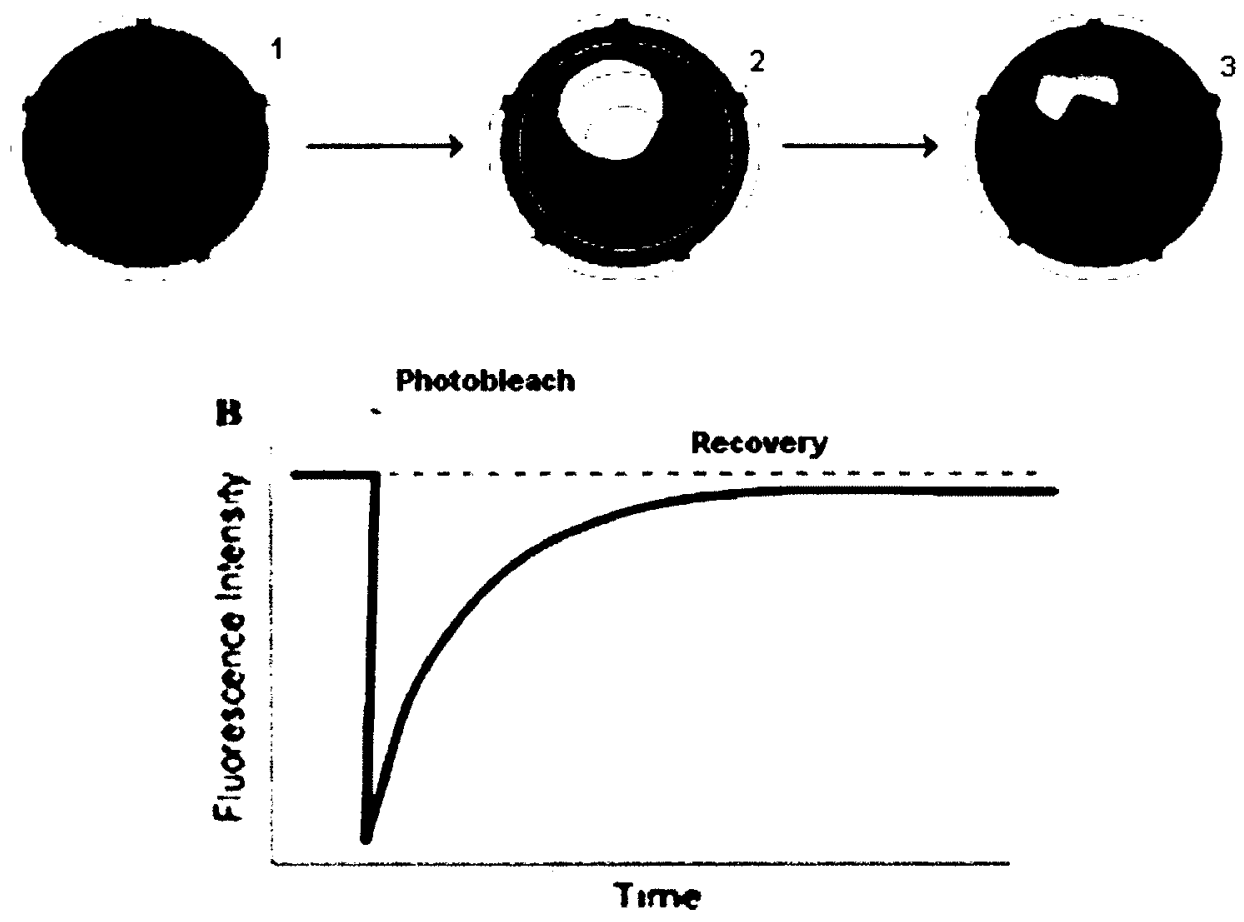

Figure 3.1: Typical FRAP experiment where a small fluorescent spot (ca. 1-2 $\mu \mathrm{m}$ ) is photobleached within a capsule $(>2 \mu \mathrm{m})(1 \rightarrow 2)$ and the resultant recovery of the fluorescence can be monitored over time (3). FRAP experiments can provide information on diffusion and kinetics of different mobile phases in the sample.

Over the last decade, FRAP has been used to study various polyelectrolyte systems, including microcapsules. Groups have looked at the release (diffusion) of various polyelectrolyte systems including drugs through various types of microcapsules ${ }^{135}$, polyelectrolyte DNA microcapsule release and degradation ${ }^{136}$, and stability (kinetics) of microcapsules ${ }^{137}$. FRAP is thus a powerful technique to probe the changes of permeability of our aptamer microcapsules since one can take high-resolution quantitative measurements of fluorescent samples and model diffusion. Here, we are asking if upon aptamer-target binding, will there be a change in diffusion across a 
microcapsule wall. Once again, the target sulforhodamine B will be used as our fluorescent reporter molecule and the sulforhodamine B aptamer will be used as our model aptamer system. It is important to note that the calculated diffusion coefficients in this chapter are solely for this dye and aptamer pair and will vary for different aptamer-target pairs due to various reasons, including the molecular weights of the targets, size of the aptamers and their subsequent secondary structures formed upon binding.

\subsection{Experimental}

\subsubsection{Materials}

Calcium Chloride dihydrate $\left(\mathrm{CaCl}_{2} \bullet\left(\mathrm{H}_{2} \mathrm{O}\right)_{2} ;>99 \%\right)$, sodium carbonate $\left(\mathrm{Na}_{2} \mathrm{CO}_{3} ;>99.5 \%\right)$, ethylenediaminetetraacetic acid (EDTA; 99\%), poly(diallyldimethylammonium chloride) (PDDA; $\left.\mathrm{M}_{w} \sim 100,000-200,000 \mathrm{Da}\right)$, poly(allylamine hydrochloride) (PAH; $\left.\mathrm{M}_{w} \sim 56,000 \mathrm{Da}\right)$, and poly(styrenesulfonate) (PSS; $M_{w} \sim 100,0000 a$ ) were purchased from Sigma-Aldrich and used as received. The fluorescein modified and unmodified oligonucleotides for the sulforhodamine B aptamer (SA) and random oligonucleotide (RO) were synthesized on a MerMade 6 (Bioautomation corporation) using standard phosphoramidite chemistry ${ }^{138}$. All other reagents were of technical grade and used without further purification. All glassware was rinsed $5 X$ with distilled and $5 X$ with deionized water). 


\subsubsection{Methods}

Calcium carbonate spheres: Calcium carbonate $\left(\mathrm{CaCO}_{3}\right)$ spheres were synthesized by methods described elsewhere ${ }^{139}$. In brief, $200 \mathrm{mg}$ of PSS added to $50 \mathrm{~mL}$ of $0.33 \mathrm{M} \mathrm{CaCl}_{2}$ (in deionized water) was mixed with an equal volume of $0.33 \mathrm{M} \mathrm{Na}_{2} \mathrm{CO}_{3}$ under rapid stirring (1100rpm) with a magnetic stir bar for 1 minute in a $2 \mathrm{~L}$ beaker. The precipitate was allowed to sit in solution for 10 minutes and then filtered under vacuum and air dried overnight. SEM was performed to confirm morphology in dry state, while confocal microscopy was performed on wet samples to confirm stability. No additional characterization was performed.

Polyelectrolyte microcapsules: The $\mathrm{CaCO}_{3}$ spheres were coated by adding $1 \mathrm{~mL}$ of $2 \mathrm{mg} / \mathrm{mL}$ cationic polyelectrolyte (PAH or PDDA) in $0.5 \mathrm{M} \mathrm{NaCl}$ to $\sim 10 \mathrm{mg}$ of $\mathrm{CaCO}_{3}$ spheres. The spheres were gently shaken for 10 minutes after which they were centrifuged at $1.6 \mathrm{xg}$ for 2 minutes. The supernatant was removed and the spheres were resuspended in $1 \mathrm{~mL}$ of water and centrifuged under the same conditions as above for a total of 5 washings per layer addition. The anionic polymer, $1 \mathrm{~mL}$ of $2 \mathrm{mg} / \mathrm{mL}$ poly(sodium 4-styrene-sulfonate) (PSS, $\mathrm{Mw} \sim 100,000)$ in $0.5 \mathrm{M} \mathrm{NaCl}$, and oligonucleotides ( $\sim 0.3 \mu \mathrm{M}$ in pH 7.3 Tris buffer) were deposited in alternating layers with PAH or PDDA in the same manner as described above. The cores were removed by adding $1 \mathrm{~mL}$ of 500mM EDTA in $500 \mathrm{mM} \mathrm{pH} 7.3$ Tris buffer and stirring gently overnight. This was repeated $3 X$ to ensure complete removal of $\mathrm{CaCO}_{3}$ cores. The microcapsules were isolated by centrifugation at $0.6 \times g$ for 1 minute and washed with a total volume of $5 \mathrm{~mL}$ of water to remove 
remaining EDTA as monitored by a $\mathrm{pH}$ change to $\sim 7$. The microcapsules were stored suspended in water or $\mathrm{pH} 7.3$ Tris buffer and found to be stable for at least 2 weeks. For the test with the sulforhodamine $\mathrm{B}$ dye, the microcapsules (annealed at $70^{\circ} \mathrm{C}$ for 10 minutes and unannealed) were mixed with $10 \mu \mathrm{M}$ solution of dye for half an hour before deposition on the microscope slide.

Microscopy: All images were taken at RT. For the SEM, wet samples were placed on a clean glass microscope slide and dried by leaving in air overnight. SEM was performed on gold/palladium coated samples on a JEOL JSM-6400. Samples for confocal microscopy (Zeiss LSM510) were prepared by placing $10 \mu \mathrm{L}$ of solution (neutral pH; suspended microcapsules from a $1 \mathrm{mg} / \mathrm{mL}$ stock solution) on a clean microscope slide and placing a cover slip on top. We found that placing nail polish and letting it dry on the corners of the cover slip kept the cover slip elevated and kept the microcapsules from being crushed, thereby allowing us to image the samples wet. The excitation lasers used were $489 \mathrm{~nm}$ ( $20 \%$ laser intensity for fluorescein) and/or $561 \mathrm{~nm}(0.5-20 \%$ laser intensity for sulforhodamine B). Both the fluorescence and light channels were monitored during the studies for image sharpness and accuracy.

Colocalization: Colocalization studies were performed on wet $(\mathrm{PAH})_{4}(\mathrm{SA})_{2}(\mathrm{PSS})_{2}{ }^{\mathrm{a}}$ where the SA was tagged by fluorescein at the $5^{\prime}$ end. To allow for folding of SA, the microcapsules were heated at $70^{\circ} \mathrm{C}$ for 10 minutes and cooled back down to room temperature before imaging. A minimum of four microcapsules per sample (with and

\footnotetext{
The build-up of the films was: PAH/PSS x 1, PAH/DNA $\times 2$, PAH/PSS $\times 1$
} 
without $0.1 \mathrm{M} \mathrm{K}^{+}$) were imaged simultaneously with the $489 \mathrm{~nm}$ and $561 \mathrm{~nm}$ lasers to measure fluorescence from the fluorescein (tag) and sulforhodamine B (target, 10 $\mu \mathrm{M}$ ) fluorophores, respectively. The two fluorescence signals in each sample were carefully aligned using the in-house Zeiss software to account for any drift during the image capture.

Fluorescence Recovery After Photobleaching (FRAP): These samples were not labeled with fluorescein to avoid any overlapping emissions with the sulforhodamine B emission during FRAP. An average of 8 microcapsules per sample were used for FRAP studies using $10 \mu \mathrm{M}$ sulforhodamine, under wet conditions $(0.1 \mathrm{M} \mathrm{KCl})$. Annealed samples (SA and RO) were heated at $70^{\circ} \mathrm{C}$ for 10 minutes and cooled back down to room temperature prior to imaging. The samples were bleached at various laser intensities with the 561nm laser depending on the amount of dye inside the microcapsule and the recovery was collected at $0.5-12 \%$ laser intensity at 1 second intervals. Microcapsules whose cores were not completely dissolved and showed total fluorescence recoveries of less than $80 \%$ were not included. The fitting was done using a normalized first order fit, with the kinetics software for the Zeiss microscope. Diffusion coefficients $(D)$ in $\mu \mathrm{m}^{2} / \mathrm{s}$ were calculated using Equation 3.

\subsection{Results and Discussion}

We have previously shown that aptamers retain their sensitivity and specificity in bilayers films using QCM, CLSM, and ToF-SIMS. In this chapter, we wanted to study 
aptamer microcapsules in an effort to understand the permeability of such films upon aptamer-target binding. Such systems would not only help in the development of our smart fertilizers, but it can be extrapolated to any use where smart release is required, including drug delivery. Specifically, we wanted to (1) show successful formation of aptamer-embedded microcapsules; (2) test if specificity of the aptamer-target interaction is retained in spherical type morphology; and if so (3) can the diffusion be altered upon aptamer-target binding.

It is important to emphasize, at this point, the key difference between our work and other work with DNA microcapsule-systems. There are several examples of polyelectrolyte systems for controlled DNA delivery where DNA is either found within the capsule itself or comprises part of the capsule coating ${ }^{140}$. In the current work, we are not endeavoring to deliver DNA as the cargo, rather we are using selective binding DNAs (aptamers) within the microcapsule walls to release a cargo in the presence of a molecular stimulus.

\subsubsection{Calcium carbonate spheres}

We have used microcapsules, in contrast to nanocapsules, due to the ease of detection on the CLSM (resolution ca. $2 \mu \mathrm{m}$ ). To form microcapsules, we need a sacrificial spherical template which can be easily removed to form microcapsules and not disrupt the behavior of our aptamer (such as, degradation, denaturing, $\mathrm{H}$-bond disruption). The formation of microcapsules on sacrificial cores is not novel. Various cores have been used, including silica, requiring hydrofluoric acid to be removed ${ }^{141}$ and melamine 
formaldehyde, requiring an acidic $\mathrm{pH}$ (typically hydrochloric acid) ${ }^{142}$. In contrast, we needed a core which could be removed without being potentially damaging to our aptamer (e.g. one that does not require significantly lowering the $\mathrm{pH}$ ). Calcium Carbonate $\left(\mathrm{CaCO}_{3}\right)$ has been used extensively to form microcapsules of various films, because removal of the core requires only $\mathrm{Ca}^{2+}$ chelation by EDTA ${ }^{143}$. Various research groups have used the anionic charge on $\mathrm{CaCO}_{3}$ cores to template $\mathrm{Ibl}$ films ${ }^{144}$, anchor fluorescent sensors on the surface ${ }^{145}$, template cross-linked DNA ${ }^{146}$, etc. Using standard precipitation pathways to produce $\mathrm{CaCO}_{3}$ from the respective salts of calcium and carbonate yields a cubic structure, which is the thermodynamically preferred crystal form at equilibrium ${ }^{147}$. During our synthesis of the cores, we found predominant cubic structures and very little spherical cores, which are needed for the microcapsules. All the literature surveyed on the formation of the cores, seemed to omit subtle details to the precipitation synthesis to form predominantly spheres. In some cases, groups formed only a small percentage of $\mathrm{CaCO}_{3}$ spheres, which met the objectives of their projects. Our cubic $\mathrm{CaCO}_{3}$ cores are shown in Figure 3.2(a). To produce predominantly spheres, we increased the stirring speed and time (Figure 3.2(b)). We believe that this forced spherical precipitation and prevented cubic structures from being formed as the solution was highly turbid and could not achieve a thermodynamically stable state (reach equilibrium). The cores were stable for $>2$ years. 


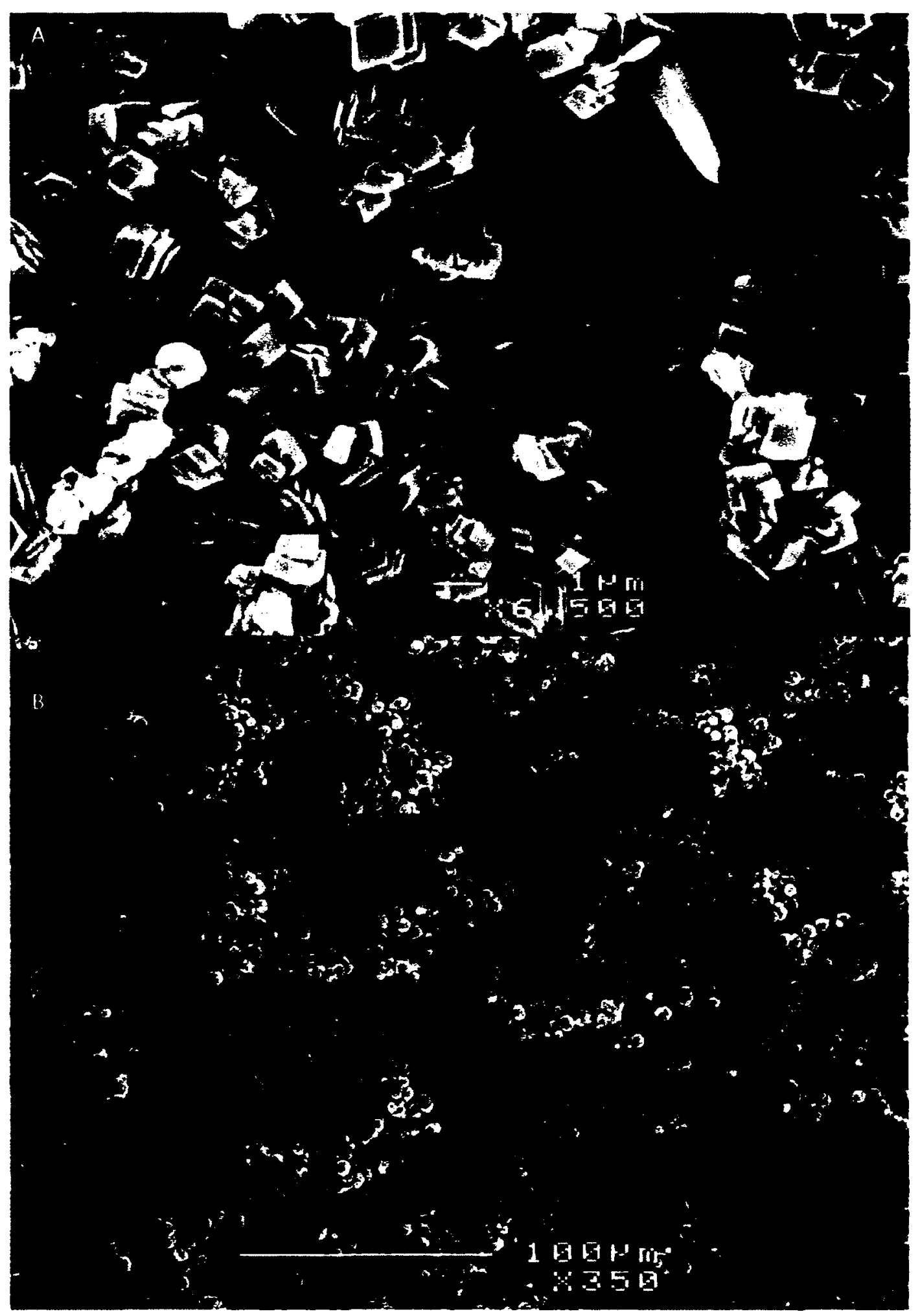

Figure 3.2: SEM micrographs of PSS doped $\mathrm{CaCO}_{3}(\mathrm{a})$ cubes; and (b) spheres. The spheres were formed under very high stirring (high shearing) and a longer stirring time. 


\subsubsection{Microcapsules}

We formed Ibl films by the methodology already described in Chapter 2 . Instead of rinsing the bilayer films in the previous chapter, we removed excess polyelectrolyte by centrifuging the cores and removing the supernatant and by following the same procedure with deionized water. We found that high centrifugation caused the $\mid \mathrm{bl}$ template cores to stick, subsequently terminating the Ibl process. We also found that once coated, the cores had a tendency to stick to the side walls of the $1.5 \mathrm{~mL}$ centrifuge tubes. We prevented this by transferring the solution into a new $1.5 \mathrm{~mL}$ tube after mixing with polyelectrolyte. Templating the cores using PDDA and PSS, and subsequent removal of cores using EDTA, we were unable to form microcapsules (Figure 3.3).

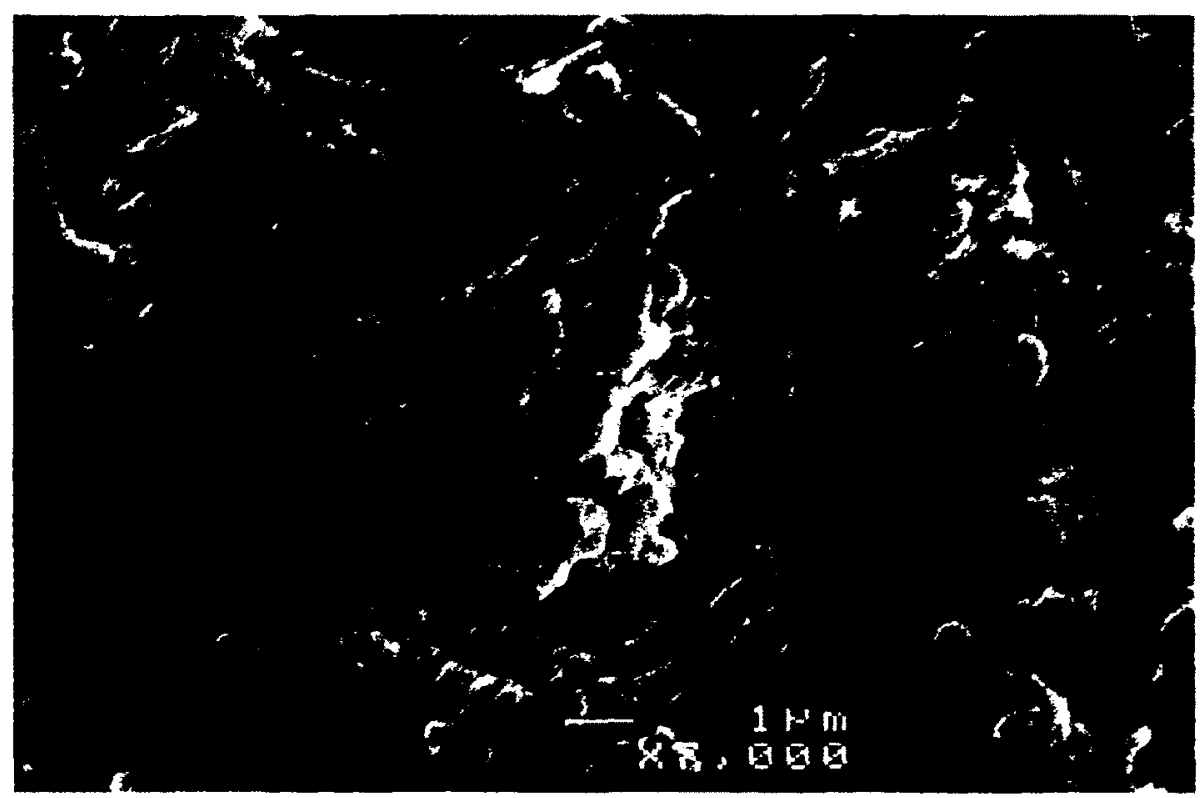

Figure 3.3: Figure : SEM micrograph of PDDA/PSS IbI films after core dissolution. SEM performed on a gold/palladium coated sample. There was no presence of microcapsules in the sample. 
Various groups have commented on the effect of osmotic pressure as the core is removed, which causes the microcapsule to collapse ${ }^{148}$. An extensive review by Lars and co-workers found that the temporarily high concentration of ions upon dissolution of the core generates an osmotic pressure relative to the outside and can lead to wall breach and subsequent destruction of the microcapsules ${ }^{149}$. They found this to be the case for microcapsules containing PDDA cationic layers. We believe osmotic pressure to be reason for the collapsed microcapsules seen in Figure 3.6. To reduce this affect, groups have used polymers as scaffolds to hold the microcapsules in place by providing rigidity. Tong et. al. ${ }^{150}$ doped the aqueous solution during precipitation of $\mathrm{CaCO}_{3}$ with PSS which gets encapsulated in the spherical cores. This allowed them to produce stable microcapsules of $\sim 8 \mu \mathrm{m}$ diameters. Previous work on the stimuli-sensitive permeability of similarly prepared polyelectrolyte microcapsules have confirmed that the inclusion of PSS in the core does not impede capsule formation and that this is a valid approach for preparing microcapsules for this type of study ${ }^{151}$. However, using PSS-doping, we were still unable to form microcapsules of PDDA and PSS (Figure 3.4). 


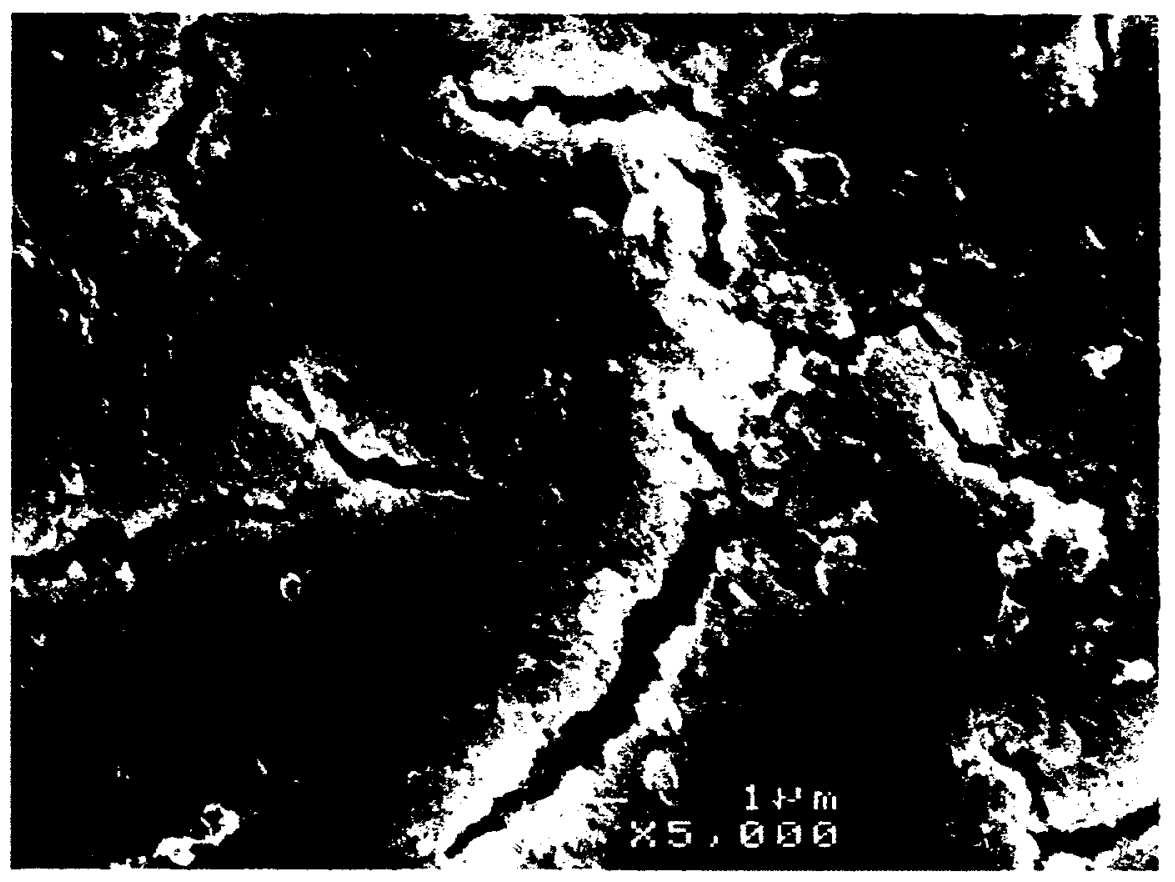

Figure 3.4: SEM micrograph of Ibl films template on a PSS doped $\mathrm{CaCO}_{3}$ cores with $(\mathrm{PDDA})_{4}(\mathrm{PSS})_{4}$. No microcapsules were seen in the micrographs.

We decided to move to a more rigid and less swollen (in aqueous solution) polymer PAH. PAH, in contrast to PDDA has a much smaller size, thus, likely has higher rigidity. PDDA is also known to highly swell in aqueous solutions, likely because of $\mathrm{H}$-bonding between the highly charged ammonium (in the backbone) and water ${ }^{152}$. PAH/PSS microcapsules have been used extensively in literature ${ }^{153}$. Using PSS doped $\mathrm{CaCO}_{3}$ cores, we were able to form PAH/PSS microcapsules which were stable in solution. Figure 3.5 (a) shows SEM micrographs of the microcapsules once dry. The Ibl film can be seen on the outside of the microcapsule. The average size of the microcapsules dry is ca. 2-4 $\mu \mathrm{m}$. Shown in Figure $3.5(\mathrm{~b})$ and $(\mathrm{c})$ is the CLSM images of the microcapsules, where green represents fluorescence from the fluorescein tagged aptamer (fluorescence just in 
the microcapsule wall) and yellow/orange represents fluorescence from the target (sulforhodamine B), i.e., where the microcapsule is filled with target dye and there is a constant flux moving through the microcapsules at all times. The CLSM images were taken in solution, and show an average size of ca. $8 \mu \mathrm{m}$ which is in agreement with literature. 

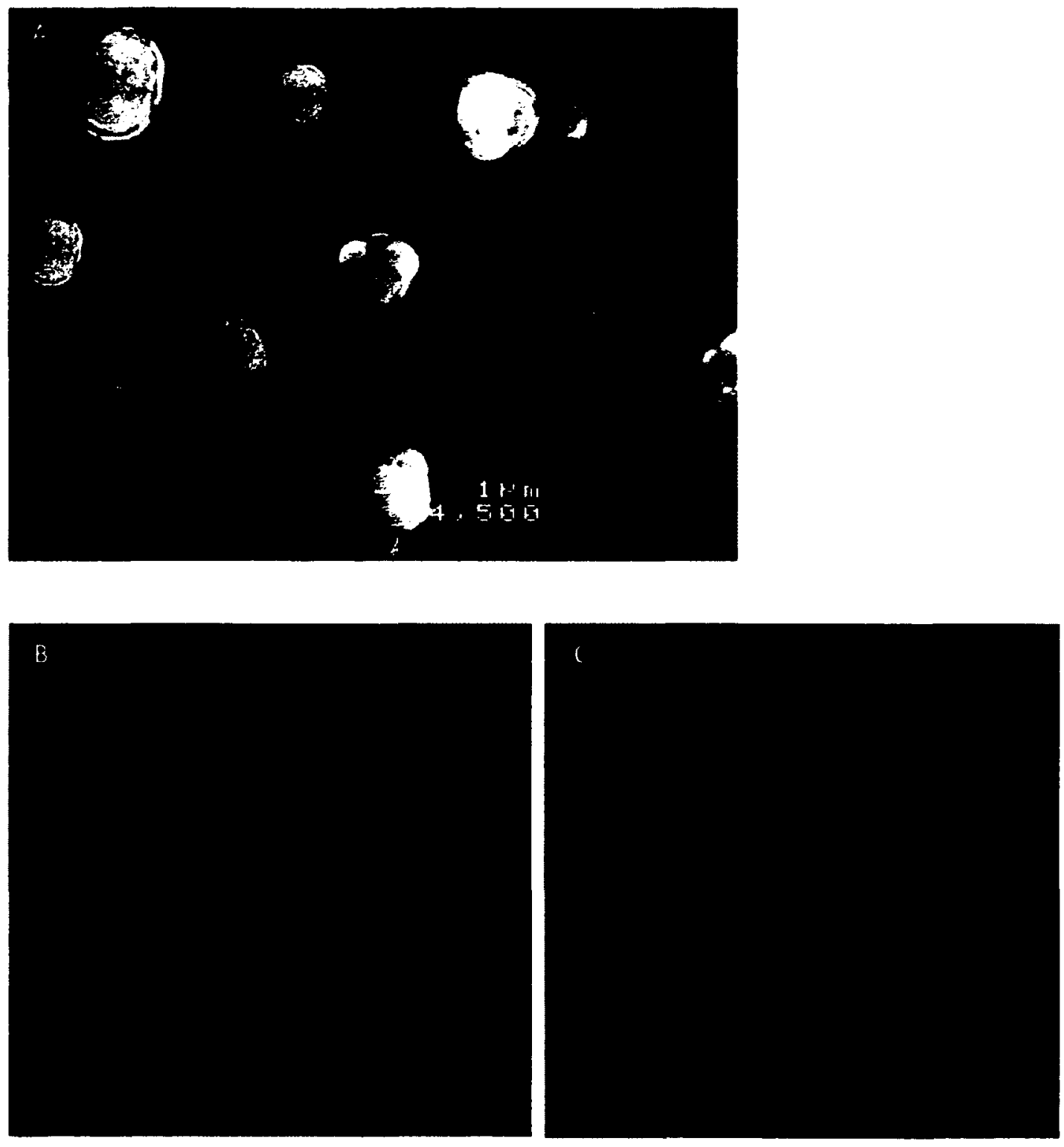

Figure 3.5: (a) SEM micrograph of PSS doped PAH/PSS-aptamer microcapsules $(\mathrm{PAH})_{4}(\mathrm{SA})_{2}(\mathrm{PSS})_{2}$ with an average size of ca. $2 \mu \mathrm{m}$ [dry]; (b) CLSM of fluorescein labeled aptamer microcapsules $(\mathrm{PAH})_{4}(\mathrm{SA})_{2}(\mathrm{PSS})_{2}$ (left, $489 \mathrm{~nm}$ laser); and (c) unlabelled sulforhodamine $B$ filled microcapsules $(\mathrm{PAH})_{4}(\mathrm{SA})_{2}(\mathrm{PSS})_{2}$ (right, $561 \mathrm{~nm}$ laser) [wet]. Microcapsules in CLSM study had an average size of ca. $8 \mu \mathrm{m}$. 


\subsubsection{Co-localization}

After formation, the microcapsules were tested to ensure aptamer specificity. We have already shown in Chapter 2 quantitatively by ToF-SIMS and by determination of a $K_{d}$ that aptamers in bilayer films retain their binding affinity. In lieu of that, we considered it sufficient to prove semi-quantitatively using CLSM that that specificity holds true for microcapsules as well. This was done using colocalization on the CLSM. Colocalization experiments are routinely used to study spatial arrangements of different biological systems by staining and imaging different molecules ${ }^{154}$. We tagged our aptamer with fluorescein (green) so we could image its spatial arrangement and used the sulforhodamine B fluorescence (orange) to image the spatial arrangement of the target. The experiment was to concurrently (1ns delay) image the fluorescein and sulforhodamine B channels, overlay the images and obtain a spatial intensity subtraction. The presence of a resultant fluorescein signal (green color) would suggest the absence of specific binding, while complete removal of the signal would indicate specificity. Colocalization was studied in the presence and absence (negative control) of $\mathrm{K}^{+}$ions which are needed to stabilize this aptamer's G-quadruplex secondary structure upon target binding. To ensure real data, we first needed to ensure that (a) there was no background fluorescence from $\mathrm{CaCO}_{3}$ cores (Figure 3.6(a) in case there was any presence of remaining $\mathrm{CaCO}_{3}$; and (b) there was no emission overlap between signals from the fluorescein and sulforhodamine B specific lasers (Figure 3.6(b)). To remove emission overlap, we needed to fine tune the diameters of the filters to ensure that signal collection was at a region of no overlap. To do this, we used a graphical 
demonstration program built into the microscope software which allows for testing of potential emission overlaps of different dyes. After optimization of the filters, it can be seen (Figure 3.6(b)) that there is no overlap intensity from the green (fluorescein) and orange (sulforhodamine B) lasers expected, which would show on the screen as a combination of the two colors upon intensity addition and which would be flagged by the microscope software. 

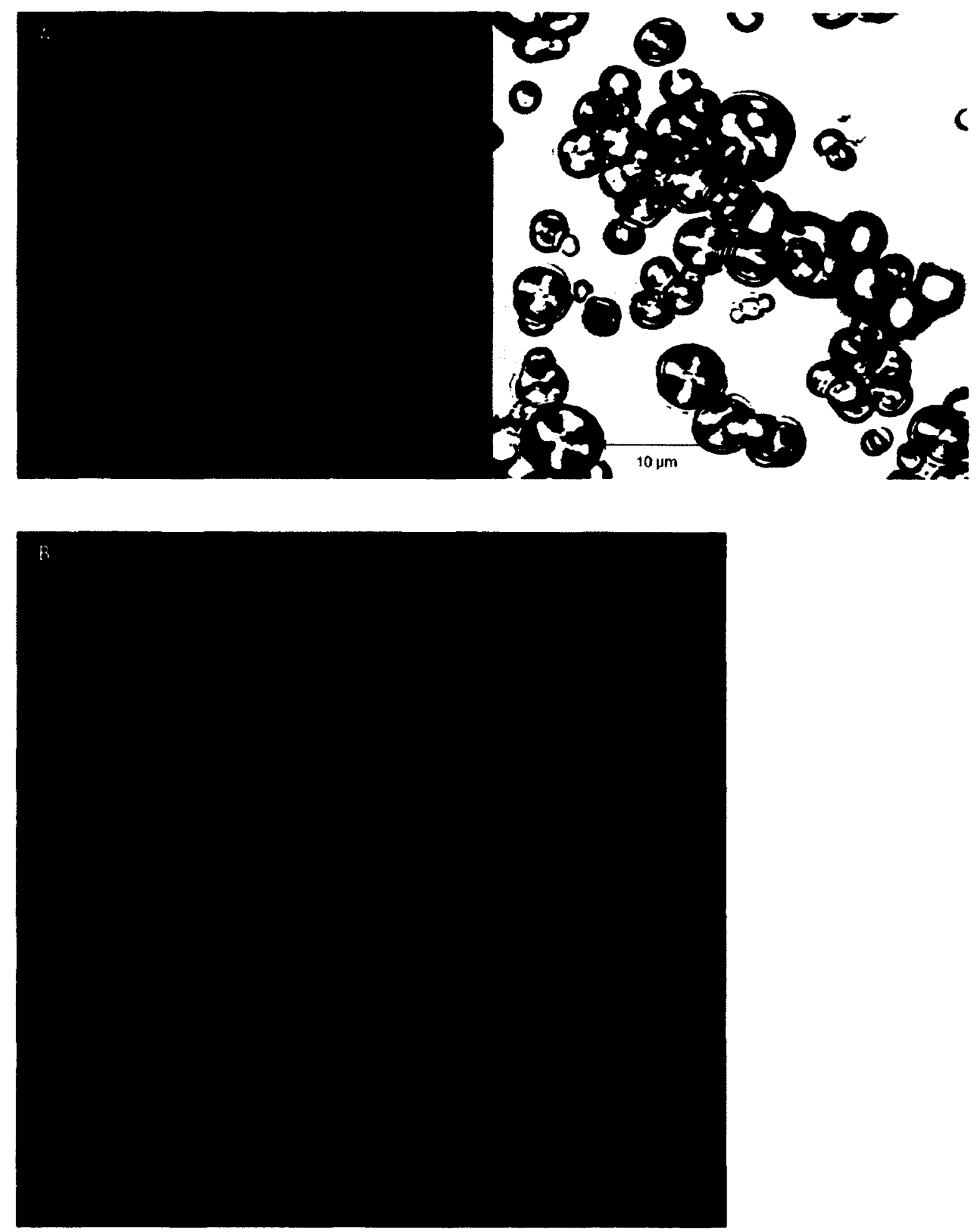

Figure 3.6: (a) CLSM image of $\mathrm{CaCO}_{3}$ cores shown in the light channel (right), indicating lack of fluorescence (fluorescence channel, left); and (b) theoretical modeling after careful alignment of CLSM optics to avoid any overlap from the two emissions of the fluorophores. 
Fluorescence colocalization experiments were performed with the sulforhodamine B dye and SA microcapsules in the absence and presence of monovalent $K^{+}$which is needed for g-quadruplex folding (Figure 3.7). Fluorescein labelled-SA was once again used for these experiments. CLSM images were taken using two different laser excitation sources to monitor the presence and location of the two species (green color denotes fluorescein emission, orange indicates sulforhodamine B emission, yellow is the result of colocalized emission from both the aptamer and its target). Figure 3.7 a-c shows the results of the $\mathrm{K}^{+}$-free experiment. The presence of green fluorescence from the aptamer within the microcapsule walls is visible in the green channel (Figure 3.7(a)) and a small amount of the target's fluorescence can be seen in the orange channel (Figure 3.7(b)). However, when the two channels are overlaid (Figure 3.7(c)), it appears that the majority of the green and red signal does not overlap, as evidenced by very little yellow (colocalized) emission. This corresponds well with what was observed in our previous work, where the polyelectrolyte films displayed only a low level of nonspecific binding with the sulforhodamine B target in the absence of $\mathrm{K}^{+}$. In contrast, the images from experiments run with the same concentration of target (using the same optical setup), but also in the presence of $\mathrm{K}^{+}$(Figure $3.7(\mathrm{~d}-\mathrm{f})$ ) show a different result. Once again, the aptamer and target emission can be seen in the green and orange channels, respectively (Figure 3.7 (d) and (e)). However, when the channels are overlaid (Figure 3.7(f)), it can be observed that the SA is colocalized with the target as indicated by the bright yellow signal. Once again, this agrees well with our previous work on aptamer-polyelectrolyte films, and points to successful G-quadruplex formation and 
target binding in the presence of $K^{+}$. These results suggest that even within the walls of the microcapsule, the aptamer is flexible enough in the polyelectrolyte environment, allowing it to fold in the required conformation for target binding. We did colocalization experiments on 4 microcapsules per experiment. 


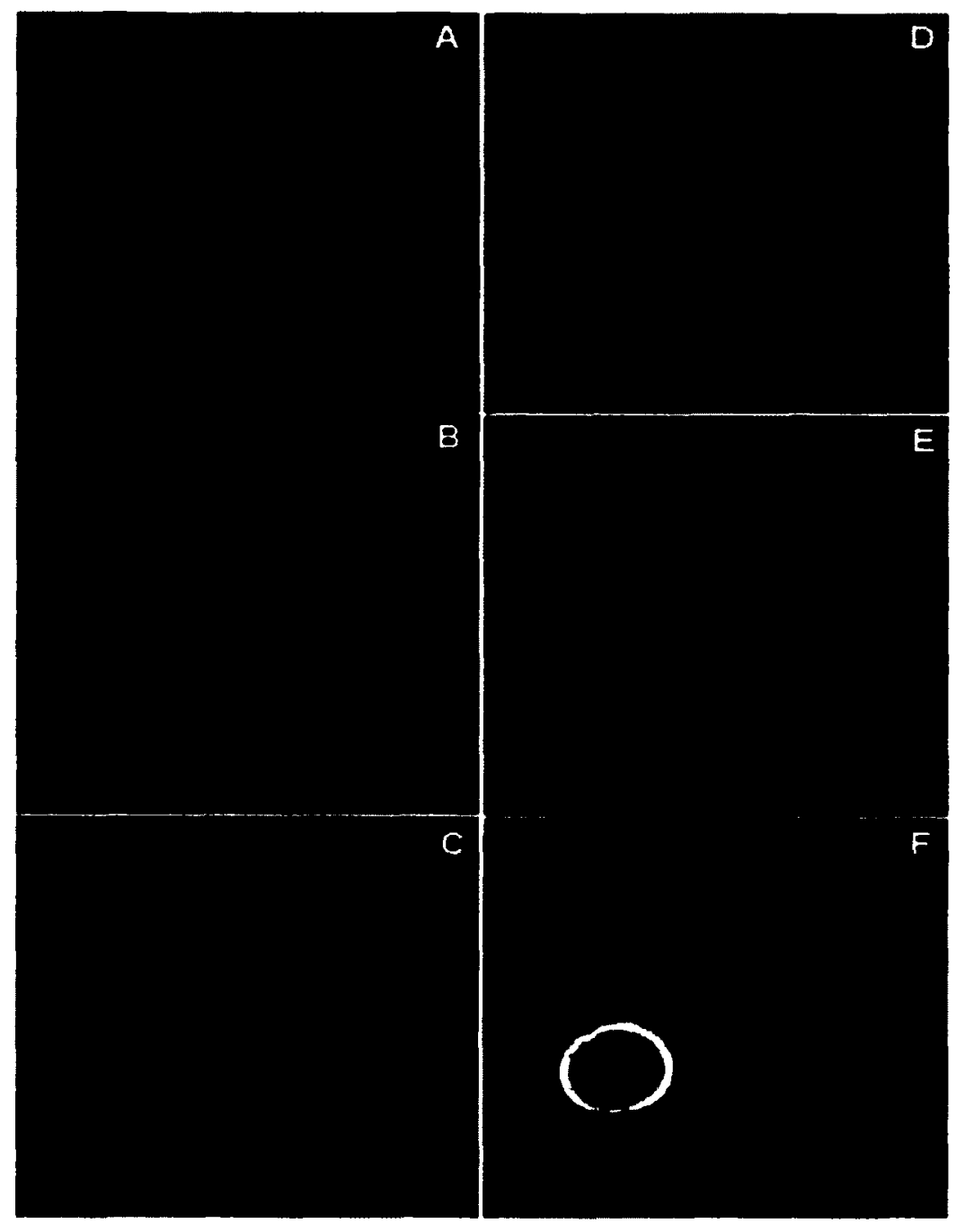

Figure 3.7: Fluorescence co-localization experiments to confirm aptamer-target binding and the requirement for $\mathrm{K}^{+}(\mathrm{a}-\mathrm{c})$ Series of binding experiments where $\mathrm{K}^{+}$was excluded from the solution; ( $d-f)$ Series of binding experiments with $K^{+}$present in solution. (A) and (D) Emission image from the green channel displaying fluorescein emission from the tagged SA within the microcapsule walls (in green); (B) and (E) Emission image from the orange channel showing the presence of the sulforhodamine $B$ target; $(C)$ and $(F)$ Overlay of orange and green channels, where yellow emission is the result of the colocalization of SA and the sulforhodamine target. It is shown that in the presence of a monovalent cation (F) there is a high degree of colocalization of the aptamer and the target, as indicated by the yellow color. In the absence of the cation, only a small degree of colocalization can be detected, while the majority of the green and red emission can be seen separately. This suggests a small degree of non-specific binding to the polyelectrolyte microcapsule occurs but that the activity of the aptamer and the majority of the binding of the target to the microcapsule requires $\mathrm{K}^{+}$. Scale bars are $10 \mu \mathrm{m}$. 


\subsubsection{Fluorescence recovery after photobleaching}

Next, we wanted to study the diffusion kinetics of the microcapsules. This was done with FRAP studies on various microcapsules containing the aptamer $(\mathrm{PAH})_{4}(\mathrm{SA})_{2}(\mathrm{PSS})_{2}$ and negative controls of $(\mathrm{PAH})_{4}(\mathrm{RO})_{2}(\mathrm{PSS})_{2}$ and $(\mathrm{PAH})_{4}(\mathrm{PSS})_{4}$. We also tested our RO and SA microcapsules before and after annealing at $70^{\circ} \mathrm{C}$ for 10 minutes and cooling to RT(see discussion in Chapter 2). We found the traditionally used concentration of EDTA $(200 \mathrm{mM})$ was insufficient to remove majority of the cores from solution. This was confirmed by FRAP, where fluorescence recovery of $<50 \%$ was considered to be due to the capsules being partially or completely filled with $\mathrm{CaCO}_{3}$ (Figure 3.8). We increased the EDTA concentration to $500 \mathrm{mM}$ (buffered) and found that it improved the core dissolutions dramatically and led to a higher percentage of microcapsules with complete

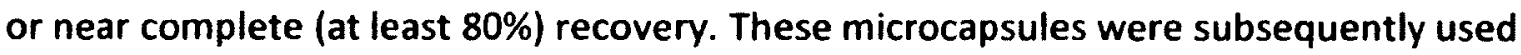
for FRAP experiments. 

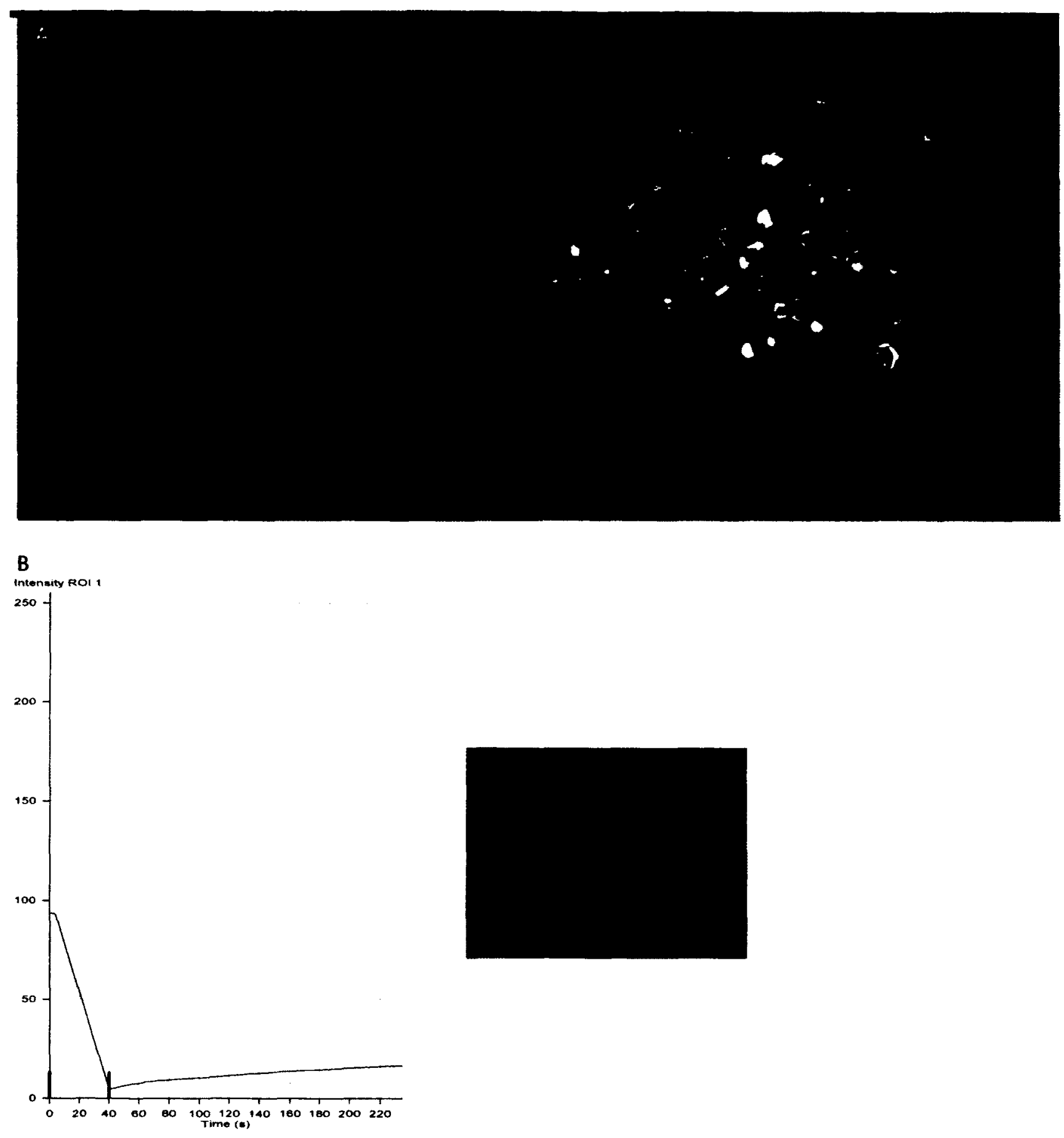

Figure 3.8: (a) CLSM image of microcapsules filled or partially filled with $\mathrm{CaCO}_{3}$ with fluorescent channel on left and light channel on right; (b) FRAP on these microcapsules shows little recovery of the target (sulforhodamine B) fluorescence intensity (left), where the red spot on the right indicates the bleach spot. The aptamer was not labeled with fluorescein in the FRAP experiments. 
In contrast, microcapsules which were not filled with $\mathrm{CaCO}_{3}$, showed a much higher recovery of fluorescence intensity (Figure $3.9(\mathrm{a})$ ). The different stages of recovery can also be seen from the picture insets in Figure 3.9 (a). The FRAP data was kinetically fit using first-order kinetics using the microscope software "Kinetic"(raw data shown in Figure $3.9(b))$. The fit allowed for a determination of rate constant $\left(s^{-1}\right)$ and $t_{1 / 2}(s)$. Using the $t_{1 / 2}$ and Equation 3, we were able to calculate the Diffusion coefficient for the dye permeation through the walls of our microcapsules. 
A 300
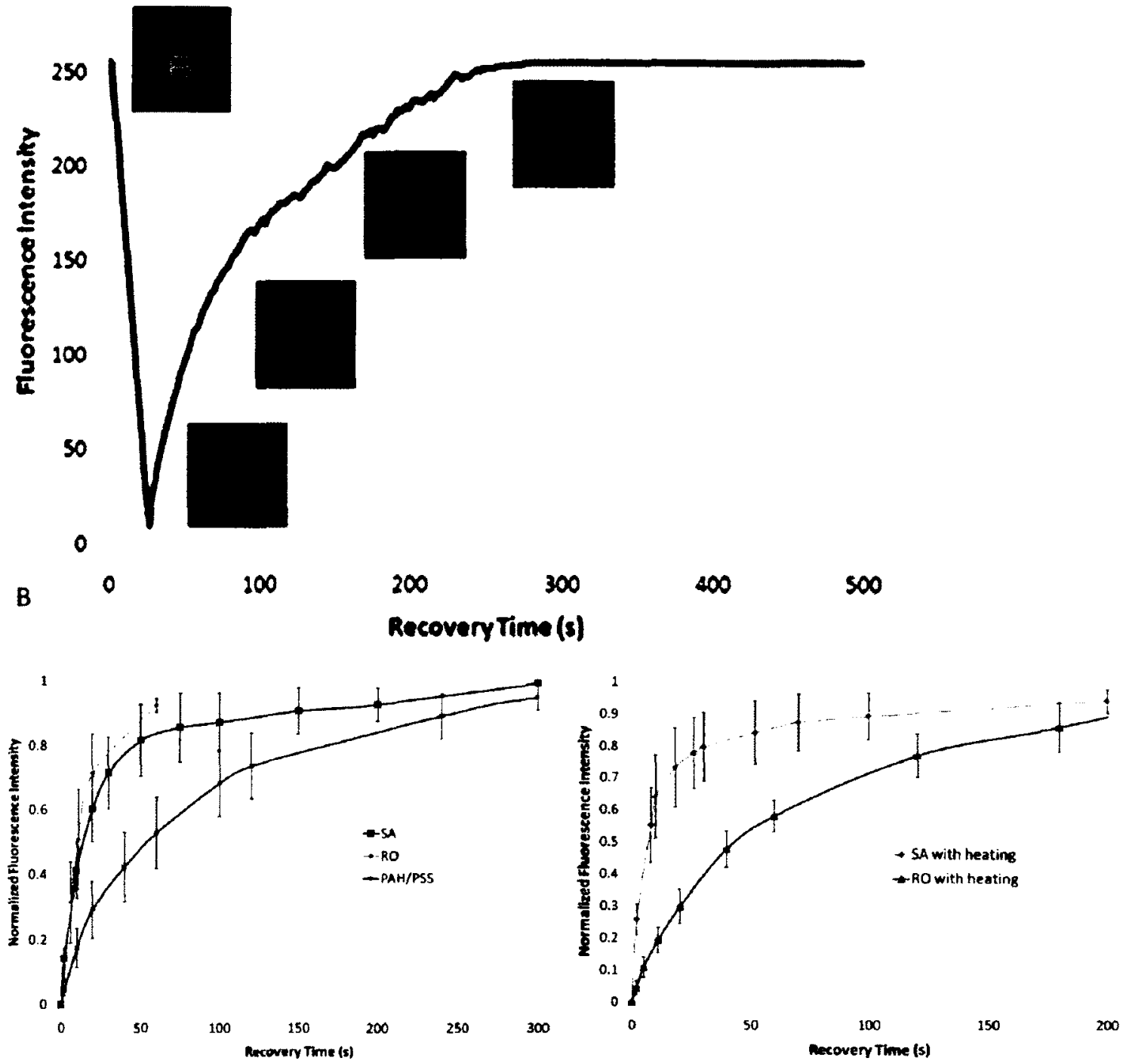

Figure 3.9: (a) FRAP of microcapsules without $\mathrm{CaCO}_{3}$, showing the photobleaching and measurement of the subsequent fluorescence recovery in seconds; and (b) raw FRAP data after first-order kinetics fit using the microscope software 'Kinetic'. The $t_{1 / 2}$ from the fit data was used to calculate the diffusion coefficient. Figure $(b)$ is not corrected for bleach spot size and is presented here to show a relative change between the three types of microcapsule conditions tested. 
The calculated diffusion coefficients are shown in Table 3-1:

Table 3-1: Diffusion Coefficients obtained by FRAP experiments on the microcapsules

\begin{tabular}{ll}
\hline Microcapsule composition & Diffusion coefficient $\left(\mathrm{m}^{2} / \mathrm{s}\right)$ \\
\hline PAH/PSS & $2.9( \pm 1.9) \times 10^{-15}$ \\
RO before annealing & $3.1( \pm 0.4) \times 10^{-15}$ \\
SA before annealing & $5.5( \pm 2.5) \times 10^{-15}$ \\
RO after annealing & $2.4( \pm 0.7) \times 10^{-15}$ \\
SA after annealing & $19( \pm 11) \times 10^{-15}$ \\
\hline
\end{tabular}

We found that compared to the two negative controls (random strand RO and just PAH/PSS), our SA microcapsules have ca. 10X higher permeability. While capsule to capsule variability was sizeable in some samples, our reported diffusion coefficient for the PAH/PSS microcapsule is in good agreement with literature data on PAH/PSS microcapsules at similar ionic strength $\left(\sim 1 \times 10^{-15} \mathrm{~m}^{2} / \mathrm{s}\right)^{155}$. As known from our previous work (Chapter 2), annealing of the polyelectrolyte-SA coatings in the presence of $\mathrm{K}^{+}$ions ensures the complete folding of the aptamer into its characteristic g-quadruplex conformation, a requirement for efficient target binding. Thus, annealing is expected to improve sulforhodamine B diffusion through the capsule. In contrast, a study on the effect of annealing on PAH/PSS microcapsule permeability found that diffusion coefficients are reduced upon heat treatment ${ }^{156}$.This was attributed to the repair of charge pairing defects that could be present in the capsule walls after the dissolution of 
the core. Interestingly, heat annealing had almost no effect on the RO microcapsules and led to an increase the diffusion coefficient by $30 \%$ for the SA microcapsules. This leads to almost an order of magnitude difference in sulforhodamine diffusion coefficient between the annealed RO controls and the annealed SA samples.

These data suggest that defects in polyelectrolyte layers containing oligonucleotides may be more difficult to repair, but, more importantly, any decrease in permeability resulting from defect repair is overshadowed by the increase in permeability due to the improvement of aptamer folding after annealing. In other words, the aptamer is better able to bind the target, leading to an increase in permeability. Two possible scenarios could explain this result. Aptamer-target binding, and any concomitant change in aptamer conformation or volume, leads to the creation of pores within the coating. These pores, in turn, increase the permeability of the microcapsule wall. Alternatively, the aptamer is acting as a site of active transport, with binding facilitating the target's movement through the microcapsule.

Examining whether the effect of aptamer-target binding on the diffusion of the target through the microcapsule is also observed for the diffusion of other molecules should help to clarify this point. Our current system using the sulforhodamine B aptamer and FRAP as a method for understanding permeability of these coatings limits our ability to attempt these experiments. The FRAP approach requires a fluorescent diffusing molecule for measuring permeability and our model aptamer's target is also fluorescent, While this has allowed us to generate our proof-of-concept data quite readily, testing 
the diffusion of other fluorescent molecules in the presence of the fluorescent target presents an experimental challenge. As such, in our next chapter we examine these coatings using electrochemistry, rather than fluorescence, to answer these questions $(\text { Chapter } 3)^{157}$.

Nevertheless, both potential mechanisms for explaining these findings may lead to the development of smart microcapsules for unique and exciting applications. Our work here suggests that aptamer-polyelectrolyte microcapsules represent a new family of stimuli-responsive materials that could be more specific than other systems based on $\mathrm{pH}$, ionic strength changes, etc. One can envision applications where these microcapsules control the release of a drug, for example, through aptamer binding to a relevant biomarker. In the current form of these microcapsules, small molecules able to diffuse into the bulk of the polyelectrolyte coating would be the ideal "triggers" for the release of the capsule's payload.

\subsection{Conclusions}

Herein we have shown that aptamers embedded within the walls of polyelectrolyte microcapsules are able to fold into their three dimensional conformation, in this case a G-quadruplex, in order to bind their associated target. Further, we have demonstrated that aptamers can be used to control the permeation of a target molecule through the microcapsule membrane. These results open up the possibility for the use of aptamer smart micro/nanostructures in fields such as medicine and agriculture. 


\section{Chapter 4. Electrochemistry}

\section{1 Introduction}

We have demonstrated that aptamers retain their binding affinity when in bilayer films (Chapter 2) and that diffusion of target molecules can be altered upon aptamer-target binding in polyelectrolyte microcapsules (Chapter 3). We next want to understand the diffusion of small molecules (other than aptamer targets) through the microcapsules upon aptamer-target binding for our smart fertilizer, i.e., to release small molecules upon aptamer-target binding. To do this, we used electrochemistry and, by measuring the peak current, we were able to calculate diffusion coefficients of our functional microcapsules. We decided to use both positively and negatively charged electroactive probes (ruthenium hexaammine and ferricyanide, respectively) to extract any chargerelated diffusion characteristics. An increased understanding of small molecule diffusion is needed because once the aptamer binds to its target in our smart fertilizer, nutrient small molecules must be able to diffuse through the microcapsule wall.

\subsubsection{Electrochemistry}

Electrochemistry encompasses the study of chemical reactions associated with charge separation. In many cases, this charge separation will be followed by some form of charge transfer in solution close to an electrode surface. Charge transfer reactions, with few exceptions, lead to two or more half-reactions, in opposite directions, that is, a chemical oxidation and reduction reaction which ensure electroneutrality $y^{158}$. In an 
electrochemical cell, an electrode can behave as a source (reduction) or a drain (oxidation) of electrons present in the solution, such that

$\mathrm{O}+\mathrm{n} e^{-} \rightarrow \mathrm{R} ;$ where $\mathrm{O}=$ oxidized species; $\mathrm{R}=$ reduced species; and $\mathrm{n} e=$ number of electrons as a result of oxidation ${ }^{158}$.

In terms of energetic cost, for an oxidation there is a minimum energy needed before charge transfer can occur (i.e., a negative potential), while for reduction there is a maximum energy threshold in the electrode of the lowest unoccupied level which will accept electrons (i.e., positive potential) ${ }^{158}$. Thus, by controlling the potentials, the types, rates, and extent of an electrochemical reaction can be controlled. Faraday, who worked tirelessly to understand electrochemical processes, established two laws ${ }^{159}$ :

1. "In any electrolytic process the amount of chemical action is proportional to the quantity of electricity passing through the electrolytic conductor; and

2. The masses of different substances deposited or dissolved by the same quantity of electricity are in the proportions of their chemical equivalents."

Thus, electron or charge transfer at an electrode (solid)-electrolyte (liquid or semiliquid) interface which can yield oxidation and reduction reactions are known as Faradaic processes, while charging the interface without transfer of electrons (such as a double layer formation) is known as a non-faradaic process. An electric double layer, which is a phenomenon seen in all charged surfaces, is a dual-phase where one side carries a positive charge and the other is negatively charged. This double layer may arise 
from preferential attraction of one charge to a specific side or from the tendency of positively charged particles to move from one phase to another (or vice versa) ${ }^{159}$. Electrochemical reactions are typically measured relative to a standard electrode, such as a silver/silver chloride reference. For equilibrated half-reactions, the potential (E) can be compared to the standard electrode potential (of the reference) by the Nernst Equation:

$E=E^{\circ}-\frac{R T}{n F} \sum v_{\mathrm{i}} \ln a_{\mathrm{i}}$

Where; $v_{i}=$ stoichiometric number, $a_{i}=$ activity coefficients with assumed unit activity at equilibrium $(=1) ; R=$ ideal gas constant; $T=$ temperature $(K) ; n=$ number of electrons oxidized or reduced; $F=$ faraday constant; and $E^{0}=$ standard cell potential $(V)^{160}$.

For a system driven by thermodynamics, the potentials can be manipulated to quantify electroactive species in solution by the modified Nernst equation (equation 2), thereby showing that the final potential $(E)$ varies with the logarithmic ratio of [oxidized species]/[reduced species].

$E=E^{\circ}+\frac{2.3 R T}{n F} \log \left(C_{\mathrm{o}}(0, t) / C_{\mathrm{R}}(0, t)\right.$

Where $C_{o}$ and $C_{R}$ are the concentrations of electroactive species oxidized and reduced $^{161}$

The route for the electroactive species to reach the electrode surface from solution can involve multiple different processes. In a simplistic situation, mass transport from the 
bulk solution to the electrode surface, electron transfer across the interface, and transport of products formed back to the bulk, drive an electrochemical reaction, with the mass transport being the rate limiting step. When the overall reaction is controlled (limited) only by mass transfer of the bulk species to the electrode surface, the reaction is said to be mass-transport limited. These reactions are called reversible or Nernstian as they obey thermodynamic laws according to Equation $1^{161}$. Mass transfer can be limited or controlled by three primary steps: (1) Diffusion - movement by concentration gradient; (2) Migration - movement by electrical field for charged particles and (3) Convection - movement by physical movement such as stirring (see Figure 4.1 below).

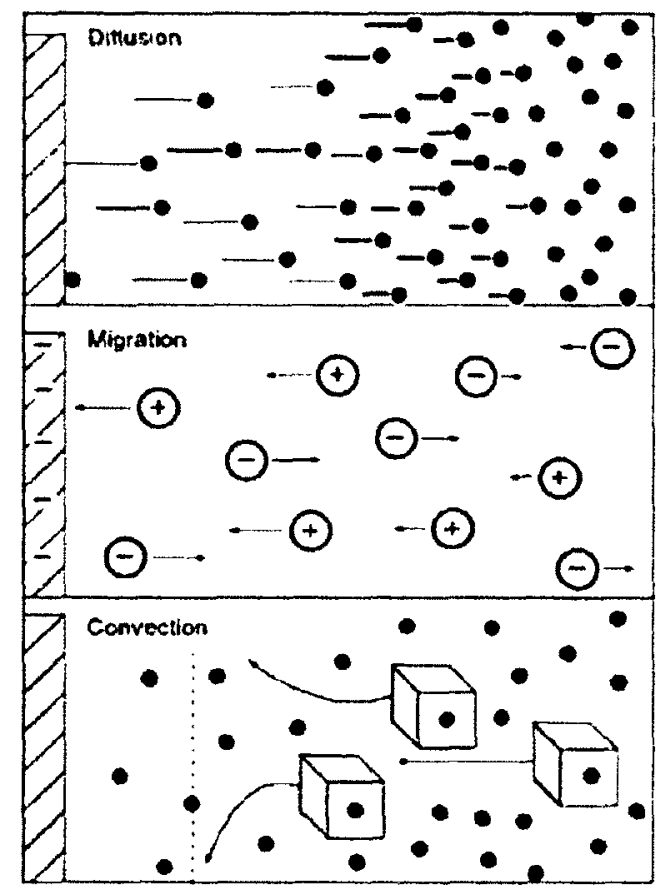

Figure 4.1: Mechanisms of mass-transport limited systems: (a) diffusion limited where ions are governed only by diffusion and not charge attraction; (b) migration limited transport showing attraction of an opposite charge (in this case positive) to the electrode and electrostatic repulsion of the like charge (negative); and (c) convection limited where ion mobility is promoted by physical means such as stirring or shaking ${ }^{161}$. 
To quantify these mechanisms for a planar or spherical electrode, the current (1) can be calculated from the semi-infinite linear diffusion:

$I=n F A D\left(\frac{\partial c}{\partial x}\right)$

Where $I=n F A J ; \mathrm{J}=$ flux; $\mathrm{x}=$ distance from the electrode and $\mathrm{c}=$ concentration at either electrode.

Solving for Ficks second law (equation 4) and simplifying with a linear transformation, the commonly used Cottrell equation ${ }^{162}$ can be determined (equation 5).

$\frac{\partial c}{\partial t}=D \frac{\partial^{2} c}{\partial x^{2}}$

Where $D=$ diffusion over time $(t)$

$I(t)=\frac{n F A D^{1 / 2} c_{\infty}}{(\pi t)^{1 / 2}}$

Where, the concentration of the electroactive species is assumed to be infinite in the bulk solution $\left(c_{\infty}\right)$.

Therefore, by studying the change in current, and knowing the surface area of the electrode, one can extrapolate diffusion which will be a combination of diffusion, migration and convection. 


\subsubsection{Electrodes \\ Working Electrodes}

Working electrodes used are typically characterized into metals, carbon, mercury, and other solid materials. Metal based working electrodes (such as gold and platinum) are used due to their high conductivity which yields very low background current. Their sensitivity can be increased by physical methods (such as stirring), which increases diffusion across the bulk solution and the double layer to the electrode. The surface of metal electrodes can be easily modified and that is why they are heavily utilized in experimental studies. For example, gold electrodes can be easily modified with thiolfunctional compounds ${ }^{163}$. Another advantage of using metal electrodes is their ease of polishing. Typically, one can completely clean a metal working electrode with polishing and an acid etch. However, the major drawback of these electrodes is corrosion or passivation over time which limits their prolonged use ${ }^{164}$.

\section{Reference and Auxiliary electrodes}

The auxiliary electrode generally made of platinum (or other relatively inert conductive metals) receives current from the working electrode to complete the electrochemical cell. It has a larger area than the working electrode to receive current, thereby, ensuring only current changes in the working electrode are measured. Historically, the auxiliary electrode was also used as a reference electrode, which compares the measured potential against a standard. However, it was found that the chemical activities of the auxiliary electrode could be changed due to passing current (nonspecific reduction- 
oxidation reactions) and would shift the reference potential. To overcome this, a 3-cell system is now commonly (if not always) used where a third electrode has only a reference function for the cell. Typical reference electrodes used include standard calomel electrode $(S C E ; E=+0.242 \mathrm{~V}$ vs. $S H E)$, and silver chloride electrode $(E=+0.197 \mathrm{~V}$ vs. SHE). Thus, to find the current on the working electrode, the reading is compared against the reference to solve for the Nernst equation (Equation (1)). In most cases, the reference electrode is separated by a porous frit to avoid contamination (poisoning) from the reactions occurring at the working electrode. Further, to make accurate measurements on the working electrode, it is important to ensure that there is little resistance between the reference and working electrode, meaning that they need to be in close proximity to each other (see Figure 4.2). Groups have also found that ensuring equidistance between the three electrodes during measurements helps in the reproducibility of results. In this project, we used a 3-electrode cell with a gold working electrode, silver chloride reference electrode and platinum auxiliary electrode as shown in Figure 4.2. The system was purged with argon to remove dissolved oxygen $(E=1.27 \mathrm{~V}$ vs. SHE), however, any changes to the current due to dissolved $\mathrm{O}_{2}$ will be minimal as the electroactive probes used in this project have redox potentials much lower than that of dissolved $\mathrm{O}_{2}$. 


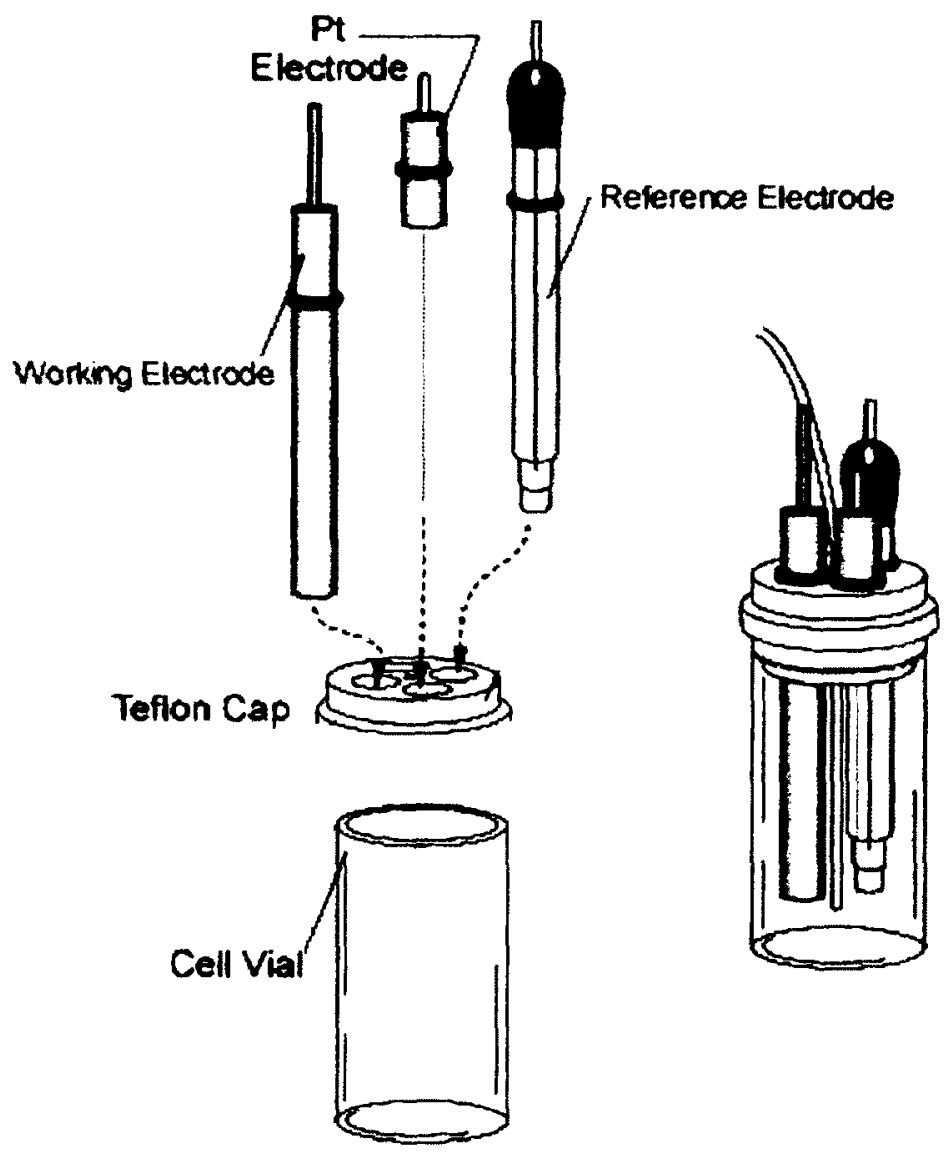

Figure 4.2: 3-electrode cell showing the reference, working, and auxiliary electrodes placed through a Teflon cap and into a fixed volume vial ${ }^{165}$.

\subsubsection{Cyclic Voltammetry}

Cyclic Voltammetry (CV) is one of the most versatile techniques in electrochemistry, where a time-varying potential is applied to the working electrode. Its use has been employed in the past to diagnose mechanisms of electrochemical reactions, for the species present at the electrode and for semi-quantitative rate analysis ${ }^{166}$. It is the technique of choice for non-electrochemists due to its ease of use and manipulation. 
The generated voltammogram is similar to a more common analytical spectrum in that it gives information as a function of change in energy. In CV, the potential of a stationary working electrode is varied in a cyclic manner (see Figure 4.3) in an unstirred solution and the resulting current measured ${ }^{166}$ relative to a reference electrode.

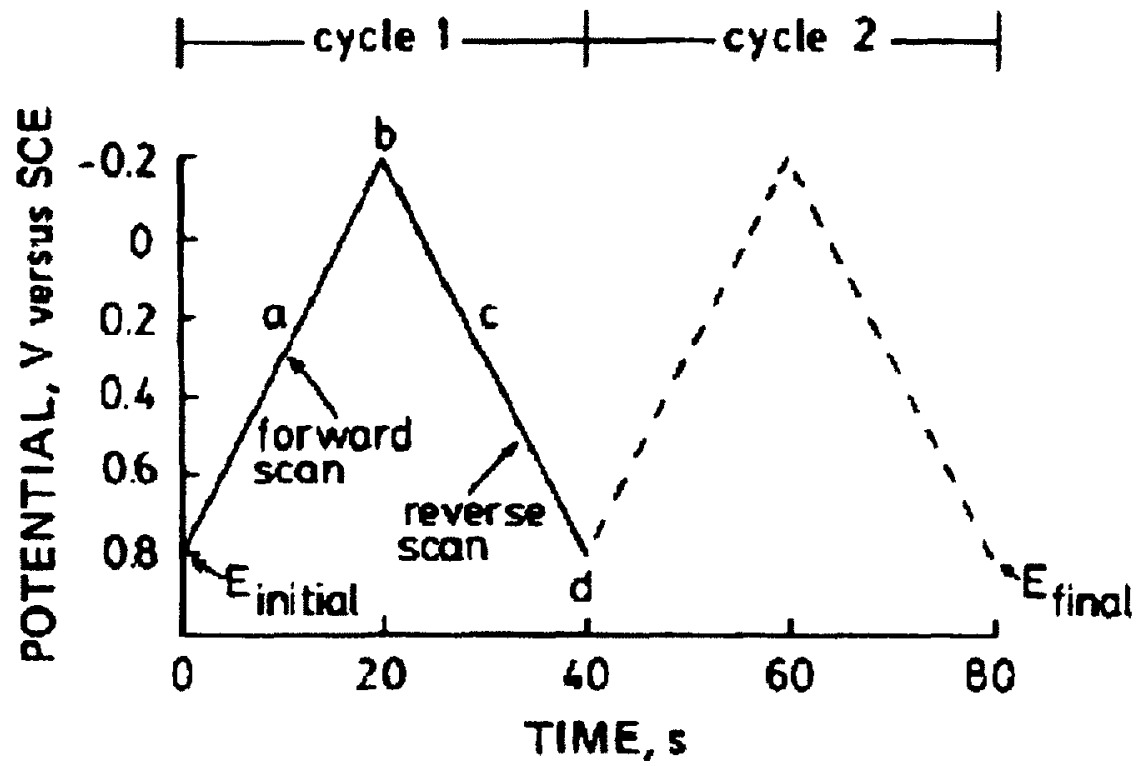

Figure 4.3: Triangular cyclic potentials during a typical cyclic voltammetry scan. The slope of the line is the scan rate. The potential is cycled over a period of time and the changes in current measured ${ }^{166}$.

Considering an example of a typical electroactive probe ferricyanide $(E=0.2 \mathrm{~V}$ vs. SCE; $\mathrm{K}_{3} \mathrm{Fe}(\mathrm{CN})_{6}$ ), one can see its cyclic voltammogram (Figure 4.4 ) as the $\mathrm{Fe}^{2+}$ ions are oxidized to $\mathrm{Fe}^{3+}$ and vice versa for the reduction as it is a reversible system $\left(\mathrm{Fe}^{2+}<->\right.$ $\mathrm{Fe}^{3+}$ ). In the forward direction (top curve), once the potential of the cathodic current is sufficiently negative to reduce $\mathrm{Fe}^{3+}$, the peak starts to develop (point b). From points $\mathrm{c}-\mathrm{d}$ 
the concentration of $\mathrm{Fe}^{3+}$ is rapidly diminished as it is reduced to $\mathrm{Fe}^{2+}$. Points d-f signify depleted $\mathrm{Fe}^{3+}$ as over the time frame of study all the $\mathrm{Fe}^{3+}$ has been depleted to $\mathrm{Fe}^{2+}$. The potential is then cycled backwards in the negative direction and the opposite trend can be seen where the $\mathrm{Fe}^{2+}$ is oxidized to $\mathrm{Fe}^{3+166}$.

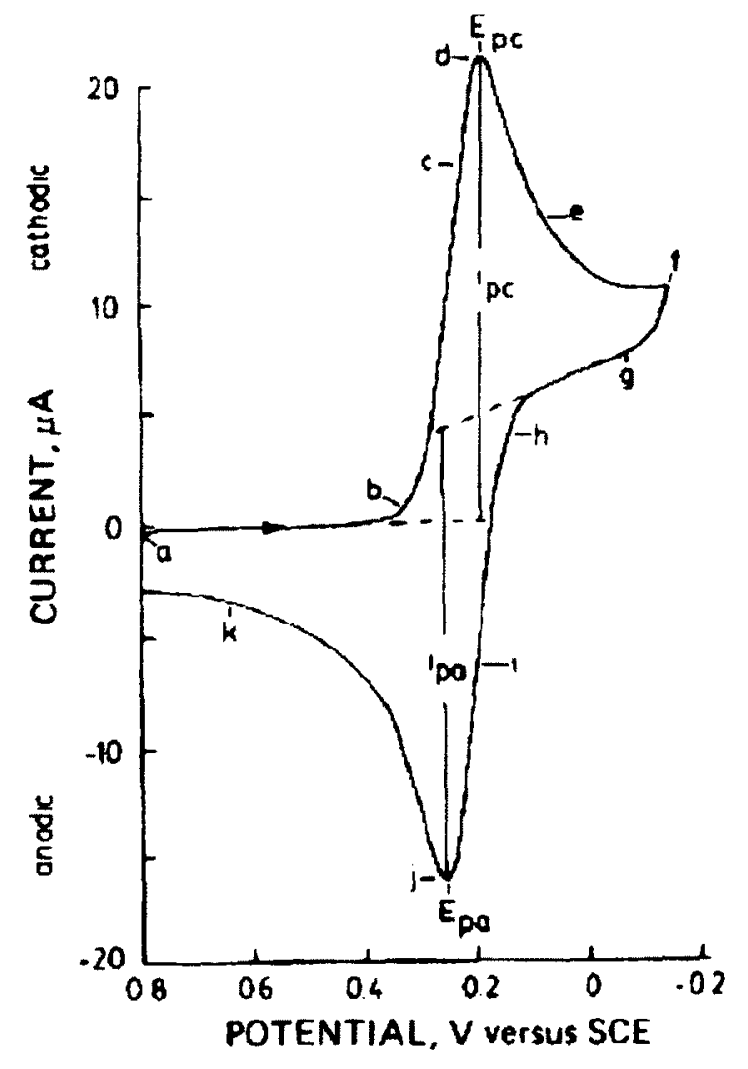

Figure 4.4: Cyclic voltammogram of $\mathrm{K}_{3} \mathrm{Fe}(\mathrm{CN})_{6}$; where $i_{p c}$ and $i_{p a}$ are peak cathodic and anodic currents, respectively; and $E_{p c}$ and $E_{p a}$ are cathodic and anodic peak potentials of a reversible system ${ }^{166}$.

For cyclic voltammetry at planar electrodes in a reversible system (i.e., governed by Faradaic processes) the boundary condition (ratio) of oxidized and reduced species in 
solution can be looked at by the Nernst equation. For simplicity, Equation 6 only considers reaction in one direction (linear sweep). ${ }^{167}$

$\frac{[O]}{[R]}=\exp \left[\frac{n F}{R T}\left(E-E^{\circ}\right)\right]$

where $[O]$ and $[R]$ are concentrations of oxidized and reduced species in solution.

To solve for Equation $6[0] /[R]$, their diffusion equations (equations 7.1 and 7.2 ) need to be solved in the Laplace domain by using their diffusion equations. The Laplace transformation is not shown here but can be found in the cited reference ${ }^{167}$.

$\frac{\partial[O]}{\partial t}=D_{\mathrm{o}} \frac{\partial^{2}[O]}{\partial x^{2}}$
$\frac{\partial[R]}{\partial t}=D_{R} \frac{\partial^{2}[R]}{\partial x^{2}}$

The final result (looking at just oxidized species) after inversion can be expressed as

$$
I=n F A[O]\left(\pi D_{0} \sigma\right)^{1 / 2} \varphi(\sigma t)
$$

Where $\sigma=\frac{n F}{R T} v$ and $\sigma t=\frac{n F}{R T}(E i-E)$

Therefore, the current is dependent on the root of the scan rate. The values for the variable function $\pi^{1 / 2} \phi(\sigma t)$ have to be found numerically ${ }^{167}$ and can be found in the cited reference. The equation further simplifies to: 
$I_{\mathrm{p}}=2.69 \times 10^{5} n^{3 / 2} A D^{1 / 2}[O] v^{1 / 2}$

Where $I_{p}=$ current $(A) ; A=$ area of the electrode $\left(\mathrm{cm}^{2}\right), D=\operatorname{diffusion}\left(\mathrm{cm}^{2} / \mathrm{s}\right) ;[0]=$ concentration of electroactive probe $\left(\mathrm{mol} / \mathrm{cm}^{3}\right)$ and $v=s c a n$ rate $(V / \mathrm{s})$

From equation 9 it is evident that cyclic voltammetry can be used to extrapolate diffusion coefficients in a reversible system driven by faradaic processes (such as mass transport). This equation models 20 linear diffusion through the film, although there are modified forms of this equation to account for non-linear transport ${ }^{168}$. This project utilizes Equation 9 to calculate the mass-transport limited diffusion coefficients of different films bound to the electrode surface. This simplifies the calculations as we are only interested in relative changes in diffusion and not absolute changes in film morphologies (e.g., pore sizes, diffusive path, etc) for which additional extensive characterization would be required.

Studying diffusion of polyelectrolyte films via cyclic voltammetry is a well established technique ${ }^{169,170,171}$. Various groups have studied diffusion coefficients of different films, including organic ${ }^{172}$, metal-based ${ }^{173}$, nanoparticle ${ }^{174}$, and biological ${ }^{175}$ based films. Faradaic processes that result in the reduction or oxidation reactions at the electrode surface can give an indication on the rate of charge accumulation and consequently, the diffusion. This is an attractive approach as it allows for measuring diffusion in real-time with highly sensitive resolution. We have previously shown (Chapter 3 ) that the diffusion of a target molecule can be changed in bilayer films upon aptamer-binding 
using FRAP. We wanted to explore in this chapter if (a) the films behave selectively to positively and negatively charged small molecules (electroactive probes); and (b) if the mode of transport, that is, active or passive transport, can be elucidated from the data. Similar to the previous chapters, in this project we made films using the sulforhodamine B aptamer (SA), a random DNA strand (RO) with the same length as the aptamer, and a film with no DNA present (PSS). We tested the films in the absence of the target (sulforhodamine B) and negative control (tetramethylrosamine) (control), and in the presence of just target (SB) and just negative control (TMR). We wanted to measure the differences of diffusion between the different films and different molecular ions. Any findings would shed light on the transport mechanism and allow for the better development of controlled release systems for our smart fertilizers, in terms of designing the smart fertilizers to facilitate transport through active or passive processes.

\subsection{Experimental}

\subsubsection{Materials}

Synthetic polyelectrolytes and oligonucleotides (DNA) were purchased or synthesized as previously described. Potassium ferricyanide $\left(\mathrm{K}_{3} \mathrm{Fe}(\mathrm{CN})_{6} ; 99 \%\right)$ and hexaammineruthenium chloride $\left(\mathrm{Ru}\left(\mathrm{NH}_{3}\right)_{6} \mathrm{Cl}_{3} ; 98 \%\right)$ were purchased from SigmaAldrich and used as received. 2-Mercaptoethylamine hydrochloride (98+\%) was purchased from Alfa Aesar and used as received. All other reagents were of technical grade and used without further purification. All reactions were at room temperature 
unless otherwise stated. All glassware was rinsed $5 \mathrm{X}$ with distilled and $5 \mathrm{X}$ with deionized water).

\subsubsection{Methods}

Lbl deposition on electrodes: Gold electrodes $(1.6 \mathrm{~mm})$ were polished on a fine polishing pad using $1 \mu \mathrm{m}$ diamond slurry polish. The electrode was first rinsed with water and polished on the pad by moving the electrode in a figure 8 motion for $2-3$ minutes. Once polished, the electrode was further cleaned by etching the working electrode in $1 \mathrm{M}$ $\mathrm{H}_{2} \mathrm{SO}_{4}$ in a Ag/AgCl|Pt 3-cell system from -0.25-1.65V using a CHinstruments $660 \mathrm{C}$ instrument. This step was repeated twice to ensure maximum exposed crystalline surface of the working electrode. This etch was also used to calculate surface area of the planar electrode. We noticed a lot of debris on the electrode surface (i.e., multiple peaks after the $\mathrm{H}_{2} \mathrm{SO}_{4}$ etch) and found that doing multiple polishing steps and using a fresh solution of $1 \mathrm{M} \mathrm{H}_{2} \mathrm{SO}_{4}$ every time cleaned the electrodes appropriately.

To the gold electrode, $100 \mu \mathrm{L}$ of $5 \mathrm{mM} 2-$ Mercaptoethylamine hydrochloride $(98+\%)$ in anhydrous ethanol was added to functionalize the surface with a positively-charged species via a gold-thiol bond. The solution was refreshed every five minutes for a total incubation time of 50 minutes. The electrode was then rinsed with fresh ethanol, and $2 \mathrm{mg} / \mathrm{mL}$ in $0.5 \mathrm{M} \mathrm{NaCl}$ polyelectrolytes (PAH or PSS), and oligonucleotides (ca. $1 \mu \mathrm{mol}$ RO and $\mathrm{SA}$ in $0.5 \mathrm{M} \mathrm{NaCl}$ ) were deposited in the same alternating way as previously described. The oligonucleotides were heated at $70^{\circ} \mathrm{C}$ to ensure that they deposited in a fairly linear form (denatured) rather than as globules. The films were: $(\mathrm{PAH})_{4}(\mathrm{PSS})_{4}$ and 
$(\mathrm{PAH})_{4}(\text { oligo })_{2}(\mathrm{PSS})_{2}{ }^{\mathrm{b}}$; where the oligo bilayers were sandwiched between the synthetic polyelectrolytes. The oligos were the sulforhodamine B aptamer (SA) and random strand (RO) which was the same length as SA (see chapter 2 for the sequences). Layer growth was monitored (see Figure 4.5 ) by the decrease in ferricyanide peak current as a result of increasing bilayer number.

Cyclic voltammetry: $\mathrm{CV}$ was performed on prepared working electrodes using a 3-cell system as discussed above. Potassium chloride (0.5M) was used as the electrolyte, $\mathrm{K}_{3} \mathrm{Fe}(\mathrm{CN})_{6}(0.5 \mathrm{mM})$ as the negatively charged probe; $\mathrm{Ru}\left(\mathrm{NH}_{3}\right)_{6} \mathrm{Cl}_{3}(0.5 \mathrm{mM})$ as the positively charged probe; sulforhodamine B dye (target; $100 \mu \mathrm{M})$ as the target; and tetramethylrosamine (control; $100 \mu \mathrm{M}$ ) as the negative control. Prior to experimentation, it was ensured that the target and control do not interfere with the redox potentials of the probes. The solutions were purged for five minutes with argon before measurements were started. We also found remnants of the electroactive probes remaining in the film when switching the probe causing electrocatalysis (formation of a second reduction peak). This was removed by collecting 50 cycles of the voltammograms and washing thoroughly to remove remaining electroactive probe. $10 \mathrm{~mL}$ of solution was used every time to remove any differences in flux from differing volumes being introduced. A faraday cage was used to isolate the system from electrical and electromagnetic influences. The data was analyzed by extracting the peak current $\left(I_{p}\right)$ using semi-derivative analysis ${ }^{176}$ for better resolution. Surface area was calculated using a $1 \mathrm{M} \mathrm{H}_{2} \mathrm{SO}_{4}$ etch at a sweep rate of $200 \mathrm{mV} / \mathrm{s}$ for a total of 20 segments (Area =

\footnotetext{
${ }^{b}$ The build-up of the oligo films was: PAH/PSS $\times 1$, PAH/oligo $\times 2$, PAH/PSS $\times 1$
} 
peak current $(\mathrm{C}) / \mathrm{charge}\left(\mathrm{C} / \mathrm{cm}^{2}\right)$; where charge $=50 \mu \mathrm{C} / \mathrm{cm}^{2}$ at $\left.200 \mathrm{mV} / \mathrm{s}\right)$. Equation 9 was used to calculate the diffusion coefficients using the following values: $n=1$ (due to loss or gain of one electrode per electroactive molecule); surface area of electrode calculated from equation above from $1 \mathrm{M} \mathrm{H}_{2} \mathrm{SO}_{4}$ etch $\left(\mathrm{cm}^{2}\right) ; I_{p}$ extracted from $\mathrm{CV}$ curves $\left(-0.15\right.$ to $-0.35 \mathrm{~V}$ for $\mathrm{Ru}\left(\mathrm{NH}_{3}\right)_{6} \mathrm{Cl}_{3}$ and 0.3 to $\mathrm{OV}$ for $\left.\mathrm{K}_{3} \mathrm{Fe}(\mathrm{CN})_{6}\right)$ after semi-logarithmic analysis $(A)$; and concentration of electroactive probes was as described in experimental $\left(1 \mathrm{~mol} / \mathrm{L}=0.001 \mathrm{~mol} / \mathrm{m}^{3}\right)$. Statistics were done via a standard $T$ Test using an average of 3 calculated diffusions, 2 tails and two-sample equal variance (homoscendastic) in Microscoft Excel 2007.

\subsection{Results and Discussion}

Various groups have studied the stability, diffusion, and kinetics of polyelectrolyte films using small molecule probes ${ }^{177}$. In this chapter, we wanted to study (a) the diffusion kinetics of small molecules (other than target) upon aptamer-target binding; and (b) if these functional films have charge selectivity which could affect diffusion. We chose small molecular probes since we believe that the trigger for our system, that is, an exudate from the crop of interest, will most likely be a small molecule. Bruening and coworkers (2000) have shown that changing the anionic polyelectrolyte can have a drastic effect on the diffusion of the bilayer films ${ }^{178}$. In their study, they changed the type of negative polyelectrolyte film and found a direct correlation of decreasing diffusion with increasingly negative charge density. They suggest electrostatic repulsion playing a role 
in diffusion, where the negative charge on the termination layer of the film repels incoming charges similar to the function of a electric double layer discussed previously.

\subsubsection{Optimizing number of bilayers}

To explore the changes in diffusion between the various films, the background diffusion, that is the diffusion as a result of film thickness, needs to be minimized to the point that differences in peak current could be quantified in a reproducible manner. This can be thought of as decreasing the diffusion to the level that current to the electrode is lowered and changes in the current can easily be measured. Since polyelectrolyte films are inherently extremely permeable and can act as hosts for many different types of ions as ion sinks, the peak current needs to be controlled (i.e., decreased) by controlling the number of bilayers in the film (increasing number of bilayers should lead to decreased permeability). This has been shown for synthetic polyelectrolyte films ${ }^{179}$. Figure 4.5(a) below shows the $C V$ of a bare gold electrode prior to functionalization after polishing and $1 \mathrm{M} \mathrm{H}_{2} \mathrm{SO}_{4}$ etch; and (b) shows the effect of number of bilayers on the diffusion of ferricyanide on the gold working electrode. 4 bilayers ( 8 layers) were chosen for our diffusion experiments because it gave us a low peak current with a relatively thin film which would allow the possible detection of small increases in the peak current due to possible aptamer-target binding. 

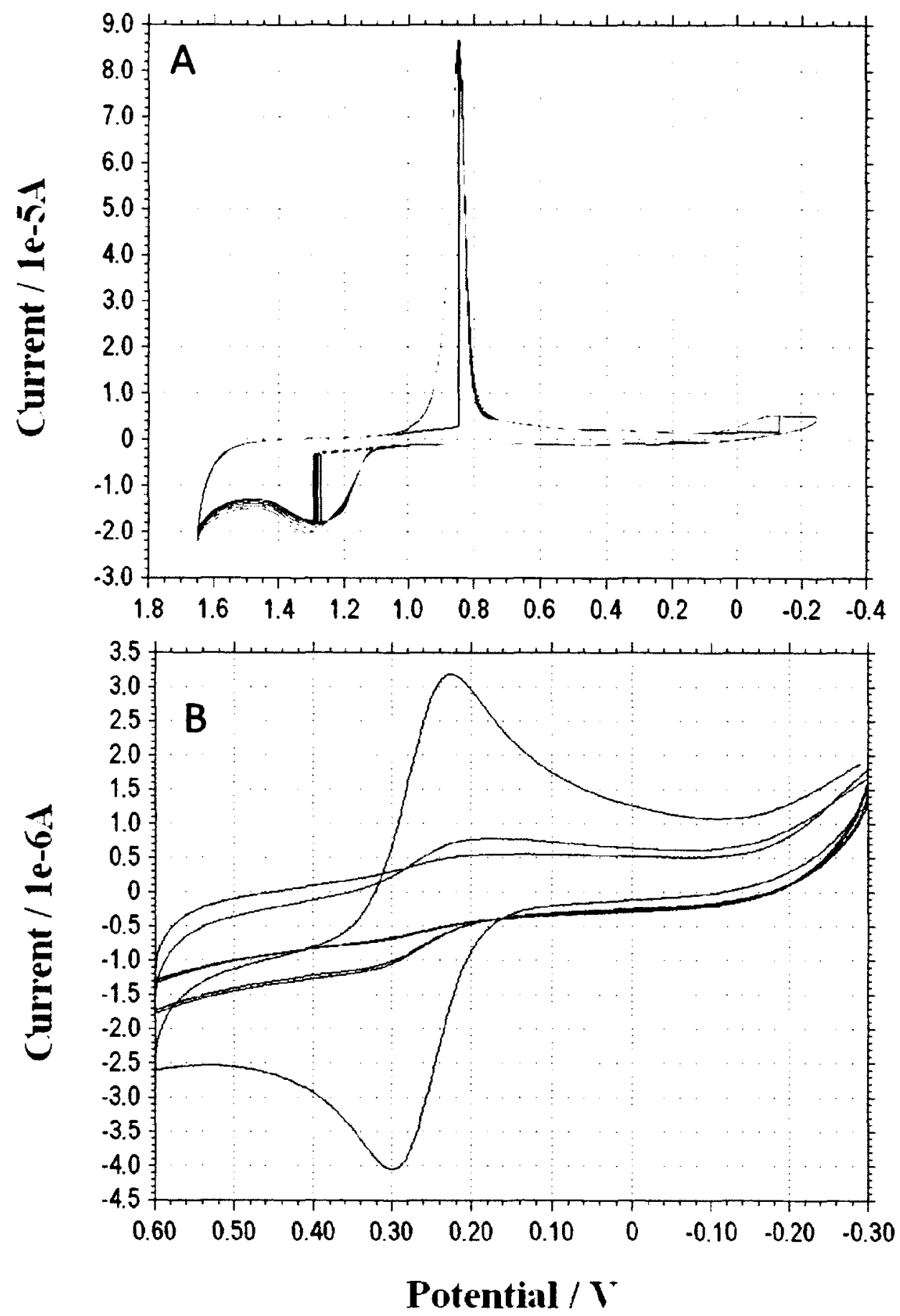

Figure 4.5: (a) $\mathrm{CV}$ of bare gold electrode after polishing and $1 \mathrm{M} \mathrm{H}_{2} \mathrm{SO}_{4}$ etch; and (b) $\mathrm{CV}$ of different number of bilayers (PAH/PSS) with $0.5 \mathrm{mM}$ ferricyanide: purple ( 2 bilayers); green ( 4 bilayers); and blue ( 6 bilayers) showing decreasing current on the electrode as a result of increasing number of layers. 


\subsubsection{Cyclic Voltammetry}

As has been discussed in Chapter 2, there is an effect of annealing on the diffusion of functional polyelectrolyte films, which allows for denaturing of the aptamer and better refolding around its target. To test this, we studied the changes in diffusion, both before and after annealing at $70^{\circ} \mathrm{C}$ for 10 minutes. To do this we took all the measurements for the two probes prior to annealing, then annealed each electrode prior to remeasuring in the electrolyte solutions. A typical CV curve of films tested, before and after annealing, with $(\mathrm{A}) \mathrm{K}_{3} \mathrm{Fe}(\mathrm{CN})_{6}$ and $(\mathrm{B}) \mathrm{Ru}\left(\mathrm{NH}_{3}\right)_{6} \mathrm{Cl}_{3}$ are shown below in Figure 4.6 along with the semi-derivative analysis (inset). 

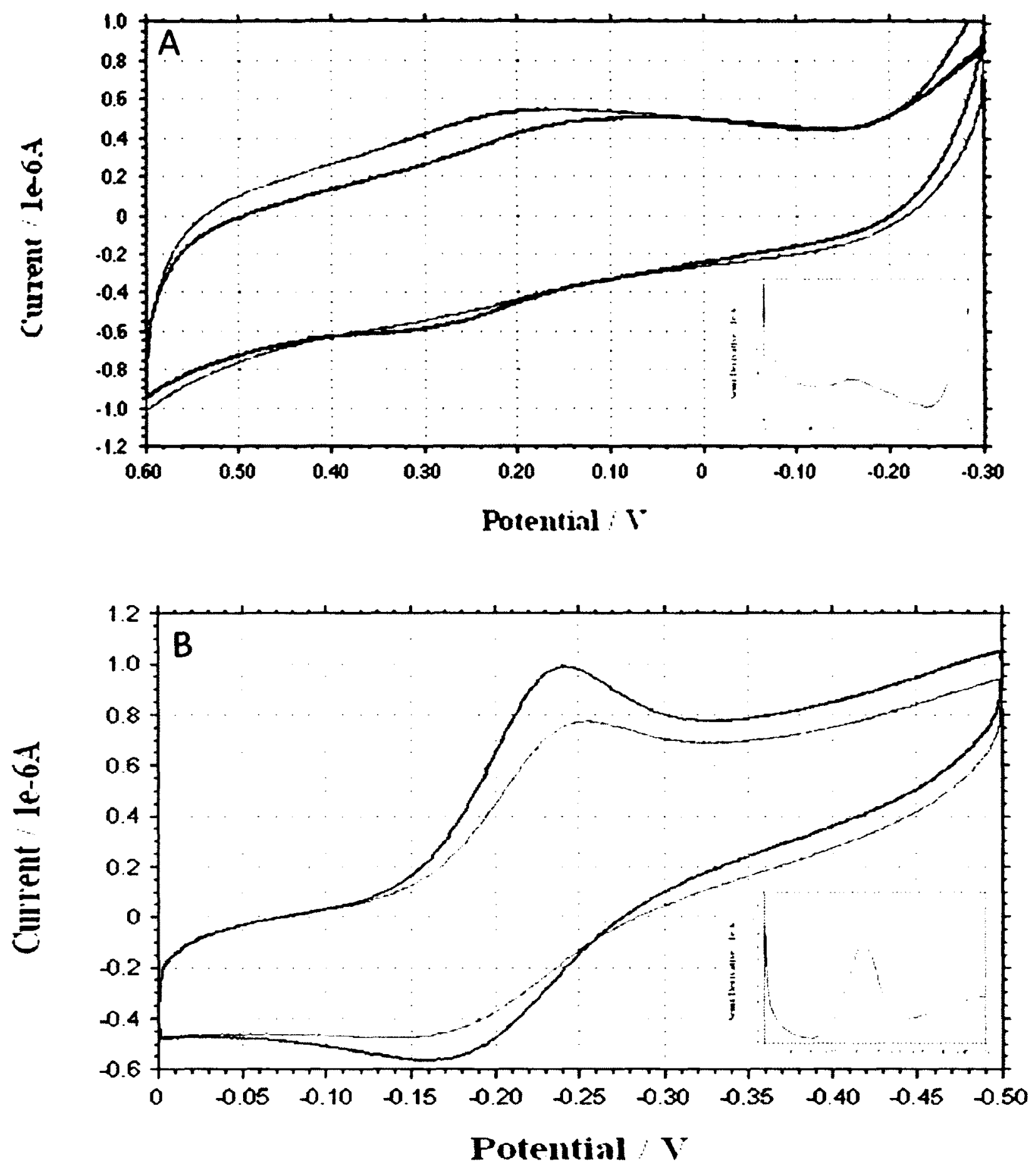

Figure 4.6: $\mathrm{CV}$ of bilayer films with (A) $\mathrm{K}_{3} \mathrm{Fe}(\mathrm{CN})_{6}$ and (B) $\mathrm{Ru}\left(\mathrm{NH}_{3}\right)_{6} \mathrm{Cl}_{3}$ before (blue) and after (red) annealing with semi-derivative analysis (inset). 
There is a slight shift in the peak current, likely due to the effect of annealing on the film morphologies $^{180}$. As has already been shown ${ }^{181}$, semi-derivative analysis can increase resolution and provide an accurate quantification of peak current. The semi-derivative transformation allowed for much better resolution and reproducibility.

Voltammograms were collected for three different films: (1) synthetic film with no DNA $(\mathrm{PAH})_{4}(\mathrm{PSS})_{4}[\mathrm{PSS}]_{;}(2)$ synthetic film with embedded random strand $(\mathrm{PAH})_{4}(\mathrm{RO})_{2}(\mathrm{PSS})_{2}$ [RO]; and synthetic film with embedded aptamer $(\mathrm{PAH})_{4}(\mathrm{SA})_{2}(\mathrm{PSS})_{2}$ [SA]. The films were tested (1) in the absence of target and control (i.e., just electrolyte and probe) [Contro] to which the data was normalized; (2) with a negative control (tetramethylrosamine) [TMR]; and (3) in the presence of target (sulforhodamine B) [SB]. The diffusions were calculated using Equation 9.

For the following studies, the conditions were as follows: sweep rate $=0.1 \mathrm{v} / \mathrm{s}$; concentration of $\mathrm{K}_{3} \mathrm{Fe}(\mathrm{CN})_{6}$ and $\mathrm{Ru}\left(\mathrm{NH}_{3}\right)_{6} \mathrm{Cl}_{3}=5 \mathrm{~mol} / \mathrm{m}^{3}$. Three different electrodes were used to generate the data and the data corrected for surface area (through equation 1) and normalized against control .

Data for before and after annealing for $\mathrm{K}_{3} \mathrm{Fe}(\mathrm{CN})_{6}$, the negative probe, are presented below in Figure 4.7. 

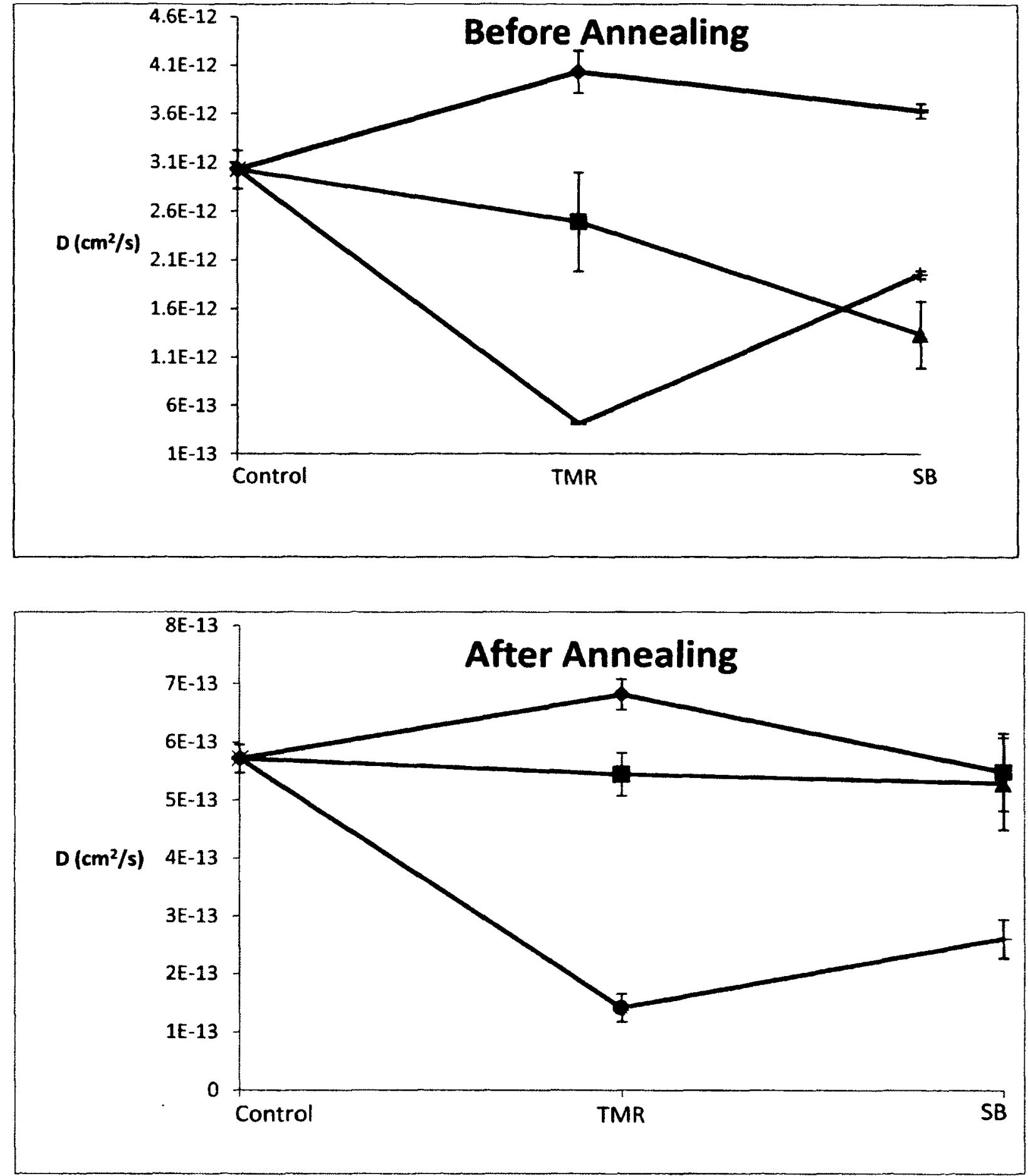

Figure 4.7: Normalized Diffusion coefficients $\left(\mathrm{cm}^{2} / \mathrm{s}\right)$, before and after annealing, with $\mathrm{K}_{3} \mathrm{Fe}(\mathrm{CN})_{6}$ of aptamer film (SA; blue); random DNA film (RO; green); and synthetic films with no DNA (PSS; red) in the absence of target or control dye (control); presence of control dye (TMR); and target $(S B)[$ Red = PSS; Green = RA; Blue = SA]. 
The calculated diffusion coefficients for ferricyanide are on the order of $10^{-16}$ to $10^{-}$ ${ }^{17} \mathrm{~m}^{2} / \mathrm{s}$, with annealing causing a ca. one order of magnitude decrease in diffusion which is consistent with literature showing decreased diffusion upon heating of polyelectrolyte films $^{182}$. The calculated diffusion coefficients are low. Indeed, it has been shown that when a negative termination layer is used as is the case in our situation (PSS), electrostatic repulsion counters diffusion of ferricyanide (and other negatively charged molecules) through the polymer film ${ }^{183}$. Thus, our data for the negative probe correlates with findings in literature showing very low diffusion. Nonetheless, some trends can be noted for our negatively terminated polyelectrolyte film in contact with negatively charged molecules. Using $\mathrm{K}_{3} \mathrm{Fe}(\mathrm{CN})_{6}$ [probe], in films PSS and RO, the diffusion of the probe in points $T M R$ and $S B$ is reduced relative to Control (i.e., the first point). This is predominantly a consequence of decreased mass flux of the probe through the films. In the presence of the target (SB) and negative control (TMR), additional molecules compete to permeate the films and the overall flux is decreased. By this argument, the diffusions of all films in Control, thus, should be the highest which is why the relative flux of TMR and SB are lower in all cases. The diffusion with film SA is different than the other films in the case of before annealing, in that there is little decrease in relative diffusion as is the trend with the other two films. However, the diffusion does not exceed that of control (i.e., it does not become more permeable), and accounting for the standard deviation being lower than control, suggesting no statistically significant trend can be extrapolated between the films or amongst points control, TMR, and SB. Moreover, as can be seen with the data after annealing, there is no statistical difference 
( $p>0.05$ ) between the films PSS and SA, thereby, showing the better resolution of the data after annealing. The data also shows statistical insignificance between film SA and points Control, TMR, and SB. Thus, it is not possible to extrapolate the change in diffusion after aptamer-target binding using a negative probe $\mathrm{K}_{3} \mathrm{Fe}(\mathrm{CN})_{6}$. We believe that the lack of discernible changes is primarily due to two reasons. First, the mass flux ${ }^{184}$ is decreased upon addition of the target and control dye, thereby, decreasing the overall diffusion coefficients. Second, as has already been discussed ${ }^{185}$, negatively charged films will have significant effects on the diffusion of negatively charged probes due to electrostatic repulsion. It is then possible that the effect of charge masks any true diffusion (if any) from aptamer-target binding and as such cannot be measured under these test conditions. Moreover, it is apparent that the data for after annealing gave much better resolution in terms of diffusions. To find effects on diffusion from aptamertarget binding and remove confounding changes on the diffusion from electrostatic repulsion, we studied next, a positively charged probe $\mathrm{Ru}\left(\mathrm{NH}_{3}\right)_{6} \mathrm{Cl}_{3}$ [probe]. The diffusions using the positive probe are shown below in Figure 4.8. 

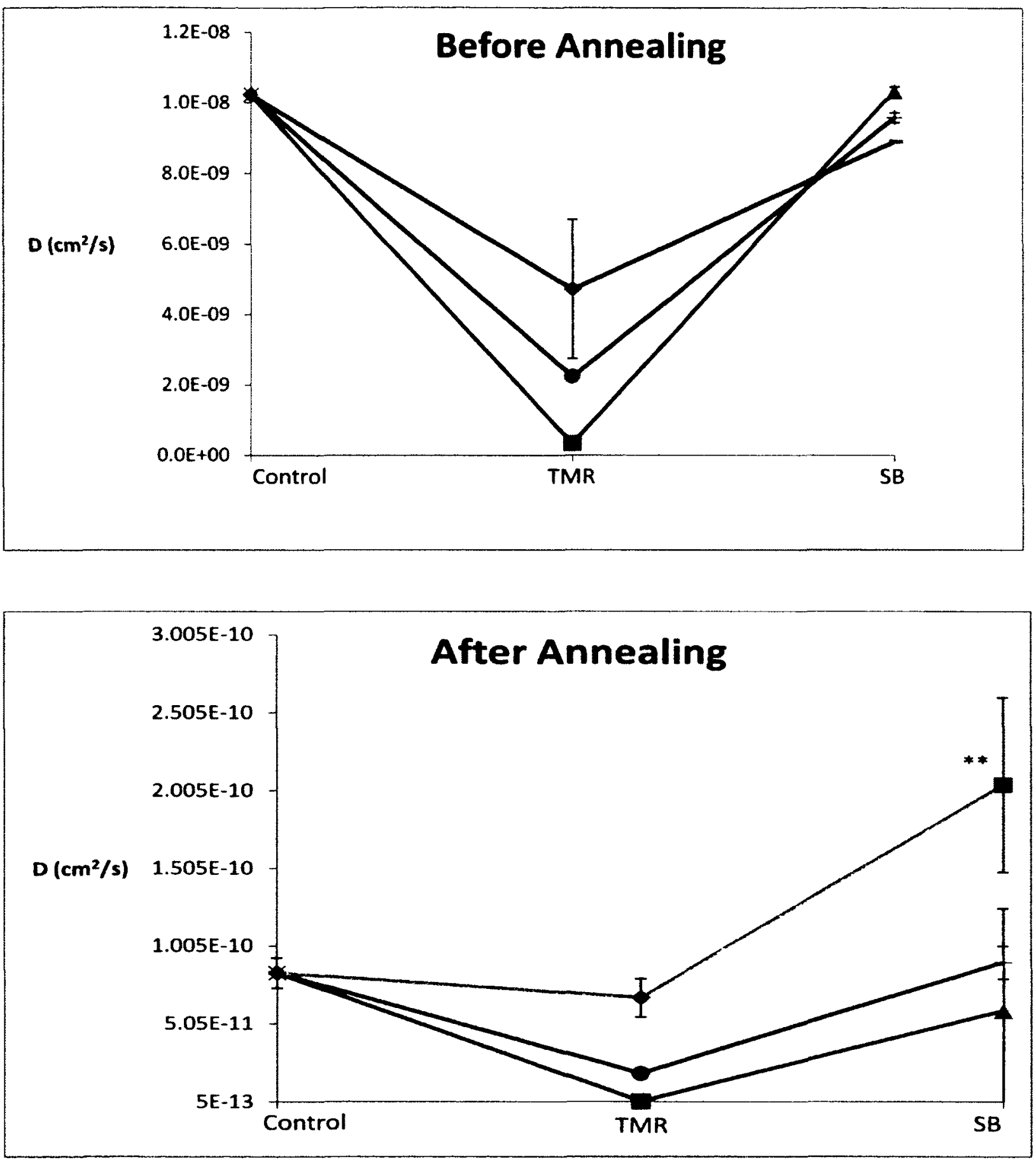

Figure 4.8: Normalized Diffusion coefficients $\left(\mathrm{cm}^{2} / \mathrm{s}\right)$, before and after annealing, with $\mathrm{Ru}\left(\mathrm{NH}_{3}\right)_{6} \mathrm{Cl}_{3}$ of aptamer film (SA; blue); random DNA film (RO; green); and synthetic films with no DNA (no DNA; red) in the absence of target or control dye (control); presence of control dye (TMR); and target $(S B) .{ }^{* *}$ indicates significant difference $(p<0.05)$ between the three films and TMR and SB of the SA film. [Red = PSS; Green = RA; Blue = SA] 
The diffusion data for the positive probe had higher reproducibility. The calculated values were on the order of $10^{-14}-10^{-16} \mathrm{~m}^{2} / \mathrm{s}$. These diffusion coefficients are consistent with our findings for the target dye in Chapter 3 and with the diffusion values in literature of small molecules through PAH/PSS films ${ }^{186}$. These films do not suffer the effects of electrostatic repulsion and therefore changes in film diffusion are more evident. Before annealing, we saw similar trends for all three films with no significant changes in diffusion ( $p>0.05)$. After annealing data shows a significant increase $(p=$ $0.009-0.02 ;<0.05$ ) in diffusion of the positive probe upon aptamer-target binding (SA film), compared with films PSS and RO and control and TMR. The decrease in diffusion upon addition of TMR is likely due to the decrease in flux as TMR and the electroactive probe compete to diffuse through the film and onto the electrode. The significant increase in diffusion of the SA film with target suggests that the loss of flux of electroactive probe is compensated and the overall increase is higher than the electroactive probe alone. Thus, we were able to show a significant increase in diffusion of the aptamer film upon binding to its target. As has been previously discussed, the annealing allows for a higher number of unfolded DNA strands in the film which are able to subsequently fold better in the aptamer film upon target introduction, thereby, increasing the signal and resolution.

These findings suggest that the diffusion of molecules through the film is driven predominantly by passive rather than active processes. We have already shown (Chapter 3) the increased diffusion of target through microcapsules upon aptamer- 
target binding as studied by FRAP. The FRAP data could suggest an active transport of target through the film as aptamer binds to the target and subsequently directs it through the polymer film as it binds to a free target molecule from solution (i.e., in a dynamic process). However, the data presented here supports a more passive behavior that is, when the aptamer binds to the target, there is an increase in film deformation and pore formation which increase the mass flux through the film, thereby increasing diffusion for both the target and positive probe. Therefore, towards our goal of understanding the mechanism of release through polymer bilayer films, it is apparent that aptamers have the ability to influence passive diffusion of small molecules, which is an attractive feature for controlled delivery. Although not explored further in this project, it is possible to elucidate changes in diffusion for negatively charged small molecules using a different model system, that is, using films with low permeability and a positive charge on the terminating layer. In our case, we were limited to using negatively charged films as terminating films with the positive polyelectrolyte (PAH) would cause ionic attraction of the target (SB) and control (TMR) to the film. Moreover, since the changes in diffusion with a negative polyelectrolyte could be small, such a system could be tested on microelectrodes to decrease the surface area and thus increase sensitivity. 


\subsection{Conclusions}

From our experiments we saw an increase in the permeability of our films to small molecules upon aptamer-target binding. This suggests that there may be an increase in the size and/or number of pores in the film. We found that, in the case of our positive probe, the diffusion of small molecules was increased as a consequence of aptamertarget binding. This is promising in the development of our smart fertilizer since it allows for understanding the mechanisms of aptamer-target binding and thereby allows for the better design of our final system for in situ testing.

\subsection{Acknowledgements}

Y.S would like to thank Prof. Lai for allowing us to borrow and use his Faraday cage. 


\section{Chapter 5. A model soil molecular target: Lysine}

\section{$\underline{5.1 \text { Introduction }}$}

We have demonstrated that aptamers retain their binding ability (Chapter 2) and that diffusion of target (Chapter 3) and small molecules (Chapter 4) can be altered upon aptamer-target binding. We next want to apply our model thin film-biosensor system to a model plant exudate L-lysine. There is no aptamer in literature for L-lysine, however, an aptamer for ethanolamine has been shown to bind to L-lysine as well. In this project, we first determined the $K_{d}$ of this aptamer so we could optimize our FRAP experiments to calculate diffusion coefficients for microcapsules containing this much larger aptamer strand. $K_{d}$ determination was assessed using FT-IR and fluorescence anisotropy and the FRAP was performed on the Confocal laser scanning microscope using the methods developed in Chapter 3.

\subsubsection{Fluorescence}

Fluorescence is the spontaneous light emitted as electrons return from excited singlet states $\left(S_{n}\right)$ to the ground state $\left(S_{0}\right)^{187}$. The electronic states that exist in a molecule and the transitions between those states can be visualized by Jablonski diagrams (Figure 5.1). As figure 5.1 shows, electrons when excited by a quantum of radiation (photons) will be excited from their ground single state $\left(S_{0}\right)$ to higher excited singlet states of $S_{1}$ and $\mathrm{S}_{\mathrm{n}}{ }^{188}$. On the right side of the diagram, a transition between a ground triplet state $\left(T_{0}\right)$ and its excited states $T_{2}$ can be seen. In such transitions, along with being excited, 
the electrons also undergo a reversal of spin during excitation. A fluorophore is a molecule which has the ability to absorb photons (light) and excite electrons to an excited state leading to fluorescence.

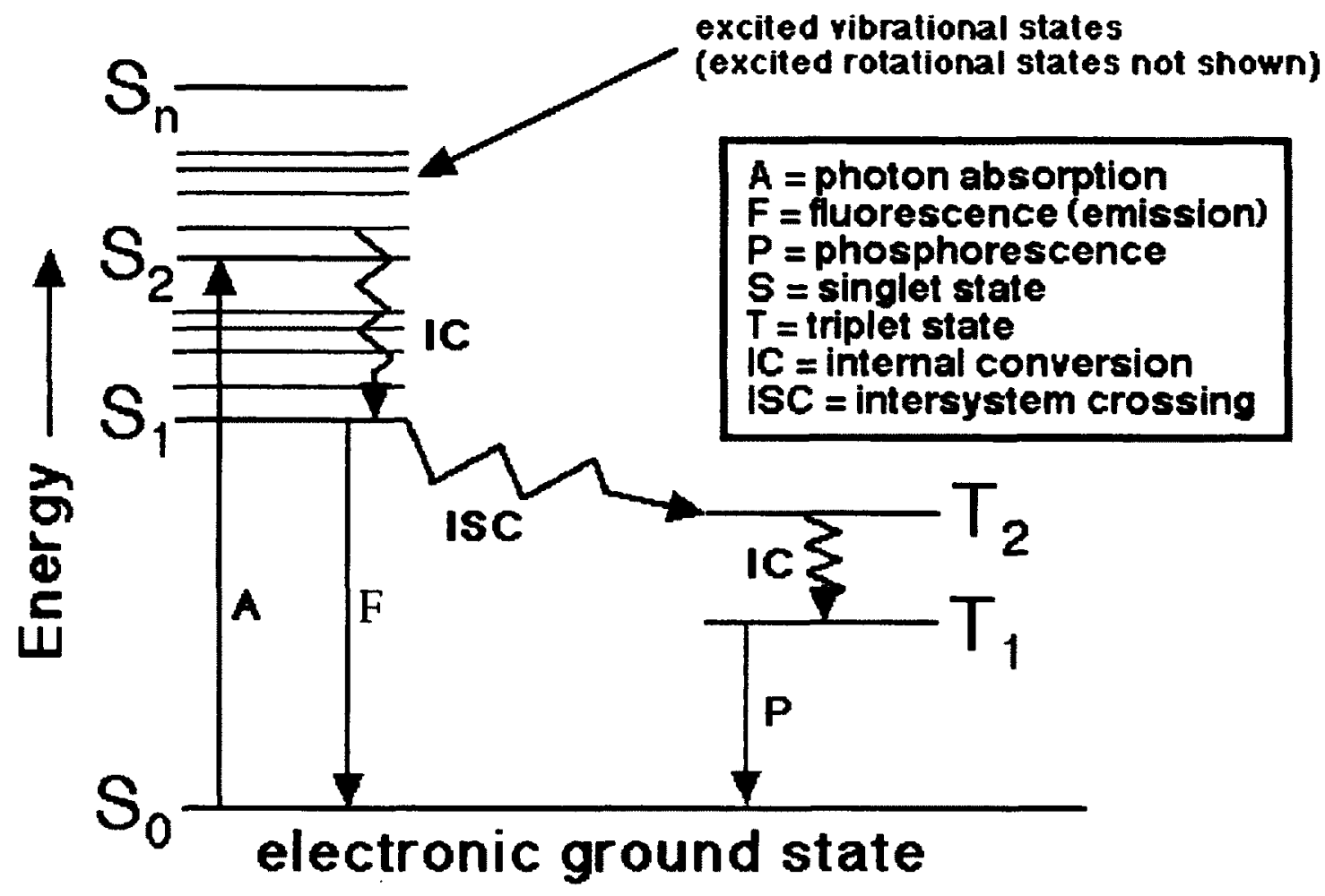

Figure 5.1: Jablonski diagram showing transitions from $S_{0} \rightarrow S_{n}$ and $S_{n} \rightarrow S_{0}$ on the left and the transitions $T_{n} \rightarrow T_{0}$ on the right.

Fluorescence emission is almost always seen exclusively in molecules which contain conjugated systems of bonds, i.e., systems containing single and double bonds, or $\pi$ systems. This is because molecules which lack these characteristics will absorb light and deactivate from the excited state by intermolecular collisions before fluorescence can be achieved ${ }^{189}$. Conjugated systems, however, can stabilize the excited molecule long 
enough (on the order of femtoseconds) that fluorescence transitions can be measured $^{189}$. Time spent in the excited state is characteristic of the molecular environment and fluorescence emission wavelength. Another consideration in fluorescence is the fluorophores quantum yield, that is its efficiency. Quantum yield is defined as the ratio of quanta emitted to the number absorbed, which ranges significantly amongst fluorophores. In extreme cases, irradiation with very high energy photons can result in photodegradation of the fluorophore ${ }^{189}$.

During spectrum measurement, a solution is placed in a cuvette and placed into the fluorescence instrumentation. The sample is irradiated with light at the fluorophores characteristic wavelength, i.e., to promote $S_{0}->S_{n}$ transitions and the emission intensity is measured at a characteristic wavelength. Figure 5.2 shows a typical spectrum, where the absorbance, i.e., $S_{0} \rightarrow S_{n}$ transitions where no light is being emitted and there is only excitation (valleys) and emission, i.e., $S_{n} \rightarrow S_{0}$ transitions of light emission (peaks) are shown. The model spectrum at the bottom shows the difference between absorption and emission spectra (i.e., Stokes shift). 


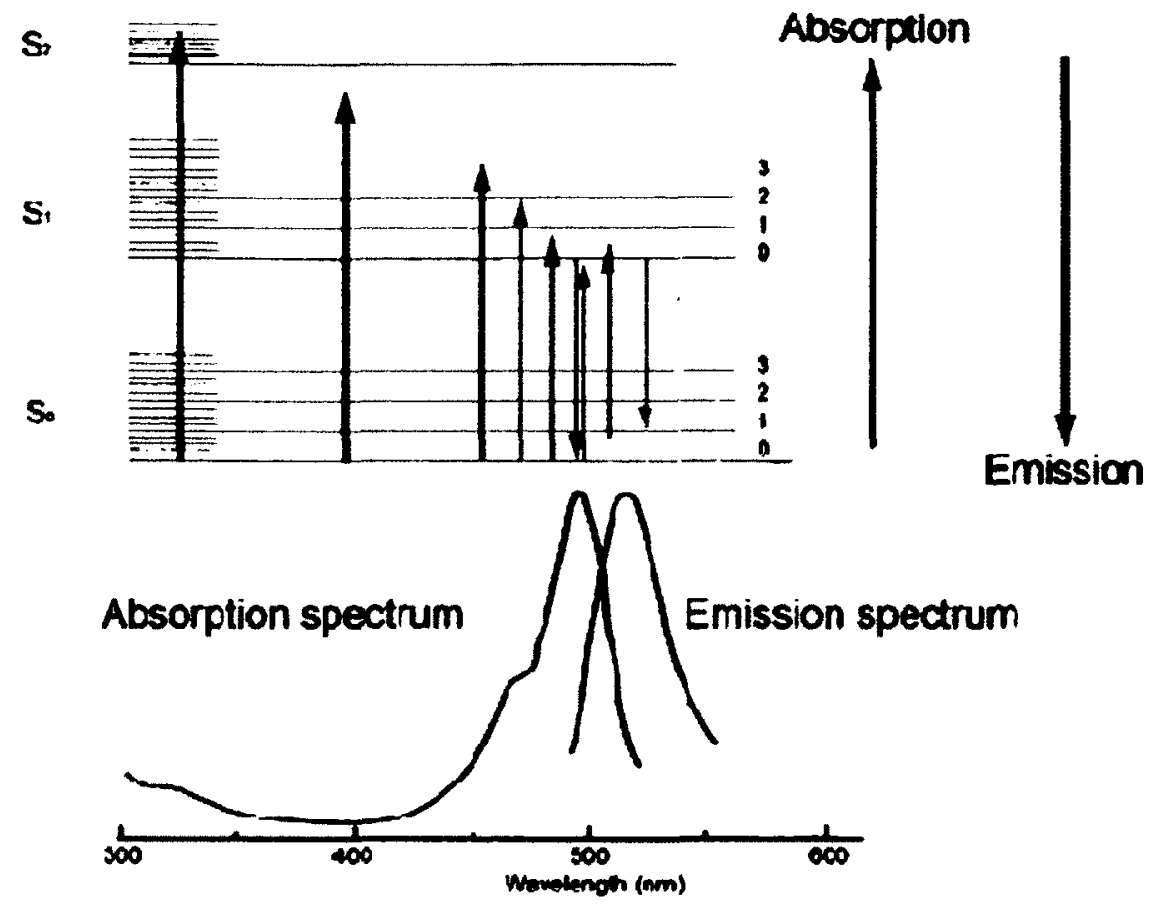

Figure 5.2: Fluorescence spectrum (bottom) showing absorbance transitions $\left(S_{0} \rightarrow S_{n}\right)$ which is represented by valleys and the emission transitions $\left(S_{n} \rightarrow S_{0}\right)$ which are represented by peaks. The overlap between the absorption and emission spectrum is the Stokes shift ${ }^{190}$.

As can be predicted, the chemical environment of the fluorophore can greatly change the resultant emission profile. Solution conditions such as $\mathrm{pH}$, ionic strength, and temperature can greatly change the stability of the excited state ${ }^{191}$, thereby, increasing or reducing the emission peak or shifting the resulting peak (change in Stokes shift). Chemical modification may also have a drastic effect on fluorescence emission. In fact, there are very few examples in literature where a chemical modification has had little effect on the resultant fluorescence profile ${ }^{192}$. Fluorescence may also be quenched under various conditions, where no light is emitted despite effective excitation. 
Manipulating the mechanisms discussed herein, including wavelength, quantum yield, excitation time, chemical environment, and fluorescence has been used to study variety of parameters, including time-resolved or steady-state kinetics, polarization, and anisotropy ${ }^{193}$. For the purpose of this chapter, we will focus on fluorescence (or emission) anisotropy.

\subsubsection{Fluorescence anisotropy}

In solution, molecules rotate freely without any defined orientation. In a fluorescence anisotropic field, fluorophores with an absorption transition dipole aligned with the excitation field are selectively excited and detected. Typically in fluorescence anisotropy, the vertically and horizontally aligned emission field is measured (with polarizers) along horizontal and vertical excitation fields. Modern instruments will measure many different combinations of horizontal and vertical fields. Rotational diffusion, the rotation of the fluorophore within its excited state lifetime, is very fast for small molecules, on the order of nanoseconds. This can impact absolute anisotropy values as the fluorophore does not stay aligned with the excitation field long enough and undergoes random orientations, thereby, decreasing absolute anisotropy. For example, small fluorophores in a non-viscous solution (such as water) will have very low measured anisotropy values due to the inability of the fluorophore to stay aligned long enough to the applied field. If the fluorophore is bound to a large molecule (e.g., protein), the absolute anisotropy values are increased as it 'slows' the rotational 
diffusion of the fluorophore within the excited-state lifetime thereby keeping the molecule aligned for the duration of the applied excitation field. In cases where the bound molecule is relatively small, the change in rotational diffusion may not slow the fluorophore sufficiently to increase anisotropy values, making changes in anisotropy of small molecules hard to measure. For large biological samples such as proteins, the excited-state lifetimes roughly correlate with the rotational diffusion rate of fluorophores and thus slight variations in conditions, including viscosity, temperature, $\mathrm{pH}$, etc can change drastically the measured anisotropy values ${ }^{194}$. To measure anisotropy, the instrument detects the changes in polarization in the horizontal and vertical directions (relative to the propagation of light; Figure 5.3). The ratio of these different absolute anisotropy values forms the basis for fluorescence anisotropy (Equation 2): 


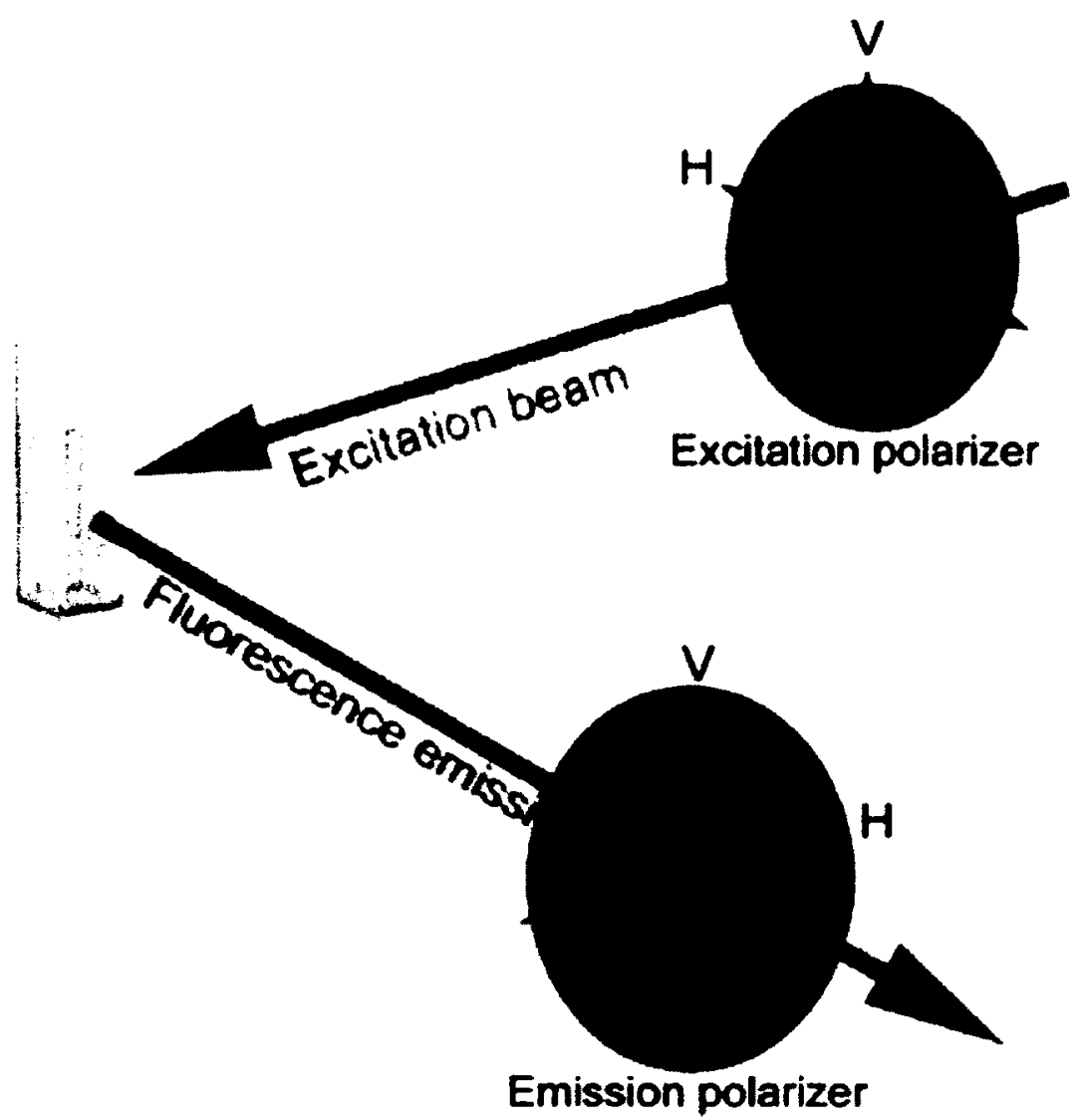

Figure 5.3: Typical setup to study fluorescence anisotropy ${ }^{195}$. This image is taken from the technical notes of the manufacturer of the instrument used in our laboratory.

Consider the following emission intensities: $I_{w}$ where the excitation and emissions polarizers (directions) are aligned vertically with respect to the propagating light, $I_{H H}$ where the excitation and emission polarizers are aligned horizontally, $I_{V H}$ where the excitation polarizer is aligned vertically and emission polarizer aligned horizontally, and vice versa $I_{H V}$ with respect to the propagating light. The anisotropy $(A)$ is then defined as ${ }^{196}$ :

$A=\frac{I_{\mathrm{W}}-G *\left(I_{\mathrm{VH}}\right)}{I_{\mathrm{V}}+2 G * I_{\mathrm{VH}}}$

Where; $G=\frac{I_{\mathrm{HV}}}{I_{\mathrm{HH}}}$ 
Detailed derivation of anisotropy, including static and anisotropy decay can be found in the cited reference ${ }^{197}$. Fluorescence anisotropy is routinely used to calculate binding affinities of biological molecules, including aptamers. A fluorescently tagged aptamer will have a rotational diffusion, and in turn absolute anisotropy, different than when it is bound to its target which slows the rotational diffusion (or in the case of a fluorescent target, the target will slow upon binding to an aptamer). By measuring the difference in anisotropy at different concentrations, one can measure a concentration dependence on the change in anisotropy which is a function of aptamer-target binding. At low concentrations of the target, few molecules are bound to the aptamer, while at higher concentrations many or all target molecules are bound. Using this concentration vs. number of molecules bound (signal) curve and Equations described in Chapter 1, a $K_{d}$ can be determined.

\subsubsection{Fourier-Transform Infrared Spectroscopy}

The Electromagnetic (EM) spectrum is the range of all possible wavelengths of light. In the case of Infrared waves, these frequencies are between $10^{11}-10^{14} \mathrm{~Hz}$ and fall between those of visible and UV light. Infrared Spectroscopy (IR), then, is the study of how infrared light interacts with matter ${ }^{198}$. IR can provide diagnostic data on the types and amount of chemical species present in a solution (provided they absorb in the infrared region). When molecules absorb light (photons), they will vibrate and/or rotate in different spatial directions at their resonance frequencies. The types of vibrations or 
rotations are governed by the complexity of the shape of the molecule. Linear molecules (e.g., diatomic systems) have $3 \mathrm{~N}-5$ degrees of vibrational modes present, while nonlinear (e.g., $\mathrm{H}_{2} \mathrm{O}$ ) will have $3 \mathrm{~N}-6$ vibrational modes, where $\mathrm{N}$ is equal to number of atoms in the molecule. For example, $\mathrm{H}_{2}$, which is a linear molecule, will have one vibrational mode in the infrared region, while water will have 3. Indeed, the IR spectrum of water shows a symmetric stretch, antisymmetric stretch, and a bending vibrational mode. IR spectra are typically measured in units of frequency $\left(\mathrm{Hz}\right.$ or s $\left.\mathrm{s}^{-1}\right)$ due to Fourier transformation (see discussion later), but are typically reported in the units of wavenumbers $\left(\mathrm{cm}^{-1}\right)$. For the remaining of this discussion, wavenumbers and frequency may be used interchangeably, because of their proportionality. While frequency of light can be thought of number of peaks per unit time (s), wavenumbers can be thought of as number of peaks per unit length $(\mathrm{cm})$. Assuming a harmonic oscillator and a simple diatomic molecule, the frequency of vibration $\left(v_{0}\right)$ can be calculated by ${ }^{199}$ :

$$
v_{0}=\frac{1}{2 \pi} \sqrt{\mathrm{k} / \mu}
$$

Equation 3 is the fundamental equation for IR. The dependence of the frequency of motion on the movement of molecules $(k)$, gives a direct measurement of the type of molecules (i.e., functional groups) of the same reduced mass vibrating at a certain energy (wavelength). By invoking the Born-Oppenheimer approximation one can predict the vibrational and rotational states ${ }^{199}$. Turns out that contrary to what we see with fluorescence, the vibrational state can only change to a neighboring state and must be 
accompanied by a rotational change at the same time. That is, rotational and vibrational changes are always \pm 1 .

In reality, diatomic and other complex molecules do not follow a simple harmonic oscillator, corrections using equation 4 (below), a power series, can be made to conform to realistic conditions in solution. These corrections treat the system as an anharmonic oscillator and allow for more realistic measurements. Thus, by knowing the overall energy and vibrational frequency, a spectrum of the actual frequency which is indicative of the molecules present can be determined. Extensions of equation 3 and 4 form the basis for molecules which have more than just one vibrational frequency, that is for molecules with more than 2 atoms. Derivations for more complex systems are not discussed in this chapter, and can be found in the cited reference ${ }^{199}$.

$E_{n}=h v_{0}\left[(v+1 / 2)-x_{e}(v+1 / 2)^{2}+y_{e}(v+1 / 2)^{3} \ldots\right]$

Based on the number of chemical environments present in a sample, and the overlap between resonance frequencies (energies), IR spectra can be a combination of many peaks, that is, low resolution. In such case, Fourier transformations have been used over the past five decades, which breaks the signal (in our case vibrational frequencies) into constituent frequencies, by using the complex Fourier coefficient (equation 5$)^{200}$.

$$
x(t)=\int_{-\infty}^{\infty} S(f) e^{\mathrm{i} 2 \pi \mathrm{ft}} d f \text { and } S(f)=\int_{-\infty}^{\infty} x(t) e^{\mathrm{i} 2 \pi \mathrm{ft}} d t
$$


Where the Fourier coefficient is written as a function of time $X(t)$ or in terms of frequency $S(f)$ of the aperiodic function.

Use of the Fourier transformations to IR (FTIR) has lead to identifications of very subtle frequencies in the IR spectrum and is the reason that IR is being used today with significant analytical power.

In a typical FTIR instrument, the sample is analyzed wet, in a cuvette or between salt plates, or dry as a pellet. The materials used for sample holding must be transparent to the IR region of interest. The optics are set up, conventionally, using a Michelson interferometer (Figure 5.4). A beam splitter splits the light, where $50 \%$ is sent to the sample, and $50 \%$ is reflected to a mirror. The light sent to the sample is focused, and difference between the reflected and sample light is subtracted (by measuring constructive or destructive waves as a change in frequency). This, if done at all wavelengths of the infrared region, will give a spectrum, where the peaks will correspond to absorbed light at a characteristic resonance frequency of the chemical bond. Thus, one can identify the chemical species present. 


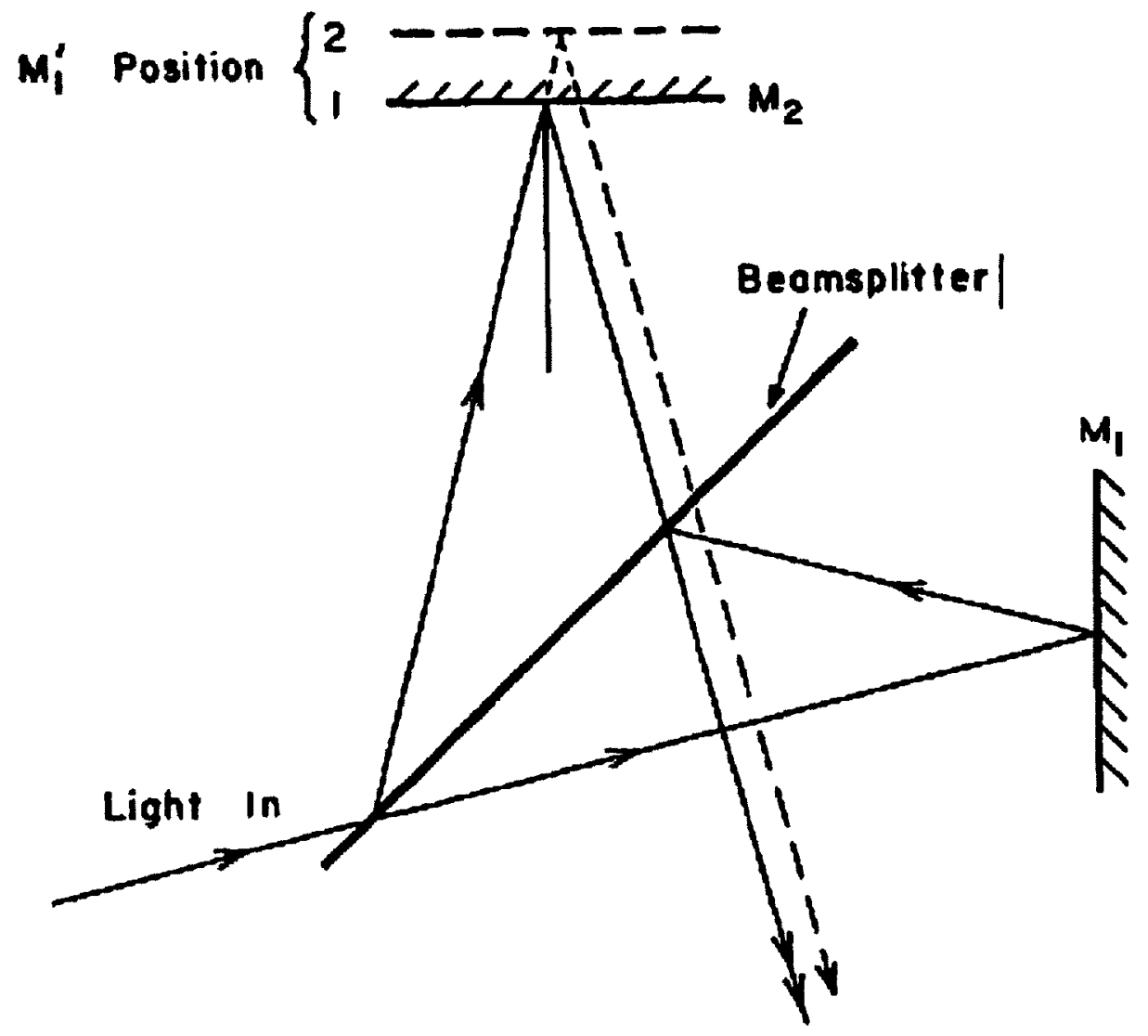

Figure 5.4: Micheison interferometer, where the incoming light is split with a beam splitter. $50 \%$ of the light is sent to the sample $\left(\mathrm{M}_{2}\right)$, while the remaining $50 \%$ is reflected of a mirror $\left(M_{1}\right)^{201}$. The change in frequency between $M_{1}$ and $M_{2}$ is compared, which gives an indication of the change in frequency of motion. Considering the same reduced mass, the changes in vibrational motion ( $k$ in Figure 5.4 ) which are characteristic of different chemical environments (at resonance) will give rise to diagnostic vibrational frequencies of motion.

The intensities of the peaks (in turn peak areas) at various wavenumbers correspond to the amount of those chemical bonds present in the sample. Thus, by making a calibration curve, FTIR can be used for quantitative analysis to determine concentrations of unknown molecules, provided there is a sufficient difference in vibrational 
frequencies of the chemical bonds (little overlap) and little interference from the solvent.

In this chapter, we have identified the primary amine wavenumber in L-lysine and attempted some quantitative analysis to determine concentrations. We could only measure the concentrations qualitatively or semi-quantitatively at best, since our solvent (water) interferes with the absorbance region of L-lysine and it cannot be quantitatively subtracted.

\subsubsection{Lysine}

In this chapter, we focused on a new target, Lysine. Lysine, (2,6-diaminohexanoic acid) is a basic amino acid (Figure 5.5 (a)) found in a myriad of biological organisms. Compared to the other basic amino acids, it contains a protonated alkyl amino group as its side chain under physiological conditions.
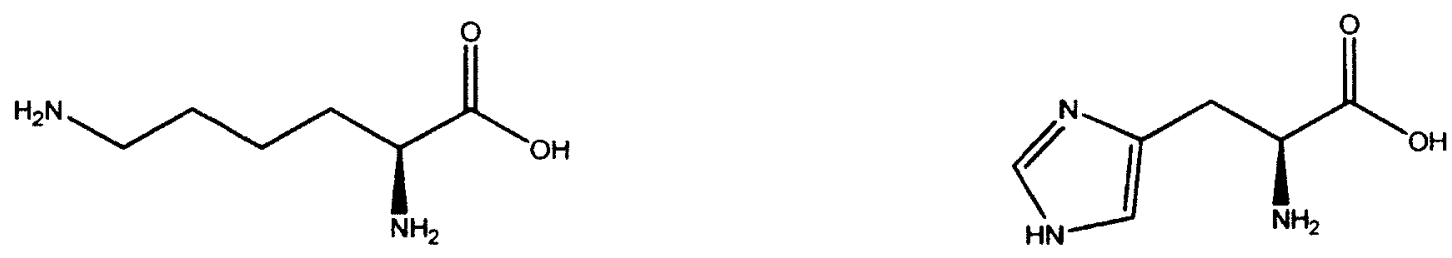

Figure 5.5: Structures of (a) L-lysine [left]; and (b) L-histidine [right].

Lysine plays an important role in protein electrostatic interactions. Histidine (Figure 5.5 (b)), another basic amino acid with an imidazolium side chain, is only $10 \%$ protonated at physiological conditions and plays a role in enzyme reactions ${ }^{202}$. Similar to the other amino acids, the L chiral form of lysine is the typically found in nature. 
In our study lysine was chosen because it is implicated as an exudate signal from the crop, possibly linked to stimulated nutrient release from microbial communities endogenous to the soils. Signaling molecules during crop growth have been identified in the past. Chandrashekaran ${ }^{203}$ studied the effect of microbial communities in the soil rhizosphere (close to plant roots) at different times of crop growth. He found an increased production of four major organic acids, part of the citric acid cycle, during stages of crop growth. The organic acids were being produced by microbes in the rhizosphere to help support breakdown of nutrients in the soil for uptake. A difference in soil conditions, such as $\mathrm{pH}$ and ionic strength, was also noted, thus, suggesting a stimulatory change to promote nutrient release. Osterberg and co-worker ${ }^{204}$ studied the effect of amino acid production in soils under different conditions (plowed and unplowed). They found evidence of various amino acids in the soils, bound to humic substances, under the two conditions. Lysine was an amino acid identified by the group in the un-plowed soil samples, however, the concentration was the lowest relative to the other amino acids present. The group did not suggest any reasoning or the mechanism for Lysine presence in the soil samples. It is possible that the lysine presence is purely a result of lysine present in microbial cell structure endogenous to soils. However, this suggests stability of this amino acid in the soil, thereby, making it attractive for our smart fertilizers since we do not want ready biodegradation of our signaling molecule before it reaches its target. Collaborators at Agriculture and Agrifood Canada have evidence that lysine resides in soil solutions to promote nutrient formation and subsequent uptake (personal communication with Dr. Carlos Monreal). Our 
collaborators have tracked and found that lysine content varies with crop growth stages. While the reason for this relationship still remains under discussion, it is a possible target for our aptamer to bind to and release the nutrients in our smart fertilizers.

\subsection{Experimental}

\subsubsection{Materials}

L-lysine (>98\%), L-histidine (98\%) were purchased from Sigma-Aldrich. All other reagents were purchased as already described in previous chapters and used without further purification.

\subsubsection{Methods}

Lysine aptamer: fluorescein (FITC) labeled aptamer for lysine was synthesized the same way as the sulforhodamine B aptamer (Chapter 2). The sequence was:

ATACCAGCTTATTCAATTTGAGGCGGGTGGGTGGGTTGAATACGCTGATTACCCCATCGGAGAA CGTTAAGGCGCTTCAGATAGTAAGTGCAATCT-FITC ${ }^{205}$. In brief, the aptamer was synthesized on a MerMade 6 DNA synthesizer (Bioautomation Corporation) using standard phosphoramidite chemistry ${ }^{206}$ on $1 \mu$ mol controlled pore glass (CPG) beads. The aptamers were hydrolyzed off the CPG beads in $48 \%$ ammonium hydroxide solution (v/v) for 48 hours at $45^{\circ} \mathrm{C}$. The aptamer was purified on $12 \%$ poly(acrylamide) gel electrophoresis for 1.5 hours at $45 \mathrm{~mA}$ and $1000 \mathrm{~V}$. The fluorescent broad UV 
fluorescence)/absorbance (at $260 \mathrm{~nm}$ ) band was collected and used as is. All glassware was rinsed $5 X$ with distilled and $5 X$ with deionized water).

Binding Affinities: Dissociation constant $\left(K_{d}\right)$ was evaluated using fluorescence anisotropy FTIR. Anisotropy: For anisotropy, a range of L-lysine solutions in $0.1 \mathrm{M} \mathrm{KCl}$ covering a range from $1 \times 10^{-3} \mathrm{M}$ to $1 \times 10^{-11} \mathrm{M}$ were prepared (by serial dilution ensuring $0.1 \mathrm{M} \mathrm{KCl}$ concentration) while keeping a constant aptamer concentration of $3.79 \times 10^{-}$ ${ }^{5} \mathrm{M}$ (UV/vis; Cary 300 ) in solution (1:1 dilution of L-lysine:DNA). The aptamer was denatured at $70^{\circ} \mathrm{C}$ for 20 minutes and cooled to RT before mixing with L-lysine. In the first set of studies the incubation time of aptamer and target was ca. 5 minutes, while in the second study we extended the incubation time to ca. 24 hours. Fluorescence anisotropy was measured using exc/emi wavelengths of $495 / 525 \mathrm{~nm}$ with a slit width for exc/emi of $4 \mathrm{~nm}$ with an acquisition time of 1 second in a $40 \mu \mathrm{L}$ cell. FTIR analysis was performed by mixing the solutions at same concentrations as used for anisotropy and filtering each dilution through a desalting tube (Amicon Ultra Cetrifugal Filters $0.5 \mathrm{~mL}$ with $3 \mathrm{~K}$ membrane at 10,000 XG (Thermo Sorvall Micro21 Centrifuge) for 30 minutes. Samples collected before incubating with aptamer and the filtrate after desalting were run on the FTIR (ABB Bomem MB Series). Samples were prepared by dispersing $50 \mu \mathrm{L}$ between two $\mathrm{KCl}$ plates (including water as background).

Microcapsules: All microscopy was performed in solution at RT. Microcapsules were prepared the same way as described in Chapter 3 using the same PSS doped $\mathrm{CaCO}_{3}$ cores and FITC modified L-lysine aptamer. The microcapsules were characterized on the 
confocal microscope (Zeiss CLSM 510) by looking at the fluorescein channel (489nm laser at $10 \%$ laser intensity for fluorescein) for the modified aptamer. Slight modifications were made to the protocol, where $0.8 \mathrm{M} \mathrm{NaCl}$ was used in the PAH, PSS, and aptamer solutions as supporting electrolyte, compared the $0.5 \mathrm{M}$ used in Chapter 3. FRAP was performed as described in chapter 3 using sulforhodamine B dye $(100 \mu \mathrm{M}$; $561 \mathrm{~nm}$ laser at $\mathbf{5 - 1 0 \%}$ laser intensity) as the fluorescent dye for measurements. The aptamer was not tagged with fluorescein (FITC) for FRAP. FRAP was performed on samples containing $3 \mu \mathrm{M}$ L-lysine $(0.1 \mathrm{M} \mathrm{KCl})$ as target and $3 \mu \mathrm{M}$ L-histidine $(0.1 \mathrm{M} \mathrm{KCl})$ as negative control and $100 \mu \mathrm{M}$ sulforhodamine $\mathrm{B}$ as the fluorescence signal. Annealing was done at $70{ }^{\circ} \mathrm{C}$ for 5 minutes.

\subsection{Results and Discussion}

\subsubsection{Dissociation Constant $\left(K_{d}\right)$}

After successfully demonstrating the ability of our model system to be specific, sensitive and have the ability to change diffusion upon aptamer-target binding (Chapter 1-3), we wanted to test our model using an aptamer which is a possible target candidate for our smart fertilizer. As already discussed, we identified lysine as a possible root exudates model compound. There is no aptamer produced specifically for lysine. Strehlitz and coworkers $^{207}$ discovered an aptamer binding to ethanolamine. The aptamer binds in a Gquadruplex conformation to ethanolamine based on the presence of G-rich regions in the aptamer. 
Strehlitz and co-workers ${ }^{208}$ have more recently tested their ethanolamine aptamer for binding specifically to other amine containing compounds. They found that the aptamer binds to various amino containing compounds, including amino acids. The aptamer has specificity towards alkyl amines (such as L-lysine) but not towards heterocyclic amines (such as L-histidine). This provided a starting point for this project as we wanted to test if diffusion can be altered, using FRAP, by aptamers for small molecules (amino acids). The aptamer sequence in this case (see Experimental) is $3 \times$ longer than the sulforhodamine B aptamer. Strehiltz and co-workers in their study did not establish a dissociation constant $\left(K_{d}\right)$ for the ethanolamine aptamer towards L-lysine. To optimize the FRAP experiments for maximum binding affinity, we started by measuring the $K_{d}$ of the aptamer towards L-lysine. As can be imagined it is very difficult to measure Kd's of aptamers for small molecules due to the subtle changes in signal measurement. We first attempted to evaluate the $K_{d}$ by FTIR using desalting tubes (Figure 5.6).

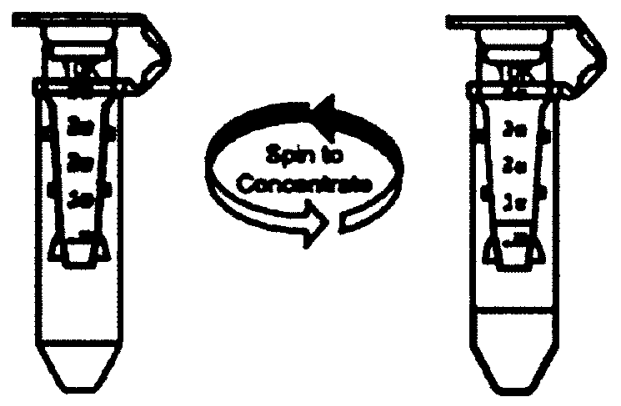

Figure 5.6: Desalting/filtration tubes where the solution is spun in a centrifuge and materials larger than the filter molecular weight cut-off is retained while all smaller molecules are eluted ${ }^{209}$. Some non-specific binding to the filter itself is expected during filtration. The image was adapted from the manufacturer for desalting tubes used in our laboratory. 
Our hypothesis was that if there was a change in resultant concentration of L-lysine in the filtrate as a result of change in aptamer-target binding, this could be measured quantitatively by FTIR and a $K_{d}$ determined. Solutions containing a L-lysine concentration range of $1 \times 10^{-3}$ to $1 \times 10^{-11} \mathrm{M}$ in $0.1 \mathrm{M} \mathrm{KCl}$ (to stabilize the G-quadruplex) were mixed with equivolume DNA $\left(3.79 \times 10^{-5} \mathrm{M}\right.$; quantified using Figure $4.8 \lambda_{260}$ and $\varepsilon=$ 1028000). Prior to mixing with target (and quantifying), the aptamer solution was heated at $70^{\circ} \mathrm{C}$ for 20 minutes to denature the strand.

After mixing, the solutions were incubated for five minutes and filtered on the desalting tubes for 30 minutes at $10,000 \times G$ under RT. FTIR spectra were collected for L-lysine in $0.1 \mathrm{M} \mathrm{KCl}$ before mixing with the aptamer and after desalting (filtrate). The spectra were collected in aqueous samples, and a water background $(50 \mu \mathrm{L})$ was used to remove water interference on the peak of interest. To remove non-specific binding of L-lysine to the filter, measurements were also made on eluting the L-lysine solution in the absence of the aptamer and measuring the non-specific binding to filter. The integrated area under the peak centered at $1600 \mathrm{~cm}^{-1}$ (from 1500 to $1800 \mathrm{~cm}^{-1}$ ) was determined from the raw data (Figure 5.7(a), which corresponds to a primary amine bend ${ }^{210}$. Before and after values were subtracted and fit to the $K_{d}$ equations already described in Chapter 1. Using FTIR we were unable to determine a convincing fit to the data. By removing four experimental points, we were able to estimate qualitatively a possible $K_{d}$ in the $\mu \mathrm{M}$ concentration (see Figure 5.7(b)). The trend is consistent with a binding curve showing that as the concentration of target is increased, a higher quantity of molecules are 
bound to the aptamer and thus the quantity eluted through the filter decreases. Since only one data point falls on the decreasing portion of the curve, a $K_{d}$ cannot accurately be determined. The FTIR data can only be used as qualitative evidence that a $K_{d}$ may fall somewhere in the $\mu \mathrm{M}$ range based on the large change in signal at those concentrations. 

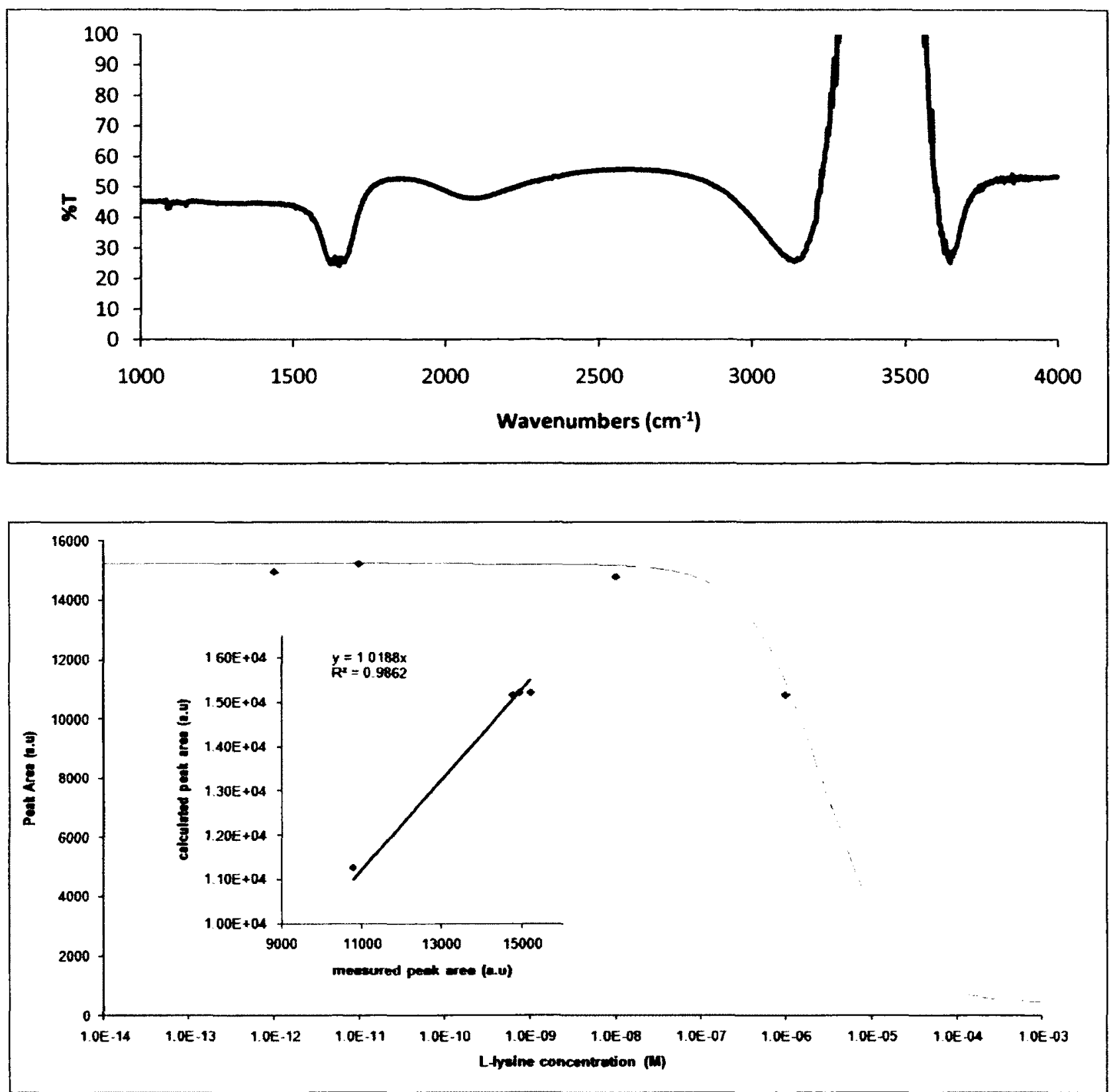

Figure 5.7: (a) raw FTIR spectrum of filtrate after aptamer-target incubation and subtraction of solvent background (water). Peak centered at ca. $1600 \mathrm{~cm}^{-1}$ was considered to be from the primary amine. Percentage area was determined for the amine peak by using FTIR software. (b) $K_{d}$ data fitting for the aptamer by removing four data points with the correlation between predicted and observed data shown in the inset. Figure (b) shows a rough approximation of where a $K_{d}$ might lay. As the concentration is increased of the target, less quantity of target (L-lysine) is eluting through the filter until there is a significant decrease in quantity of target eluted ca. $2 \mathrm{x}$ $10^{-6} \mathrm{M}$ which could be an expected $K_{d}$. 
The aptamer was recovered after FTIR analysis by repeated desalting/filtration steps with $20 \mathrm{~mL}$ of deionized water (at $80^{\circ} \mathrm{C}$ ) to denature the DNA, thereby, allowing L-lysine to be filtered as it is diluted. Using the same concentrations (of aptamer and L-lysine), we next tried fluorescence anisotropy. As was done with the sulforhodamine B aptamer, we incubated the samples for ca. fifteen minutes and measured anisotropy. Anisotropy of just solvent (i.e., water) was subtracted as background. Using such conditions did not yield any meaningful data from which a $K_{d}$ could be extracted (Figure 5.8). Looking at Figure 5.8 , a $K_{d}$ can only be visually estimated to be somewhere between $1 \times 10^{-7}$ to $1 \mathrm{x}$ $10^{-6} \mathrm{M}$ based on the large decrease in absolute anisotropy, in line with the estimation made from FTIR; however, the data was noisy.

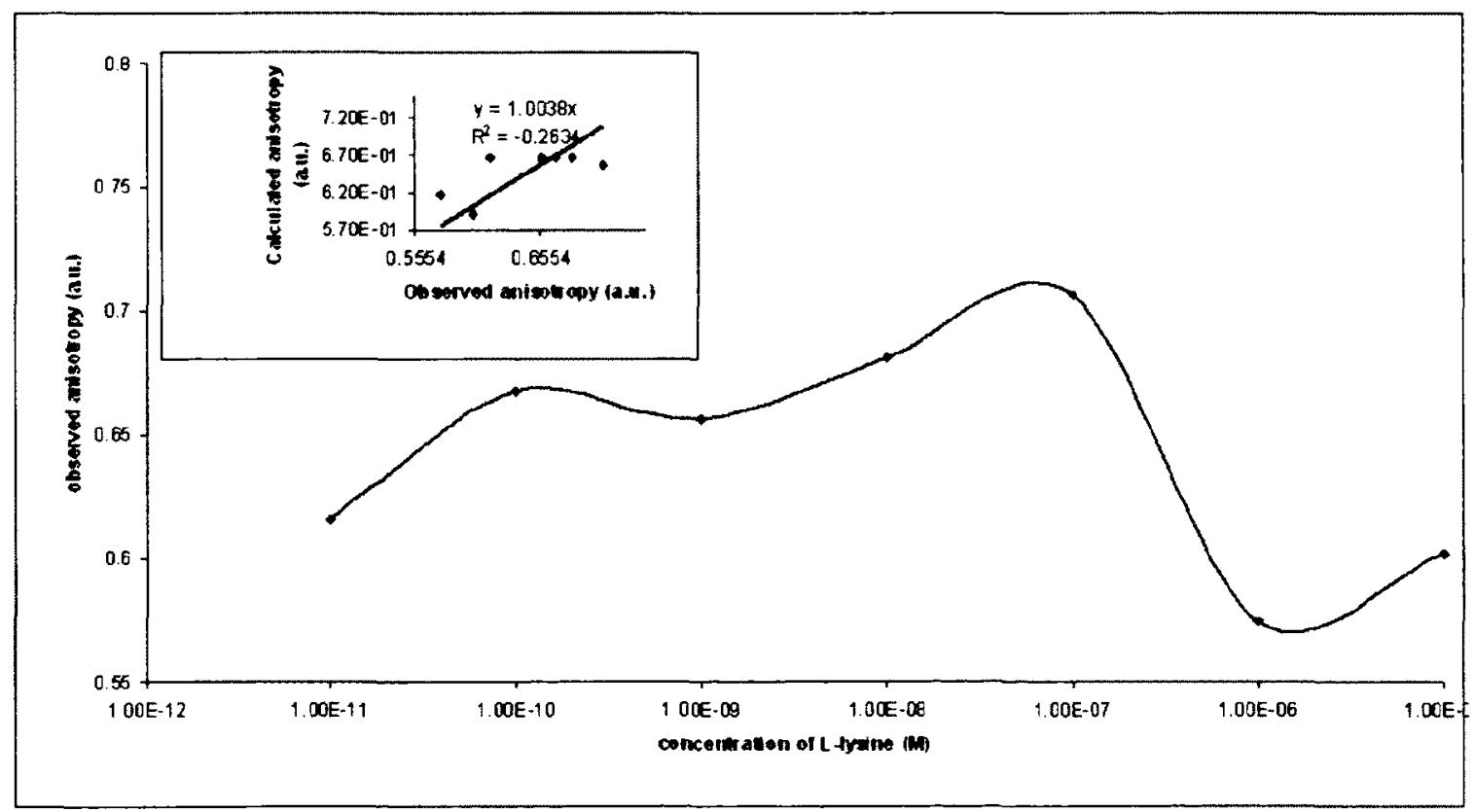

Figure 5.8: Anisotropy data shown after ca. fifteen minute aptamer-target incubation in $0.1 \mathrm{M} \mathrm{KCl}$, with the correlation between observed and calculated anisotropy's in the inset. 
incubation. The purpose of this experiment was to optimize the concentrations used for FRAP experiments and not to definitively measure a $K_{d}$ for this aptamer with L-lysine. As such, we did not feel any need to gather more data points at higher concentrations. There is a decrease in anisotropy at higher concentrations, which is counter intuitive as the anisotropy should increase as more molecules are bound and the rotational diffusion slowed. A possible theory for this trend is that as our tagged aptamer folds upon binding to its target, the rotational diffusion goes up instead of down as it folds into a tight conformation relative to the linear-like native strand before binding, thereby, decreasing the overall anisotropy. It is clear from the anisotropy data that increasing the incubation time, increased sensitivity and reproducibility of our data. Thus, we found evidence between incubation time and determination of a dissociation constant, possibly specific to G-quadruplex formation. We hypothesize that this difference in the need of longer incubation time related to the differences in size between this aptamer and the sulforhodamine B aptamer. The larger aptamer may require increased time to form a stable G-quadruplex. Time-dependant G-quadruplex studies have been shown in literature by performing imino proton NMR spectra. As an example, Patel and co-workers studied the G-quadruplex formation potential of human telomeric DNA in the presence of $\mathrm{K}^{+}$and found a stable G-quadruplex form of the DNA after $24 \mathrm{hrs}$ of incubation ${ }^{211}$. Thus, we believe that while the sulforhodamine B aptamer was able to easily stabilize in the presence of its target, the aptamer for L-lysine needed a longer residence time to stabilize, possibly due to steric or entropic considerations. 


\subsubsection{Diffusion Coefficient}

Next, we wanted to test the diffusion characteristics of our films embedded with the much larger aptamer (relative to Chapter 1-3). For comparison with our model system, we used the same conditions as Chapter 2 (FRAP), with slight modifications. The sacrificial PSS doped $\mathrm{CaCO}_{3}$ cores used were the same as those in Chapter 2. To confirm microcapsule presence, we labelled the aptamer with fluorescein. The cores were modified using $2 \mathrm{mg} / \mathrm{mL}$ PAH, PSS, and $3.79 \times 10^{-5} \mathrm{M}$ DNA in $0.5 \mathrm{M} \mathrm{NaCl}$ as described in Chapter 2. The $\mathrm{Ibl}$ deposition consisted of $(\mathrm{PAH})_{2}(\mathrm{DNA})_{2}(\mathrm{PSS})_{2}$ where the DNA was embedded between the films, same as in Chapter 2. After dissolution by EDTA, the microcapsules were imaged under the confocal microscope with the $489 \mathrm{~nm}$ laser $(10 \%$ laser output). We found no presence of microcapsules and saw a lot of collapsed material under the microscope (Figure 5.10).

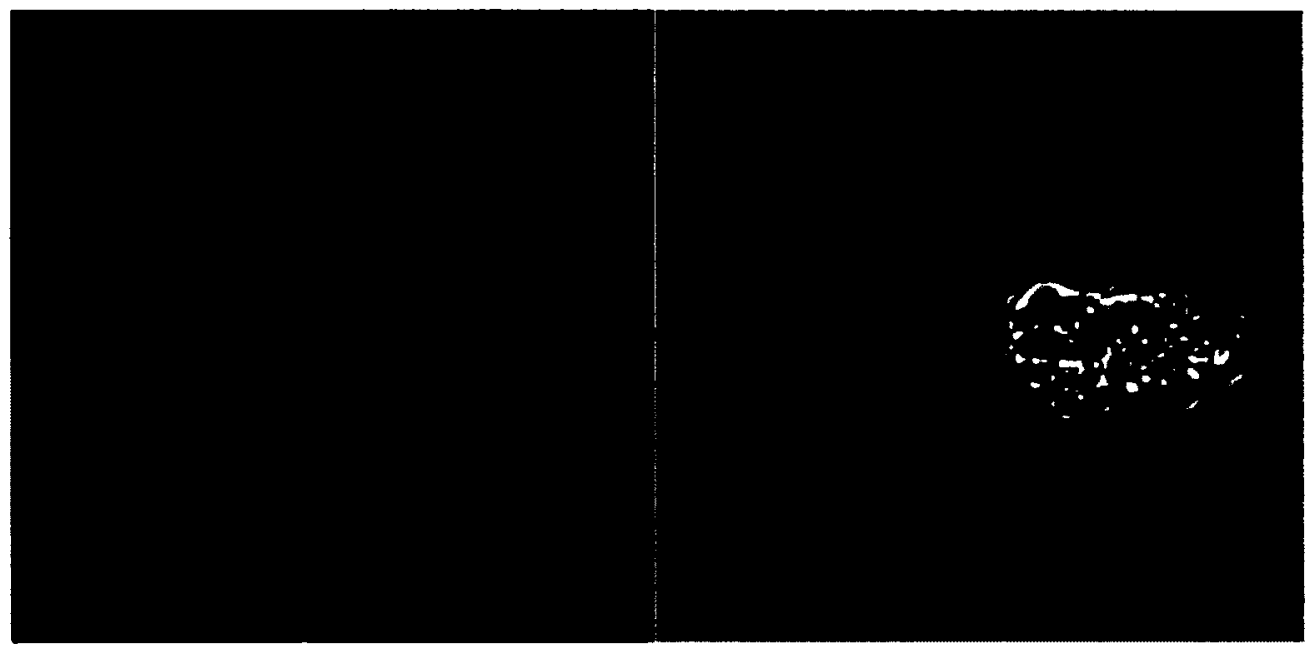

Figure 5.10: Confocal microscopy image after core dissolution with $0.5 \mathrm{M} \mathrm{NaCl}$ as supporting electrolyte. We found no presence of microcapsules in the fluorescence (left) or light (right) channels. Bars correspond to $10 \mu \mathrm{m}$. 
We attribute the failed microcapsules to high osmotic pressure and flexibility from the large aptamer strand. Effects of concentration and type of polyelectrolyte on osmotic pressure have been studied in detail by Bloomfield and co-workers who calculated the osmotic pressure of polyelectrolyte systems covering a decade of research ${ }^{212}$ using Scaling theory. Moreover, Mohwald and co-workers studied the effect of osmotic pressure on PAH/PSS microcapsules, and found that it depended on the concentration of polyelectrolytes incorporated in the microcapsule (polyelectrolyte concentration) and salt concentration (wall thickness) ${ }^{213}$. Their findings indicate a balance between concentration (of polyelectrolyte and salt) and wall thickness to form stable microcapsules. In the case of our microcapsules, using the smaller sulforhodamine B aptamer, there were more apatmer strands per layer which equated to a higher concentration of aptamer incorporated per layer. In the case of the L-lysine aptamer, the aptamer is c a. $3 X$ larger than the sulforhodamine B aptamer, and thus qualitatively, one can estimate three times lower concentration of aptamers incorporated into the bilayers. Using this argument, we believe that the lower concentration was unable to sustain the osmotic pressure and the capsules fell apart because the walis were thinner and the charge density was lower. While we would be unable to increase the aptamer concentration, without significantly increasing the capsule size, we increased the $\mathrm{NaCl}$ concentration from 0.5M to $0.8 \mathrm{M}$ to increase film thickness ${ }^{214}$ of PAH and PSS onto the $\mathrm{CaCO}_{3}$ core, thereby providing stronger rigidity to the overall structure. The only drawback of increasing the salt concentration is that the film morphology becomes less 
flat on the nanometer scale. We were successful in producing microcapsules using this approach (Figure 5.11(a) and (b)). The microcapsules had an average size of $\sim 6-7 \mu \mathrm{m}$ which is consistent with our previous microcapsules for the sulforhodamine B aptamer. The microcapsules as seen in Figure 5.11(a) were mostly spherical. Figure 5.11(a) also shows dark regions (black voids) inside the microcapsules indicating hollow capsules, since only the aptamer incorporated in the walls was tagged. Figure 5.11(b) suggests full microcapsules after aptamer-target binding with sulforhodamine $B$ dye, where the aptamers are not tagged with fluorescein. Just as in Chapter 3, in the following images green corresponds to fluorescein while yellow to sulforhodamine B. 


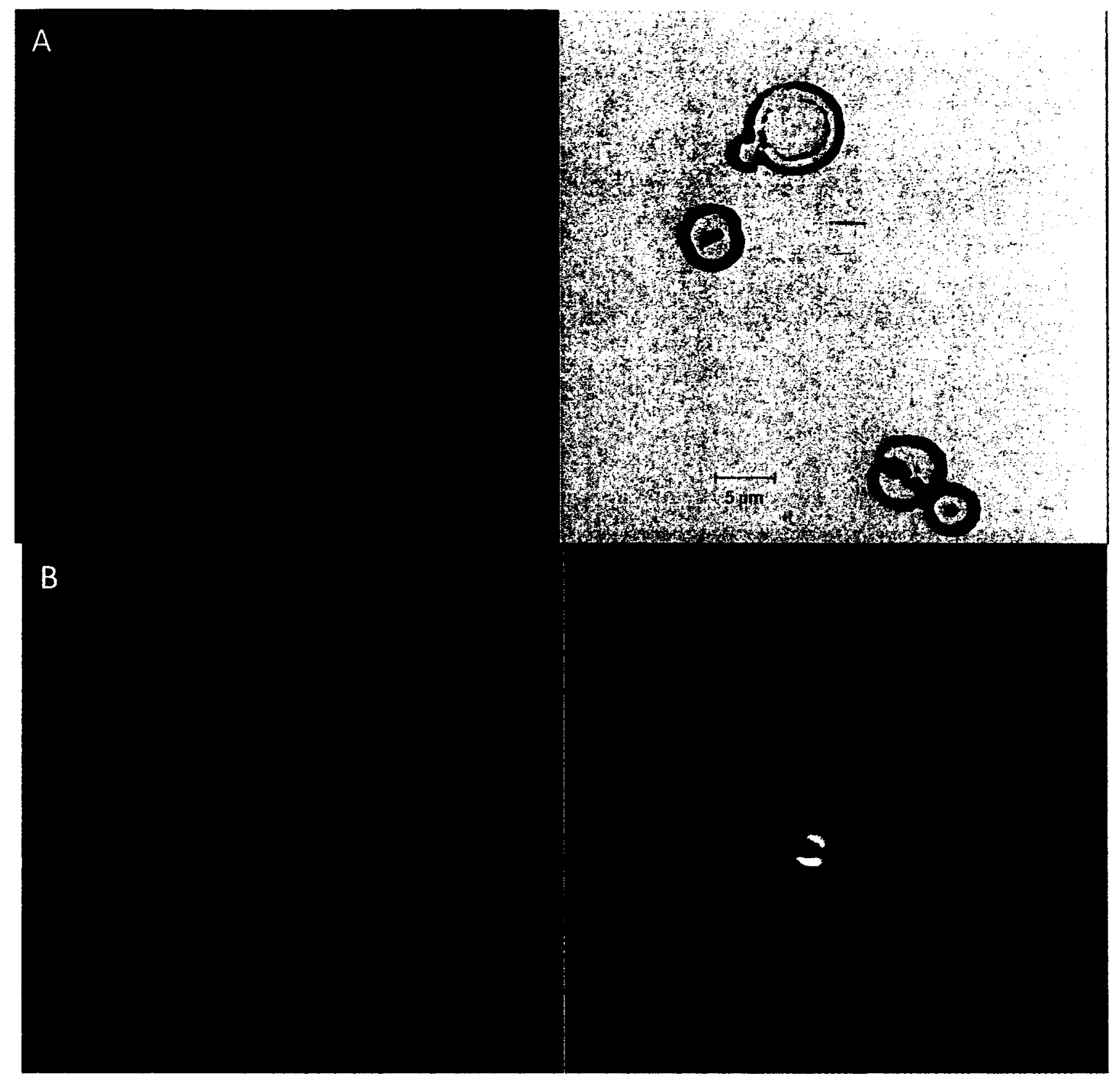

Figure 5.11: Confocal microscope image of: (a) microcapsules with fluorescein tagged aptamer (green; left) and light channel (right); and (b) microcapsules with filled sulforhodamine B (vellow; left) and light channel (right). Red bars are $5 \mu \mathrm{m}$.

We have previously (Chapter 3 ) studied effects of diffusion before and after annealing. In this case, annealing broke the microcapsules (images not shown), likely as a result of 
denaturing of the aptamer, thereby, we looked at diffusion only before annealing. The FRAP measurements were made as previously described and analyzed according to the equations used in Chapter 3. A model FRAP experiment using these microcapsules (5.12(a)) and raw FRAP data for target L-lysine and control L-histidine, normalized only as relative percentage and not for bleach spot size, are shown in Figure 5.12(b). The data was fit using first order kinetics utilizing in-built microscope software (Kinetic). 

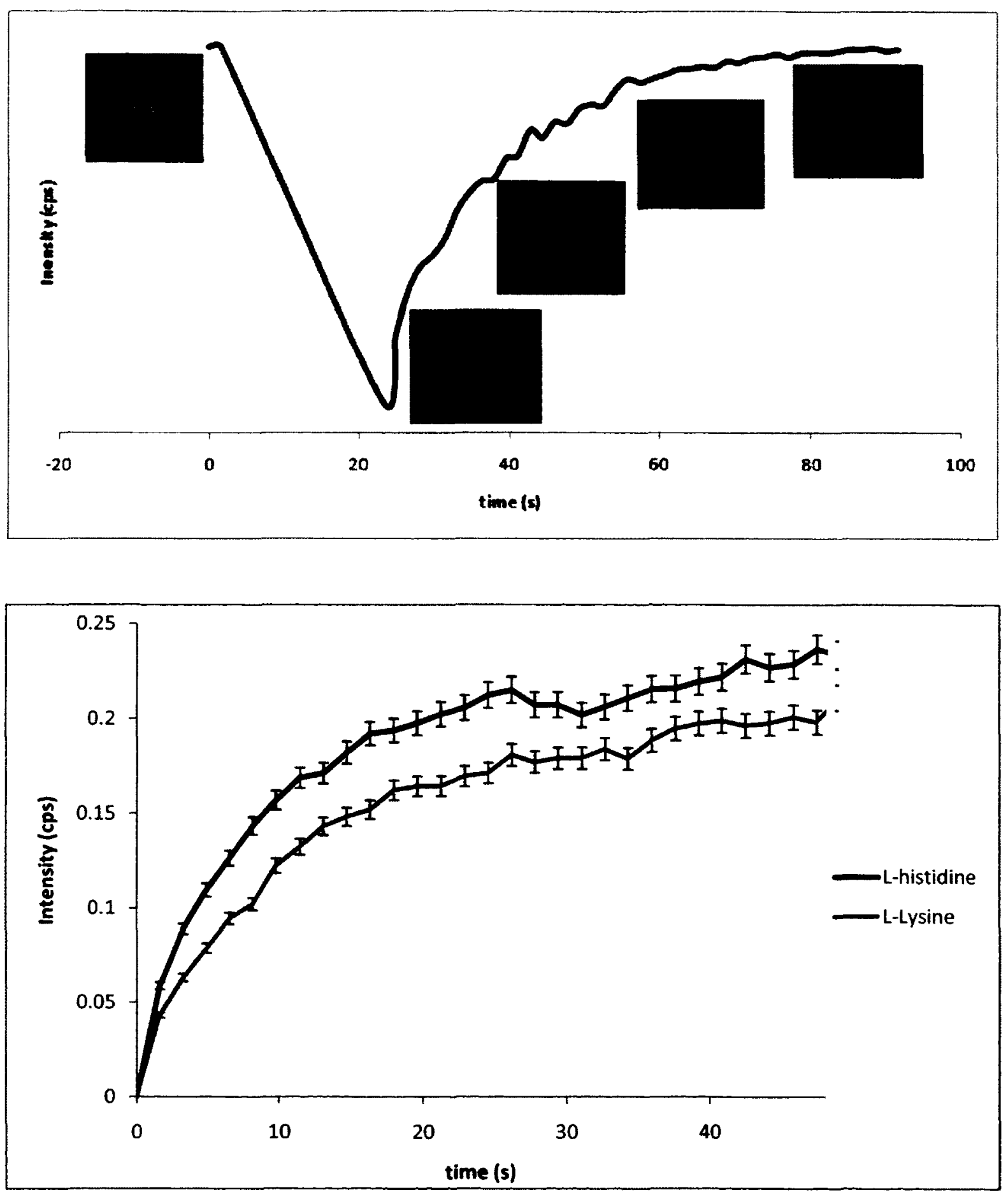

Figure 5.12: (a) Typical FRAP experiment in terms of intensity; and (b) raw data not normalized in terms of bleach spot size for L-lysine (black) and L-histidine (brown). While the raw data shows slower recovery of fluorescence after photobleaching, the bleach spot sizes were larger and thus once corrected for spot size, L-lysine had a diffusion ca. $2 X$ higher than L-histidine. 
The calculated diffusions for L-lysine and L-histidine (average over 7 microcapsules per sample) were found to $1.13 \times 10^{-14}$ and $5.07 \times 10^{-15}$ with standard errors of $4.26 \times 10^{-15}$ and $8.34 \times 10^{-16} \mathrm{~m}^{2} / \mathrm{s}$, respectively. We have found that the diffusion is ca. $2 \times$ higher upon aptamer-target binding relative to the negative control. This agrees with our findings discussed in previous chapters with the other microcapsules, values in literature (ca. $\sim 1 \times 10^{-15} \mathrm{~m}^{2} / \mathrm{s}$ at similar ionic strength for PAH/PSS microcapsules) ${ }^{215}$, and our electrochemistry findings. It is important to note that these diffusions differ from the ones from Chapter 3 in that these provide data on the diffusion of molecules, other than the target, through the microcapsule wall, compared to studying the diffusion of just target which was the case in Chapter 3. This data is consistent with the diffusion coefficient values found in our previous chapters and in literature.

Thus, if discussed together, it is apparent that aptamer binding can induce an increase in diffusion of not just the target but various other molecules or varying sizes, thereby, suggesting a very attractive mechanism for systems such as our smart fertilizer and possibly drug delivery. As an incremental step towards our smart fertilizer, this L-lysine system suggests a possible in situ system in greenhouses to test the efficacy of these model systems. As has also been shown, the wall thickness can easily be manipulated to target different diffusion profiles.

For future experiments, the wall thickness can further be manipulated to develop microcapsules which can collapse upon aptamer-target binding. This requires only the careful tailoring of the salt and polyelectrolyte concentrations to develop a system 
which will fall apart as a target diffuses through the wall. This could be very attractive for quick release systems, such as in our case with the fertilizers or drug delivery where instant relief is needed (such as after surgery or during amputation).

\section{$\underline{5.4 \text { Conclusions }}$}

We have designed, herein, a possible in-situ system for our smart fertilizers by using a relevant target, L-lysine. We have shown a ca. two times increase in diffusion profiles compared to the negative control. Taken together with our other findings, we have demonstrated that aptamer-target binding in microcapsules will change diffusion of not only the target, but molecules of varying sizes and charges.

\subsection{Acknowledgements}

Y.S thanks Tony O'Neil for help with FTIR data analysis. 


\section{Chapter 6. Nano-aggregates}

Sultan, Y.; Abboud, J.; Won, A.; Walsh, R.; lanoul, A.; DeRosa, M. under preparation

\subsection{Introduction}

\subsubsection{Biological superstructures}

Recently, there has been increasing interest in the incorporation of aptamers into supramolecular assemblies (superstructures, such as micelles and liposomes) for the development of hybrid systems that combine the binding affinity and specificity of the aptamer with the unique physical, chemical and biological properties provided by these amphiphilic self-assembling structures. For example, dialkylglycerol-conjugated aptamers have been incorporated into liposomes in order to improve the aptamer's plasma residence time $\mathrm{e}^{216}$. Aptamers modified with cholesterol ${ }^{217}$ or polyethylene glycoldistearoylphosphatidylethanolamine ${ }^{218}$ have been inserted into liposomes, exploiting the ability of liposome to serve as an encapsulation agent, in order to create targeted drug delivery systems. Similarly, aptamers have been covalently tethered to the surface of drug-loaded polymer nanoparticles ${ }^{219}$ yielding targeted therapeutic systems. More recently, lipid-modified aptamer micelles with improved target binding affinity over unmodified aptamers have been developed for cancer cell recognition and drug delivery applications ${ }^{220}$. Aptamers have also been conjugated to polymeric micelles for the development of a turbidometric sensor ${ }^{221}$. 
In this chapter, we report on a simple and general synthesis strategy for preparing alkylaptamer conjugates capable of self-assembling to form nano- and micro-structured aggregates with molecular recognition properties defined by the aptamer sequence. The sulforhodamine B aptamer ${ }^{222}$ was used as our model system for this study. Our data shows that these aggregates had a dramatically improved apparent binding affinity compared to the unmodified aptamer and unaggregated alkyl-aptamer conjugates. This opens up new possibilities for the development of tunable, high-affinity hybrid aptamer particles for the ultrasensitive detection of targets and for smart drug delivery or our smart fertilizers. Characterization was done by Atomic Force Microscopy (AFM), SEM, and the apparent dissociation constant $\left(K_{d}\right)$ was determined by fluorescence anisotropy, as previously described. For our conjugates, we prepared an amphiphilic molecule to mimic biological molecules.

\subsubsection{Hydrophobic Forces}

The hydrophobic effect is, by and large, one of the most important driving forces for forming superstructures of biological molecules ${ }^{223}$. For simplicity, the following discussion will only look at the effect of hydrophobic forces in water. An amphiphilic molecule contains a hydrophile (head) and a hydrophobe (tail), typically polar and nonpolar group regions. The hydrophile is attracted to water, while the hydrophobe is repelled by water molecules. Water molecules at equilibrium, according to the second law of thermodynamics, are in a state of maximum entropy $(\Delta S>0)$ and minimum Gibb's free energy $(\Delta G<0)$. When a solute is added to water, $\Delta S$ and $\Delta G$ are decreased. In the 
case of a hydrophile, this loss of entropy is compensated by hydration between the broken hydrogen bonds of water and the charged molecule, thus re-maximizing free energy. In the case of a hydrophobe, there is no compensation in free energy by association; rather the solute is repelled to compensate the entropic and free energy loss. In the case of an amphiphile, these two effects happen concurrently. When an amphiphile is added to water, the loss of entropy (via loss of hydrogen bonds) is partially compensated by hydration of the head region, while the tail is repelled. This repulsion at each hydrophobic molecule is a function of interstitial stress which is in turn a function of the amount molecules of water trying to repel the molecule. This process drives the formation of bilayers, micelles, aggregates, etc, superstructures where the hydrophobic region has self-associated minimize the entropic loss of water. Noteworthy is that hydrophobe-hydrophobe repulsion has little effect on the structure formed ${ }^{223}$. Chemically, the formation on the type/size of superstructures depends primarily on (1) type of hydrophile, i.e., degree of hydration possible to compensate for the entropy loss of hydrophobe; (b) hydrophobe length and type, i.e., amount of interstitial stress felt as an increase of molar volume of water; and (c) concentration of water, i.e., amount of repulsion due to the entropic cost of increasing water concentration. For example, a large hydrophile and small hydrophobe will need a higher molar fraction of water to form superstructures compared to large hydrophobe and small hydrophile. Moreover, physical conditions, such as temperature and $\mathrm{pH}$ also affect the formation kinetics of these structures in water. Considering an amphiphile in water, at a low concentration of water, bilayers capsules are usually formed where the hydrophobic tails pack together 
and there is hydration association inside the void and outside on the surface by protruding hydrophile regions with water trapped inside the void. While at a high concentration of water, the interstitial stress is increased to the point that the hydrophobe repels all the water molecules and forms a micellar structure where the hydrophobe packs tightly while the hydrophile associates with surface molecules. In between bilayers and micelles, many different structures can be formed. The stability of an amphiphilic superstructure is related to the interaction energy between the amphiphile molecule and water (Equation 1), which is a function of various chemical and physical conditions. This value is typically reported as the critical micelle concentration (CMC). For most new amphiphiles, the CMC (or $n$ ) cannot be calculated based on theoretical data and a phase diagram of molar fraction (f) vs. polymer concentration needs to be developed which indicates the various morphologies ${ }^{224}$.

$$
C M C \sim \exp \left(\frac{n \varepsilon_{\mathrm{h}}}{K_{\mathrm{b}} T}\right)
$$

Where $K_{b} T=$ thermal energy, $\varepsilon_{h}=$ amphiphile's effective interaction energy with water, and $n=$ aggregate stability which is determined experimentally and is loosely labeled as the $\mathrm{CMC}^{224}$.

Figure 6.1 shows a typical phase diagram with the different structures possible. 

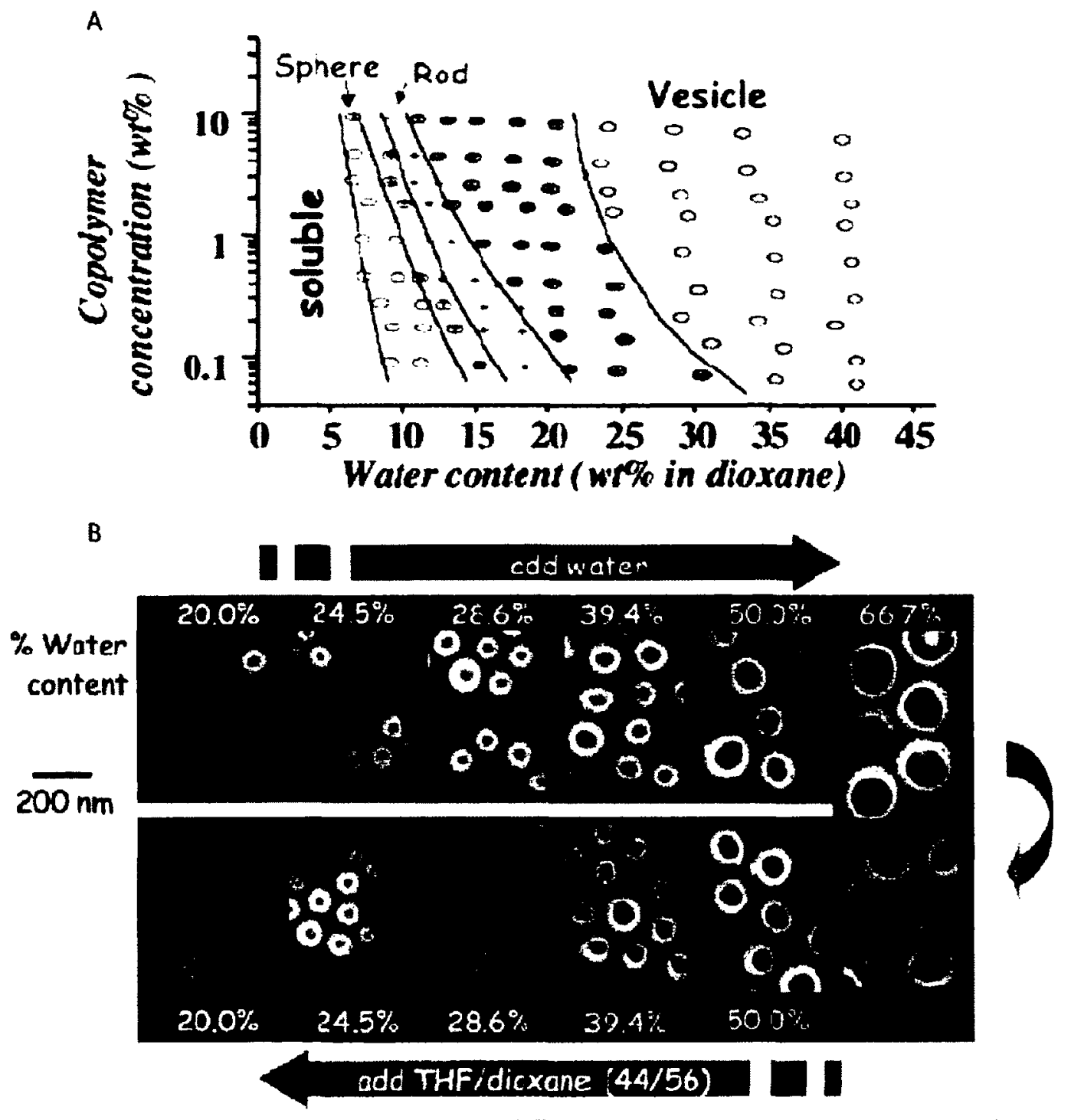

Figure 6.1: Phase diagram for synthetic polymeric amphiphiles. Phase diagrams are shown as a function of amphiphile concentration vs. water (a) and amphiphile solvent vs. water (b). Both phase diagrams shown that by increasing the molar concentration of water, the change in interstitial stress and hydration drives the formation of different morphologies ${ }^{225}$.

\subsubsection{Receptor Clustering}

We wanted to probe if our aggregates had size-dependant apparent $K_{d}$ trends. That is, whether there was a receptor clustering effect of our receptor (aptamer) to the ligand 
(target). Since we were unable to identify 1:1 stoichiometry between the aptamer and target, we refer to the $K_{d}$ as an apparent binding dissociation constant. Receptor clustering is seen in many biological systems. Hall and co-workers ${ }^{226}$ report that the complex postsynaptic structures of the adult neuromuscular junction are maintained by oligomerization (clustering) of acetylcholine receptors, signaled by agrin and laminin ligands. According to the group, the exact mechanisms and reasons for the clustering remain unknown, although it may possibly be to control a low concentration of ion flux. Further examples of receptor clustering have been documented by Kim and coworkers $^{227}$ and Germain ${ }^{228}$. The reason behind clustering is thought to be for improved sensitivity (lower $K_{d}$ ). Kim and co-workers have looked at the effect of receptor clustering on the behavior of Escherichia coli (E. Coli). Depending on the concentration of a specific receptor, the $E$. Coli bacterial cell swims straight or can tumble, which can in turn alter its behavior. Receptor clustering allows for low sensitivity of specific clusters which control the swimming behavior of E. Coli cells. Through modeling, the group was able to show that the clustering of the receptors on the E. Coli flagella allow for increased sensitivity for the ligand. As seen in Figure 6.2, there is a dramatic increase in sensitivity for a model ligand as the number of clusters is increased (solid line), while the sensitivity of single receptors (dashed line) is low for the same number of receptors on the surface ( $x$-axis $X 1000$ ). 


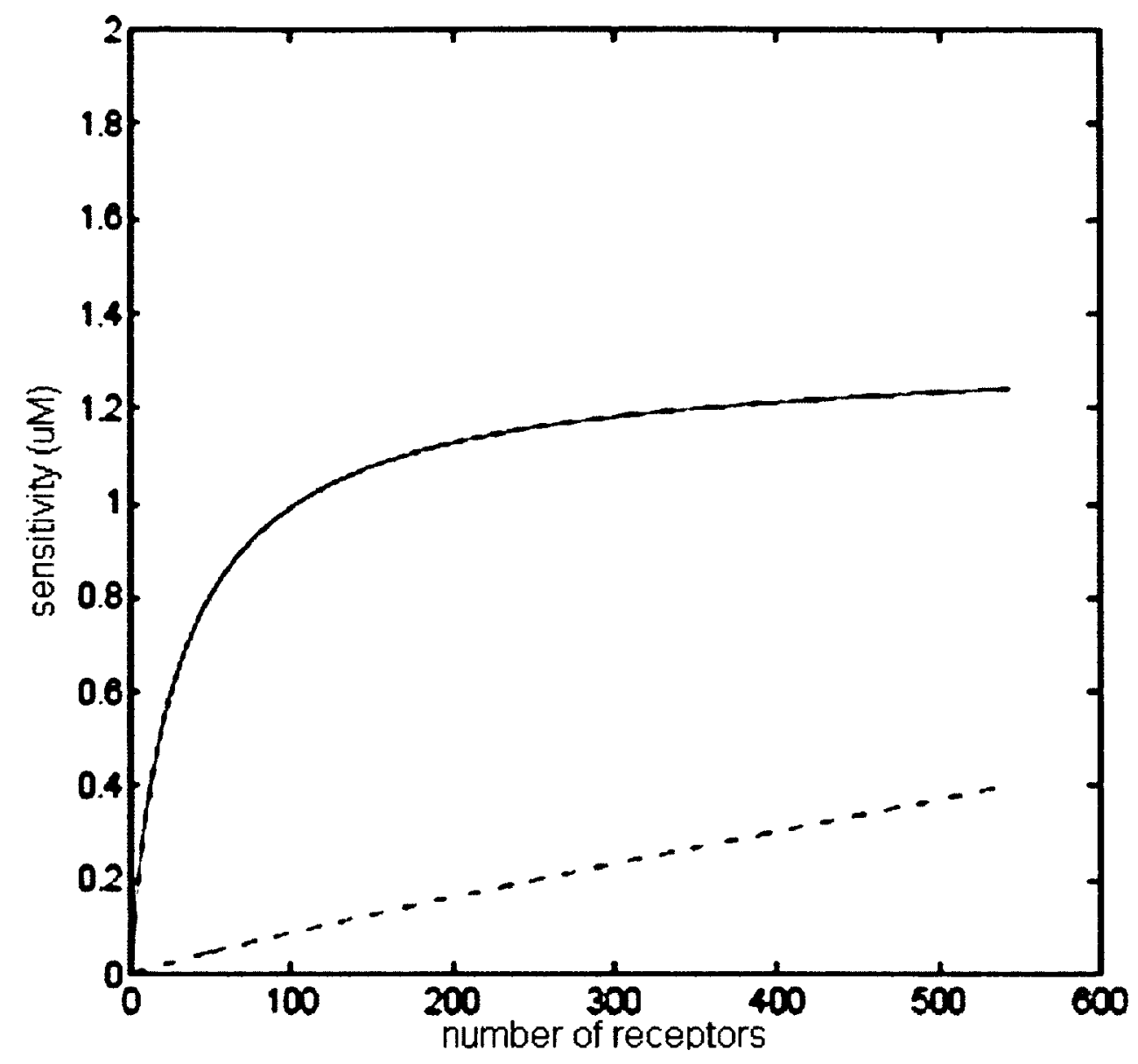

Figure 6.2: Effects of receptor clustering on sensitivity of detection ( $y$-axis) for clusters (solid line) and single receptors (dashed lines) dependant on the number of receptors ( $x$ axis $\times 1000)^{229}$. In this situation, the clusters are expected to be interconnected.

Morton-Firth and co-workers ${ }^{230}$ suggest that the increased sensitivity seen in receptor clustering is driven by activation/inactivation of neighboring receptors in a cluster. The group describes the principle pictorially according to Figure 6.3. 

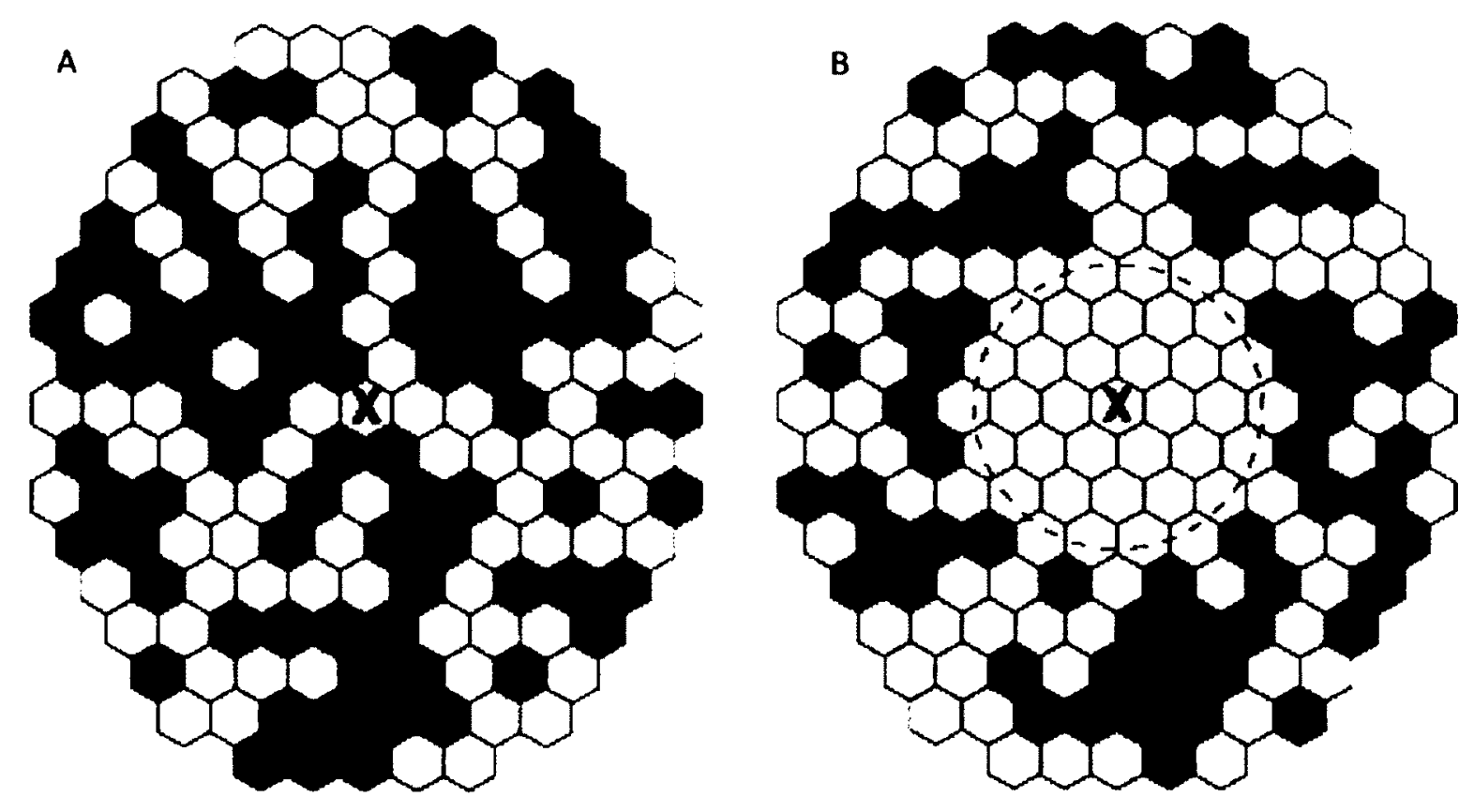

Figure 6.3: Receptor clustering model (theoretical): (a) a ligand (labeled $X$ ) is bound to an individual receptor (hexagon) and thus effects (activates/inactivates) only the one receptor, while (b) a ligand bound to one receptor effects a whole cluster (circle marked by dashed lines), thereby, increasing the detection sensitivity (signal strength). In the case of aptamers, an activated state is a free aptamer while inactivated states describe aptamers bound to targets. The white and grey hexagons refer to a random distribution of active and in active receptor sites used in modeling ${ }^{230}$.

As can be seen in Figure 6.3, receptor cluster lowers $K_{d}$ by increasing signal strength.

Consider a single receptor bound on a surface (Figure 6.3(a)), where the nearest neighbor is separated by a few nanometers, effectively not having any spatial affect on another receptor. In this case, the signal from the resultant ligand binding, whether it be chemical or analytical in nature, is driven by the single repector-ligand binding event. In the case of a cluster, where the receptors are physically in contact with each other (dashed line Figure 6.3(b)), receptor-ligand binding will have an effect on the whole 
cluster, thereby increasing the resultant signal strength. The minimum (i.e., sensitivity) and maximum (i.e., point of saturation) concentration change detectable can be calculated by:

$C_{\mathrm{A}}^{\min }=\left(\frac{\left(\frac{\Delta P_{\min }}{n_{1} \sigma}\right)}{N-\Delta p_{\min } / n_{1} \sigma}\right) K_{\mathrm{d}} \quad C_{\mathrm{A}}^{\max }=\left(\frac{\left(n_{\mathrm{A}}^{\max }\right)}{N-n_{\mathrm{A}}^{\max }}\right) K_{\mathrm{d}}$

Where $C_{A}{ }^{m i n} / C_{A}{ }^{\text {max }}=$ minimum $/$ maximum detectable concentration, respectively; $\Delta P_{\min }$ $=$ minimum change in activity of the cluster as a result of binding; $n_{1}=$ number of receptors effected by binding of single ligand; $n_{A}=$ number of receptors effected by ligand binding on cluster; $N=$ number of receptors in an array (cluster); $\sigma=$ change in activity per receptor; and $K_{d}=$ receptor-ligand dissociation constant ${ }^{230}$.

As can be seen from Equation 1, the minimum concentration $\left(C_{A}^{\text {min }}\right)$ is inversely dependant on the number of clusters affected by a single binding event $\left(n_{1}\right) \cdot C_{A}{ }^{\text {min }}$ is proportional to the $K_{d}$, in that the lower the minimum concentration of detection approaches, the lower the $K_{d}$. Thus, a cluster of $\mathrm{N}$ receptors can have a gradient of different $K_{d}$ 's dependant on the size of the cluster, thereby, allowing control of influx molecules in a biological system ${ }^{230}$. The theoretical response of detection (activity) as a result of varying number of clusters for the same number of receptor molecules can be seen in Figure 6.4. As can be noted, for a system with one big receptor cluster, that is, where a single ligand binding event effects the whole cluster will have the lowest $K_{d}$, while a system with four different clusters where each binding event will effect only $1 / 4^{\text {th }}$ the number of receptor molecules and thus will have the highest $K_{d}$ in the theoretical study. Also noteworthy is that as the extent of activity $\left(n_{1}\right)$ is increased, that 
is, increasing the number of receptors effects per cluster, the sensitivity is increased (lower $\left.K_{d}\right)$ in line with Equation $1\left(C_{A}^{\text {min }}\right)$.

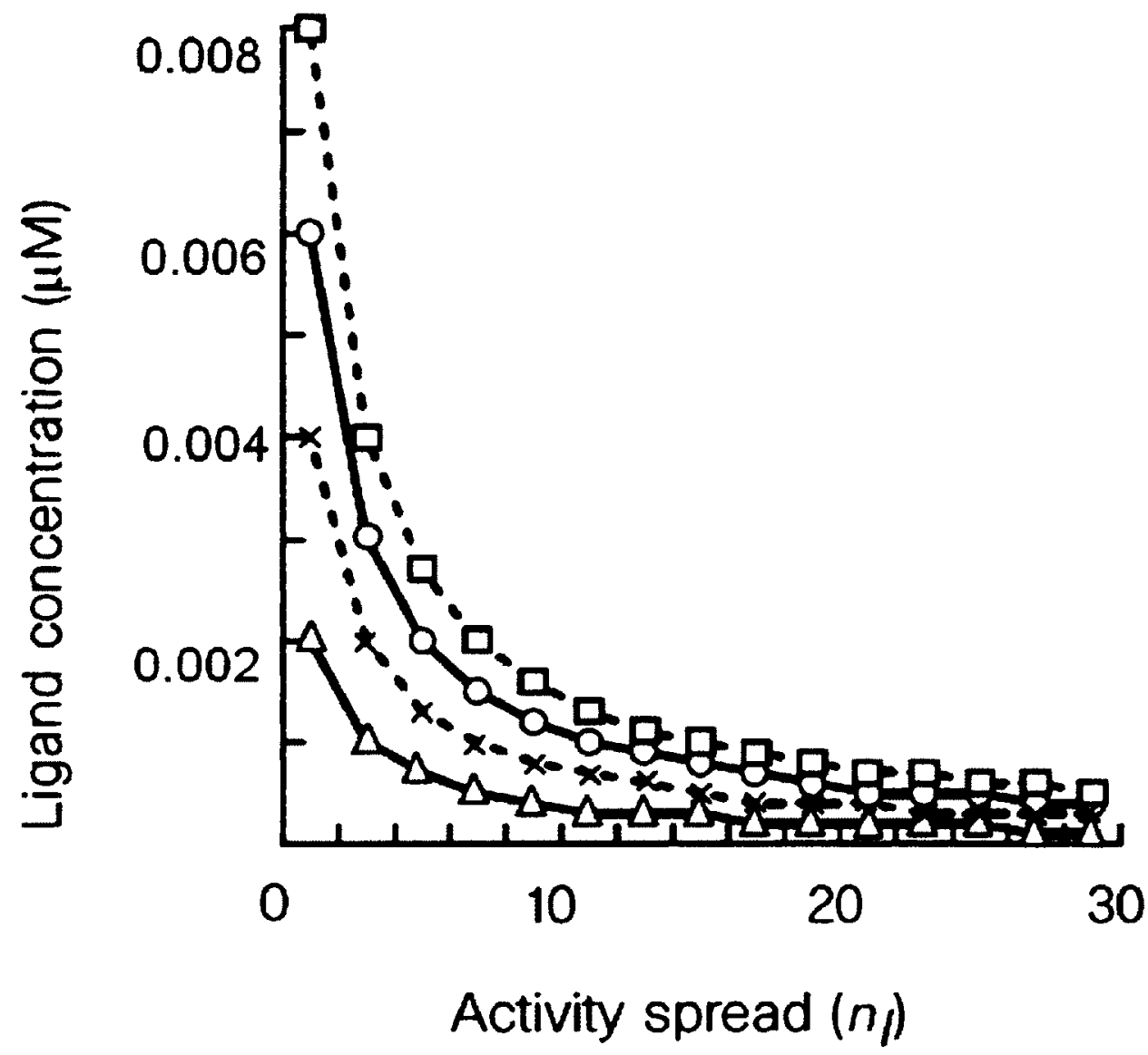

Figure 6.4: Theoretical effect of activity spread (higher probability of effecting neighboring receptor molecules) on detection of model ligand for one (triangles), two disjointed (crosses), three disjoined (circles), and four disjointed (squares) clusters ${ }^{230}$.

The probability of a receptor not affecting a neighboring receptor upon ligand binding is given by the ratio:

$$
\left(1-\frac{n_{1}}{N}\right)
$$


Thus upon binding, $n_{1}$ receptors will change their activity. A second ligand has $\mathrm{N}-1$ sites to bind to the cluster, so the probability of the second ligand to not affect the activity of a neighboring receptor $\left(n_{1}\right)$ is:

$$
\left(1-\frac{n_{1}}{N-1}\right)
$$

Since these two events are independent, the probability that either one will not affect another receptor is the product of the two:

$$
\left(1-\frac{n_{1}}{N}\right) \quad\left(1-\frac{n_{1}}{N-1}\right)
$$

Thus, for a single cluster interconnected, the probability a single ligand molecule effecting more than one receptor molecule is high (large $n_{1}$ ), while for a system with less interconnected receptor molecules, the probability of effecting a neighboring receptor is relatively lower ${ }^{230}$.

This theory can be extended to aptamer clusters as well. Various groups (already discussed) have found that DNA superstructures such as clusters (aggregates, micelles, nanostructures, etc) can lower the apparent $K_{d}$. We believe that this is due to receptor clustering, where the clustering increases the effective change seen in the activity $\left(n_{1}\right)$, and thereby, increases the signal detection. As a modification to what has already been 
shown in literature, we hypothesize that this theory can also be applied to aptamer superstructures of different sizes, where the larger aggregates will have larger change in $n_{1}$, and thereby, provide a better detection signal (lower apparent $K_{d}$ ). This would increase the applicability of aptamer-based superstructures for ultra-sensitive detection in biological systems such as drug delivery. In this project, we found that the nanoaggregates bound to the target (fluorophore) exhibited a size-dependant apparent $K_{d}$ with the larger assemblies having the best binding affinities. We hypothesize that when the aptamer binds to a target in an aptamer cluster, the ensuing shape change will affect neighboring aptamer strands (conformationally). Increasing the number of clusters allows for a more diffuse effect upon target binding (i.e., an increase in affected strands as cluster size is increased), thereby increasing sensitivity, similar to biological receptors-ligand systems discussed. In terms of detection, such as anisotropy used in this project, this size-related change will alter the rotational diffusion of the fluorophore depending on the number of aptamers affected. For example, the largest clusters will have the most number of aptamers affected and thus the best sensitivity as binding of few molecules can be measured anisotropically. Using other techniques such as QCM$D^{231}$ would give similar results as it would allow for the measurement of number of affected strands, extrapolated from the change in frequency decay curves at different cluster sizes. 


\subsection{Experimental}

\subsubsection{Materials}

Dodecanoic anhydride (98\%) was purchased from Sigma-Aldrich. All other reagents were purchased as already described in previous chapters and used without further purification. All glassware was rinsed $5 X$ with distilled and $5 X$ with deionized water)

\subsubsection{Methods}

Aptamer-alkyl conjugate: Amine functional sulforhodamine B aptamer (SA) was synthesized as described previously. After synthesis, the aptamer, bound to the controlled pore glass support columns, was placed in $1 \mathrm{~mL}$ of a saturated solution of lauric anhydride in anhydrous dimethylformamide (DMF). Prior to lauric anhydride addition, the DMF was purged with argon for 20 minutes to remove any moisture/air to limit premature hydrolysis of the anhydride. Aptamer and the anhydride were allowed to react for $\mathbf{4 8}$ hours at RT. Excess anhydride was removed by centrifugation and removal of the supernatant along with washing $2 \times$ with anhydrous acetonitrile. The $C_{12}$ modified aptamer conjugate (C12-SA) was cleaved off the CPG support by the addition of $1.5 \mathrm{~mL}$ of $48 \%$ ammonium hydroxide and heating of the solution at $32^{\circ} \mathrm{C}$ for 48 hours. The product was purified by PAGE using a $12 \%$ gel run for 1.5 hours at $45 \mathrm{~mA}$. The successful synthesis was confirmed by ESI-MS (calc'd M/Z C12SA: 9509; found: 9507.5).

Formation of $\mathrm{C}_{12}-\mathrm{SA}$ aggregates: $500 \mu \mathrm{L}$ of the alkyl-SA solution in deionized water was dried with argon on the bottom of a $5 \mathrm{~mL}$ glass vial yielding an aptamer film. It was 
necessary to desalt the alkyl-SA solution by (1) ethanol precipitation ( $2 \times)$; and (2) desalting tubes (Amicon Ultra $0.9 \mathrm{~mL} 3 \mathrm{~K}$ membranes) in order to achieve a uniform film on the glass surface. The solution should not be dried on a hot plate. The dried film was rehydrated in $500 \mu \mathrm{L}$ of deionized water under ultrasonication (Bransonic Ultrasonic cleaner $1210 \mathrm{R}-\mathrm{MT}$ ) at $80^{\circ} \mathrm{C}$ for 1.5 hours. The formation of aggregates was confirmed using scanning electron microscopy (SEM; Tescan Vegall XMU), dynamic light scattering (DLS, NanoDLS Brookhaven Instruments Corporation), and atomic force microscopy (AFM; Ntegra NT-MDT). The morphology of the particles was investigated using SEM by drying a drop of the micelle solution on a glass slide and imaging with and without a gold/palladium coating at RT. Analysis for DLS was performed at RT on varying concentrations of aggregates in water. The size distribution was confirmed by looking at the height profiles of the aggregates with AFM in air at $23.5{ }^{\circ} \mathrm{C}$ and relative humidity of $42.5 \%$ with $512 \times 512$ points per image. A $100 \times 100 \mu \mathrm{m}^{2}$ scanner (Ntegra) and cantilevers with rotated monolithic silicon probes $(125 \mu \mathrm{m}$-long, $40 \mathrm{~N} / \mathrm{m}$ spring constant Tap300Al, resonance frequency $300 \mathrm{kHz}$, Budget Sensors) were used. AFM samples were prepared by placing $50 \mu \mathrm{L}$ of freshly prepared extruded aggregates on a cleaved mica substrate and drying under a gentle stream of argon.

Extrusion of aggregates: After sonication, $100 \mu \mathrm{L}$ of the aggregate solution was extruded (Avanti mini-extruder) through filter sizes of 100, 80, and 30nm (Whatman Nuclepore Track-Etch Membrane). Samples were collected in two fractions: greater than $100 \mathrm{~nm}$ and less than $100 \mathrm{~nm}$. Extrusion was always done on freshly prepared aggregates (i.e., 
immediately after sonication) at room temperature to ensure maximum amount of aggregates.

Apparent Dissociation Constant Experiments: Apparent dissociation studies were performed for the unaggregated alkyl-SA conjugate and the alkyl-SA aggregate fractions. The dissociation studies were performed using fluorescence anisotropy (Horiba Jobin Yvon Fluorolog, model: FL-1039/40) of the target molecule dye, sulforhodamine B $(e x c / e m=556 \mathrm{~nm} / 582 \mathrm{~nm})$ as it bound to the aptamer. For all samples, the solutions were heated to $70^{\circ} \mathrm{C}$ for 10 minutes and cooled to RT prior to testing. $50 \mu \mathrm{L}$ of varying aggregate concentrations (made by serial dilution) were mixed with $50 \mu \mathrm{L}$ sulforhodamine $B\left(1.0 \times 10^{-6} \mathrm{M}\right)$ in $0.1 \mathrm{M} \mathrm{KCl}$. All measurements, in particular the most dilute aggregate samples, were tested immediately to avoid disassembly of the aggregates. Six data points were examined for each concentration and the averages were used for the $K_{d}$ calculation. For both experiments, the $K_{d}$ 's and lines of best fit were evaluated by minimizing the residuals values between calculated and observed experimental $\Delta$ anisotropy data using the solver feature of Microsoft Excel ${ }^{232}$.

\subsection{Results and Discussion}

\subsubsection{Synthesis of SA-alkyl conjugate}

We synthesized aptamer-alkyl aggregates to investigate the effects of receptor clustering on binding affinity. We used a $C_{12}$ tail, which has already been shown to form superstructures when conjugated with biological molecules ${ }^{233}$. As already discussed, a larger aggregate size (assuming it is interconnected) should increase detection 
sensitivity and lower the $K_{d}$. To make the aggregates, we decided to make our aptamer amphiphilic, thereby, driving aggregate formation by hydrophobic forces. In our case, due to the small hydrophobic tail, in comparison to the polar aptamer strand (high hydration affinity and low hydrophobic repulsion) we believe that we have formed aggregates of varying sizes. Our aptamer-alkyl conjugate is shown in Figure 6.5 below.

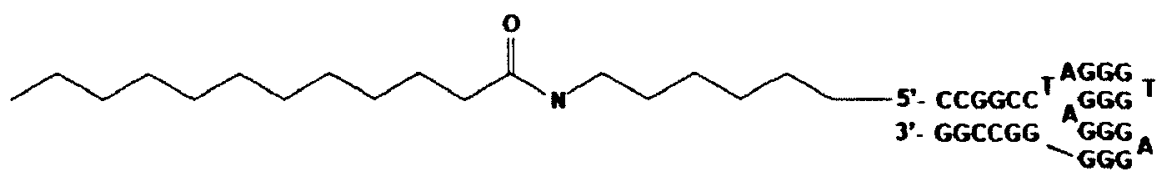

Figure 6.5: C12-SA conjugate used in this project showing the $C_{12}$ hydrophobic tail and polar DNA head.

The conjugate was purified by PAGE (Figure 6.6 (a)). The yield for the conjugate was low $(\sim 50 \%)$, however, the aim of this project was to find an easy and relatively fast way to form these conjugates. Other groups have used phosphoramidite chemistry on the DNA synthesizer to form such systems at high yields ${ }^{234}$, however, in many cases the modifier is not readily available and is prepared by a complicated multi-step reaction which can consume significant time and cost. 

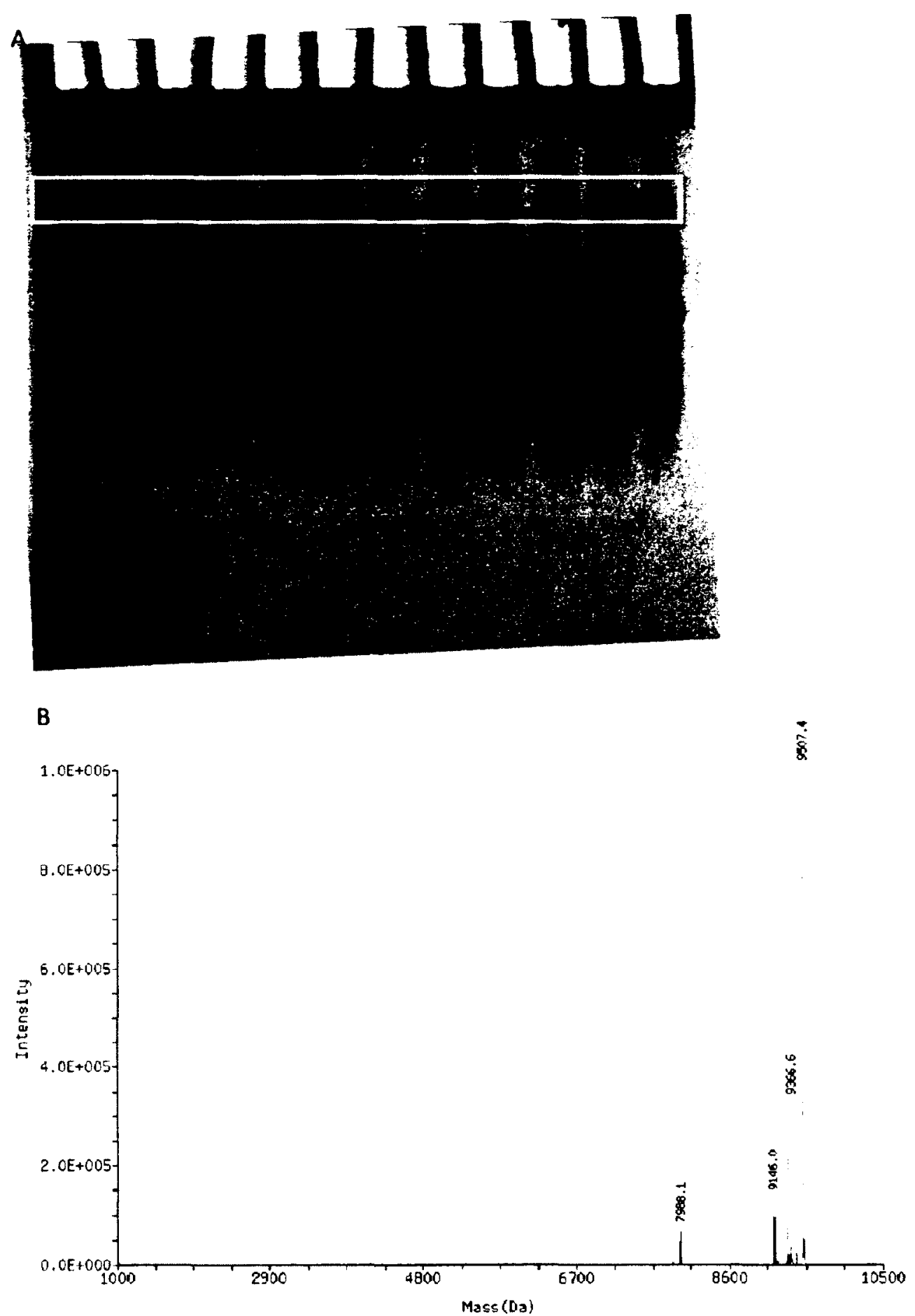

Figure 6.6: (a) 12\% PAGE (90min; 45mA) of crude $\mathrm{C}_{12}$-SA in all lanes showing the product in band 1 (white box); and (b) ESI-MS of $\mathrm{C}_{12}$-SA product (white box in (a)). 
Synthesis of the conjugate was confirmed by ESI-MS (calc'd M/Z C12SA: 9509; found: 9507.5; Figure 6.5(b)). The aggregates were formed by the well-established thin film hydration technique for liposomes ${ }^{235}$ (Figure 6.6). As suggested by our findings, we believe that the short tail was long enough to allow formation of nano-aggregates using thin film hydration.

\subsubsection{SA-alkyl nano assemblies/aggregates}

More recently, thin film hydration and slightly modified forms have been used to form superstructures of polymers ${ }^{236}$, casein micelles $^{237}$, solid lipid nanoparticles ${ }^{238}$ etc. In brief, a sample is dried on a flat surface, ensuring that all water has been removed. The sample is then rehydrated under sonication at temperatures $>50^{\circ} \mathrm{C}$. Due to sonication and high temperature, the molecules feel the entropic force of water molecules and are pushed together into superstructures to form thermodynamically stable states. By doping the aqueous solution, various groups have shown successful encapsulation of large molecules such as enzymes ${ }^{239}$, drugs ${ }^{240}$, oils ${ }^{241}$, etc. Our thin film hydration methodology is shown in Figure 6.7. 

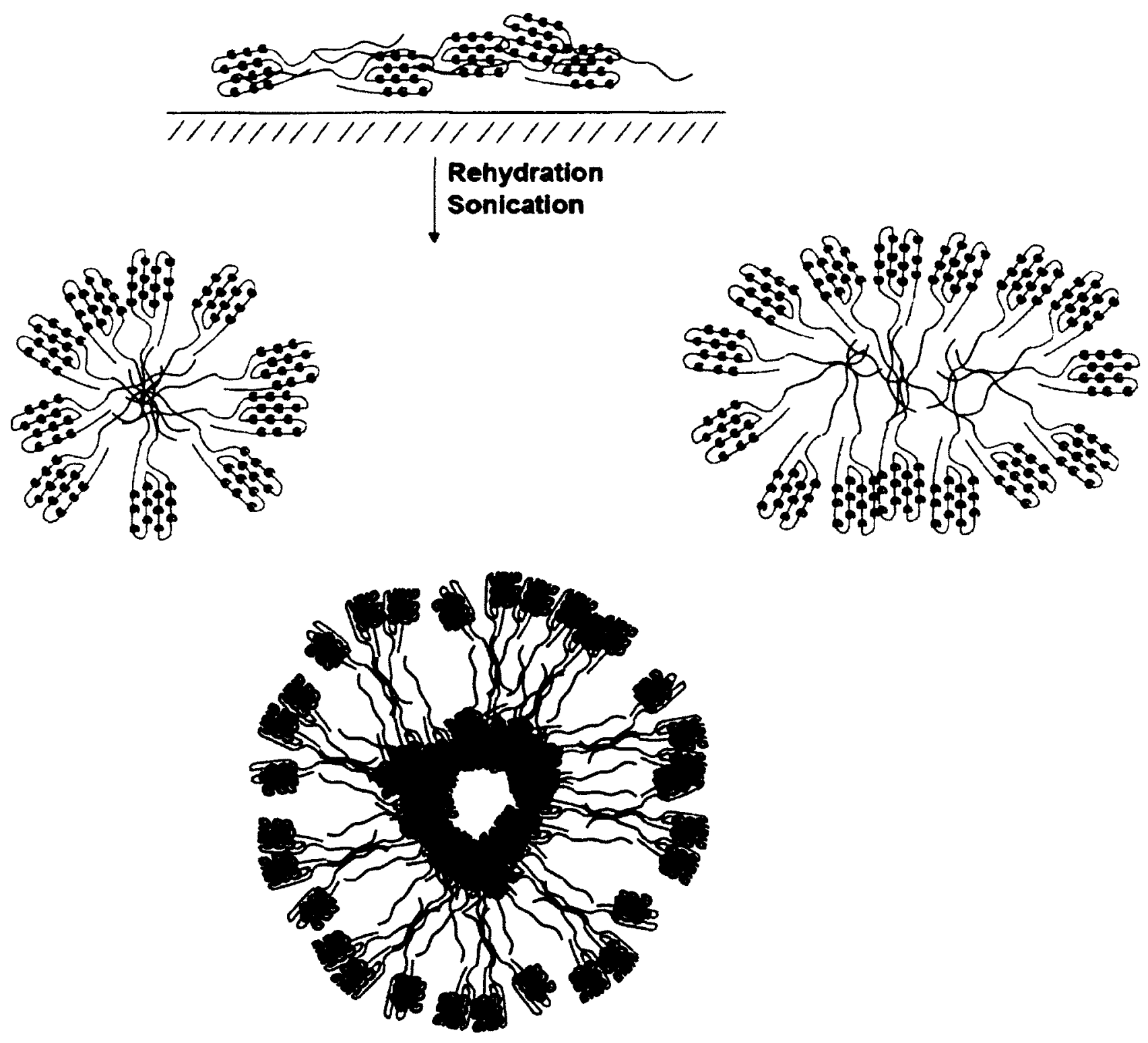

Figure 6.7: Strategy for aptamer aggregate preparation. Rehydration and sonication of an aptamer sample, after deposition onto a glass surface, yields aptamer aggregates of varying size and morphology.

As can be seen from our experimental strategy, a variety of morphologies might be present in our sample, which is why we refer to them as aggregates. Based on the 
theory of receptor clustering, the type of phase should have little effect on the $K_{d}$ 's. The process is driven mainly by the interconnected aggregate and the number of nearest neighbors which can be affected (size). The aggregates were characterized by SEM, AFM, and dynamic light scattering (DLS). The SEM of the aggregates is shown in Figure 6.8. The samples were coated with a gold/palladium film.

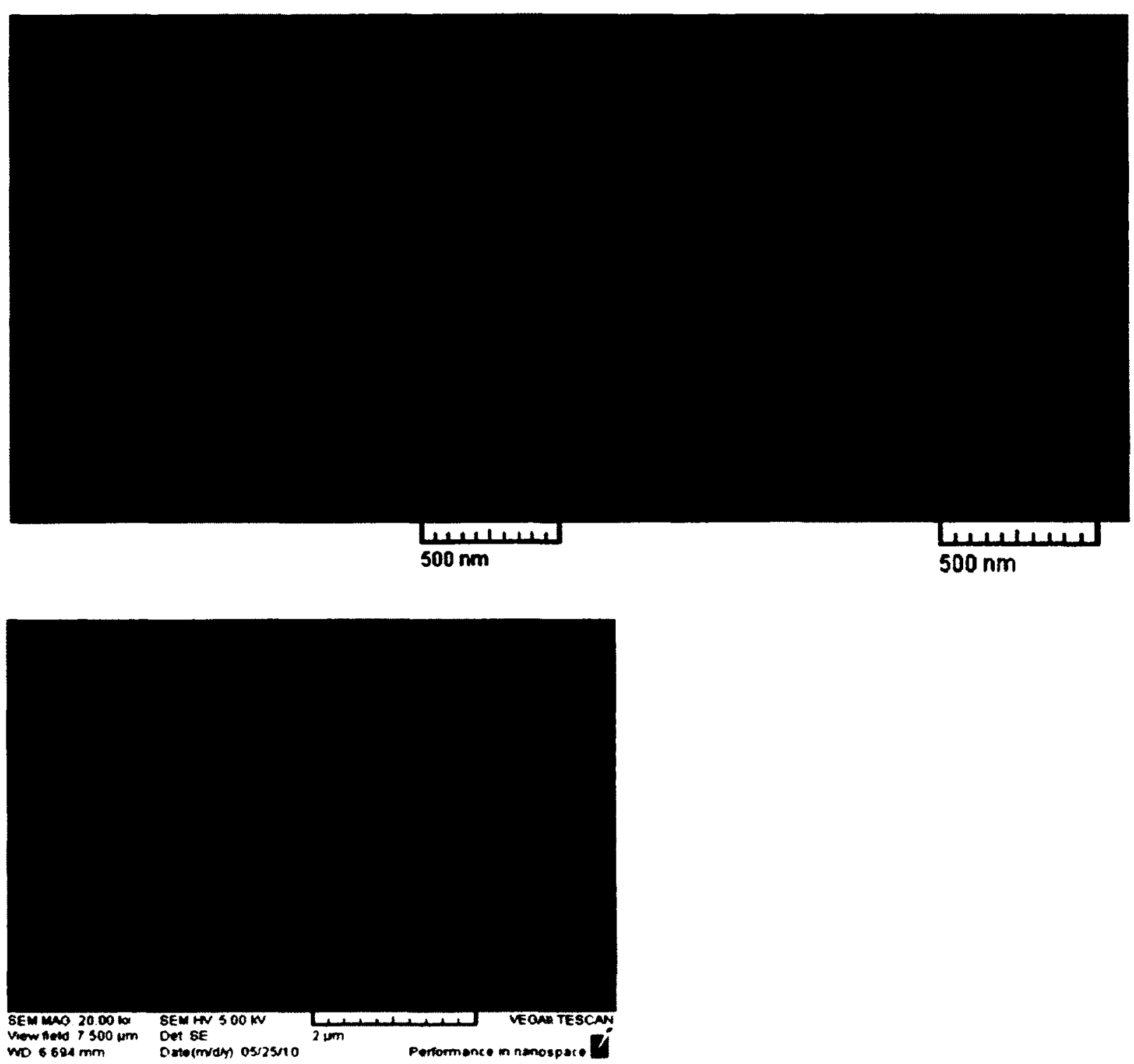

Figure 6.8: SEM of $C_{12}-S A$ aggregates formed. Sizes range $>100 \mathrm{~nm}$ with various shapes of aggregates. 
As can be seen from the SEM, the size of aggregates was predominantly $>100 \mathrm{~nm}$. Subsequent tests for $K_{d}$ evaluation and size fractionation suggest that there are aggregates $<100 \mathrm{~nm}$ present in our sample as well. A review by Barbarisi and coworkers $^{242}$ discusses in great detail the need to prepare biological samples appropriately for SEM imaging to remove artifacts, including morphology deformation caused by the result of dehydration (drying), and collapse of the biological sample (under vacuum). We hypothesize the lack of $<100 \mathrm{~nm}$ seen under SEM are a result of these artifacts, probably as a consequence of dehydration which causes the smaller aggregates to fall into smaller sub-units due to the removal of interstitial stress and effect of hydrophobehydrophobe repulsion. To detect the smaller units, we looked at AFM. In non-contact AFM, a tip is moved across a sample surface and deflections as a result of charge repulsion or attraction are measured. Sample imaging is typically done at RT with no vacuum required. As a result, soft (and relatively wet) samples can be imaged ${ }^{243}$. Indeed, using AFM on the unfractionated aggregates, we were able to see a large size distribution including particles $<100 \mathrm{~nm}$ (Figure 6.9). We also tried assessing particle size by DLS, however, since DNA molecules scatter light only weakly ${ }^{244}$, we were only able to get size distributions of concentrations $>1 \mu \mathrm{M}$ (data not shown). 

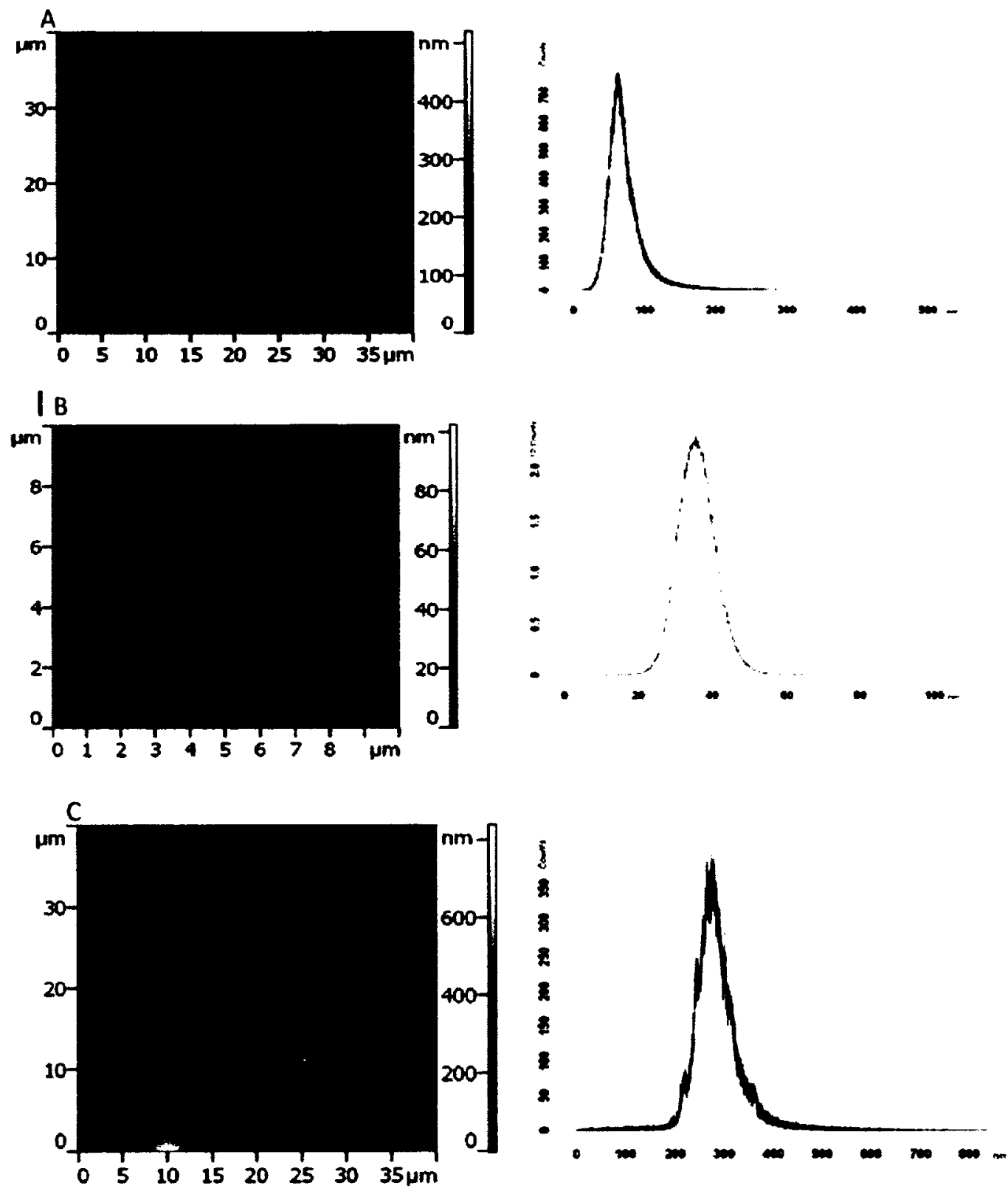

Figure 6.9: Characterization of the Aptamer Aggregates by AFM. AFM images (left) show that $\mathrm{C}_{12}$-SA aggregates in the size range of less than $100 \mathrm{~nm}$ are present (Distributions are shown on the right) ( $a$ and $b$ ). Larger aggregates, in the range of 100$300 \mathrm{~nm}$ were also seen (c). 


\subsubsection{Size selective apparent dissociation constants $\left(K_{d}\right)$}

Size-fractionation (extrusion) was done on freshly prepared aggregates. The set-up for the extruder is shown in Figure 6.10 (image taken from manufacturer of extruder used in laboratory). The extrusion allowed for fractionation of $<100 \mathrm{~nm}$ and $>100 \mathrm{~nm}$ fractions. The fractions were quantified using UV/vis, with the assumption that the extinction coefficient of the aggregates does not change relative to the free aptamer in solution $\left(C_{12}-S A\right)$, thus, following Beer-Lambert law.

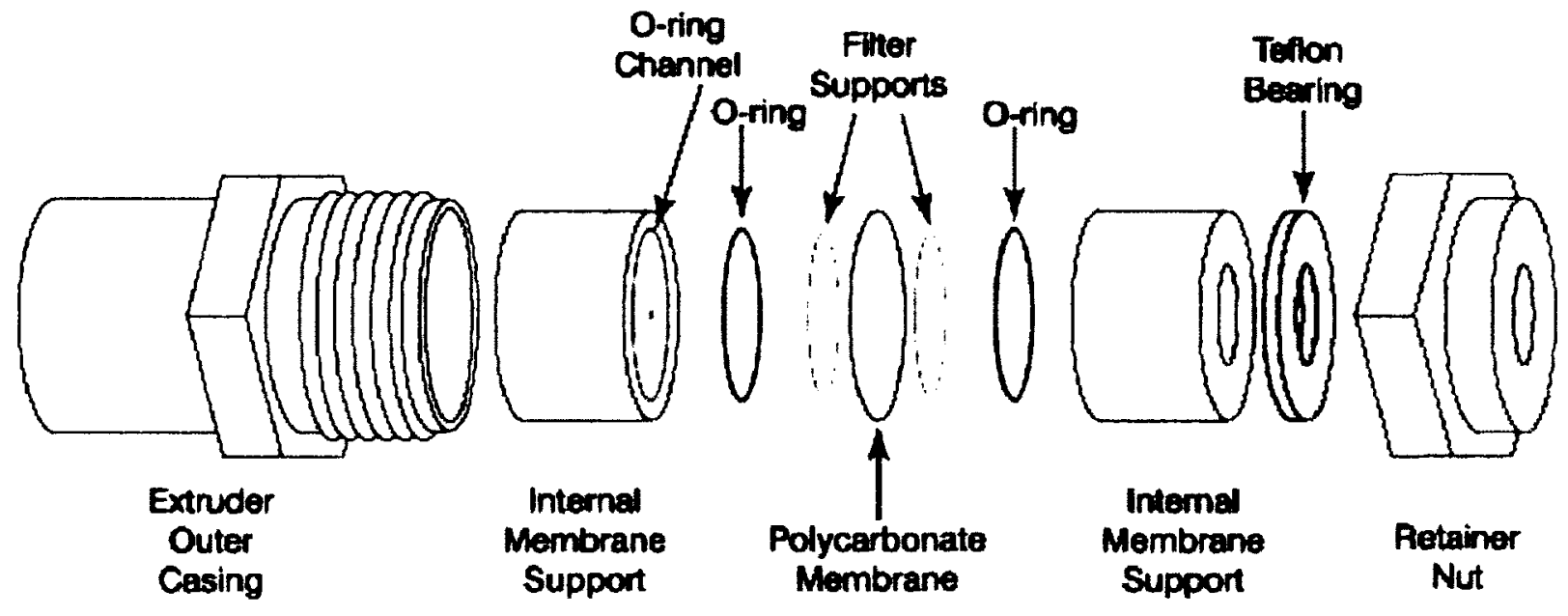

Figure 6.10: Extruder set-up used for size fractionation. ${ }^{245}$ Membranes of different sizes can be used to fractionate different sized particles. Membranes are based on size cutoffs.

Next, the fluorescence anisotropy of the sulforhodamine target was tested in the presence of various dilutions of the aggregates in order to determine the $K_{d}$. The results are shown in Figure 6.11. In solution, the unmodified sulforhodamine B aptamer has a $K_{d}$ of ca. $660 \mathrm{nM}^{246}$. Notably, conjugation of the aptamer with the long alkyl chain had only a small effect on the observed $\mathrm{K}_{d}$. Free $\mathrm{C}_{12}$-SA dissolved in water with $0.1 \mathrm{M} \mathrm{KCl}$, but 
not first aggregated via the thin film hydration method, was found to have an apparent dissociation constant on the order of $100 \mathrm{nM}$, slightly lower than the result for the unmodified sequence. Once in the aggregated form, however, the apparent $K_{d}$ of the polydisperse sample before extrusion improves by three orders of magnitude to ca. 300 pM. The variability in the size of the aggregates in our samples, as well as light scattering which can complicate fluorescence anisotropy measurements, is likely responsible for the large variation in the measured points. After extrusion through the $100 \mathrm{~nm}$ membrane, the two fractions (larger than and smaller than $100 \mathrm{~nm}$ in diameter) were also tested for apparent binding affinity. Interestingly, the smaller sized fraction had an intermediate $K_{d}$ of ca. $10 \mathrm{nM}$, while the larger sized aggregates had an average $K_{d}$ of $0.3 \mathrm{pM}$. The latter value represents a remarkable, six-order of magnitude improvement on the binding affinity of the unaggregated aptamer. If our apparent $K_{d}$ measurements were wholly a direct result of light scattering we should see a lower $K_{d}$ for the unextruded aggregates, that is, an additive effect should have been seen for the mixed fraction due to increased scatter. Thus, we believe that the increased binding affinity is not a result of scatter. This was also confirmed by running a negative control dye (tetramethylrosamine, TMR) with the largest sized fraction $(>100 \mathrm{~nm})$ to ensure that the lowest $K_{d}$ we calculated was not from scatter. As can be seen in Figure 6.12, we estimated an apparent $K_{d}$ of ca. $1 \times 10^{-7} \mathrm{M}$, which may be even higher since we did not test concentrations on the top plateau (at high concentrations) to see where the change in anisotropy levels off. This apparent $k_{d}$ is ca. six orders of magnitude higher than the $K_{d}$ for $>100 \mathrm{~nm}$ aggregates upon aptamer target binding $(0.3 \mathrm{pM})$ at equivelant DNA 
concentrations suggesting that the apparent $K_{d}$ is not a function of scatter. The reason for an estimated apparent $K_{d}$ for TMR is likely because the sulforhodamine B aptamer will bind weakly to rigid planar dyes such as $T M R^{247}$. It is likely that while this binding is not seen in solution since it is very weak, due to receptor clustering already described, there is a certain enhancement in binding that yields an apparent $K_{d}$ in the $\mu \mathrm{M}$ range. Once assembled, the aptamer-alkyl aggregates were stable in solution for approximately 1 day. Apparent dissociation constant experiments performed on samples more than a day old showed reversion to the unaggregated values (data not shown), indicative of the disassembly of the aggregates. 


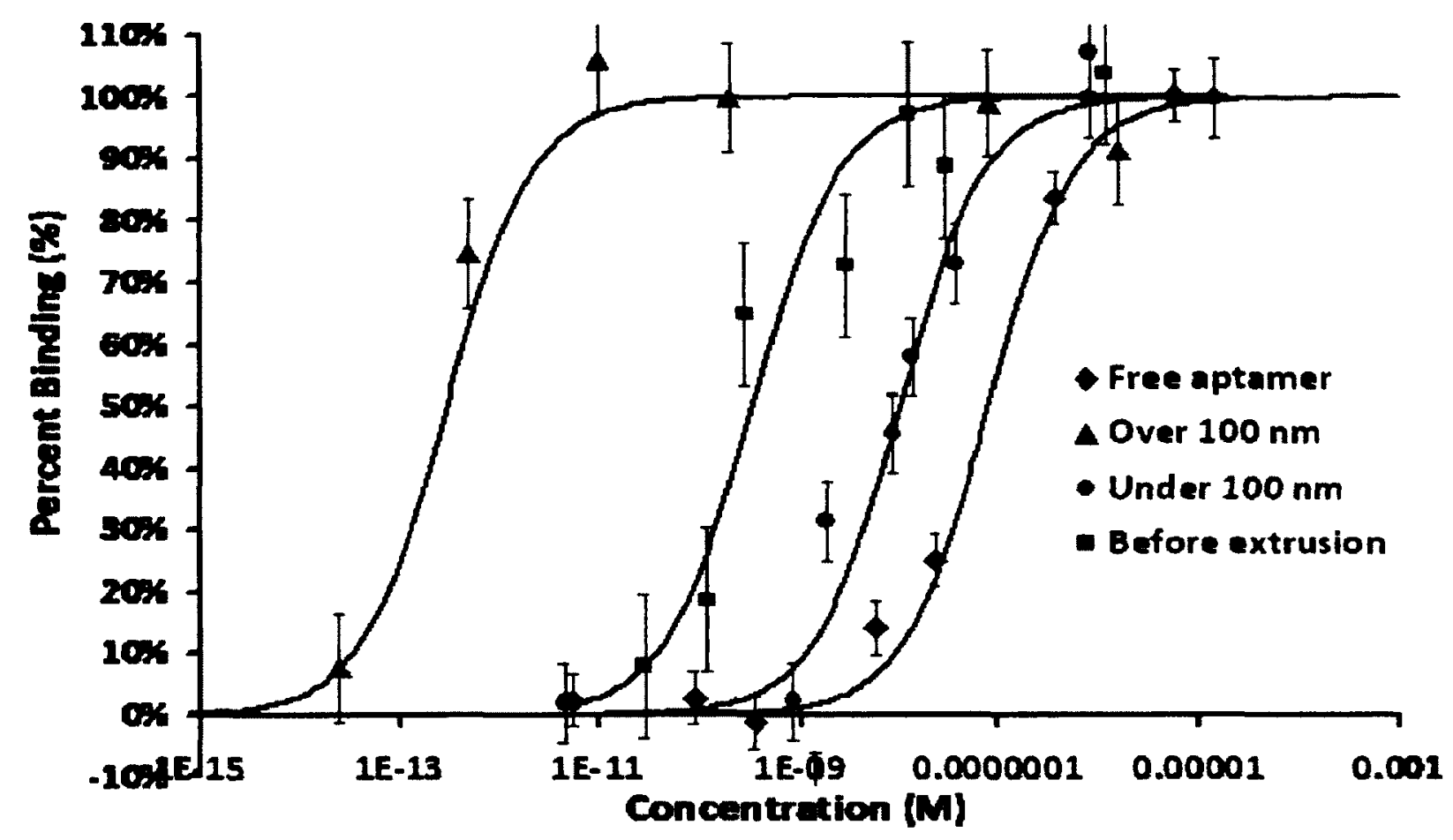

Figure 6.11: Apparent dissociation constant measurements before and after extrusion of the aggregate samples. Changes in the fluorescence anisotropy of Sulforhodamine B upon binding at different aptamer concentrations were used to determine the apparent $K_{d}$ values for $C_{12}-S A$ either in aggregated or free forms. Anisotropy data is shown as percent binding vs. free DNA concentration in order to better compare the different samples. The error on the concentration ranged from $\pm 1 \%$ for the first dilution to $\pm 3 \%$ for the most dilute serial concentration. For clarity, we did not measure aptamer concentrations down to $\mathrm{pM}$ range, rather we used our dye as a reporter molecule $(1 \mu \mathrm{M}$; emission intensities of $>10,000 \mathrm{cps}$ ) to measure changes in anisotropy upon binding with the differing concentrations of the nano-aggregates (as seen in graph). Lines of best fit were calculated as described in Experimental. Free alkyl aptamer (blue diamonds) has the highest apparent $K_{d}$ at $c a .100 \mathrm{nM}$. Before extrusion, the polydisperse sample (orange squares) has an apparent $K_{d}$ of ca. $300 \mathrm{pM}$. Extrusion to separate the aggregates into those smaller than $100 \mathrm{~nm}$ (red circles) and those larger than $100 \mathrm{~nm}$ (green triangles) reveals that the smaller sized fraction has an intermediate apparent $K_{d}$ of ca. $10 \mathrm{nM}$, while the larger sized aggregates have an average apparent $K_{d}$ of $0.3 \mathrm{pM}$. 




Figure 6.12: Anisotropy curve for $>100 \mathrm{~nm}$ aggregate fraction with negative control dye TMR. The apparent $K_{d}$ estimated here $\left(1 \times 10^{-7} \mathrm{M}\right.$, which may be even higher $)$ is six orders of magnitudes higher than that for the same fraction using the target dye, thereby confirming that scatter is not responsible for the binding affinity enhancement in the large fractions.

This dramatic enhancement in apparent binding affinity can be explained by the effect of receptor clustering. As already discussed, when a target molecule binds to an aptamer in an aggregate, the change in shape effects the shape of neighboring aptamers as well, thereby, increasing signal strength as the bound fluorophore is slowed down more than if it was bound to one aptamer strand. As we move from smaller to larger aggregates, the effect increases due to an increased number of aptamers in the aggregate and thus increase in the detectable signal. Our improvement in apparent binding affinity found for the aggregates in comparison to the free aptamers is larger than that recently reported in smaller aptamer-micelle samples $(\sim 3000 \times$ for the polydisperse sample, 6 orders of magnitude for our larger aggregates vs. $750 \times)^{248}$. This is 
likely the result of a greater number of clustered receptors densely packed in our larger structures, given the relationship between the number of receptors in an aggregate and the resulting binding affinity. As our approach yields aggregates in a range of sizes, this suggests that it might eventually allow for tuning of the aggregate sample to yield the desired apparent binding affinity, e.g. to select for the size of aggregates with the required apparent dissociation constant. Thus the novelty of this work lies not in the cooperative binding effect itself, which has been well-established elsewhere, but rather in the dramatic improvement of binding and the ease with which the aggregates can be prepared and tuned. The aggregates could be size-tuned, either physically by extrusion methods, or perhaps chemically with a better understanding of the effect of linker length on aggregate size.

\subsection{Conclusions}

In summary, we present herein a simple and general approach to the preparation of high-affinity aptamer aggregates from $C_{12}$ alkyl-modified aptamer conjugates. The strategy can be applied to aptamers for any target to yield particles with tailored molecular recognition properties. Current efforts include attempts to tune the size and shape of these aggregates and future work will examine the application of these assemblies to biosensing and controlled release.

\subsection{Acknowledgements}

Y.S thanks Prof. lanoul and Amy Wong from the lanoul lab for AFM imaging of the aggregates. 


\section{Chapter 7. Contributions to Knowledge and Future Studies}

From the research discussed herein, we were the first to demonstrate selectivity of an aptamer to its target when embedded within PEMs. We were also the first in this field to demonstrate controlled diffusion of the target and other small molecules in aptamer embedded polyelectrolyte microcapsules. By showing controlled diffusion using a possible candidate L-lysine, we have taken a step closer to the use of our smart fertilizer in in- situ systems. We have also demonstrated for the first time that aptamer apparent binding affinities can be size selectively tailored due to the principles of receptor clustering. From the findings demonstrated herein, we believe we have opened a new field for aptamer-based systems by showing possible use in harsh conditions, i.e., where the aptamer can be protected within a matrix but still be selective. This will allow for the use of these smart systems beyond just fertilizers, to areas of human health such as diagnostics and drug delivery.

Besides the obvious experiments which are still required before this system can be used for agriculture such as stability, matrix effects, functionality, etc there still remain future experiments before possible in situ use. First, polymers with better diffusion characteristics need to be evaluated. While polyelectrolytes are promising candidates, they have very high permeability and suffer high swelling effects. Low-permeability systems such as polymersomes (block copolymer bilayer vesicles) may allow for better control of diffusion. Second, optimization in terms of charge and number of bilayers will 
allow for selective tuning of the diffusion molecule through the films. Third, optimization of aptamer folding by tailoring the length of the sequence will allow for increased control of diffusion upon aptamer-target binding. Finally, more experiments are needed using electrochemistry to try and elucidate if along with charge selective diffusion, size of the permeating molecule can be used as a cut-off for diffusion. 


\section{Chapter 8. References}

${ }^{1}$ Interim Policy Statement on Health Canada's Working Definition for Nanomaterials. Access at: http://www.hc-sc.gc.ca/sr-sr/consult/_2010/nanomater/draft-ebaucheeng.php

${ }^{2}$ Auffan, M.; Rose, J.; Bottero, J.; Lowry, G.; Jolivet, J.; Wiesner, M. Nature Nanotechnology, 2009, 4, 634-641.

${ }^{3}$ Geng, D.; Lu, G. Jo. Nanopar. Res., 2007, 9(6), 1145-1151.

${ }^{4}$ Wise, F. Acc. Chem. Res., 2000, 33, 773-780.

${ }^{5}$ Roduner, E. Chem Soc. Rev., 2006, 35, 583-592.

${ }^{6}$ Eustis, S.; El-Sayed, M. Chem. Soc. Rev., 2006, 35, 209-217.

${ }^{7}$ Monreal, C.; McGill, W.; Nyborg, M. Can. Jo. Soil. Sci., 1986, 66, 499-511.

${ }^{8}$ Mikkelsen, R.; Shaviv, A. Fert. Res., 1993, 35, 1-12.

${ }^{9}$ Carpita, N.; Sabulase, D.; Montezinos, D.; Delmer, D. Science., 1979, 205, 144-147.

${ }^{10}$ Clarkson, D. "Root structure and sites of ion uptake." In Weisel, Y.; Eshel, A.; Kafkati, W. (eds) Plant roots, the hidden half. Marcel Dekker, Inc., New York, p331-373.

${ }^{11}$ Eichert, T.; Kurtz, A.; Steiner, U.; Goldbach, H. Pysiologia Plantarum, 2008, 134, $151-$ 160.

12 Zambryski, P. Jo. Cell Biol., 2004, 162, 165-168.

${ }^{13}$ Khodakovskaya, M.; Dervishi, E.; Mahmood, M.; Xu, Y.; Li, Z.; Watanabe, F.; Biris, A. ACS Nano, 2009, 3(10), 3221-3227.

${ }^{14}$ Lin, D.; Xing, B. Env. Sci. Tech., 2008, 42, 5580-5585.

${ }^{15}$ SciFinder Database ,https://scifinder.cas.org (accessed 14 March 2011).

${ }^{16}$ Hussein, M.; Zulkarnain, Z.; Yahaya, A.; Dickens, W. Jo. Cont. Rel., 2002, 82(2-3), $417-$ 427.

${ }^{17}$ Yavitz, E. US Patent 006014645 (2006).

${ }^{18}$ Hossain, K.; Monreal, C.; Sayari, A. Coll. Surf. B., 2008, 62, 42-50.

${ }^{19}$ Sultan, Y.; Walsh, R.; Monrael, C. DeRosa, M. Biomac., 2009, 10, 1149-1154.

${ }^{20}$ Yang, F. Liu, C.; Gao, F.; Su, M.; Wu, X.; Zheng, L.; Hong, F.; Yang, P. Biol. Trace Elem. Res., 2007, 119(1), 77-88.

${ }^{21}$ Lin, B. Jo. Forest Res., 2004, 15, 138-140.

22 Peter, N.; Long, S. Plant Physiol., 1988, 88, 396-400. 
${ }^{23}$ Bais, H.; Weir, T.; Perry, L.; Gilroy, S.; Vivanco, J. Annu. Rev. Plant. Biol., 2006, 57, 233266.

${ }^{24}$ Picart, C. Curr. Med. Chem., 2008, 15(7), 685-697.

${ }^{25}$ Hammond, P. Curr. Op. Coll. \& Interf. Sci., 2000, 4, 430-432.

${ }^{26}$ Decher, G.; Hong, J-D. Makromol Chem Macromol Symp., 1991, 46, 321-327.

27 Petty, M. Endeavour, 1983, 7(2), 65-69.

${ }^{28}$ Sastry, M.; Rao, M.; Ganesh, K. Acc. Chem. Res., 2002, 35(10), 847-855.

${ }^{29}$ Ariga, K.; Hill, J.; Ji, Q. Phys. Chem. Chem. Phys., 2007, 9, 2319-2340

${ }^{30}$ Zhang, W.; Zhang, A.; Guan, Y.; Zhang, Y.; Zhu, X. J. Mater. Chem., 2011, 21, 548-555.

${ }^{31}$ Becker, A.; Johnston, A.; Caruso, F. Macromol. BioSci., 2010, 10(5), 488-295.; Blacklock, J.; Hands, H.; Manickam, S.; Mao, G.; Mukhopadhyay, A.; Oupicky, D. Biomat., 2007, 28(1), 117-124.

${ }^{32}$ Suci, P.; Klem, M.; Arce, F.; Douglas, T.; Young, M. Langmuir, 2006, 22(21), 8891-8896.

${ }^{33}$ Ferreira, M.; Fiorito, P.; Oliveira, O.; Corboda de Torresi, S. Bios. Bioelec., 2004, 19(12), 1611-1615.

${ }^{34}$ List can be accessed at: http://www.sigmaaldrich.com/materials-science/materialscience-products.html?TablePage $=19812729$

35 Ariga, K.; Hill, J.; Ji, Q. Phys. Chem. Chem. Phys., 2007, 9, 2319-2340

${ }^{36}$ Schlenoff, J.; Ly, H.; Li, M. Jo. Am. Chem. Soc., 1998, 120(30), 7626-7634.

${ }^{37}$ Farhat, T.; Schlenoff, J. Langmuir, 2001, 17(4), 1184-1192.

${ }^{38}$ Schlenoff, J.; Ly, H.; Li, M. Jo. Am. Chem. Soc., 1998, 120(30), 7626-7634.

39 McAloney, R.; Sinyor, M.; Dudnik, V.; Goh, C. Langmuir, 2001, 17(21), 6655-6663.

${ }^{40}$ Mermut, O.; Barrett, C. J. Phys. Chem. B, 2003, 107, 2525-2530.

${ }^{41}$ Kohler, K.; Shchukin, D.; Mohwald, H.; Sukhorukov, G. J. Phys. Chem. B, 2005, 109, 18250-18259.

${ }^{42}$ Fleet, G.; Cohen Stuart, M.; Scheutjens, J.; Cosgrove, T.; Vincent, B. Polymers and Interfaces. Chapman and Hall: London, 1993.

${ }^{43}$ Schlenoff, J.; Dubas, S. Macromol., 2001, 34, 592-598.

${ }^{44}$ Schoeler, B.; Kumaraswamy, G.; Caruso, F. Macromol., 2002, 35, 889-897.

45 Tanchak, O.; Barrett, C. Chem. Mater., 2004, 16, 2734-2739.

${ }^{46}$ Farhat, T.; Schlenoff, J. Langmuir, 2001, 17, 1184-1192. ; Antipov, A.; Sukhorukov, G.; Mohwald, H. Langmuir, 2003, 19, 2444-2448. 
${ }^{47}$ Antipov, A.; Sukhorukov, G.; Donath, E.; Mohwald, H. J. Phys. Chem. B, 2001, 105, 2281-2284.

${ }^{48}$ McAloney, R.; Sinyor, M.; Dudnik, V.; Goh, C. Langmuir, 2001, 17(21), 6655-5533.

49 Jewell, C.; Zhang, J.; Fredin, N.; Lynn, D. Jo. Cont. Release, 2005, 106(1-2), 214-233.

${ }^{50}$ Robertson, D.; Joyce, G. Nature, 1990, 344, 467-468.

${ }^{51}$ Turek, C.; Gold, L. Science, 1990, 249, 505-510.

${ }^{52}$ Ellington, A.; Szostak, J. Nature, 1990, 346, 818-822.

${ }^{53}$ Keefe, A.; Pai, S.; Ellington, A. Nature Rev., 2010, 9, 537-550.

${ }^{54}$ Sussman, D.; Nix, J.; Wilson, C. Nature Struc. Biol., 2000, 7(1), 53-57.

${ }^{55}$ Nimjee, S.; Rusconi, C.; Sullenger, B. Annu. Rev. Med., 2005, 56, 55-83.

${ }^{56}$ Bunka, D.; Stockley, P. Nature Rev., 2006, 4, 588-596.

${ }^{57}$ Nimjee, S.; Rusconi, C.; Sullenger, B. Annu. Rev. Med., 2005, 56, 55-83.

58 Jenison, R.; Gill, S.; Pardi, A.; Polisky, B. Science,1994, 263, 1425-1429.

${ }^{59}$ Poncelet, S.; Limet, J.; Noel, J.; Kayaert, M.; Galanti, L.; Collet-Cassart, D. Jo. ImmunoAssay, 1990, 11, 77-88.

${ }^{60}$ Bunka, D.; Stockley, P. Nature Rev., 2006, 4, 588-596.

${ }^{61}$ Keefe, A.; Pai, S.; Ellington, A. Nature Rev., 2010, 9, 537-550.

${ }^{62}$ Dhar, S.; Gu, F.; Langer, R.; Farokhzad, O.; Lippard, S. Pro. Nat. Acad. Sci., 2008, 105(45), 7356-7361.

${ }^{63}$ Bunka, D.; Stockley, P. Nature Rev., 2006, 4, 588-596.

64 Stoltenburg, R.; Reinemann, C.; Strehlitz, B. Biomol. Eng. 2007, 24, 381-403.

${ }^{65}$ Berezovski, M.; Drabovich, A.; Krylova, S.; Musheev, M.; Okhonin, V.; Petrov, A.; Krylov, S. J. Am. Chem. Soc., 2005, 127, 3165-3171.

${ }^{66}$ Bunka, D.; Stockley, P. Nature Rev., 2006, 4, 588-596.

${ }^{67}$ Sultan, Y.; Walsh, R.; Monreal, C.; DeRosa, M. Biomacromolecules, 2009, 10, 1149 1154.

${ }^{68}$ Ellington, A. Curr. Biol., 1994, 4(5), 427-428.

${ }^{69}$ Navani, N.; Li, Y. Curr. Op. Chem. Biol., 2006, 10, 272-281.

${ }^{70}$ Stojanovic, M.; Prada, P.; Landry, D. Jo. Am. Chem. Soc., 2001, 123(21), 4928-4931.

${ }^{71}$ So, H.; Won, K.; Kim, Y.; Kim, B.; Ryu, B.; Na, P.; Kim, H.; Lee, J. Jo. Am. Chem. Soc., 2005, 127(34), 11906-11907.; Yi, X.; Lubin, A.; Heeger, A.; Plaxco, K. Angew. Chemie, 2005, 117(34), 5592-5595. 
${ }^{72}$ McCauley, T.; Hamaguchi, N.; Stanton, M. Anal. Biochem., 2003, 391(2), 244-250.

${ }^{73}$ Ohk, S.; Koo, O.; Sen, T.; Yamamoto, C.; Bhunia, A. Jo. App. Microbiol., 2010, 109(3), 808-817.

${ }^{74}$ Chen, S.; Huang, C.; Chang, H. Talanta, 2010, 81(1-2), 493-498.

75 Navani, N.; Li, Y. Curr. Op. Chem. Biol., 2006, 10, 272-281.

${ }^{76}$ Su, S.; Nutiu, R.; Filipe, C.; Li, Y.; Pelton, R. Langmuir, 2007, 23(3), 1300-1302.

77 Du, Y.; Chen, C.; Li, B.; Zhou, M.; Wang, E.; Dong, S. Biosens. Bioelec., 2010, 25(8), 1902-1907.

${ }^{78}$ Zang, X.; Li, S.; Jin, X.; Li, X. Biosen. Bioelec., 2011, in press.

${ }^{79}$ Yuan, Y.; Yuan, R.; Chai, Y.; Zhup, Y.; Liu, Z.; Mao, L.; Guan, S.; Qian, X. Analytica Chim. Acta, 2010, 668(2), 171-176.

${ }^{80}$ Willis, M.; Collins, B.; Zhang, T.; Green, L.; Sebesta, D.; Bell, C.; Ellogg, E.; Gill, S.; Magallanez, A.; Knauer, S.; Bendele, R.; Gill, P.; Janic, N. Bioconj. Chem., 1998, 9(5), 572-582.

${ }^{81}$ Motulsky, H.; Christopoulus, A. GraphPad Prism Fitting Models to Biological Data using Linear and Nonlinear Regression: A practical Guide to Curve Fitting. V 4.0

82 Rupcich, N.; Nutiu, R.; Li, Y.; Brennan, J. D. Anal. Chem. 2005, 77, 4300-4307.

${ }^{83}$ Su, S.; Nutiu, R.; Filipe, C.D.; Li, Y.; Pelton, R.. Langmuir 2007, 23, 1300-1302.

${ }^{84}$ Balamurugan, S.; Obubuafo, A.; Soper, S. A.; Spivak, D. A. Anal. Bioanal. Chem. 2008, 390, 1009- 1021.

${ }^{85}$ Wilson, C.; Szostak, J. W. Chem. Biol. 1998, 5, 609-617.

${ }^{86}$ Buttry, D.; Ward, M. Chem. Rev., 1992, 92, 1355-1379.

${ }^{87}$ Lu, C.; Lewis, O. J. Appl. Phys. 1972, 43, 4385.

${ }^{88}$ Kanazawa, K.; Gordon, J. J. Vac. Sci. Technol. 1985, 57, 1770.

${ }^{89}$ Richter, R.; Mukhopadhyay, A.; Brisson, A. Biophys. Jo., 2003, 85(5), 3035-3047.; Chen, Q.; Tang, W.; Wang, D.; Wu, X.; Liu, F. Biosen. Bioelec., 2010, 26(2), 575-579.; Muller, L.; Sinn, S.; Dreschel, H.; Ziegler, C.; Wendel, H.; Northoff, H.; Gehring, F. Anal. Chem., 2010, 82(2), 658-663.

${ }^{90}$ Kanazawa, K.; Choo, N. Jo. Sensors, 2009, doi. 10.1155/2009/824947

${ }^{91}$ QCM100 - Quartz Crystal Microbalance Theory and Calibration. Instruction manual received with the QCM200 used in the laboratory.

92 Kanazawa, K.; Choo, N. Jo. Sensors, 2009, doi. 10.1155/2009/824947

${ }^{93}$ Vestal, M. Chem. Rev., 2001, 101(2), 361-375. 
${ }^{94}$ Douglas, D. Mass Spec. Rev., 2009, 28(6), 937-960.

95 Douglas, D.; Frank, A.; Mao, D. Mass Spec. Rev., 2005, 24(1), 1-29.

${ }^{96}$ Fenn, J.; Mann, M.; Meng, C.; Wong, S.; Whitehouse, C. Science, 1989, 246(4926), 6471.

${ }^{97}$ Mann, M.; Talbo, G. Curr. Opin. Biotech., 1996, 71(1), 11-9.

${ }^{98}$ March, R.; Mass Spec. Rev., 2009, 28(6), 961-989.

${ }^{99}$ Wollnik, H. Mass. Spec. Rev., 1993, 12(2), 89-114.

${ }^{100}$ Pleul, D.; Simon, F. Polymer Surfaces and Interfaces. $1^{\text {st }}$ edition. Springer: Berlin, 2008.

${ }^{101}$ Belu, A.; Graham, D.; Castner, D. Biomat., 2003, 3635-3653.

102 Pleul, D.; Simon, F. Polymer Surfaces and Interfaces. $1^{\text {st }}$ edition. Springer: Berlin, 2008.

${ }^{103}$ Benninghoven, A. Angew. Chem. Int. Ed. Engl., 1994, 33, 1023-1043.

${ }^{104}$ Pleul, D.; Simon, F. Polymer Surfaces and Interfaces. $1^{\text {st }}$ edition. Springer: Berlin, 2008.

${ }^{105}$ Sodhi, R.; Analyst, 2004, 129, 483-487.

${ }^{106}$ Colliver, T.; Brummel, C.; Pacholski, M.; Swanek, F.; Ewing, G.; Winograd, N. Anal. Chem. 1997, 69(13), 2225-22231.; Ishihara, M.; Ebata, S.; Kumondai, K.; Mibuka, R.; Uchino, K.; Yurimoto, H. Sur. Inter. Anal., 2010, 42(10-11), 1598-1602.; Debois, D.; Bralet, M.; LeNaour, F.; Brunelle, A.; Laprevote, O. Anal. Chem., 2009, 81(8), 28232831.; Chen, L.; Shah, S.; Silangcruz, J.; Eller, M..; Verkhoturov, S.; Rezvin, A.; Schweikert, E. Int. Jo. Mas. Spec.,2011, in press.; Shon, H.; Park, J.; Choi, I.; Park, H.; Moon, D.; Lee, T. Jo. Nanosci. Nanotech., 2011, 11(1), 638-641.

107 Sodhi, R.; Analyst, 2004, 129, 483-487.

${ }^{108}$ Rugar, D.; Hansma, P. Phys. Today, 1990, 23-30.

${ }^{109}$ Meyer, E. Prog. Surf. Sci., 1992, 41, 3-49.

${ }^{110}$ Murphy, D. Fundamentals of Light Microscopy and Electronic Imaging. John Wiley \& Sons: Canada, 2001.

${ }^{111}$ Vigoureux, J.; Courjon, D. App. Optics, 1992, 31(6), 3170-3177.

${ }^{112}$ Tamburini, F.; Anzolin, G.; Umbriaco, G.; Bianchini, A.; Barbieri, C. Phys. Rev. Lett., 2006, 97, 163903.

113 Pawley, J. Handbook of Biological Confocal Microscopy. $1^{\text {st }}$ edition. Plenumm Press: New York, 1990. 
${ }^{114}$ Baschong, W.; Suetterlin, R.; Laeng, R. Jo. Histochem. Cytochem., 2001, 49(12), $1565-$ 1571.; Ding, P.; Matzer, A.; Wolff, D.; Mente, J.; Pioch, T.; Staehle, H. Dental Mat., 2010, 26(3), 257-263.; Schopf, W.; Kudryavtsev, A.; Tripathi, A.; Czaja, A. Taphonomy, 2011, 32, 457-486.; Bridier, A.; Brissonnet, F.; Boubetra, A.; Thomas, V.; Briandet, R. Jo. Micro. Meth., 2010, 82(1), 64-70.

${ }^{115}$ Beaucage, S.; lyer, R. Tetrahedron, 1992, 48(12), 2223-2311.

${ }^{116}$ The difference in concentrations of the SA, RO, and CT DNA are not expected to lead to large differences in the amount of DNA deposited in the film due to charge compensation effects. See Rieglar, H; Essler, F. Langmuir 2002, 18, 6694-6698.

${ }^{117}$ Fylstra, D. H.; Lasdon, L.; Watson, J.; Waren, A. Interfaces 1998, 28, 29-55.; Nenov, I.P.; Fylstra, D.H. Reliab. Comput. 2003, 9, 143-159.

${ }^{118}$ Wilson, C.; Szostak, J. W. Chem. Biol. 1998, 5, 609-617.

${ }^{119}$ The films were: PDDA/PSS: (PDDA $)_{5}(\mathrm{PSS})_{5}, \mathrm{PDDA} / \mathrm{PSS} / \mathrm{SA}:(\mathrm{PDDA})_{11}(\mathrm{SA})_{5}(\mathrm{PSS})_{6}$, PDDA/PSS/RO: (PDDA) $)_{11}(\text { RO })_{5}(\mathrm{PSS})_{6}, \mathrm{PDDA} / \mathrm{PSS} / \mathrm{CT}$ : (PDDA) ${ }_{11}(\mathrm{CT})_{5}(\mathrm{PSS})_{6}$. For the oligo films, the deposition method was PDDA/PSS $\times 5$, PDDA/Oligo $\times 5$, PDDA/PSS $\times 1$, thus embedding the DNA in the polyelectrolyte film.

${ }^{120}$ McAloney, R.; Sinyor, M.; Dudnik, V.; Goh, C. Langmuir, 2001, 17, 6655-6663.

121 a) Antipov, A. A.; Sukhorukov, G. B.; Donath, E.; Möhwald, H. J. Phys. Chem. B, 2001, 105, 2281-2284. b) Yamauchi, F.; Koyamatsu, Y.; Kato, K.; Iwata, H. J. Biomaterials 2006, 27, 3497-3504. c) El Haitami, A. E.; Martel, D.; Ball, V.; Nguyen, H. C.; Gonthier, E.; Labbe, P.; Voegel, J.-C.; Schaaf, P.; Senger, B.; Boulmedais, F. Langmuir 2009, 25, 2282-2289.

${ }^{122}$ The films were: PDDA/PSS: (PDDA $)_{5}(\mathrm{PSS})_{5}, \mathrm{PDDA} / \mathrm{PSS} / \mathrm{SA}:(\mathrm{PDDA})_{11}(\mathrm{SA})_{5}(\mathrm{PSS})_{6}$, PDDA/PSS/RO: (PDDA) ${ }_{11}(\text { RO })_{5}(\mathrm{PSS})_{6}, \mathrm{PDDA} / \mathrm{PSS} / \mathrm{CT}:(\mathrm{PDDA})_{11}(\mathrm{CT})_{5}(\mathrm{PSS})_{6}$

${ }^{123}$ McAloney, R. A.; Sinyor, M.; Dudnik, V.; Goh, M. C. Langmuir 2001, 17, 6655-6663.

${ }^{124}$ Wilson, C.; Szostak, J. W. Chem. Biol. 1998, 5, 609-617.

${ }^{125}$ Although the nature of the features in the PDDA/PSS/CT films is unclear, they may be a result of deformation of the upper polymer layers due to the morphology of the CT DNA in the lower layers. Another possibility is that the CT film, after deposition, is more highly swollen than the other films (perhaps due to the size or charge on the CT DNA) and experiences rapid shrinking during the drying process that leads to this roughness. See Podsiadlo, P.; Michel, M.; Lee, J.; Verploegen, E.; Kam, N. W. S.; Ball, V.; Lee, J.; Qi, Y.; Hart, A. J.; Hammond, P. T.; Kotov, N. A. Nano Lett. 2008, 8, $1762-$ 1770.

${ }^{126}$ Wilson, C.; Szostak, J. W. Chem. Biol. 1998, 5, 609-617.

${ }^{127}$ Cooper, M. A.; Singleton, V. T. J Mol Recognit. 2007, 20, 154-184. 
${ }^{128}$ Johnston, A. P. R.; Caruso, F. J. Am .Chem. Soc. 2005, 127, 10014-10015.

${ }^{129}$ B. G. De Geest, A. M. Jonas, J. Demeester and S. C. De Smedt, Langmuir, 2006, 22, 5070.; H. Inoue, K. Sato, J. Anzai, Biomacromolecules, 2005, 6, 27.; B. G. De Geest, C. Dejugnat, G. B. Sukhorukov, K. Braeckmans, S. C. De Smedt, J. Demeester, Adv. Mater. 2005, 17, 2357.; D. T. Haynie, N. Palath, Y. Liu, B. Y. Li, N. Pargaonkar, Langmuir, 2005, 21, 1136.; B. G. De Geest, A. G. Skirtach, A. A. Mamedov, A. A. Antipov, N. A.Kotov, S. C. De Smedt, G. B. Sukhorukov, Small, 2007, 3, 804.; Z. H. Lu, M. D. Prouty, Z. H. Guo, V. O. Golub, C. Kumar, Y. M. Lvov, Langmuir, 2005, 21, 2042.; K. Katagiri, K. Koumoto, S. Iseya, M. Sakai, A. Matsuda, F. Caruso, Chem. Mater., 2009 21, 195.; S. Erokhina, L. Benassi, P. Bianchini, A. Diaspro, V. Erokhin, M. P. Fontana, J. Am. Chem. Soc. 2009, 131, 9800.; A. G. Skirtach, A. A. Antipov, D. G. Shchukin, G. B. Sukhorukov, Langmuir, 2004, 20, 6988.

${ }^{130}$ K. Glinel, G. B. Sukhorukov, H. Möhwald, V. Khrenov, K. Tauer, Macromol. Chem. Phys. 2003, 204, 1784. ;G. Ibarz, L. Dähne, E. Donath, H. Möhwald, Chem. Mater. 2002, 14, 4059. ; A. A. Antipov, G. B. Sukhorukov, H. Möhwald, Langmuir 2003, 19, 2444.; R. Georgieva, R. Dimova, G. Sukhorukov, G. Ibarz , H. Möhwald, J. Mater. Chem., 2005, 15, 4301.; G. B. Sukhorukov, A. A. Antipov, A. Voigt, E. Donath, H. Möhwald, Macromol. Rapid Commun. 2001, 22, 44.; Y. Lvov, A. Antipov, A. Mamedov, H. Möhwald, G. B. Sukhorukov, Nano Lett. 2001, 1, 125.; K. Glinel, G.B. Sukhorukov, H. Möhwald, V. Khrenov, K. Tauer, Macromol. Chem. Phys. 2003, 204, 1784.; A.A. Antipov, G.B. Sukhorukov, E. Donath, H. Möhwald, J. Phys.Chem., B, 2001 105, 2281.

${ }^{131}$ A. G. Skirtach, P. Karageorgiev, M. F. Bédard, G. B. Sukhorukov, H. Möhwald J. Am. Chem. Soc., 2008, 130, 11572.

${ }^{132}$ S. F. M. van Dongen, H.-P. M. de Hoog, R. J. R. W. Peters, M. Nallani, R. J. M. Nolte, J. C. M. van Hest, Chem. Rev. 2009, 109, 6212.; M. C. DeRosa, C. Monreal, M. Schnitzer, R. Walsh, Y. Sultan Nature Nanotech. 2010, 5, 91.

${ }^{133}$ Pawley, J. Handbook of Biological Confocal Microscopy. $1^{\text {st }}$ edition. Plenumm Press: New York, 1990.

${ }^{134}$ H.-G. Kapitza and E. Sackmann Biochim. Biophys. Acta, 1980, 595, 56.

135 Song, W.; He, Q.; Mohwald, H.; Yang, Y.; Li, J. Jo. Cont. Rel., 2009, 139, 160-166.; Liu, X.; Gao, C.; Shen, J.; Mohwald, H. Macromol. Biosci., 2005, 5, 1209-1219.; Johnston, A.; Such, G.; Caruso, F. Angew. Chem. Int. Ed.,2010, 49, 2664-2666.

${ }^{136}$ Borodina, T.; Markvicheva, E.; Kunizhev, S.; Mohwald, H.; Sukhorukov, G.; Kreft, O. Macromol. Rapid Comm., 2007, 28, 1894-1899.; Becker, A.; Johnston, A.; Caruso, F. Macromol. Biosci., 2010, 488-495.

${ }^{137}$ Dong, W.; Ferri, J.; Adalsteinsson, T.; Schonhoff, M.; Sukhorukov, G.; Mohwald, H. Chem Materi., 2005, 17, 2603-2611. 
${ }^{138}$ Beaucage, S.; lyer, R. Tetrahedron, 1992, 48(12), 2223-2311.

${ }^{139}$ Voigt, A.; Lichtenfeld, H.; Sukhorukov, G.; Zastrow, H.; Donath, E.; Baumler, H.; Mohwald, H. Ind. Eng. Chem. Res., 1999, 38, 4037.

${ }^{140}$ Shchukin, D.; Patel, A.; Sukhorukov, G.; Lvov, Y. Jo. Am. Chem. Soc., 2004, 126(11), 3374-3375.; Jewell, C.; Zhang, J.; Fredin, N.; Lynn, D. Jo. Con. Rel., 2005, 106(1-2), 214-223.; Zelikin, A.; Becker, A.; Johnston, A.; Wark, K.; Turatti, F.; Caruso, F. ACS Nano, 2007, 1(1), 63-69.

${ }^{141}$ Yu, A.; Wang, Y.; Barlow, E.; Caruso, F. Adv. Mat., 2005, 17, 1737-1741.; Kohler, K.; Mohwald, H.; Sukhorukov, G. J. Phys. Chem. B., 2006, 110, 24002-24010.

142 Sun, G.; Zhang, Z. Int. Jo. Pharm., 2002, 242(1-2), 307-311.

${ }^{143}$ Petrov, A.; Volodkin, D.; Sukhorukov, G. Biotech. Progress., 2005, 3, 918-925.; De Geest, B.; Vandenbroucke, R.; Guenther, A.; Sukhorukov, G.; Hennink, W.; Sanders, N.; Demeester, J.; Smedt, S. Avd. Mat., 2006, 18(8), 1005-1009.; Zhao, Q.; Zhang, S.;; De Koker, S.; De Geest, B.; Cuvelier, C.; Ferdinande, L.; Deckers, W.; Hennink, W.; De Smedt, S.; Mertens, N. Adv. Fun. Mat., 2007, 17(18), 3754-3763.; McShane, M. Methods Mol. Bio., 2011, 679, 147-154. Zhang, J.; Li, C.; Xue, Z.; Cheng, H.; Huang, F.; Zhuo, R.; Zhang, X. Acta Bio., 2011, in press.

144 Jin, Y.; Liu, W.; Wang, J.; Fang, J.; Gao, H. Coll. Surf. A., 2009, 342(1-3), 40-45.

${ }^{145}$ Halozan, D.; Riebentanz, U.; Brumen, M.; Donath, E. Coll. Surf. A, 2009, 342(1-3), 115121.

${ }^{146}$ Fujii, A.; Maruyama, T.; Ohmukai, Y.; Kamio, E.; Sotani, T.; Matsuyama, H. Coll. Surf. A. 2010, 356(1-3), 126-133.

${ }^{147}$ Ketrane, R.; Leleyter, L.; Baraud, F.; Jeanin, M.; Gil, O.; Saidani, B. Desalination, 2010, 262(1-3), 21-30.; Cheng, B.; Lei, M.; Yu, J.; Zhao, X. Mat. Lett., 2004, 58(10), 15651570.

${ }^{148}$ Zhang, L.; Shi, J.; Jiang, Z.; Jiang, Y.; Meng, R.; Zhu, Y.; Liang, Y.; Zheng, Y. ACS App. Mat. \& Int., 2001, in press.; Raamsdonk, J.; Chang, P. Jo. Biomed. Mat. Res., 2001, 54(2), 264-271.; Fery, A.; Dubreuil, F.; Mohwald, H. New Jo. Phys., 2004, 6, 18.

149 Peyratout, C.; Dahne, L. Angew. Chem. Int. Ed.., 2004, 43, 3762-3783.

${ }^{150}$ Tong, W.; Gao, C.; Shen, J. Eur. Pol. Journal., 2006, 42(12), 3341-3351.

151 Tong, W.; Dong, W.; Gao, C.; Mohwald, H. J. Phys. Chem. B., 2005, 109, 13159.; Tong, W.; Song, H.; Gao, C.; Mohwald, H. J. Phys. Chem. B., 2006, 110, 12905.

152 Froh, J.; Kohler, R.; Mohwald, H.; Krastev, R. Langmuir, 2010, 26(19), 15516-15522.

${ }^{153}$ Volodkin, D.; Petrov, A.; Prevot, M.; Sukorukov, G. Langmuir, 2004, 20, 3398-3406.; Ibarz, G.; Dahne, L.; Donath, E.; Mohwald, H. Chem. Mater., 2002, 14, 4059-4062.; Wang, F.; Feng, J.; Tong, W.; Gao, C. Jo. Mater. Chem., 2007, 17, 670-676.; Daiguji, H.; 
Matsuoka, E.; Muto, S. Soft Matter,2010, 6, 1892-2897.; Cornejo, J.; Matsuoka, E.;

Daiguji, H. Soft Matter, 2011, in press.

${ }^{154}$ Manders, E.; Verbeek, F.; Aten, J. Jo. Microscopy, 1993, 169, 375-382.; Wouterlood, F.; Denderen, J.; Blijleven, N.; Minnen, J.; Hartig, W. Brain Res. Prot., 1998, 2(2), 149 159.; Konietzko, U.; Goodger, Z.; Meyer, M.; Kohli, B.; Bosset, J.; Lahin, D.; Nitsch, R. Neuro. Aging, 2010, 31(1), 58-73.; Zhou, R.; Yazdi, A.; Menu, P.; Tschopp, J. Nature, 2011, 469, 221-225.

155 Antipov, A.; Sukhorukov, G.; Mohwald, H. Langmuir, 2003, 19, 2444.

${ }^{156}$ Ibarz, G.; Dahne, L.; Donath, E.; Mohwald, H. Chem. Mater., 2002, 14, 4059.

${ }^{157}$ Han, S.; Lindholm-Sethson, B. Electrochim. Acta, 1999, 45, 845.

${ }^{158}$ Brett, C.; Brett, A. Electrochemistry Principles, Methods, and Applications. Oxford University Press: USA, 1993.

${ }^{159}$ Davies, C. Electrochemistry. William Clowes and Sons: London, 1967.

${ }^{160}$ Rismani-Yazdi, H.; Carver, S.; Christy, A.; Tuovinen, O. Jo. Power Sources, 2008, 180, 683-694.

${ }^{161}$ Wang, J. Analytical Electrochemistry. John Wiley \& Sons: USA, 2006.

162 Cottrell, F.; Z Physik. Chem., 1902, 42, 385.

${ }^{163}$ Raj, C.; Ohsaka, T. Jo Electroanalytical Chemistry, 2003, 540(2), 69-77.

${ }^{164}$ Brett, C.; Brett, A. Electrochemistry Principles, Methods, and Applications. Oxford University Press: USA, 1993.

${ }^{165}$ Adapted from BioLogic Science Instruments: http://www.biologic.info/potentiostat/glassware_cells.html. Accessed January $29^{\text {th }} 2011$.

${ }^{166}$ Kissinger, P.; Heinemann, W. J. Chem. Ed. 1983, 60, 702.

${ }^{167}$ Brett, C.; Brett, A. Electrochemistry Principles, Methods, and Applications. Oxford University Press: USA, 1993.

${ }^{168}$ Haberska, K.; Ruzgas, T. Bioelectrochem., 2009, 76(1-2), 153-161.

${ }^{169}$ Harris, J.; DeRose, P.; Bruening, M. Jo. Am. Chem. Soc., 1999, 121(9), 1978-1979.

${ }^{170}$ Vered, P.; Eugenii, K.; Lioubashevski, O.; Willner, I. Langmuir, 2001, 17(4), 1110-1118.

${ }^{171}$ Harris, J.; Bruening, M. Langmuir, 2000, 16(4), 2006-2013.

${ }^{172}$ Fulghum, T.; Estillore, C.; Steven, V.; Armes, P.; Advincula, R. Macromolecules, 2008, 41(2), 429-435.

${ }^{173}$ Ammam, M.; Keita, B.; Nadjo, L.; Mbomekalle, I.; Fransaer, J. Jo. ElectroAnal. Chem., 2010, 645(2), 65-73. 
${ }^{174}$ Liu, X.; Wang, F.; Han, S.; Shi, L.; Xu, G., Electroanalysis, 2010, 22(9), 963-968.

${ }^{175}$ Munge, B.; Dowd, R.; Krause, C.; Milord, L. Electroanalysis, 2010, 21(20), 2241-2248.

${ }^{176}$ Pays, M.; Korba, T.; Bos, M.; van der Linden, W. Talanta, 1991, 38(7), 723-733.

${ }^{177}$ Harris, J.; Bruening, M. Langmuir, 2000, 16, 2006-2013.; Laurent, D.; Schlenoff, J. Langmuir, 1997, 13, 1552-1557.;

${ }^{178}$ Harris, J.; Stair, J.; Bruening, M. Chem Mater., 2000, 12(7), 1941-1946.

${ }^{179}$ Han, S.; Lindholm-Sethson, B. Electrochimica Acta, 1999, 45(6), 845-853.

${ }^{180}$ Oyama, N.; Shimomura, T.; Shigehara, K.; Anson, F. J. Electroanal. Chem. Interf. Electro., 1980, 112(2), 271-280.

181 Pays, M.; Korba, T.; Bos, M.; van der Linden, W. Talanta, 1991, 38(7), 723-733.

182 Ibarz, G.; Dahne, L.; Donath, E.; Mohwald, H. Chem. Mater., 2002, 14(10), 4059-4062.

183 Takita, R.; Okamura, Y.; Endo, Y.; Anzai, J. Electroanal., 2006, 18(16), 1627-1630.

${ }^{184}$ Farhat, T.; Schlenoff, J. J. Am. Chem. Soc., 2003, 125, 4627-4636.

${ }^{185}$ Harris, J.; Stair, J.; Bruening, M. Chem Mater., 2000, 12(7), 1941-1946.

${ }^{186}$ Garcia-Morales, V.; Silva, T.; Moura, C.; Manzanarez, J.; Silva, F. Jo. Electroanal. Chem., 2004, 569, 111-119.; Antipov, A.; Sukhorukov, G. Adv. Coll. Interface Sci., 2004, 111, 49-61.; Krasemann, L.; Tieke, B. Mat. Sci. Eng., 1999, 8-9, 513-518.;

Peyratout, C.; Dahne, L. Angew. Chem. Int. Ed., 2004, 43, 3762-3783.

${ }^{187}$ Hof, M.; Fidler, V. Fluorescence Spectroscopy in Biology, Springer: USA, 2005.

${ }^{188}$ Lichtman, J.; Conchello, J. Nature Methods, 2005, 2(12), 910-919.

${ }^{189}$ Nairn, R. Fluorescent Protein Tracing, Church Livingstone : USA, 1976.

190 Lichtman, J.; Conchello, J. Nature Methods, 2005, 2(12), 910-919.

${ }^{191}$ Van Duuren, B. Chem Rev., 1963, 63, 325-354.

192 Nairn, R. Fluorescent Protein Tracing, Church Livingstone : USA, 1976.

${ }^{193}$ Wolfbeis, O. Fluorescence Spectrsoscopy, Springer-Verlag: USA, 1993.

194 Lakowicz, J..Principles of Fluorescence Anisotropy.Springer: USA, third edition, 2006.

195 Adapted from Horiba:

http://www.horiba.com/fileadmin/uploads/Scientific/Documents/Fluorescence/Anis otropy_FL-3.pdf

${ }^{196}$ Technical Reports "Fluorescence Anisotropy Studies". Available at http://www.horiba.com/fileadmin/uploads/Scientific/Documents/Fluorescence/Anis otropy_FL-3.pdf

${ }^{197}$ Chen, R.; Edelhoch, H. Biochemical Fluorescence Concepts, Marcel Dekker: USA, 1975. 
${ }^{198}$ Smith, B.Infrared Spectral Interpretation: A systematic Approach CRC Press: Canada, 1999.

${ }^{199}$ Keith J.; Laidler, J.; Meiser, H. Physical Chemistry. Benjamin/Cummings Publishing Company, Inc., 1982.

${ }^{200}$ Brigham, E.; Morrow, R. The Fast Fourier Transform. IEEE Spectrum: USA, 1967.

${ }^{201}$ Hilliard, R.; Shepherd, G. Jo. Optical Society of America, 1966, 56(3), 362-369.

${ }^{202}$ Garrett, R.; Grisham, C. Biochemistry. Thomas Books: USA. Second edition., 1999.

${ }^{203}$ Chandrasekaran, S. Plant and Soil, 1969, 299-304.

${ }^{204}$ Osterberg, R.; Szajdak, L. Environment International, 1996, 22(3), 331-334

${ }^{205}$ Reinemann C.; Stoltenburg, R.; Strehlitz, B. Anal Chem., 2009, 81, 3973-3978.

${ }^{206}$ Beaucage, S.; lyer, R. Tetrahedron, 1992, 48(12), 2223-2311.

${ }^{207}$ Mann, D.; Reinemann, C.; Stoltenburg, R.; Strehlitz, B. Biochem. Biophys. Research Comm., 2005, 338, 1928-1934.

${ }^{208}$ Reinemann, C.; Stoltenburg, R.; Strehlitz, B. Anal. Chem., 2009, 81, 3973-3978.

${ }^{209}$ Adapted from: http://www.millipore.com/images/xl/Amicon05_Desalting71\%5B83650-ALL\%5D.jpg

${ }^{210}$ Nah, J.; Jang, M. Jo. Polymer Science, 2002, 40(21), 3796-3803.

211 Luu, K.; Phan, A.; Kuryavyi, V.; Lacroix, L.; Patel, D. J. Am. Chem. Soc. 2006, 128, $9963-$ 9970.

212 Wang, L.; Bloomfield, V. Macromolecules, 1990, 23, 804-809.

${ }^{213}$ Gao, C.; Donath, E.; Moya, S.; Dudnik, V.; Mohwald, H. The European Physical Journal E. 2001, 5, 21-27.

${ }^{214}$ McAloney, R.; Sinyor, M.; Dudnik, V.; Goh, C. Langmuir, 2001, 17(21), 6655-6663.

215 Antipov, A.; Sukhorukov, G.; Mohwald, H. Langmuir, 2003, 19, 2444.

${ }^{216}$ Willis, M. C.; Collins, B.; Zhang, T.; Green, L.; Sebesta, D.; Bell, C.; Kellogg, E.; Gill, S.; Magallanez, A.; Knauer, S.; Bendele, R.; Gill, P.;. Janjic, N. Bioconj. Chem., 1998, 9, $573-$ 582.

${ }^{217}$ Cao, Z.; Tong, R.; Mishr, A.; Xu, W.; Wong, G.; Cheng, J.; Lu, Y. Angew. Chem. Int. Ed.2009, 48, $6494-6498$.

${ }^{218}$ Kang, H.; O'Donoghue, M.; Liu, H.; Tan, W., Chem Comm., 2010, 46, 249-251.

${ }^{219}$ Farokhzad, O.; Cheng, J.; Teply, B.; Sherifi, I.; Jon, S.; Kantoff, P.; Richie, J.; Langer, R. Proc. Natl. Acad. Sci. U. S. A., 2006, 103, 6315-6320.; Gu, F.; Zhang, L.; Teply, B.; Mann, N.; Wang, A.; Radovic- Moreno, A.; Langer, R.; Farokhzad, O. Proc. Natl. Acad. Sci. 
U. S. A., 2008, 105, 2586-2591. ; Dhar, S.; Gu, F.; Langer, R.; Farokhzad, O.; Lippard, S. Proc. Natl. Acad. Sci. U. S. A., 2008, 105, 17356-17361.

${ }^{220}$ Wua, Y.; Sefaha, K.; Liu, H.; Wang, R.; Tan, W. Proc. Natl. Acad. Sci. U. S. A., 2010, 107, 5-10.

221 Miyamoto, D.; Tang, Z.; Takarada, T.; Maeda, M. Chem. Commun., 2007, 4743-4745.

222 Wilson, C.; Szostak, J., 1998, 5, 609-617.

${ }^{223}$ Tanford, C. The Hydrophobic Effect: Formation of Micelles and Biological Membranes. John Wiley \& Sons: USA, 1973.

${ }^{224}$ Discher, D.; Ahmed, F. Annu. Rev. Biomed. Eng., 2006, 8, 323-341.

${ }^{225}$ Discher, D.; Eisenberg, A. Science, 2002, 207, 967-973.

${ }^{226}$ Sugiyama, J.; Glass, D.; Yancopoulos, G.; Hall, Z. The. Jo. Cell. Bio., 1997, 139(1), 181191.

${ }^{227}$ Zhang, C.; Kim, S. Pacific Symposium on Biocomputing, 2000, 5, 350-361.

${ }^{228}$ Germain, R. Curr. Biol., 1997, 7(10), R640-644.

${ }^{229}$ Zhang, C.; Kim, S. Pacific Symposium on Biocomputing, 2000, 5, 350-361.

${ }^{230}$ Bray, D.; Levin, M.; Morton-Firth, C. Letters to Nature, 1998, 393, 85-88.

${ }^{231}$ Ayela, C.; Roquet, F.; Valera, L.; Grainer, C.; Nicu, L.; Pugniere, M. Biosen. Bioelec., 2007, 22(12), 3113-3119.

232 Fylstra, D.; Lasdon, L.; Watson, J.; Waren, A. Reliab. Comput. 1998, 9, 143-159.

${ }^{233}$ Vandermeulen, G.; Klok, H. Macromol. Biosci., 2004, 4, 383-398.

${ }^{234}$ Li, Z.; Zhang, L.; Fullhart, P.; Mirkin, C. NanoLetters, 2004, 4(6), 1055-1058.;

Alemdaroglu, F.; Ding, K.; Berger, R.; Herrmann, A. Angew. Chem. Int. Ed., 2006, 45, 4206-4210.

${ }^{235}$ Sharma, A.; Sharma, U. Inter. Jo. Pharm., 1997, 154(2), 123-140.

${ }^{236}$ O'Neil, C.; Suzuki, T.; Demurtas, D.; Finka, A.; Hubbell, J. Langmuir, 2009, 25(16), 9025-9029.

${ }^{237}$ Metwalli, E.; Moulin, J.; Gebhardt, R.; Cubitt, R.; Tolkach, A.; Kulozik, U.; Buschbaum, P. Langmuir, 2009, 25(7), 4124-4131.

${ }^{238}$ Chattopadhyay, N.; Zastre, J.; Wong, H.; Wu, X.; Bendayan, R. Pharm. Research, 2008, 25(10), 2262-2271.

${ }^{239}$ O'Neil, C.; Suzuki, T.; Demurtas, D.; Finka, A.; Hubbell, J. Langmuir, 2009, 25(16), 9025-9029.

${ }^{240}$ Fanciullino, R.; Ciccolini, J. Current Med. Chem., 2009, 16(33), 4361-4373. 
${ }^{241}$ Cui, Y.; Wu, Z.; Liu, X.; Ni, R.; Zu, X.; Ma, L.; Liu, J. AAPS PharmSciTech, 2010, 11(2), 878-884.

242 Muscariello, L.; Rosso, F.; Marino, G.; Giordano, A.; Barbarisi, M.; Cafiero, G.; Barbarisi, A. Jo. Cell. Phys., 2005, 205, 328-334.

${ }^{243}$ Gagegaard, N. Biotechnic \& Histochemistry, 2006, 81(2-3), 87-97.

${ }^{244}$ Riese, D.; Vos, W.; Wegdam, G.; Poelwijk, F. Phys. Review. E. 2000, 61(2), 1676-1680.

245 Taken from Avanti Lipids.

http://www.avantilipids.com/index.php?option=com_content\&view=article\&id $=532$ \&ltemid $=296$

${ }^{246}$ Wilson, C. and Szostak, J. W. (1998) Isolation of a fluorophore-specific DNA aptamer with weak redox activity, Chem. Biol. 5, 609-617.

${ }^{247}$ Babendure, J.; Adams, S.; Tsien, R. Jo. Am. Chem. Soc., 2003, 125(48), 14716-14717.

${ }^{248}$ Wua, Y.; Sefaha, K.; Liu, H.; Wang, R.; Tan, W. Proc. Natl. Acad. Sci. U. S. A., 2010, $107,5-10$. 